\title{
Geometry and structure of Lipschitz-free spaces and their biduals
}

\author{
Ph.D. dissertation
}

Corrected version

October 2020

\author{
Author: \\ Dr. Ramón José Aliaga Varea \\ Supervisor: \\ Dr. Antonio José Guirao Sánchez
}





\section{Abstract}

Lipschitz-free spaces $\mathcal{F}(M)$ are canonical linearizations of arbitrary complete metric spaces $M$. More specifically, $\mathcal{F}(M)$ is the unique Banach space that contains an isometric copy of $M$ that is linearly dense, and such that any Lipschitz mapping from $M$ into some Banach space $X$ extends to a bounded linear operator from $\mathcal{F}(M)$ into $X$. Those spaces are a very powerful tool for studies of the nonlinear geometry of Banach spaces, as they allow the application of well-known classical linear techniques to nonlinear problems. But this effort is only worthwhile if we have sufficient knowledge about the structure of $\mathcal{F}(M)$. The systematic study of Lipschitz-free spaces is rather recent and so the current understanding of their structure is still quite limited. This thesis is framed within the general program of studying the structure of general Lipschitz-free spaces.

We start our study by developing some basic tools for the general theory of Lipschitz-free spaces. First we introduce weighting operators and use them to solve Weaver's conjecture that all normal functionals in the bidual $\mathcal{F}(M)^{* *}$ are weak* continuous. Next we prove the intersection theorem, which essentially says that the intersection of Lipschitz-free spaces is again a Lipschitz-free space. That result allows us to develop the concept of support of an element of $\mathcal{F}(M)$, analogous to the support of a measure. Furthermore, we extend the use of these tools to the bidual $\mathcal{F}(M)^{* *}$ and apply them to establish a decomposition of the bidual into spaces of functionals that are "concentrated at infinity" and "separated from infinity", respectively.

With these tools at our disposal, we undertake the study of two particular aspects of Lipschitz-free spaces. First we analyze the relationship between $\mathcal{F}(M)$ and spaces of measures on $M$. In particular, we obtain characterizations of those elements of $\mathcal{F}(M)$ that can be represented as integration against a (not necessarily finite) Borel measure on $M$ and vice versa, and we show that their supports agree. We also identify those metric spaces such that every element of $\mathcal{F}(M)$ can be represented by a Borel measure. This analysis is generalized to the bidual $\mathcal{F}(M)^{* *}$, using measures on the uniform compactification of $M$ in that case and obtaining similar results. We also derive some consequences for those elements of $\mathcal{F}(M)$ and 
$\mathcal{F}(M)^{* *}$ that can be expressed as the difference between two positive elements, such as the existence of an analog of the Jordan decomposition for measures.

Secondly, we study the extremal structure of the unit ball of $\mathcal{F}(M)$ and provide some contributions to the general program of finding purely geometric characterizations of all of its extremal elements. Namely, we characterize all of its preserved extreme points, and its extreme and exposed points of finite support. We also give a full description of the extremal structure of the positive unit ball. The theory of supports developed previously plays a crucial role in the proofs of these results. 


\section{Resumen}

Los espacios libres Lipschitz $\mathcal{F}(M)$ son linearizaciones canónicas de espacios métri$\cos M$ cualesquiera. Más concretamente, $\mathcal{F}(M)$ es el único espacio de Banach que contiene una copia isométrica de $M$ que es linearmente densa, y tal que toda aplicación Lipschitz de $M$ en cualquier espacio de Banach $X$ puede extenderse a un operador linear continuo de $\mathcal{F}(M)$ en $X$. Estos espacios suponen una herramienta muy potente para el estudio de la geometría no lineal de espacios de Banach, al permitir la aplicación de las técnicas lineales clásicas, bien conocidas, a problemas no lineales. Pero este esfuerzo sólo merece la pena si se dispone de un conocimiento lo bastante detallado de la estructura de $\mathcal{F}(M)$. El estudio sistemático de los espacios libres Lipschitz es bastante reciente y, por ello, dicho conocimiento es todavía más bien limitado. Esta tesis se enmarca en el programa general de estudio de la estructura espacios libres Lipschitz genéricos.

Empezamos nuestro estudio desarrollando algunas herramientas básicas para la teoría general de espacios libres Lipschitz. Primero definimos operadores de ponderación en espacios Lipschitz y los usamos para demostrar la conjetura de Weaver de que todos los funcionales normales del bidual $\mathcal{F}(M)^{* *}$ son débil* continuos. A continuación demostramos el teorema de la intersección, que en esencia dice que la intersección de espacios libres Lipschitz es de nuevo un espacio libre Lipschitz. Este resultado nos permite desarrollar el concepto de soporte de un elemento de $\mathcal{F}(M)$, análogo al de soporte de una medida. Además, extendemos el uso de estas herramientas al bidual $\mathcal{F}(M)$ y las usamos para establecer una descomposición del bidual en espacios de funcionales que están "concentrados en el infinito" y "separados del infinito", respectivamente.

Con estas herramientas en nuestro poder, emprendemos el estudio de dos aspectos concretos de los espacios libres Lipschitz. En primer lugar analizamos la relación entre $\mathcal{F}(M)$ y los espacios de medidas sobre $M$. En particular, obtenemos caracterizaciones de los elementos de $\mathcal{F}(M)$ que pueden representarse como la integración con respecto a una medida de Borel (no necesariamente finita) sobre $M$ y viceversa, y probamos que el soporte coincide con el de la medida asociada. También identificamos los espacios métricos $M$ en los cuales todo elemento de $\mathcal{F}(M)$ puede 
ser representado como una medida de Borel. Este análisis se generaliza al bidual $\mathcal{F}(M)^{* *}$, utilizando en este caso medidas sobre la compactificación uniforme de $M$ y llegando a resultados similares. Obtenemos también algunas consecuencias para los elementos de $\mathcal{F}(M)$ y $\mathcal{F}(M)^{* *}$ que pueden expresarse como diferencia de dos elementos positivos, como la existencia de un análogo de la descomposición de Jordan para medidas.

En segundo lugar, estudiamos la estructura extremal de la bola unidad de $\mathcal{F}(M)$ y hacemos algunas contribuciones al programa general consistente en encontrar caracterizaciones puramente geométricas de todos sus elementos extremales. Concretamente, caracterizamos los puntos extremos preservados de la bola, así como aquellos puntos extremos y expuestos que tienen soporte finito. Además damos una descripción completa de la estructura extremal de la parte positiva de la bola unidad. La teoría de los soportes en $\mathcal{F}(M)$ desarrollada anteriormente juega un papel crucial en las demostraciones de estos resultados. 


\section{Resum}

Els espais lliures Lipschitz $\mathcal{F}(M)$ són linearitzacions canòniques d'espais mètrics $M$ qualssevol. Més concretament, $\mathcal{F}(M)$ és l'únic espai de Banach que conté una còpia isomètrica de $M$ que és linealment densa, i tal que tota aplicació Lipschitz de $M$ en qualsevol espai de Banach $X$ pot ser estesa a un operador lineal continu de $\mathcal{F}(M)$ en $X$. Aquests espais són una eina molt potent per a l'estudi de la geometria no lineal d'espais de Banach, ja que permeten l'aplicació de les tècniques lineals clàssiques, ben conegudes, a problemes no lineals. Però aquest esforç nomes val la pena si es disposa d'un coneixement bastant detallat de l'estructura de $\mathcal{F}(M)$. L'estudi sistemàtic dels espais lliures Lipschitz és bastant recent i, per això, aquest coneixement és encara prou limitat. Aquesta tesi s'emmarca en el programa general d'estudi de l'estructura dels espais lliures Lipschitz genèrics.

Comencem el nostre estudi desenvolupant algunes eines bàsiques per a la teoria general d'espais lliures Lipschitz. Primer definim operadors de ponderació en espais Lipchitz i els fem servir per demostrar la conjectura de Weaver que tots els funcionals normals del bidual $\mathcal{F}(M)^{* *}$ son feble* continus. A continuació demostrem el teorema de la intersecció, que en essència diu que la intersecció d'espais lliures Lipschitz és de nou un espai lliure Lipschitz. Aquest resultat ens permet desenvolupar el concepte de suport d'un element de $\mathcal{F}(M)$, anàleg al de suport d'una mesura. A més, estenem l'ús d'aquestes eines al bidual $\mathcal{F}(M)^{* *}$ i les fem servir per establir una descomposició del bidual en espais de funcionals que estan "concentrats a l'infinit" i "separats de l'infinit", respectivament.

Amb aquestes eines al nostre abast, emprenem l'estudi de dos aspectes concrets dels espais lliures Lipschitz. En primer lloc, analitzem la relació entre $\mathcal{F}(M)$ i els espais de mesures sobre $M$. En particular, obtenim caracteritzacions dels elements de $\mathcal{F}(M)$ que poden representar-se com la integració respecte a una mesura de Borel (no necessàriament finita) sobre $M$ i viceversa, i provem que el suport coincideix amb el de la mesura associada. També identifiquem els espais mètrics $M$ on tot element de $\mathcal{F}(M)$ pot ser representat com una mesura de Borel. Aquesta análisi es generalitza al bidual $\mathcal{F}(M)^{* *}$, utilitzant en aquest cas mesures sobre la compactificació uniforme de $M$ i arribant a resultats similars. També 
obtenim algunes conseqüències per als elements de $\mathcal{F}(M)$ i $\mathcal{F}(M)^{* *}$ que poden expressar-se com a diferència de dos elements positius, com ara l'existència d'un anàleg de la descomposició de Jordan per a mesures.

En segon lloc, estudiem l'estructura extremal de la bola unitat de $\mathcal{F}(M)$ i fem algunes contribucions al programa general consistent en trobar caracteritzacions purament geomètriques de tots els seus elements extremals. Concretament, caracteritzem els punts extrems preservats de la bola, així com aquells punts extrems i exposats que tenen suport finit. A més fem una descripció completa de l'estructura extremal de la part positiva de la bola unitat. La teoria dels suports en $\mathcal{F}(M)$ desenvolupada anteriorment juga un paper crucial en les demostracions d'aquests resultats. 


\section{Contents}

Abstract $\quad$ iii

Resumen $\quad$ v

Resum vii

$\begin{array}{lll}\text { Contents } & \text { ix }\end{array}$

1 Introduction 1

1.1 Notation . . . . . . . . . . . . . . . . . . . 6

2 Basics of Lipschitz and Lipschitz-free spaces 9

2.1 Lipschitz functions and Lipschitz spaces . . . . . . . . . . . . . . 9

2.2 Lipschitz-free spaces . . . . . . . . . . . . . . . . . . . 16

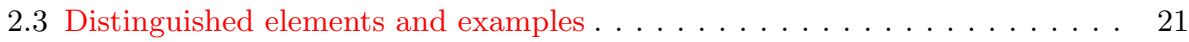

3 Supports in Lipschitz-free spaces and their biduals 29

3.1 Weighting operators $\ldots \ldots \ldots \ldots \ldots \ldots \ldots \ldots \ldots \ldots \ldots$

3.2 Normal functionals $\ldots \ldots \ldots \ldots \ldots \ldots \ldots \ldots \ldots \ldots \ldots \ldots$

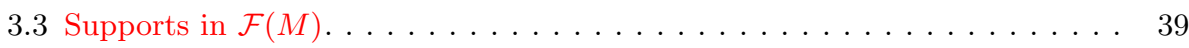

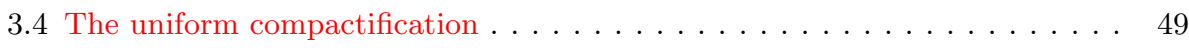

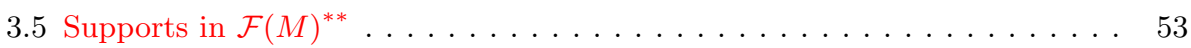

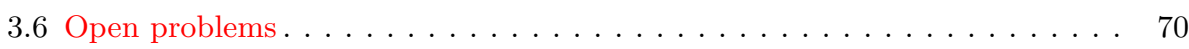


4 Integral representation $\quad 73$

4.1 Radon measures . . . . . . . . . . . . . . . . . . . . 74

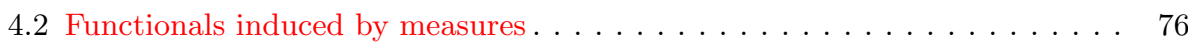

4.3 Majorizable functionals . . . . . . . . . . . . . . . . . . . 89

4.4 Radially discrete spaces . . . . . . . . . . . . . . . . . . . . 100

4.5 Open problems . . . . . . . . . . . . . . . . . . . . 103

5 Extremal structure of the unit ball 105

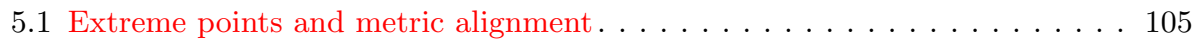

5.2 Extreme and preserved extreme molecules. . . . . . . . . . . . . . 110

5.3 Other extremal elements . . . . . . . . . . . . . . . . . . . 121

5.4 Open problems. . . . . . . . . . . . . . . . . . . . . 127

$\begin{array}{ll}\text { Bibliography } & 131\end{array}$

$\begin{array}{ll}\text { List of symbols } & 135\end{array}$

$\begin{array}{ll}\text { Index of terms } & 137\end{array}$ 


\section{Chapter 1}

\section{Introduction}

Given a complete metric space $(M, d)$ in which an arbitrary point 0 is selected

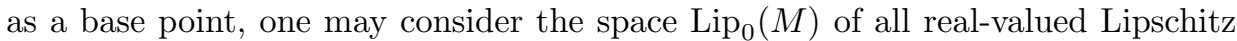
functions on $M$ that vanish at the base point. This turns out to be a Banach space endowed with the norm given by the Lipschitz constant of each function. We may then consider the evaluation functionals $\delta(x) \in \operatorname{Lip}_{0}(M)^{*}$ for each point $x \in M$. The closed space generated by these functionals is called Lipschitz-free space over $M$ and denoted by $\mathcal{F}(M)$. This space has the following fundamental properties:

- It contains an isometric copy $\delta(M)$ of $M$ that is linearly dense.

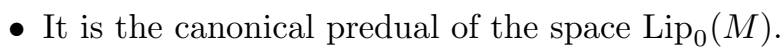

- It satisfies the following universal property: any Lipschitz mapping from $M$ into a Banach space $X$ can be extended to a linear operator from $\mathcal{F}(M)$ into $X$. More generally, any Lipschitz mapping between two metric spaces $M$ and $N$ can be extended to a linear operator between the Lipschitz-free spaces $\mathcal{F}(M)$ and $\mathcal{F}(N)$. Moreover, the norm of the operator is equal to the Lipschitz constant of the original mapping.

The universal property implies that the operation of constructing Lipschitz-free spaces is a functor from the category of metric spaces to the category of Banach spaces. It allows us to transform a complicated (Lipschitz) mapping into a simpler (linear) one at the expense of substituting the domain and range metric spaces by their associated Lipschitz-free spaces, with a more complicated structure. It also allows us, for instance, to dismiss the existence of bi-Lipschitz homeomorphisms between two metric spaces by proving that their Lipschitz-free spaces are not isomorphic to each other. For these reasons, Lipschitz-free spaces are nowadays considered a fundamental tool for the study of the non-linear geometry of Banach 
spaces, as they allow the application of classical linear techniques to non-linear problems.

The construction of the Lipschitz-free spaces can be traced back to the mid- $20^{\text {th }}$ century with the pioneering works of Kantorovich and Rubinstein on optimal transport problems [41], and was rediscovered several times during the subsequent decades by authors such as Arens, de Leeuw, Eells or Johnson, often as an auxiliary tool for a particular problem [10,18,37]. In particular, Kadets [38] and Pestov [46] stated early versions of the universal property (see Theorem 2.2.4). The systematic study of Lipschitz-free spaces begins in the 1990s with the work of Weaver (using the name Arens-Eells spaces). The publication of the first edition of his monograph [52] in 1999, which is still considered the fundamental reference in this field, is a major milestone.

If we restrict ourselves to the more specific topic of Geometry of Banach Spaces, the use of these spaces begins in 2003 with the seminal paper by Godefroy and Kalton [30] where the term Lipschitz-free space is coined, which is now prevalent in this field. That paper focuses on the case where the metric space $M$ under consideration is a Banach space with the norm metric, and it contains the proof of results such as the following:

- $\mathcal{F}\left(\mathbb{R}^{n}\right)$ has the metric approximation property;

- the bounded approximation property of Banach spaces is stable under Lipschitz homeomorphisms;

- if a Banach space contains an isometric copy of another separable Banach space, then it actually contains a linearly isometric copy.

The study of the linear structure (both isometric and isomorphic) of Lipschitz-free spaces began its development in the wake of these results, and it has been growing in interest during the last 15 years.

Currently, Lipschitz-free spaces find widespread use in several branches of mathematics where they are sometimes known by different names, such as Wasserstein-1 spaces in Metric Geometry. ${ }^{1}$ Besides their obvious theoretical interest, one may also find incentives for their study coming from certain branches of applied mathematics. One notable example is Optimal Transport: indeed, the norm of $\mathcal{F}(M)$ may be interpreted as the cost of the optimal solution to a certain transport problem; see e.g. [50]. Hence Lipschitz-free spaces find more or less explicit applications in problems related to PDEs, computer vision, image reconstruction and correction, etc. This is, for instance, one of the motivations behind the paper [45] where the theoretical result is proved that $\mathcal{F}\left(\mathbb{R}^{2}\right)$ is not contained in $\mathcal{F}(\mathbb{R})$. As a practical consequence of this fact, one obtains lower bounds for the execution time of

\footnotetext{
${ }^{1}$ Actually Wasserstein-1 spaces are not exactly the same as Lipschitz-free spaces, but rather a certain linearly generating subset thereof.
} 
certain search algorithms employed in the analysis of 2D images. Let us remark here that the question whether $\mathcal{F}\left(\mathbb{R}^{m}\right)$ can be contained in $\mathcal{F}\left(\mathbb{R}^{n}\right)$ for $m>n>1$, which has analogous interest and applications, is open at the time of writing.

\section{Outline of the thesis}

This thesis is primarily concerned with the isometric structure of Lipschitz-free spaces. More specifically, the starting point of the present research is the study of the extremal structure of the unit ball $B_{\mathcal{F}(M)}$. The roots of this study go back to the 1990s, when Weaver proved that all preserved extreme points of $B_{\mathcal{F}(M)}$ had to be elementary molecules, that is, elements of $\mathcal{F}(M)$ of the form

$$
m_{x y}=\frac{\delta(x)-\delta(y)}{d(x, y)}
$$

for $x \neq y \in M$. Apart from that result, knowledge about this topic was scarce until 2017 when a series of works by García-Lirola, Petitjean, Procházka and Rueda Zoca shed new light on the issue. In [24] they gave a characterization of strongly exposed points of $B_{\mathcal{F}(M)}$ in terms of a geometrical condition on points $x, y$, and in [23] they showed that the sets of preserved extreme points and denting points coincide.

This thesis picks up at this point and continues the program of characterizing the different extremal elements of the unit ball. In particular, some of the results presented here include similar characterizations of preserved extreme points, and of those extreme and exposed points that also have the form $m_{x y}$. We remark here that, at the time of writing, it is still an open problem whether all extreme points are necessarily elementary molecules.

On the way to proving those results, it becomes necessary to establish some fundamental facts about Lipschitz-free spaces that appear to have been previously unknown. The prime example is what we have called the intersection theorem. It roughly says the following: the Lipschitz-free space over the intersection of a family of sets is the same as the intersection of the Lipschitz-free spaces over those sets. That is, "the symbols $\mathcal{F}$ and $\bigcap$ commute". It is perhaps surprising that this elementary-looking fact went unnoticed for so long. However, our proof requires some results about the algebraic structure of Lipschitz spaces, which is often overlooked in favor of the linear structure. The real importance of this result lies in the fact that it allows us to define another fundamental notion: that of support of an element of $\mathcal{F}(M)$, which possesses the basic properties one would intuitively expect from an object called "support" by analogy with, say, continuous functions or measures.

Let us now briefly describe the structure of this document. After this introduction, Chapter 2 presents the basic theory of Lipschitz and Lipschitz-free spaces and some 
of the most important and paradigmatic examples. An effort has been made to write a self-contained text, so the proofs of virtually all results are included. Most proofs are rather elementary and only basic knowledge of Banach Space theory is required.

The rest of the document presents the research conducted during the course of the thesis. The material is not presented in chronological order; instead, we start with the fundamental, general results first and save the applications to the study of extremal structure for last.

In Chapter 3 we extend the basic theory of Lipschitz-free spaces with some general tools. First we briefly develop the theory of weighting operators on $\mathcal{F}(M)$ and, as an elementary (but rather technical) application, we solve the long-standing open problem by Weaver asking whether all normal functionals in $\operatorname{Lip}_{0}(M)^{*}$ belong to $\mathcal{F}(M)$ (see Theorem 3.2.5). Next we move to the main result in the chapter, and possibly the whole thesis: the intersection theorem (see Theorem 3.3.5). We use it to define supports of elements of $\mathcal{F}(M)$ and prove their most basic properties and characterizations. Let us mention just one of them that should be enough to explain our choice of name: the action of an element $m \in \mathcal{F}(M)$ on a Lipschitz function $f$ only depends on the values that $f$ takes on the support of $m$ (see Proposition 3.3.9).

The second half of Chapter 3 is devoted to extending the concept of support to all elements of the bidual $\mathcal{F}(M)^{* *}$. An important change of setting takes place here as it is shown that, in general, it is not possible to give a reasonable definition of such a support as a subset of $M$. Instead, it is necessary to define it as a subset of a compactification of $M$, but the usual approach of considering the Stone-Čech compactification $\beta M$ also leads to inconsistencies. We show that the appropriate choice is the lesser-known uniform compactification $M^{\mathcal{U}}$. We also establish a canonical decomposition of elements of $\mathcal{F}(M)^{* *}$ into a part that is "concentrated at infinity" and a part that is "concentrated away from infinity", and show that in general supports only have meaningful properties for the latter.

Motivated by a question asked by Godefroy at a conference in 2018, we undertake in Chapter 4 a detailed study of the relationship between elements of $\mathcal{F}(M)$ and measures on $M$; note that both can be interpreted as functionals over the space $\operatorname{Lip}_{0}(M)$. In particular, when an element of $\mathcal{F}(M)$ can be identified with such a measure then the concept of support agrees for both representations. As it turns out, a completely analogous study can be carried out for elements of $\mathcal{F}(M)^{* *}$ and measures on the uniform compactification $M^{\mathcal{U}}$. We characterize those measures that correspond to bounded functionals on $\operatorname{Lip}_{0}(M)$ and conversely, those elements of $\mathcal{F}(M)$ and $\mathcal{F}(M)^{* *}$ that admit an integral representation. The most important technical result is that, out of those measures on $M^{\mathcal{U}}$ that represent elements of $\mathcal{F}(M)^{* *}$, the ones that yield elements of $\mathcal{F}(M)$ are precisely those that are concentrated on $M$ (see Theorem 4.2.7). 
After that, we study the majorizable elements of $\mathcal{F}(M)$ and $\mathcal{F}(M)^{* *}$, that is, those that can be expressed as the difference between two positive elements. We show that this family of elements agrees almost exactly with that of elements that admit an integral representation. As a consequence, we are able to prove some unexpected results: for instance, when $M$ is compact and the base point is isolated, any majorizable element of $\mathcal{F}(M)^{* *}$ actually belongs to $\mathcal{F}(M)$ (see Corollary 4.3.10). We are also able to show that majorizable elements of $\mathcal{F}(M)$ admit a canonical minimal representation as a difference of positive elements, analogous to the Jordan decomposition for measures (see Theorem 4.3.15). Finally, we characterize those metric spaces where every element of $\mathcal{F}(M)$ is majorizable or can be represented by a measure.

Finally, in Chapter 5 we apply the previous ideas to the study of the extremal structure of $B_{\mathcal{F}(M)}$. First we provide geometric characterizations of the preserved extreme points and of the extreme points that happen to be elementary molecules. Although these results appeared originally on separate papers $[1,5]$, here we give an almost simultaneous proof of both characterizations (see Theorems 5.2.6 and 5.2.9). We end the chapter by providing some supplementary results concerning other extremal elements, such as exposed molecules and extreme points of the positive unit ball.

Each chapter includes a small final section where some comments and open problems related to its content are collected.

Most of the research contained here has been carried out in collaboration with Eva Pernecká (Czech Technical University in Prague). This includes all results in Chapters 3 and 4 and part of Chapter 5, which appear in the papers [4-6] and in the preprint [3]. Some results in Chapter 5 have been obtained together with Antonio José Guirao (Universitat Politècnica de València) [1] and with Colin Petitjean and Antonín Procházka (Université Bourgogne-Franche-Comté) [6].

Other topics have also been studied with these and other collaborators during the course of the thesis. We summarize some of these unrelated results here:

- In [7], we characterize those metric spaces $M$ such that $\mathcal{F}(M)$ embeds into $\ell_{1}$ isometrically and almost isometrically.

- In [2], we show that certain Banach space properties are compactly determined, in the sense that they are satisfied by $\mathcal{F}(M)$ if and only if they are satisfied by $\mathcal{F}(K)$ for every compact $K \subset M$. In particular this holds for weak sequential completeness, the Schur property and, with a small modification, the approximation property and the Dunford-Pettis property.

- In [8], we characterize points of Gâteaux and Fréchet differentiability of the norm in $\mathcal{F}(M)$ under various assumptions. This is a joint work with Abraham Rueda Zoca (Universidad de Granada). 
We have chosen not to include the details of these results in this document, not even as appendices, as they are only tangentially relevant to the research included here (and to each other). Instead, we have preferred to keep this dissertation focused on a smaller number of selected topics that are more strongly related.

\section{Acknowledgments}

The author would like to thank Marek Cúth, Michal Doucha, Antonio José Guirao, Gilles Lancien and Eva Pernecká for their careful reading and correction of this document or parts of it.

Some activities related to this thesis were partially supported by the Spanish Ministry of Economy, Industry and Competitiveness under Grant MTM2017-83262C2-2-P, and by a travel grant of the Institute of Mathematics (IEMath-GR) of the University of Granada. Part of this research was conducted during visits to the Czech Technical University in Prague in 2018 and 2020, the Laboratoire de Mathématiques de Besançon in 2019, and the University of Granada in 2020. The author wishes to express his gratitude for the hospitality and the excellent working conditions during his visits.

\section{$1.1 \quad$ Notation}

Basic knowledge of Banach space theory is assumed throughout the text, including the usual cornerstone theorems of Functional Analysis and the fundamentals of weak and weak ${ }^{*}$ topologies. The comprehensive book [22] is recommended as a reference; the material contained in the first three chapters therein is sufficient. Some elementary knowledge of topology and measure theory is also required. Most of the necessary information can be found e.g. in chapters 1, 2 and 6 of [47]; for basic background on nets in topology, see e.g. [44, §29]. No previous knowledge about Lipschitz or Lipschitz-free spaces is assumed, but we recommend [53] as a reference and for a wealth of further information about these objects.

Let us briefly describe the most common notation we will be using throughout the thesis. The notation that is more specific to the subject will be described as it is introduced in the text, and is collected in the list of symbols at the end of this document.

Unless specified otherwise, $M$ will always denote a complete metric space with metric $d$. We will moreover assume that $M$ is pointed, that is, a certain point of $M$ will be selected as a base point and denoted by 0 . The open ball of radius $r$ 
around $p \in M$ will be denoted $B(p, r)$. We will also use the notation

$$
\begin{aligned}
d(x, A) & =\inf \{d(x, a): a \in A\} \\
d(A, B) & =\inf \{d(a, b): a \in A, b \in B\} \\
\operatorname{rad}(A) & =\sup \{d(a, 0): a \in A\} \\
\operatorname{diam}(A) & =\sup \{d(a, b): a, b \in A\}
\end{aligned}
$$

for $x \in M$ and $A, B \subset M$; the last two quantities will be called the radius and diameter of $A$, respectively. The characteristic function of $A$ will be denoted by $\chi_{A}$ (i.e. $\chi_{A}(x)=1$ for $x \in A$ and $\chi_{A}(x)=0$ for $x \notin A$ ).

We will deal exclusively with real Banach spaces and real scalars. The closed unit ball and unit sphere of a Banach space $X$ will be denoted by $B_{X}$ and $S_{X}$, respectively. The dual of $X$ will be denoted by $X^{*}$, and the dual action of $x^{*} \in X^{*}$ on $x \in X$ as $\left\langle x, x^{*}\right\rangle=x^{*}(x)$. The annihilator of a subset $A \subset X$ will be written as $A^{\perp}$, and the preannihilator of a subset $A \subset X^{*}$ as $A_{\perp}$.

The space of continuous linear operators from $X$ into another Banach space $Y$ shall be written as $\mathcal{L}(X, Y)$. If $X$ and $Y$ are linearly isometric, we will write $X \cong Y$; if they are linearly isomorphic, we will write $X \sim Y$ instead. The standard unit vectors of a $c_{0}(\Gamma)$ or $\ell_{p}(\Gamma)$ space $(1 \leq p \leq \infty)$ will be denoted $e_{\gamma}$, for $\gamma \in \Gamma$. We will write $\operatorname{span}(A)$ for the linear span of $A \subset X$, i.e. the set of finite linear combinations of elements of $A$, and $\operatorname{conv}(A)$ for its convex hull, i.e. the set of finite convex combinations. We will use the notation $\overline{\operatorname{span}}(A)$ and $\overline{\operatorname{conv}}(A)$ for their respective closures. The set of extreme points of $A$ will be $\operatorname{denoted}$ by $\operatorname{ext}(A)$.

We will write $x_{i} \rightarrow x$ to state that a net $\left(x_{i}\right)$ converges to $x$. Convergence shall be understood to hold with respect to the metric or norm topology when dealing with metric or Banach spaces, respectively. When convergence with respect to a specific topology $\tau$ is meant, we will write $x_{i} \stackrel{\tau}{\longrightarrow} x$ instead. The symbols $\tau=w, w^{*}$ will be used for the weak and weak* topology, respectively. When dealing with a function space, $\tau=p$ will be used for the topology of pointwise convergence.

Finally, if $f$ and $g$ are elements of some space of real-valued functions, their pointwise maximum and minimum will be denoted by $f \vee g=\max \{f, g\}$ and $f \wedge g=\min \{f, g\}$. More generally, the pointwise supremum and infimum of a collection $\left(f_{i}\right)$ of real-valued functions will be denoted by $\bigvee_{i} f_{i}=\sup _{i} f_{i}$ and $\bigwedge_{i} f_{i}=\inf _{i} f_{i}$, respectively. We shall also write $f \leq g$ when $f$ is less or equal than $g$ pointwise, and denote $f^{+}=f \vee 0$ and $f^{-}=(-f) \vee 0$. The space of continuous real-valued functions on a topological space $X$ will be denoted by $C(X)$. 



\section{Chapter 2}

\section{Basics of Lipschitz and Lipschitz-free spaces}

In this chapter, Lipschitz functions and Lipschitz-free spaces will be defined and their basic properties will be described. With few exceptions, the proofs of all results have been included since they are elementary. After that, some of the most important elements in Lipschitz-free spaces will be introduced (molecules, and positive and majorizable elements), which will play a large role in Chapters 4 and 5. Finally, we will present some paradigmatic examples of Lipschitz and Lipschitz-free spaces over familiar metric spaces.

\subsection{Lipschitz functions and Lipschitz spaces}

The following definition is well known but central to this thesis:

Definition 2.1.1. Let $f: M \rightarrow N$ be a mapping between two metric spaces $\left(M, d_{M}\right)$ and $\left(N, d_{N}\right)$. The Lipschitz constant of $f$ is defined as the value

$$
\|f\|_{L}=\sup \left\{\frac{d_{N}(f(x), f(y))}{d_{M}(x, y)}: x \neq y \in M\right\} .
$$

The function $f$ is said to be Lipschitz if $\|f\|_{L}<\infty$.

Mappings with a Lipschitz constant less or equal to 1, i.e. such that

$$
d_{N}(f(x), f(y)) \leq d_{M}(x, y)
$$

for any $x, y \in M$, are also called non-expansive. In particular, if $f$ is an isometry i.e. such that $d_{N}(f(x), f(y))=d_{M}(x, y)$ for any $x, y \in M$, then $\|f\|_{L}=1$. 
An important basic fact is that the composition of Lipschitz mappings is again Lipschitz:

Proposition 2.1.2. Let $M, N, P$ be metric spaces and consider mappings $f$ : $M \rightarrow N$ and $g: N \rightarrow P$. Then $\|g \circ f\|_{L} \leq\|g\|_{L} \cdot\|f\|_{L}$. If $g$ is an isometry then $\|g \circ f\|_{L}=\|f\|_{L}$.

Proof. Let $x \neq y \in M$. If $f(x)=f(y)$ then $(g \circ f)(x)=(g \circ f)(y)$, otherwise

$$
\frac{d_{P}((g \circ f)(x),(g \circ f)(y))}{d_{M}(x, y)}=\frac{d_{P}(g(f(x)), g(f(y)))}{d_{N}(f(x), f(y))} \cdot \frac{d_{N}(f(x), f(y))}{d_{M}(x, y)} \leq\|g\|_{L}\|f\|_{L}
$$

and taking suprema we get the desired inequality. If $g$ is an isometry then the left fraction in the product above is equal to 1 whenever $f(x) \neq f(y)$, and so taking suprema yields exactly $\|g \circ f\|_{L}=\|f\|_{L}$.

We will focus on the case where the target space is just the real numbers, so that the Lipschitz constant is given by

$$
\|f\|_{L}=\sup \left\{\frac{|f(x)-f(y)|}{d(x, y)}: x \neq y \in M\right\} .
$$

Notice that absolute values may be omitted in the numerator above because $x$ and $y$ may be interchanged. In a slight abuse of notation, we will refer to fractions of the form $(f(x)-f(y)) / d(x, y)$ as incremental quotients. We shall denote the collection of all real-valued Lipschitz functions on $M$ as $\operatorname{Lip}(M)$. It should be mentioned here that we may assume the metric space $M$ to be complete without loss of generality, as one may obviously identify $\operatorname{Lip}(M)$ with $\operatorname{Lip}(\widehat{M})$ where $\widehat{M}$ is the completion of $M$.

The next proposition describes the behavior of the Lipschitz constant with respect to the most common operations on real-valued functions: sums, products, and taking pointwise minima and maxima. We will use the notation $f \vee g=\max \{f, g\}$ and $f \wedge g=\min \{f, g\}$ for the latter, and also more generally for pointwise suprema and infima of infinite collections of functions.

Proposition 2.1.3. Let $f$ and $g$ be real-valued functions on $M$, and $\lambda \in \mathbb{R}$. Then:

(a) $\|f+g\|_{L} \leq\|f\|_{L}+\|g\|_{L}$,

(b) $\|\lambda f\|_{L}=|\lambda| \cdot\|f\|_{L}$,

(c) $\|f \vee g\|_{L} \leq \max \left\{\|f\|_{L},\|g\|_{L}\right\}$ and $\|f \wedge g\|_{L} \leq \max \left\{\|f\|_{L},\|g\|_{L}\right\}$,

(d) $\|f g\|_{L} \leq\|f\|_{L}\|g\|_{\infty}+\|g\|_{L}\|f\|_{\infty}$. 
Proof. Choose an arbitrary pair of different points $x, y \in M$, and let us estimate the various Lipschitz constants in the statement. First, we have

$$
\frac{|(f+g)(x)-(f+g)(y)|}{d(x, y)} \leq \frac{|f(x)-f(y)|}{d(x, y)}+\frac{|g(x)-g(y)|}{d(x, y)} \leq\|f\|_{L}+\|g\|_{L}
$$

and taking the supremum over all possible pairs $(x, y)$ we get (a). We also have

$$
\frac{|(\lambda f)(x)-(\lambda f)(y)|}{d(x, y)}=|\lambda| \frac{|f(x)-f(y)|}{d(x, y)}
$$

and taking suprema on both sides yields (b). To prove (c), let $h=f \vee g$ and suppose $h(x) \geq h(y)$ without loss of generality. If $h(x)=f(x)$ then

$$
\frac{h(x)-h(y)}{d(x, y)}=\frac{f(x)-h(y)}{d(x, y)} \leq \frac{f(x)-f(y)}{d(x, y)} \leq\|f\|_{L}
$$

and if $h(x)=g(x)$ then we have the same with $g$ in place of $f$. In any case, taking suprema yields $\|h\|_{L} \leq \max \left\{\|f\|_{L},\|g\|_{L}\right\}$. The corresponding inequality for $h=f \wedge g=-((-f) \vee(-g))$ follows immediately. Finally, for statement (d), compute

$$
\begin{aligned}
\frac{|(f g)(x)-(f g)(y)|}{d(x, y)} & \leq \frac{|f(x) g(x)-f(x) g(y)|}{d(x, y)}+\frac{|f(x) g(y)-f(y) g(y)|}{d(x, y)} \\
& =|f(x)| \frac{|g(x)-g(y)|}{d(x, y)}+|g(y)| \frac{|f(x)-f(y)|}{d(x, y)} \\
& \leq\|f\|_{\infty}\|g\|_{L}+\|g\|_{\infty}\|f\|_{L}
\end{aligned}
$$

to obtain the conclusion.

The following shows that the Lipschitz constant also behaves well with respect to pointwise limits.

\section{Proposition 2.1.4.}

(a) If $\left(f_{i}\right)$ is a net of functions $M \rightarrow \mathbb{R}$ that converges pointwise to $f$ then $\|f\|_{L} \leq \liminf _{i}\left\|f_{i}\right\|_{L}$.

(b) If $\left(f_{n}\right)$ is a sequence of functions $M \rightarrow \mathbb{R}$ and $\sum_{n} f_{i}$ converges pointwise, then $\left\|\sum_{n} f\right\|_{L} \leq \sum_{n}\left\|f_{n}\right\|_{L}$.

(c) If $\left\{f_{i}: i \in I\right\}$ is a family of functions $M \rightarrow \mathbb{R}$ that is pointwise bounded above, then $\left\|\bigvee_{i \in I} f_{i}\right\|_{L} \leq \sup _{i \in I}\left\|f_{i}\right\|_{L}$.

(d) If $\left\{f_{i}: i \in I\right\}$ is a family of functions $M \rightarrow \mathbb{R}$ that is pointwise bounded below, then $\left\|\wedge_{i \in I} f_{i}\right\|_{L} \leq \sup _{i \in I}\left\|f_{i}\right\|_{L}$. 
Proof. (a) For any choice of $x \neq y \in M$ we have

$$
\frac{f(x)-f(y)}{d(x, y)}=\lim _{i} \frac{f_{i}(x)-f_{i}(y)}{d(x, y)} \leq \liminf _{i}\left\|f_{i}\right\|_{L} .
$$

(b) Apply (a) to the sequence $\left(g_{n}\right)$ where $g_{n}=\sum_{k=1}^{n} f_{k}$, which satisfies $\left\|g_{n}\right\|_{L} \leq$ $\sum_{k=1}^{n}\left\|f_{k}\right\|_{L}$ by Proposition 2.1.3(a).

(c) Let $\mathfrak{F}$ be the set of all finite subsets of $I$, directed by inclusion, and consider the net $\left(g_{F}\right)_{F \in \mathfrak{F}}$ where $g_{F}=\bigvee_{i \in F} f_{i}$ for $F \in \mathfrak{F}$. It is straightforward to check that $\left(g_{F}\right)$ converges pointwise to $g=\bigvee_{i \in I} f_{i}$, and we have

$$
\left\|g_{F}\right\|_{L} \leq \max _{i \in F}\left\|f_{i}\right\|_{L} \leq \sup _{i \in I}\left\|f_{i}\right\|_{L}
$$

for any $F \in \mathfrak{F}$ by Proposition 2.1.3(d). Applying (a) now yields the conclusion.

(d) is proved in the same way as (c).

Proposition 2.1.3(a,b) shows that $\operatorname{Lip}(M)$ is a vector space and that the Lipschitz constant is a seminorm on $\operatorname{Lip}(M)$. It is obvious that $\|f\|_{L}=0$ if and only if $f$ is a constant function on $M$, hence the Lipschitz constant cannot be a norm on $\operatorname{Lip}(M)$ (unless $M$ is trivial). However, it would be desirable to endow $\operatorname{Lip}(M)$ with a normed space structure in order to be able to use functional analytic methods for the study of Lipschitz functions. There are several possible ways to circumvent this restriction. The most usual one is to consider pointed metric spaces and restrict our attention to the following subspace:

Definition 2.1.5. The Lipschitz space over $M$ is the vector space

$$
\operatorname{Lip}_{0}(M)=\{f \in \operatorname{Lip}(M): f(0)=0\} .
$$

$\operatorname{Lip}_{0}(M)$ is clearly a one-codimensional vector subspace of $\operatorname{Lip}(M)$ and 0 is the only constant function contained in it, so it follows from the previous discussion that $\|\cdot\|_{L}$ is a norm on $\operatorname{Lip}_{0}(M)$. We can actually say more:

Proposition 2.1.6. $\operatorname{Lip}_{0}(M)$ is a Banach space endowed with the norm $\|\cdot\|_{L}$. Moreover, different choices of the base point of $M$ produce linearly isometric Banach spaces.

Proof. It is a well known fact that a normed vector space is complete if and only if every absolutely convergent series is convergent. So let $\left(f_{n}\right)$ be a sequence in $\operatorname{Lip}_{0}(M)$ such that $\sum_{n}\left\|f_{n}\right\|_{L}<\infty$. For every $x \in M$ we have

$$
\left|f_{n}(x)\right|=\left|f_{n}(x)-f_{n}(0)\right| \leq\left\|f_{n}\right\|_{L} d(x, 0)
$$


thus $\sum_{n}\left|f_{n}(x)\right|$ is absolutely convergent, and so the series $\sum_{n} f_{n}$ converges pointwise to a function $f$. It is clear that $f(0)=0$. By Proposition 2.1.4(b), $f \in$ $\operatorname{Lip}_{0}(M)$ with $\|f\|_{L} \leq \sum_{n}\left\|f_{n}\right\|_{L}$. Moreover, we have

$$
\left\|f-\sum_{k=1}^{n} f_{k}\right\|_{L}=\left\|\sum_{k>n} f_{k}\right\|_{L} \leq \sum_{k>n}\left\|f_{k}\right\|_{L}
$$

where the inequality follows from Proposition 2.1.4(b). Thus $\sum_{n} f_{n}$ converges to $f$ in norm, and the first statement is proved.

Now let $e \in M$ and denote by $\operatorname{Lip}_{e}(M)$ the space of Lipschitz functions on $M$ such that $f(e)=0$. Define $T: \operatorname{Lip}_{0}(M) \rightarrow \operatorname{Lip}_{e}(M)$ by $(T f)(x)=f(x)-f(e)$ for $x \in M$. The map $T$ is obviously linear, and $T f-f$ is a constant function for every $f \in \operatorname{Lip}_{0}(M)$ so $\|T f\|_{L}=\|f\|_{L}$. Moreover, if $g \in \operatorname{Lip}_{e}(M)$ then $f=g-g(0) \in$ $\operatorname{Lip}_{0}(M)$ and $T f=g$, thus $T$ is onto. This ends the proof.

The most important function in $\operatorname{Lip}_{0}(M)$, which we shall denote by $\rho$, is given by

$$
\rho(x)=d(x, 0)
$$

for $x \in M$. The triangle inequality implies that $\rho(x)-\rho(y) \leq d(x, y)$ for any $x, y \in M$, hence $\|\rho\|_{L}=1$. More precisely, $\rho$ is the largest element of $B_{\operatorname{Lip}_{0}(M)}$. Indeed, for any $f \in \operatorname{Lip}_{0}(M)$ and $x \in M$ we have $|f(x)| \leq\|f\|_{L} \cdot \rho(x)$, a fact that has already been used in the preceding proof.

Lipschitz spaces, being spaces of real-valued continuous functions, can be expected not to merely possess a natural linear structure but also an algebraic and a latticetheoretic one. Let us briefly comment on those properties.

Proposition 2.1.3(c) shows that $\operatorname{Lip}(M)$ is a Riesz space, i.e. a vector lattice, with the partial order given by the pointwise order. The same can be said about $\operatorname{Lip}_{0}(M)$, as it is closed under the operations $\vee$ and $\wedge$. Moreover, by Proposition 2.1.4 (c,d) the unit ball $B_{\operatorname{Lip}_{0}(M)}$ is a complete lattice, i.e. every subset has a supremum and an infimum. However, $\operatorname{Lip}_{0}(M)$ is not in general a Banach lattice, because $|f| \leq|g|$ does not imply in general that $\|f\|_{L} \leq\|g\|_{L}$ : a simple counterexample is given by $g(x)=x$ and $f(x)=x \sin x$ in $\operatorname{Lip}_{0}[0,2 \pi]$. For the exact conditions when $\operatorname{Lip}_{0}(M)$ is a Banach lattice see [53, p. 187].

Proposition 2.1.3(d) shows that the product of bounded Lipschitz functions is Lipschitz. If $M$ is bounded then any $f \in \operatorname{Lip}(M)$ is bounded, since

$$
|f(x)| \leq\|f\|_{L} \rho(x) \leq \operatorname{rad}(M)\|f\|_{L}
$$

for every $x \in M$, hence $\|f\|_{\infty} \leq \operatorname{rad}(M)\|f\|_{L}$. Thus, in that case $\operatorname{Lip}(M)$ and $\operatorname{Lip}_{0}(M)$ are algebras under the pointwise product, and moreover

$$
\|f g\|_{L} \leq\|f\|_{L} \operatorname{rad}(M)\|g\|_{L}+\|g\|_{L} \operatorname{rad}(M)\|f\|_{L}=2 \operatorname{rad}(M)\|f\|_{L}\|g\|_{L}
$$


by Proposition 2.1.3(d). So $\operatorname{Lip}_{0}(M)$ is not a Banach algebra in general (unless $\left.\operatorname{rad}(M) \leq \frac{1}{2}\right)$, but it can be turned into one by scaling its norm by a constant factor. When $M$ is unbounded, however, $\operatorname{Lip}_{0}(M)$ is not even closed under pointwise products: just notice that

$$
\frac{\rho(x)^{2}-\rho(0)^{2}}{d(x, 0)}=d(x, 0)
$$

is not bounded, so $\left\|\rho^{2}\right\|_{L}=\infty$ and $\rho^{2} \notin \operatorname{Lip}_{0}(M)$.

Next, let us discuss an extension result for Lipschitz functions defined on subsets of $M$. It is an extremely important fact, first proved by McShane [43], that any such function can be extended to the whole space $M$ without increasing its Lipschitz constant:

Theorem 2.1.7 (McShane). Let $N \subset M$ and $f \in \operatorname{Lip}(N)$. Then there is $F \in$ $\operatorname{Lip}(M)$ such that $F \uparrow_{N}=f,\|F\|_{L}=\|f\|_{L}, \sup _{x \in M} F(x)=\sup _{x \in N} f(x)$ and $\inf _{x \in M} F(x)=\inf _{x \in N} f(x)$.

We will, in fact, need some details about the nature of such an extension $F$. The following definition describes the two canonical constructions of $F$. We will show that they are the largest and smallest possible extensions of $f$ to $M$, respectively.

Definition 2.1.8. Let $N \subset M$ and $f \in \operatorname{Lip}(N)$. Then the functions $E^{+} f, E^{-} f$ : $M \rightarrow \mathbb{R}$, defined by

$$
\begin{aligned}
& \left(E^{+} f\right)(x)=\inf \left\{f(y)+\|f\|_{L} d(x, y): y \in N\right\} \\
& \left(E^{-} f\right)(x)=\sup \left\{f(y)-\|f\|_{L} d(x, y): y \in N\right\}
\end{aligned}
$$

for $x \in M$, are called the inf-convolution and sup-convolution of $f$, respectively.

Proposition 2.1.9. The functions $E^{+} f, E^{-} f$ have the following properties:

(i) $\left(E^{+} f\right) \uparrow_{N}=\left(E^{-} f\right) \Gamma_{N}=f$,

(ii) $\left\|E^{+} f\right\|_{L}=\left\|E^{-} f\right\|_{L}=\|f\|_{L}$,

(iii) if $g \in \operatorname{Lip}(M)$ is any function such that $g \uparrow_{N}=f$ and $\|g\|_{L}=\|f\|_{L}$, then $E^{-} f \leq g \leq E^{+} f$.

Proof. Let $x \in N$, then we have $f(x) \leq f(y)+\|f\|_{L} d(x, y)$ for any $y \in N$ and therefore $f(x) \leq\left(E^{+} f\right)(x)$. But we also have, taking $y=x$, that $\left(E^{+} f\right)(x) \leq$ $f(x)+\|f\|_{L} d(x, x)=f(x)$. Hence $\left(E^{+} f\right)(x)=f(x)$. The proof for $E^{-} f$ is similar. This establishes (i).

To prove (ii), let $x_{1}, x_{2} \in M$ and $\varepsilon>0$. By construction there is $y \in N$ such that $f(y)+\|f\|_{L} d\left(x_{2}, y\right)<\left(E^{+} f\right)\left(x_{2}\right)+\varepsilon$, and we have $\left(E^{+} f\right)\left(x_{1}\right) \leq f(y)+$ 
$\|f\|_{L} d\left(x_{1}, y\right)$, therefore

$$
\begin{aligned}
\left(E^{+} f\right)\left(x_{1}\right)-\left(E^{+} f\right)\left(x_{2}\right) & <f(y)+\|f\|_{L} d\left(x_{1}, y\right)-\left(f(y)+\|f\|_{L} d\left(x_{2}, y\right)-\varepsilon\right) \\
& =\|f\|_{L}\left(d\left(x_{1}, y\right)-d\left(x_{2}, y\right)\right)+\varepsilon \\
& \leq\|f\|_{L} d\left(x_{1}, x_{2}\right)+\varepsilon
\end{aligned}
$$

Taking $\varepsilon \rightarrow 0$ and the supremum over pairs $x_{1}, x_{2}$ we get $\left\|E^{+} f\right\|_{L} \leq\|f\|_{L}$. But (i) clearly implies the opposite inequality, therefore $\left\|E^{+} f\right\|_{L}=\|f\|_{L}$. Again, the argument for $E^{-} f$ is similar.

Finally, if $g$ is as in (iii), then for any $x \in M$ and $y \in N$ we have $|g(x)-g(y)| \leq$ $\|g\|_{L} d(x, y)$ i.e.

$$
f(y)-\|f\|_{L} d(x, y) \leq g(x) \leq f(y)+\|f\|_{L} d(x, y) .
$$

Taking the supremum on the left hand side and the infimum on the right hand side yields $\left(E^{-} f\right)(x) \leq g(x) \leq\left(E^{+} f\right)(x)$.

Proof of Theorem 2.1.7. Let $a=\inf _{x \in N} f(x)$ and $b=\sup _{x \in N} f(x)$. Take $g=$ $E^{+} f\left(\right.$ or $\left.E^{-} f\right)$, and let $F=(g \wedge b) \vee a$. By Propositions 2.1.3(c) and 2.1.9 we have $\|F\|_{L} \leq\|g\|_{L}=\|f\|_{L}$, and since $F \uparrow_{N}=f$ we must have $\|F\|_{L}=\|f\|_{L}$. It is also clear that $\sup _{x \in M} F(x) \leq b$ by construction and $\sup _{x \in M} F(x) \geq b$ by restriction to $N$, therefore $\sup _{x \in M} F(x)=b$. Similarly $\inf _{x \in M} F(x)=a$.

Let us provide an alternative interpretation of Theorem 2.1.7:

Corollary 2.1.10. If $N \subset M$, then

$$
B_{\operatorname{Lip}_{0}(N)}=\left\{f \uparrow_{N}: f \in B_{\operatorname{Lip}_{0}(M)}\right\} .
$$

Indeed, it is clear that $\left\|f \uparrow_{N}\right\|_{L} \leq\|f\|_{L}$ as the supremum defining the Lipschitz constant is taken over a smaller set of pairs of points in the left hand side. This yields inclusion $\supset$. The reverse inclusion is precisely McShane's theorem.

To end this section, we mention another property of Lipschitz functions that will be required occasionally. In order to state it, let us first recall the following concept. Given a set $X$, a partition of unity on $X$ is a family $\left\{f_{i}: i \in I\right\}$ of non-negative functions on $X$ such that, for every $x \in X$, only finitely many of the functions $f_{i}$ have a nonzero value on $x$ and moreover $\sum_{i \in I} f_{i}(x)=1$. If $X$ is a topological space and $\left\{U_{i}: i \in I\right\}$ is an open cover of $X$, then the partition of unity $\left\{f_{i}: i \in I\right\}$ is said to be subordinated to $\left\{U_{i}\right\}$ if $\operatorname{supp}\left(f_{i}\right) \subset U_{i}$ for every $i \in I$.

Theorem 2.1.11. For every open cover of $M$ there is a partition of unity subordinated to it that consists of Lipschitz functions.

The proof of this fact is elementary but rather long and technical, so we choose not to include it here; it can be found e.g. in [14, Theorem 2.6.5]. 


\section{$2.2 \quad$ Lipschitz-free spaces}

In the previous section, we have seen that the Lipschitz $\operatorname{space}_{\operatorname{Lip}}(M)$ is a Banach space. We will now prove the less obvious fact that it is actually a dual Banach space.

There are several equivalent ways to construct the predual of $\operatorname{Lip}_{0}(M)$. The most straightforward one is as the linear space generated by the evaluation functionals $\delta(x) \in \operatorname{Lip}_{0}(M)^{*}$, given by

$$
\langle f, \delta(x)\rangle=f(x)
$$

for $f \in \operatorname{Lip}_{0}(M)$ and $x \in M$. In consistency with this notation, we may more generally consider the mapping

$$
\delta: M \rightarrow \operatorname{Lip}_{0}(M)^{*}
$$

that takes each $x \in M$ to its associated evaluation functional $\delta(x)$.

Let us remark that $\delta(0)$ is just the 0 element of $\operatorname{Lip}_{0}(M)^{*}$ because every $f \in$ $\operatorname{Lip}_{0}(M)$ vanishes at the base point. Notice also that the evaluation functionals are linearly independent. Indeed, given any finite set $E \subset M \backslash\{0\}$ and any $x \in E$, the function that takes $x$ to 1 and all other points of $E$ to 0 is Lipschitz on $E$ (any function on a finite metric space is clearly Lipschitz). By McShane's theorem, it can be extended to $f \in \operatorname{Lip}_{0}(M)$ such that $\langle f, \delta(x)\rangle=1$ and $\langle f, \delta(y)\rangle=0$ for $y \in E, y \neq x$. Hence $\delta(E)$ is a linearly independent set.

Proposition 2.2.1. $\delta$ is an isometric embedding of $M$ into $\operatorname{Lip}_{0}(M)^{*}$ that is continuous with respect to the weak* topology of $\operatorname{Lip}_{0}(M)^{*}$.

Proof. Let $x \neq y \in M$. Then

$$
\begin{aligned}
\|\delta(x)-\delta(y)\| & =\sup \left\{\langle f, \delta(x)-\delta(y)\rangle: f \in B_{\operatorname{Lip}_{0}(M)}\right\} \\
& =\sup \left\{f(x)-f(y): f \in B_{\operatorname{Lip}_{0}(M)}\right\} .
\end{aligned}
$$

On one hand, $f(x)-f(y) \leq d(x, y)$ for any $f \in B_{\operatorname{Lip}_{0}(M)}$. On the other hand, the function $f: z \mapsto d(z, y)-d(0, y)$ belongs to $B_{\operatorname{Lip}_{0}(M)}$ and $f(x)-f(y)=d(x, y)$. Thus the supremum in the expression above is exactly $d(x, y)$, and $\delta$ is indeed an isometry. It is also clear that if $x_{i} \rightarrow x$ in $M$ then

$$
\left\langle f, \delta\left(x_{i}\right)\right\rangle=f\left(x_{i}\right) \rightarrow f(x)=\langle f, \delta(x)\rangle
$$

for any $f \in \operatorname{Lip}_{0}(M)$ because such an $f$ is continuous, thus $\delta\left(x_{i}\right) \stackrel{w^{*}}{\longrightarrow} \delta(x)$. That is, $\delta$ is a weak* continuous mapping.

We can now finally define the main object of study in this thesis. 
Definition 2.2.2. The Lipschitz-free space over $M$ is the subspace of $\operatorname{Lip}_{0}(M)^{*}$ given by

$$
\mathcal{F}(M)=\overline{\operatorname{span}} \delta(M)=\overline{\operatorname{span}}\{\delta(x): x \in M\}
$$

The norm in $\mathcal{F}(M)$ will be simply the restriction of the norm in $\operatorname{Lip}_{0}(M)^{*}$.

Theorem 2.2.3. $\mathcal{F}(M)^{*}$ is linearly isometric to $\operatorname{Lip}_{0}(M)$ under the mapping $\Lambda \mapsto \Lambda \circ \delta$, and the corresponding weak* topology on $\operatorname{Lip}_{0}(M)$ coincides on bounded sets with the topology of pointwise convergence.

Proof. For any $\Lambda \in \mathcal{F}(M)^{*}$ define $T(\Lambda)=\Lambda \circ \delta: M \rightarrow \mathbb{R}$. Notice first that for any $x, y \in M$ we have by Proposition 2.2.1

$$
|T(\Lambda)(x)-T(\Lambda)(y)|=|\Lambda(\delta(x)-\delta(y))| \leq\|\Lambda\| d(x, y)
$$

so $\|T(\Lambda)\|_{L} \leq\|\Lambda\|$. In fact, $T(\Lambda) \in \operatorname{Lip}_{0}(M)$ since $\delta(0)=0$. Now, for every $x \in M$, we have

$$
\langle T(\Lambda), \delta(x)\rangle=T(\Lambda)(x)=(\Lambda \circ \delta)(x)=\langle\delta(x), \Lambda\rangle
$$

and, by linear density of the $\delta(x)$, it follows that $\langle T(\Lambda), m\rangle=\langle m, \Lambda\rangle$ for any $m \in \mathcal{F}(M)$. Therefore

$$
\begin{aligned}
\|\Lambda\|=\sup _{m \in B_{\mathcal{F}(M)}}\langle m, \Lambda\rangle & =\sup _{m \in B_{\mathcal{F}(M)}}\langle T(\Lambda), m\rangle \\
& \leq \sup _{\phi \in B_{\mathrm{Lip}_{0}(M)^{*}}}\langle T(\Lambda), \phi\rangle=\|T(\Lambda)\|_{L} .
\end{aligned}
$$

So we conclude $\|\Lambda\|=\|T(\Lambda)\|_{L}$, and $T: \mathcal{F}(M)^{*} \rightarrow \operatorname{Lip}_{0}(M)$ is a linear isometric embedding.

To see that $T$ is onto, choose $g \in \operatorname{Lip}_{0}(M)$ and let $\Lambda \in \mathcal{F}(M)^{*}$ be defined by $\langle\delta(x), \Lambda\rangle=g(x)$ for any $x \in M$. Since the $\delta(x)$ are linearly independent and linearly dense in $\mathcal{F}(M)$, this defines an element of $\mathcal{F}(M)^{*}$ properly, and it is obvious that $T(\Lambda)=g$. Thus $T$ is onto and $\mathcal{F}(M)^{*}$ is linearly isometric to $\operatorname{Lip}_{0}(M)$.

Finally, let $\left(f_{i}\right)$ be a net in $B_{\operatorname{Lip}_{0}(M)}$. Then $f_{i} \stackrel{w^{*}}{\longrightarrow} f$ if and only if $\left\langle m, f_{i}\right\rangle \rightarrow\langle m, f\rangle$ for any $m \in \mathcal{F}(M)$. But $\delta(M)$ is linearly dense in $\mathcal{F}(M)$, so this is equivalent to $\left\langle m, f_{i}\right\rangle \rightarrow\langle m, f\rangle$ for $m \in \delta(M)$, i.e. to $f_{i}(x) \rightarrow f(x)$ for all $x \in M$. This ends the proof.

The Lipschitz-free space $\mathcal{F}(M)$ is regarded as the canonical predual of $\operatorname{Lip}_{0}(M)$. In fact, it is conjectured (but unknown at the time of writing) that $\mathcal{F}(M)$ is the only possible predual of $\operatorname{Lip}_{0}(M)$, however this fact has only been proved when $M$ is bounded or geodesic [54]. In any case, whenever we make reference to the 
weak* topology in $\operatorname{Lip}_{0}(M)$ we will invariably mean the weak topology induced by $\mathcal{F}(M)$.

We must observe at this point that the linear structure of $\mathcal{F}(M)$ is once again independent of the choice of base point of $M$. Indeed, it is easy to check that the linear isometry between the corresponding $\operatorname{Lip}_{0}(M)$ spaces described in Proposition 2.1.6 is pointwise-to-pointwise continuous, hence $w^{*}-w^{*}$-continuous by the Banach-Dieudonné theorem. Its preadjoint is then a linear isometry between the Lipschitz-free spaces. Let us remark here that only the linear properties of Lipschitz and Lipschitz-free spaces remain invariant under changes of base point. Their lattice-theoretic structure does not, for instance; we shall see examples of this in Chapters 3 and 4 .

There are two main properties of Lipschitz-free spaces that justify the attention they get in current research efforts. The first one is the fact that $\mathcal{F}(M)^{*} \cong \operatorname{Lip}_{0}(M)$ as we have just proved. The second (and main) one is the "universal" extension property that we are about to prove. It essentially means that any Lipschitz mapping from $M$ to a Banach space can be "extended" to a linear operator with domain $\mathcal{F}(M)$; this is understood as an extension insofar as $M$ is identified with its image $\delta(M)$ in $\mathcal{F}(M)$.

Theorem 2.2.4 (Universal extension property). Let $f: M \rightarrow X$ be a Lipschitz mapping into a Banach space $X$ such that $f(0)=0$. Then there is $F \in$ $\mathcal{L}(\mathcal{F}(M), X)$ such that $\|F\|=\|f\|_{L}$ and $F(\delta(x))=f(x)$ for any $x \in M$, i.e. the following diagram is commutative:

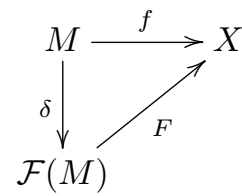

Proof. Define $F: \mathcal{F}(M) \rightarrow X$ by setting $F(\delta(x))=f(x)$ for $x \in M$ and extending linearly. Since $\{\delta(x): x \in X\}$ is linearly independent and linearly dense in $\mathcal{F}(M)$, this correctly defines a unique linear mapping $F$. Moreover, we have

$$
\begin{aligned}
\|F\|=\sup _{m \in B_{\mathcal{F}(M)}}\|F(m)\| & =\sup _{m \in B_{\mathcal{F}(M)}} \sup _{x^{*} \in B_{X^{*}}} x^{*}(F(m)) \\
& =\sup _{x^{*} \in B_{X^{*}}} \sup _{m \in B_{\mathcal{F}(M)}}\left(x^{*} \circ F\right)(m) \\
& =\sup _{x^{*} \in B_{X^{*}}}\left\|x^{*} \circ F\right\|_{\mathcal{F}(M)}
\end{aligned}
$$


since $x^{*} \circ F \in \mathcal{F}(M)^{*}$ for $x^{*} \in X^{*}$. Now notice that by Theorem 2.2.3

$$
\begin{aligned}
\left\|x^{*} \circ F\right\|_{\mathcal{F}(M)}=\left\|x^{*} \circ F \circ \delta\right\|_{\operatorname{Lip}_{0}(M)} & =\left\|x^{*} \circ f\right\|_{L} \\
& =\sup _{x \neq y \in M} \frac{\left(x^{*} \circ f\right)(x)-\left(x^{*} \circ f\right)(y)}{d(x, y)} \\
& =\sup _{x \neq y \in M} x^{*}\left(\frac{f(x)-f(y)}{d(x, y)}\right) .
\end{aligned}
$$

We now conclude that

$$
\|F\|=\sup _{x^{*} \in B_{X^{*}}} \sup _{x \neq y \in M} x^{*}\left(\frac{f(x)-f(y)}{d(x, y)}\right)=\sup _{x \neq y \in M}\left\|\frac{f(x)-f(y)}{d(x, y)}\right\|=\|f\|_{L} .
$$

In particular $F \in \mathcal{L}(\mathcal{F}(M), X)$. This ends the proof.

The authorship of Theorem 2.2.4 is somewhat disputed. The universal extension property can be found in the works of Kadets [38] and Pestov [46] in 1985-86, but was first stated in its current form by Weaver in 1999 in [52]. Each of these authors dealt with a different construction of the Lipschitz-free space.

Let us provide yet another way to express the conclusion of Theorem 2.2.4. Denote by $\operatorname{Lip}_{0}(M, X)$ the space of $X$-valued Lipschitz mappings on $M$ that vanish at the base point, which is easily shown to be a Banach space using the same argument as in Proposition 2.1.6. Then Theorem 2.2.4 asserts that $\operatorname{Lip}_{0}(M, X) \cong \mathcal{L}(\mathcal{F}(M), X)$, with a linear isometry given by composition with $\delta$. In particular, for $X=\mathbb{R}$ we have $\mathcal{L}(\mathcal{F}(M), \mathbb{R})=\mathcal{F}(M)^{*}$ and we recover Theorem 2.2.3.

An important particular case arises when the target space is a Lipschitz-free space itself. The theorem then says that any Lipschitz mapping between metric spaces can be extended to a linear operator between the corresponding Lipschitz-free spaces:

Corollary 2.2.5. Let $f: M \rightarrow N$ be a Lipschitz mapping between two pointed metric spaces such that $f\left(0_{M}\right)=0_{N}$. Then there is $F \in \mathcal{L}(\mathcal{F}(M), \mathcal{F}(N))$ such that $\|F\|=\|f\|_{L}$ and $F \circ \delta_{M}=\delta_{N} \circ f$, i.e. the following diagram is commutative:

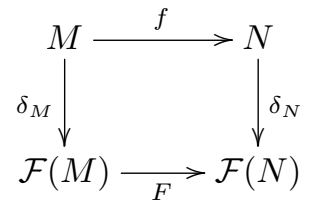

Proof. Apply Theorem 2.2.4 to the Lipschitz mapping $\delta_{N} \circ f: M \rightarrow \mathcal{F}(N)$. This yields an operator $F \in \mathcal{L}(\mathcal{F}(M), \mathcal{F}(N))$ that makes the diagram commutative and such that $\|F\|=\left\|\delta_{N} \circ f\right\|_{L}$. Finally notice that $\left\|\delta_{N} \circ f\right\|_{L}=\|f\|_{L}$ by Proposition 2.1.2, as $\delta_{N}$ is an isometry. 
Yet another application of the universal extension property allows us to show that Lipschitz-free spaces over subsets of $M$ may be identified as closed subspaces of $\mathcal{F}(M)$ :

Theorem 2.2.6. Let $K$ be a closed subset of $M$ that contains 0 . Then $\mathcal{F}(K)$ is linearly isometric to $\overline{\operatorname{span}} \delta(K) \subset \mathcal{F}(M)$.

Proof. Apply Theorem 2.2.4 to the restricted mapping $\delta_{M}: K \rightarrow \mathcal{F}(M)$ to obtain a linear operator $T: \mathcal{F}(K) \rightarrow \mathcal{F}(M)$ such that the following diagram is commutative

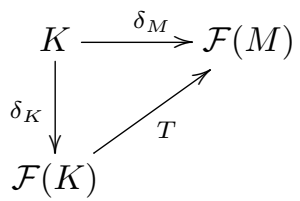

i.e. such that $T\left(\delta_{K}(x)\right)=\delta_{M}(x)$ for any $x \in K$. Now let $m \in \operatorname{span} \delta_{K}(K)$, say $m=\sum_{i=1}^{n} a_{i} \delta_{K}\left(x_{i}\right)$ where $a_{i} \in \mathbb{R}$ and $x_{i} \in K$. Then $T(m)=\sum_{i=1}^{n} a_{i} \delta_{M}\left(x_{i}\right)$, and we have

$$
\begin{aligned}
& \|m\|=\sup _{f \in B_{\mathrm{Lip}_{0}(K)}}\langle m, f\rangle=\sup _{f \in B_{\mathrm{Lip}_{0}(K)}} \sum_{i=1}^{n} a_{i} f\left(x_{i}\right) \\
& \|T(m)\|=\sup _{f \in B_{\mathrm{Lip}_{0}(M)}}\langle T(m), f\rangle=\sup _{f \in B_{\mathrm{Lip}_{0}(M)}} \sum_{i=1}^{n} a_{i} f\left(x_{i}\right)
\end{aligned}
$$

Notice that, by Corollary 2.1.10, $B_{\mathrm{Lip}_{0}(K)}$ consists precisely of the restrictions to $K$ of the functions in $B_{\operatorname{Lip}_{0}(M)}$. Therefore both suprema have the same value, and $\|T(m)\|=\|m\|$. Since this is true for $m$ in a dense subset of $\mathcal{F}(K), T$ must be an isometry, and it is clear that its range is $T\left(\overline{\operatorname{span}} \delta_{K}(K)\right)=\overline{T\left(\operatorname{span} \delta_{K}(K)\right)}=$ $\overline{\operatorname{span}} \delta_{M}(K)$.

We shall denote the subspace appearing in Theorem 2.2.6 as

$$
\mathcal{F}_{M}(K)=\overline{\operatorname{span}} \delta(K)=\overline{\operatorname{span}}\{\delta(x): x \in K\},
$$

with the convention that $\operatorname{span} \varnothing=\{0\}$. The requirement that $0 \in K$ in Theorem 2.2.6 is just a technical condition to ensure that $K$ is pointed with the same base point as $M$, so that the identity mapping $K \rightarrow M$ leaves the base point invariant. Nevertheless, notice that $\mathcal{F}_{M}(K)=\mathcal{F}_{M}(K \cup\{0\})$ by definition, so we may always attach the base point to $K$ to get the same result. The Lipschitz-free space $\mathcal{F}(K)$ can be completely identified with the subspace $\mathcal{F}_{M}(K)$ of $\mathcal{F}(M)$ and we will often do so, but certain arguments appearing in this thesis require keeping track of the particular overspace $M$ under consideration and this notation will prove useful there. 


\subsection{Distinguished elements and examples}

\section{Elementary molecules}

In this section we will introduce some of the most important members of $\mathcal{F}(M)$ and its bidual $\mathcal{F}(M)^{* *}=\operatorname{Lip}_{0}(M)^{*}$. We have already presented the evaluation functionals $\delta(x)$ and their basic properties. Members of span $\delta(M)$, i.e. finite linear combinations of evaluation functionals, will be called elements of finite support, an intuitive designation that will be shown to be completely precise in Proposition 3.3.8. Among those, the following are arguably the most important:

Definition 2.3.1. Let $x, y$ be two different points of $M$. The elementary molecule, or simply molecule, ${ }^{1}$ on points $x$ and $y$ is the element of $\mathcal{F}(M)$ given by

$$
m_{x y}=\frac{\delta(x)-\delta(y)}{d(x, y)} .
$$

The set of all elementary molecules in $\mathcal{F}(M)$ will be denoted by $\operatorname{Mol}(M)$.

Our first observation is that molecules always have norm 1, since $\delta$ is an isometry. Notice moreover that

$$
\left\langle m_{x y}, f\right\rangle=\frac{f(x)-f(y)}{d(x, y)}
$$

for any $f \in \operatorname{Lip}_{0}(M)$, i.e. the action of $m_{x y}$ on $f$ corresponds to taking the incremental quotient of $f$ on the pair $x, y$ that is used for the determination of its Lipschitz constant. It is immediate then that $\|f\|_{L}=\sup \{\langle m, f\rangle: m \in \operatorname{Mol}(M)\}$, and the Hahn-Banach theorem implies easily that

$$
B_{\mathcal{F}(M)}=\overline{\operatorname{conv}} \operatorname{Mol}(M) .
$$

In fact, a stronger approximation result is valid: every element of $\mathcal{F}(M)$ can be represented as a series of molecules, and one may choose the sum of the coefficients to be arbitrarily close to the norm. There are several known proofs of this basic fact, we provide here a rather self-contained one.

Proposition 2.3.2. Let $m \in \mathcal{F}(M)$. Then for every $\varepsilon>0$ there exist a sequence $\left(a_{n}\right)$ in $\mathbb{R}$ and a sequence $\left(x_{n}, y_{n}\right)$ of pairs of different points in $M$ such that

$$
m=\sum_{n=1}^{\infty} a_{n} m_{x_{n} y_{n}}
$$

and $\sum_{n=1}^{\infty}\left|a_{n}\right|<\|m\|+\varepsilon$.

\footnotetext{
${ }^{1}$ For some authors, like Weaver in [53], only the term elementary molecule refers to elements of the form $m_{x y}$, whereas molecule refers to any element of finite support.
} 
Proof. Let $\widetilde{M}$ be the set of pairs of different points in $M$, and define the mapping $T: \ell_{1}(\widetilde{M}) \rightarrow \mathcal{F}(M)$ by letting $T e_{(x, y)}=m_{x y}$ and extending linearly on

span $\left\{e_{(x, y)}:(x, y) \in \widetilde{M}\right\}$; here $e_{(x, y)}$ denote the standard unit vectors of $\ell_{1}(\widetilde{M})$. Since molecules have norm 1 , it is clear that $T$ has norm 1 when restricted to finitely supported elements, so $\|T\|=1$. Now notice that

$$
B_{\mathcal{F}(M)}^{O} \subset B_{\mathcal{F}(M)}=\overline{\operatorname{conv}} \operatorname{Mol}(M) \subset \overline{T\left(B_{\ell_{1}(\widetilde{M})}\right)}=\overline{T\left(B_{\ell_{1}(\widetilde{M})}^{O}\right)}
$$

where $B_{X}^{O}$ denotes the open unit ball of $X$. By a standard open mapping theorem argument (e.g. [22, Lemma 2.24]) we actually have $B_{\mathcal{F}(M)}^{O} \subset T\left(B_{\ell_{1}(\widetilde{M})}^{O}\right)$. That is, if $\|m\|<1$ then there is an expression of the form (2.2) such that $\sum_{n=1}^{\infty}\left|a_{n}\right|<1$. This is clearly equivalent to the statement of the Proposition.

It is clear that for any such expression (2.2) one has

$$
\|m\| \leq \sum_{n=1}^{\infty}\left\|a_{n} m_{x_{n} y_{n}}\right\|=\sum_{n=1}^{\infty}\left|a_{n}\right| .
$$

For some elements it is even possible to find a series of molecules such that $\sum_{n=1}^{\infty}\left|a_{n}\right|=\|m\|$; for instance, if $m$ is finitely supported then one may assume that $M$ is finite and this follows from Proposition 2.3.2 by a finiteness argument. However, this is in general not possible. We refer to [8] for counterexamples and necessary conditions.

One final reason for the importance of elementary molecules is that they provide prototypical examples of extremal elements of $B_{\mathcal{F}(M)}$; it is in fact conjectured that they are the only examples of extremal elements. We shall consider this issue in depth in Chapter 5.

\section{Positive and majorizable elements}

An entirely different family of elements of $\mathcal{F}(M)$ (and its bidual) will be introduced now, determined by the order structure of $\operatorname{Lip}_{0}(M)$. Recall that an ordered vector space $X$ is a vector space with a partial order $\leq$ that is compatible with addition and with multiplication by non-negative scalars. An element $x \in X$ is then called positive if $x \geq 0$. The set of all positive elements of $X$ is called the positive cone of $X$ and denoted $X^{+}$. Note that the order in $X$ is completely determined by its positive cone $X^{+}$. When $X$ is a normed space, we will write $B_{X}^{+}$for its positive unit ball i.e. the set of positive elements of $B_{X}$.

We have already seen that $\operatorname{Lip}_{0}(M)$ is a lattice under the pointwise order, generated by the positive cone

$$
\operatorname{Lip}_{0}(M)^{+}=\left\{f \in \operatorname{Lip}_{0}(M): f(x) \geq 0 \text { for all } x \in M\right\} .
$$


Thus, any $f \geq 0$ will be called positive, even if the term non-negative might be more accurate. This order structure induces a partial order on $\operatorname{Lip}_{0}(M)^{*}$ that turns it into an ordered vector space: we say that the functional $\phi \in \operatorname{Lip}_{0}(M)^{*}$ is positive if $\langle f, \phi\rangle \geq 0$ for all $f \in \operatorname{Lip}_{0}(M)^{+}$. By restriction, this also endows $\mathcal{F}(M)$ with an ordered vector space structure. It should be remarked that neither $\mathcal{F}(M)$ nor $\operatorname{Lip}_{0}(M)^{*}$ are lattices in general (see the comment after Definition 2.3.5).

The norm of positive elements of $\operatorname{Lip}_{0}(M)^{*}$, including $\mathcal{F}(M)$, is particularly easy to compute and to handle:

Proposition 2.3.3. If $\phi \in \operatorname{Lip}_{0}(M)^{*}$ is positive, then $\|\phi\|=\langle\rho, \phi\rangle$. If $\left(\phi_{n}\right)$ are positive elements of $\operatorname{Lip}_{0}(M)^{*}$ and $\sum_{n} \phi_{n}$ is an absolutely convergent series, then $\left\|\sum_{n} \phi_{n}\right\|=\sum_{n}\left\|\phi_{n}\right\|$.

Proof. We have $\rho \in B_{\operatorname{Lip}_{0}(M)}$, and for any $f \in B_{\operatorname{Lip}_{0}(M)}$ we have $f \leq \rho$ and hence $\langle f, \phi\rangle \leq\langle\rho, \phi\rangle$. Taking the supremum over $f$ yields $\|\phi\|=\langle\rho, \phi\rangle$. The second assertion follows immediately by evaluating the series $\sum_{n} \phi_{n}$ on $\rho$.

Since any element of $\mathcal{F}(M)$ can be approximated in norm by elements of finite support, one may wonder whether positive elements of $\mathcal{F}(M)$ can also be approximated by positive elements of finite support, that is, linear combinations of evaluation functionals with positive coefficients. This is a folklore result and easy to prove. In fact, the same may be said for positive elements of $\operatorname{Lip}_{0}(M)^{*}$ replacing norm convergence with weak* convergence, and the proof of both facts is essentially the same:

Proposition 2.3.4. Every $m \in \mathcal{F}(M)^{+}$is the limit of a sequence $\left(m_{n}\right)$ of positive, finitely supported elements of $\mathcal{F}(M)$, and every $\phi \in\left(\mathcal{F}(M)^{* *}\right)^{+}$is the weak limit $^{*}$ of a net $\left(m_{i}\right)$ of positive, finitely supported elements of $\mathcal{F}(M)$.

Proof. We will only give the proof of the second statement. Let $A$ be the set of positive, finitely supported elements of $\mathcal{F}(M)$, and suppose that there exists $\phi \in\left(\mathcal{F}(M)^{* *}\right)^{+}$such that $\phi \notin \bar{A}^{w^{*}}$. By the Hahn-Banach separation theorem, there is $f \in \operatorname{Lip}_{0}(M)$ such that

$$
\langle f, \phi\rangle>\sup \left\{\langle f, \psi\rangle: \psi \in \bar{A}^{w^{*}}\right\} \geq 0
$$

In particular, taking $\psi=a \delta(x)$ for $a>0$ and $x \in M$, we get $a f(x)<\langle f, \phi\rangle$. Since this is true for any $a>0$, it follows that $f(x) \leq 0$. Hence $f \leq 0$, and the positivity of $\phi$ implies that $\langle f, \phi\rangle \leq 0$, a contradiction.

An important role will be played in later chapters by positive elements of $\mathcal{F}(M)$ and $\operatorname{Lip}_{0}(M)^{*}$, but also by the following, more general class of elements: 
Definition 2.3.5. Let $X$ be an ordered vector space and $x \in X$. A majorant of $x$ is a positive element $x^{+} \in X^{+}$such that $x \leq x^{+}$; we also say that $x$ is majorized by $x^{+}$. We will say that an element $x \in X$ is majorizable (more specifically, majorizable in $X$ ) if it has at least one majorant $x^{+} \in X^{+}$. Equivalently, $x$ is majorizable if it may be written as the difference $x=x^{+}-x^{-}$of two positive elements $x^{+}, x^{-}$of $X^{+}$.

Since $\operatorname{Lip}_{0}(M)$ is a lattice, every $f \in \operatorname{Lip}_{0}(M)$ is majorizable and can, in fact, be expressed canonically as $f=f^{+}-f^{-}$, where $f^{+}=f \vee 0$ and $f^{-}=(-f) \vee 0$ are positive and have Lipschitz constants bounded by $\|f\|_{L}$. However, not all elements of $\mathcal{F}(M)$ are majorizable in general, as illustrated e.g. by Example 3.24 in [53]; consequently, $\mathcal{F}(M)$ and $\operatorname{Lip}_{0}(M)^{*}$ are not lattices in general. In Theorem 4.4.2, we shall determine those metric spaces $M$ for which every element of $\mathcal{F}(M)$ is majorizable.

Notice that, for an element $m \in \mathcal{F}(M)$, there are two separate notions of majorizability: $m$ may be majorizable in $\mathcal{F}(M)$ or in $\operatorname{Lip}_{0}(M)^{*}$; that is, there may exist a positive $m^{+}$in $\mathcal{F}(M)$ such that $m \leq m^{+}$or there may exist a positive $\phi^{+}$in $\operatorname{Lip}_{0}(M)^{*}$ such that $m \leq \phi^{+}$. Formally, the latter is a weaker condition. It is by no means obvious whether both conditions are equivalent; they are, as a matter of fact, and we will prove that later in Theorem 4.3.15.

\section{Examples of Lipschitz-free spaces}

To finish this chapter, we will showcase some of the paradigmatic examples of Lipschitz and Lipschitz-free spaces for particular cases of the metric space $M$.

Example 2.3.6. Let $M$ be a finite metric space with $n+1$ elements, say $M=$ $\left\{0, x_{1}, \ldots, x_{n}\right\}$. Then $\operatorname{Lip}_{0}(M) \sim \mathbb{R}^{n}$. Indeed, one may identify $f \in \operatorname{Lip}_{0}(M)$ with the $n$-tuple $\left(f\left(x_{1}\right), \ldots, f\left(x_{n}\right)\right) \subset \mathbb{R}^{n}$ and, conversely, any such $n$-tuple defines a Lipschitz function as there are only finitely many incremental quotients to choose from. Since $\operatorname{Lip}_{0}(M)$ is finite dimensional, it must be reflexive and hence $\mathcal{F}(M)=$ $\operatorname{Lip}_{0}(M)^{*}$ is also isomorphic to $\mathbb{R}^{n}$.

Not every finite-dimensional space can be realized as a Lipschitz or Lipschitz-free space. For instance, the unit ball of $\operatorname{Lip}_{0}(M)$ is always a polyhedron, given by the intersection of finitely many half-spaces

$$
\left\{\left(a_{1}, \ldots, a_{n}\right) \in \mathbb{R}^{n}: a_{i}-a_{j} \leq d\left(x_{i}, x_{j}\right)\right\}
$$

for $i \neq j$ between 1 and $n$. As a consequence, the unit ball of $\mathcal{F}(M)$ is also a polyhedron. Moreover, in the first orthant, the norm of $\mathcal{F}(M)$ coincides with a weighted $\ell_{1}$ norm on $\mathbb{R}^{n}$. Indeed, those elements are exactly the positive elements of $\mathcal{F}(M)$, expressible as $m=\sum_{k=1}^{n} a_{k} \delta\left(x_{k}\right)$ where $a_{k} \geq 0$, therefore $\|m\|=$ $\sum_{k=1}^{n} a_{k} d\left(x_{k}, 0\right)$ by Proposition 2.3.3. In particular, $B_{\mathcal{F}(M)}$ has no vertexes in the 
interior of the first orthant (we shall prove later that this is true even for infinite $M$; see Theorem 5.3.4).

It is even possible to give an explicit formula for the norm of $\mathcal{F}(M)$ in terms of the metric of $M$. When $M=\{0, x, y\}$, the norm of an arbitrary element of $\mathcal{F}(M)$ is given by

$$
\|a \delta(x)+b \delta(y)\|= \begin{cases}d(x, 0)|a|+d(y, 0)|b| & , \text { if } a b \geq 0 \\ d(x, 0)|a|+(d(x, y)-d(y, 0))|b| & , \text { if } a b \leq 0 \text { and }|b| \leq|a| \\ (d(x, y)-d(x, 0))|a|+d(y, 0)|b| & , \text { if } a b \leq 0 \text { and }|a| \leq|b|\end{cases}
$$

(see [15, Lemma 11]). But already for $|M|=4$ the closed formula for the norm of $\mathcal{F}(M)$ is too complicated to handle.

We should mention here that $\mathcal{F}(M)$ and $\operatorname{Lip}_{0}(M)$ are reflexive only when $M$ is finite (see [53, Corollary 3.46]).

Example 2.3.7. Let $M=\mathbb{N} \cup\{0\}$ with the usual metric, inherited from $\mathbb{R}$. Then $\mathcal{F}(M) \cong \ell_{1}$ and $\operatorname{Lip}_{0}(M) \cong \ell_{\infty}$. Indeed, let us see that the linear mapping $T: \operatorname{Lip}_{0}(M) \rightarrow \ell_{\infty}$ defined by $(T f)_{n}=f(n)-f(n-1), n \in \mathbb{N}$ is a surjective isometry. Choose $f \in S_{\operatorname{Lip}_{0}(M)}$. It is clear that $|f(n)-f(n-1)| \leq 1$ for any $n \in \mathbb{N}$, hence $\|T f\|_{\infty} \leq 1$. On the other hand, for any $\varepsilon>0$ there are $m<n$ in $\mathbb{N} \cup\{0\}$ such that

$$
1-\varepsilon<\frac{|f(n)-f(m)|}{n-m} \leq \max _{m<k \leq n}|f(k)-f(k-1)|
$$

and therefore $\|T f\|_{\infty}>1-\varepsilon$. Thus $T$ is an isometry. It is also easy to check that, for any $\left(a_{n}\right) \in \ell_{\infty}$, the function $f$ given by $f(0)=0$ and $f(n)=\sum_{k=1}^{n} a_{k}$ for $n \in \mathbb{N}$ satisfies $\|f\|_{L} \leq\left\|\left(a_{n}\right)\right\|_{\infty}$ and $T f=\left(a_{n}\right)$. It follows that $\operatorname{Lip}_{0}(M) \cong \ell_{\infty}$, and since $\ell_{\infty}$ is known to have a unique predual (see e.g. [29]) we must have $\mathcal{F}(M) \cong \ell_{1}$. In fact, the isometry between these spaces is just the preadjoint of $T$; its inverse is $S: \mathcal{F}(M) \rightarrow \ell_{1}$ where $S(\delta(n))=\sum_{k=1}^{n} e_{n}$.

The following is an important related example:

Example 2.3.8. Let $M=\mathbb{N} \cup\{0\}$ and define a metric $d$ on $M$ as follows: for any $n \neq m \in \mathbb{N}$ let $d(n, m)=2$ and $d(n, 0)=1$. The space $(M, d)$ is usually called the equilateral space. Let us see that $\operatorname{Lip}_{0}(M)$ is once again linearly isometric to $\ell_{\infty}$ under the mapping $(T f)_{n}=f(n)$. Indeed, if $f \in S_{\operatorname{Lip}_{0}(M)}$ then

$$
|f(n)|=|f(n)-f(0)| \leq d(n, 0)=1
$$

for any $n \in \mathbb{N}$, so $\|T f\|_{\infty} \leq 1$. On the other hand, given $\varepsilon>0$ there are $m<n$ in $\mathbb{N} \cup\{0\}$ such that $|f(n)-f(m)|>(1-\varepsilon) d(n, m)$. If $m=0$ this implies $|f(n)|>$ $1-\varepsilon$, otherwise $|f(n)-f(m)|>2(1-\varepsilon)$ and so $\max \{|f(n)|,|f(m)|\}>1-\varepsilon$. 
So in any case $\|T f\|_{\infty}>1-\varepsilon$. Finally, if $\left(a_{n}\right) \in B_{\ell_{\infty}}$ then the function $f$ given by $f(0)=0$ and $f(n)=a_{n}$ is clearly in $B_{\operatorname{Lip}_{0}(M)}$ and $T f=\left(a_{n}\right)$. So we have $\operatorname{Lip}_{0}(M) \cong \ell_{\infty}$, and therefore also $\mathcal{F}(M) \cong \ell_{1}$ under the preadjoint mapping $S: \ell_{1} \rightarrow \mathcal{F}(M)$ given by $S\left(e_{n}\right)=\delta(n)$.

The previous examples all dealt with topologically discrete metric spaces. Let us now see what kind of Lipschitz-free spaces arise when the underlying metric space is connected:

Example 2.3.9. Let $M=[0,1]$ with the usual metric. Let us prove that $\operatorname{Lip}_{0}(M) \cong L_{\infty}[0,1]$ under the mapping $T: L_{\infty}[0,1] \rightarrow \operatorname{Lip}_{0}[0,1]$ given by

$$
(T f)(x)=\int_{0}^{x} f(t) d t
$$

for $f \in L_{\infty}[0,1]$. Indeed, for any such $f$ and $x<y$ in $[0,1]$ we have

$$
|(T f)(y)-(T f)(x)|=\left|\int_{x}^{y} f(t) d t\right| \leq\|f\|_{\infty}(y-x)
$$

and so $\|T f\|_{L} \leq\|f\|_{\infty}$ and $T f \in \operatorname{Lip}_{0}[0,1]$. Now consider the mapping $S$ : $\operatorname{Lip}_{0}[0,1] \rightarrow L_{\infty}[0,1]$ given by $S f=f^{\prime}$. Note that any Lipschitz function $f \in$ $\operatorname{Lip}_{0}[0,1]$ is absolutely continuous and therefore satisfies the fundamental theorem of calculus, i.e. $f^{\prime}$ exists almost everywhere and

$$
f(y)-f(x)=\int_{x}^{y} f^{\prime}(t) d t
$$

for any $x<y$ in $[0,1]$, that is, $T(S f)=f$ (this fact is also referred to as Rademacher's theorem). It is also clear that

$$
\left|f^{\prime}(x)\right|=\lim _{h \rightarrow 0}\left|\frac{f(x+h)-f(x)}{h}\right| \leq\|f\|_{L}
$$

for any $x \in[0,1]$ where $f^{\prime}(x)$ exists, hence $S f \in L_{\infty}[0,1]$ and $\|S f\|_{\infty} \leq\|f\|_{L}$. To recap, we have proved that $T$ and $S$ are inverses of each other and non-expansive, and it follows that they are surjective linear isometries.

Since $L_{\infty}$ spaces have unique preduals (again, see e.g. [29]), we conclude that $\mathcal{F}([0,1]) \cong L_{1}[0,1]$. The corresponding preadjoint linear isometry takes $\delta(x)(x \in$ $[0,1])$ to the characteristic function $\chi_{[0, x]}$.

One can use a similar argument to show that $\operatorname{Lip}_{0}(\mathbb{R}) \cong L_{\infty}(\mathbb{R})$ and $\mathcal{F}(\mathbb{R}) \cong$ $L_{1}(\mathbb{R})$. These facts, together with Example 2.3.7, admit the following generalization proved by Godard [28]: 
Theorem 2.3.10 (Godard). Let $M$ be a closed subset of $\mathbb{R}$. If $M$ has measure zero then $\mathcal{F}(M) \cong \ell_{1}$. Otherwise $\mathcal{F}(M) \cong L_{1} \oplus_{1} \ell_{1}^{n-1}$ where $n$ is the number of connected components of $M$ (with the convention that $\ell_{1}^{\infty}=\ell_{1}$ ).

This theorem already suggests that general Lipschitz and Lipschitz-free spaces are somehow related to $L_{\infty}$ and $L_{1}$ spaces, respectively. It is a good intuition to think about Lipschitz-free spaces as a generalization of $L_{1}$ spaces, and there are a number of positive results in this direction. For instance, every Lipschitz-free space $\mathcal{F}(M)$ contains a complemented copy of $\ell_{1}(\Gamma)$ where $\Gamma$ is the density character of $M$ [33], and the norm of $\mathcal{F}(M)$ is octahedral for most metric spaces $M$ [11]. However, there are also negative results. A celebrated result of Naor and Schechtman states that $\mathcal{F}\left(\mathbb{R}^{2}\right)$ cannot be isomorphically embedded into $L_{1}$ [45]. By virtue of Theorem 2.2.6, the same must be true of $\mathcal{F}\left(\mathbb{R}^{n}\right)$ for $n>2$; in particular, $\mathcal{F}\left(\mathbb{R}^{n}\right)$ is not isomorphic to $\mathcal{F}(\mathbb{R})$ for $n>1 .^{2}$ At the time of writing, it is an open problem whether $\mathcal{F}\left(\mathbb{R}^{m}\right)$ and $\mathcal{F}\left(\mathbb{R}^{n}\right)$ are isomorphic for any $n>m \geq 2$.

\footnotetext{
${ }^{2}$ This does not stop some papers from claiming otherwise [20].
} 



\section{Chapter 3}

\section{Supports in Lipschitz-free spaces and their biduals}

In this chapter we will introduce the notion of support of an element $m \in \mathcal{F}(M)$. Much like the analogous concept used when dealing with functions or measures, the support of $m$ represents the region of the metric space $M$ where $m$ has an effect; or conversely, $m$ completely ignores what happens outside of its support. This is a basic notion that can be successfully applied to the study of many problems in Lipschitz-free spaces but was unused until recently. The proof of the existence of supports first appeared in [5] for bounded $M$, and then in [6] for the general case.

To obtain these results, we will need to develop one particular tool that is of independent interest: weighting operators on Lipschitz-free spaces (and their biduals). They allow functionals over Lipschitz spaces to be modulated by a fixed Lipschitz function in much the same way as when dealing with measures; here, the weighting function plays a role analogous to that of a Radon-Nikodým derivative. As a stand-alone application of this tool, we solve a problem of Weaver asking whether all normal functionals in $\mathcal{F}(M)^{* *}$ belong to $\mathcal{F}(M)$. Further applications of supports and weighting will be given in the next two chapters.

Finally, we will extend the notion of support for functionals $\phi \in \mathcal{F}(M)^{* *}$. The proposed definition has slightly weaker properties. In this case, the support cannot be defined as a subset of $M$, but rather of a compactification of $M$. The most appropriate compactification to use in this context is the uniform compactification, which we shall also introduce. Additionally, this will allow us to describe a canonical decomposition of $\mathcal{F}(M)^{* *}$ into functionals "concentrated at infinity" and functionals "away from infinity". 


\subsection{Weighting operators}

Let us start this chapter by introducing a class of operators on Lipschitz spaces and their duals and preduals that will be used extensively throughout the rest of this document.

Definition 3.1.1. Let $h \in \operatorname{Lip}(M)$, and let $K$ be a closed subset of $M$ such that $\operatorname{supp}(h) \subset K$. We define the weighting operator $W_{h}$ associated to $h$ as the mapping $W_{h}: \operatorname{Lip}_{0}(K) \rightarrow C(M)$ given by

$$
W_{h}(f)(x)=\left\{\begin{array}{ll}
f(x) h(x) & \text { if } x \in K \\
0 & \text { if } x \notin K
\end{array} .\right.
$$

In the most general case $W_{h}(f)$ need not be a Lipschitz function. When $M$ is bounded, however, $\operatorname{Lip}(M)$ is closed under pointwise products by Proposition 2.1.3(d) and so $W_{h}(f) \in \operatorname{Lip}_{0}(M)$. This also holds in a slightly more general case:

Proposition 3.1.2. Suppose that $h \in \operatorname{Lip}(M)$ has bounded support, and let $K \subset M$ be closed and contain the base point and the support of $h$. Then $W_{h} \in$ $\mathcal{L}\left(\operatorname{Lip}_{0}(K), \operatorname{Lip}_{0}(M)\right)$ and

$$
\left\|W_{h}\right\| \leq\|h\|_{\infty}+\operatorname{rad}(\operatorname{supp}(h))\|h\|_{L} .
$$

Moreover $W_{h}$ is $w^{*}-w^{*}$-continuous, i.e. its adjoint $W_{h}^{*}$ takes $\mathcal{F}(M)$ into $\mathcal{F}(K)$.

Proof. Put $S=\operatorname{supp}(h)$. First, we show that $W_{h}(f) \in \operatorname{Lip}_{0}(M)$ for any $f \in$ $\operatorname{Lip}_{0}(K)$. Clearly $W_{h}(f)(0)=0$. If $x, y \in S$, then

$$
\begin{aligned}
\frac{\left|W_{h}(f)(x)-W_{h}(f)(y)\right|}{d(x, y)} & =\frac{|f(x) h(x)-f(y) h(y)|}{d(x, y)} \\
& \leq \frac{|f(x) h(x)-f(x) h(y)|}{d(x, y)}+\frac{|f(x) h(y)-f(y) h(y)|}{d(x, y)} \\
& \leq \sup _{S}|f| \cdot\|h\|_{L}+\|h\|_{\infty} \cdot\|f\|_{L} \\
& \leq\left(\operatorname{rad}(S)\|h\|_{L}+\|h\|_{\infty}\right)\|f\|_{L},
\end{aligned}
$$

and if $x \in S$ and $y \in M \backslash S$ then

$$
\begin{aligned}
\frac{\left|W_{h}(f)(x)-W_{h}(f)(y)\right|}{d(x, y)} & =\frac{|f(x) h(x)-f(x) h(y)|}{d(x, y)} \\
& \leq \sup _{S}|f| \cdot\|h\|_{L} \leq \operatorname{rad}(S)\|h\|_{L}\|f\|_{L}
\end{aligned}
$$


Therefore the function $W_{h}(f)$ is Lipschitz and

$$
\left\|W_{h}(f)\right\|_{L} \leq\left(\operatorname{rad}(S)\|h\|_{L}+\|h\|_{\infty}\right) \cdot\|f\|_{L} \cdot
$$

Hence, $W_{h}$ is a well defined and bounded operator from $\operatorname{Lip}_{0}(K)$ into $\operatorname{Lip}_{0}(M)$. Linearity is obvious.

Finally, we prove that $W_{h}$ is $w^{*}-w^{*}$-continuous. By the Banach-Dieudonné theorem, it suffices to show that it is $w^{*}-w^{*}$-continuous on bounded subsets of $\operatorname{Lip}_{0}(K)$. Since $w^{*}$-convergence agrees with pointwise convergence on bounded subsets of Lipschitz spaces, it is enough to verify that $W_{h}\left(f_{i}\right) \rightarrow W_{h}(f)$ pointwise whenever $\left(f_{i}\right)$ is a net in $B_{\operatorname{Lip}_{0}(K)}$ that converges pointwise to $f$. But this is immediate from the definition of $W_{h}$.

We will usually denote the action of the adjoint of $W_{h}$ on functionals $\phi \in \mathcal{F}(M)^{* *}$ as $W_{h}^{*}(\phi)=\phi \circ W_{h}$. This notation is consistent, since $\left\langle f, W_{h}^{*}(\phi)\right\rangle=\left\langle W_{h}(f), \phi\right\rangle=$ $\left(\phi \circ W_{h}\right)(f)$ for any $f \in \operatorname{Lip}_{0}(K)$. Notice that if $h \geq 0$ then $W_{h}$ takes positive functions to positive functions, and therefore $W_{h}^{*}$ takes positive functionals to positive functionals.

Let us also observe that the function $W_{h}(f)$ does not depend on the choice of $K$, as long as it contains $\operatorname{supp}(h)$. Thus the requirement that $0 \in K$ is not really a restriction, as one may always use the set $K \cup\{0\}$ instead. In most cases we will just take $K=M$ without further mention; we will always mention $K$ explicitly when it is a proper subset of $M$.

Weighting will primarily be used with Urysohn-lemma-like functions constructed in the following way. Let $A, B$ be two subsets of $M$ such that $d(A, B)>0$; $A$ represents a "region of interest" where we want to focus, and $B$ is a region that we want to ignore. By McShane's theorem, there is $h \in \operatorname{Lip}(M)$ such that $0 \leq h \leq 1, h=1$ in $A, h=0$ in $B$, and $\|h\|_{L} \leq 1 / d(A, B)$. If $A$ is bounded then one can choose $h$ with bounded support by replacing $B$ with the larger set $B^{\prime}=\{x \in M: d(x, A) \geq d(A, B)\}$. Then $W_{h}$ is an operator on $\operatorname{Lip}_{0}(M)$ such that

$$
\left\|W_{h}\right\| \leq 1+\operatorname{rad}(\operatorname{supp}(h))\|h\|_{L}
$$

and, for every $m \in \mathcal{F}(M)$, one has $\left\langle m \circ W_{h}, f\right\rangle=\langle m, f\rangle$ whenever $\operatorname{supp}(f) \subset A$ and $\left\langle m \circ W_{h}, f\right\rangle=0$ whenever $\operatorname{supp}(f) \subset B$. On the other hand, if $B$ is bounded then one may similarly choose $h$ such that $\operatorname{supp}(1-h)$ is bounded, then $W_{h}=$ $I-W_{1-h}$ is also an operator on $\operatorname{Lip}_{0}(M)$ with the same properties (here $I$ denotes the identity operator). In this case, its norm is bounded by

$$
\left\|W_{h}\right\| \leq\|I\|+\left\|W_{1-h}\right\| \leq 2+\operatorname{rad}(\operatorname{supp}(1-h))\|h\|_{L} .
$$

If $A$ and $B$ are both unbounded, it is not possible to construct such an operator in general. 


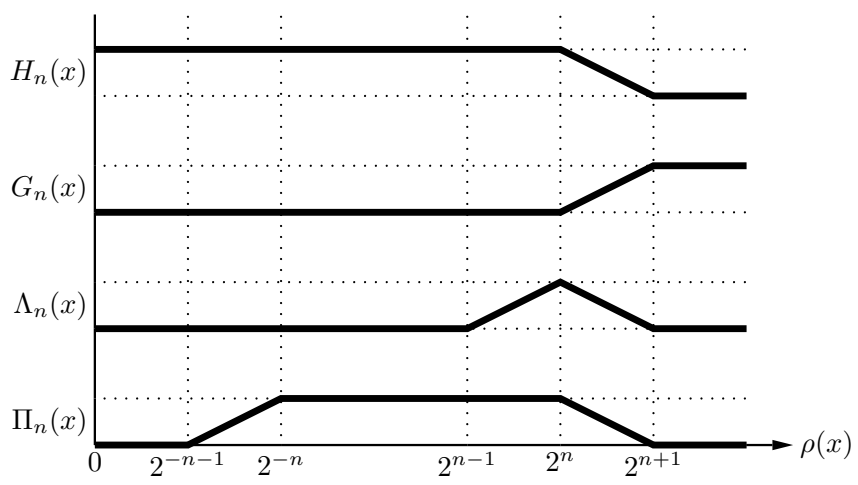

Figure 3.1: Standard weighting functions used in this thesis.

Let us now introduce several standard weighting functions that will be used often in this thesis, where the regions of interest are balls or annuli centered at the base point, or their complements. For $n \in \mathbb{Z}$, let

$$
\begin{gathered}
H_{n}(x)= \begin{cases}1 & , \text { if } \rho(x) \leq 2^{n} \\
2-2^{-n} \rho(x) & , \text { if } 2^{n} \leq \rho(x) \leq 2^{n+1} \\
0 & , \text { if } 2^{n+1} \leq \rho(x)\end{cases} \\
G_{n}(x)=1-H_{n}(x)
\end{gathered}
$$

and

$$
\Lambda_{n}(x)=G_{n-1}(x) H_{n}(x)
$$

and for $n \in \mathbb{N}$ let

$$
\Pi_{n}(x)=G_{-(n+1)}(x) H_{n}(x)=\sum_{k=-n}^{n} \Lambda_{k}(x) .
$$

All of these functions depend only on $\rho(x)$; their graphs are shown in Figure 3.1.

Notice that $\left\|H_{n}\right\|_{L} \leq 2^{-n}$ and $\operatorname{rad}\left(\operatorname{supp}\left(H_{n}\right)\right) \leq 2^{n+1}$, so (3.1) yields $\left\|W_{H_{n}}\right\| \leq 3$ and $\left\|W_{G_{n}}\right\| \leq 1+\left\|W_{H_{n}}\right\| \leq 4$. Similarly we get $\left\|W_{\Lambda_{n}}\right\| \leq 5$. Note also that

$$
\left\|W_{\Pi_{n}}\right\| \leq\left\|W_{G_{-(n+1)}}\right\| \cdot\left\|W_{H_{n}}\right\| \leq 12 .
$$

So all of these functions generate operators on $\operatorname{Lip}_{0}(M)$. Moreover, for every $f \in \operatorname{Lip}_{0}(M)$ the sequences of functions $\left(W_{H_{n}}(f)\right),\left(W_{G_{-n}}(f)\right)$ and $\left(W_{\Pi_{n}}(f)\right)$ (where $n \in \mathbb{N}$ ) are bounded and converge pointwise, hence weak ${ }^{*}$, to $f$, and if $f \geq 0$ then convergence is monotonic. Finally, notice that $W_{H_{m}} W_{H_{n}}=W_{H_{\min \{m, n\}}}$, $W_{G_{m}} W_{G_{n}}=W_{G_{\max \{m, n\}}}$ and $W_{\Pi_{m}} W_{\Pi_{n}}=W_{\Pi_{\min \{m, n\}}}$. 
To finish this section, we will give an application of these weighting operators that will be used later. In Section 4 of [40], Kalton established that elements of $\mathcal{F}(M)$ admit a decomposition as a series with terms whose action is limited to annuli around the base point. We will now prove a slightly different version of that decomposition based on the functions $\Lambda_{n}$ instead of the original ones because they make computations easier, and show that it can also be applied to certain functionals in $\mathcal{F}(M)^{* *}$.

Proposition 3.1.3. For any $\phi \in \mathcal{F}(M)^{* *}$, the functional

$$
\phi_{s}=\sum_{n \in \mathbb{Z}} \phi \circ W_{\Lambda_{n}}=\lim _{n \rightarrow \infty} \phi \circ W_{\Pi_{n}}
$$

belongs to $\mathcal{F}(M)^{* *}$, where the series converges absolutely and the limit is in norm.

Proof. We will prove that

$$
\sum_{n \in \mathbb{Z}}\left\|\phi \circ W_{\Lambda_{n}}\right\| \leq 45\|\phi\|
$$

which, together with the equality (3.5), implies the desired result. Fix $\varepsilon>0$ and a finite set $F \subset \mathbb{Z}$. For $i=0,1,2$, let $F_{i}$ be the set of those $n \in F$ that are congruent with $i$ modulo 3 . We will show that

$$
\sum_{n \in F_{i}}\left\|\phi \circ W_{\Lambda_{n}}\right\|<15\|\phi\|+\varepsilon
$$

and this will be enough to end the proof.

Fix $i$, and for $n \in F_{i}$ choose $f_{n} \in B_{\operatorname{Lip}_{0}(M)}$ such that

$$
\left\|\phi \circ W_{\Lambda_{n}}\right\|-\frac{\varepsilon}{\left|F_{i}\right|}<\left\langle f_{n}, \phi \circ W_{\Lambda_{n}}\right\rangle=\left\langle f_{n} \Lambda_{n}, \phi\right\rangle .
$$

Notice that $\left\|f_{n} \Lambda_{n}\right\|_{L} \leq\left\|W_{\Lambda_{n}}\right\| \leq 5$ by (3.1). Now define $g=\sum_{n \in F_{i}} f_{n} \Lambda_{n}$ and let us estimate $\|g\|_{L}$. Fix $x \in \operatorname{supp}(g)$, then $x \in \operatorname{supp}\left(\Lambda_{n}\right)$ for some $n \in F_{i}$. If $y \in \operatorname{supp}\left(\Lambda_{m}\right)$ for $m \in F_{i} \backslash\{n\}$, assume $m>n$ without loss of generality, then $d(x, y) \geq d(x, 0)$ and

$$
\begin{aligned}
|g(x)-g(y)| & \leq\left|f_{n}(x) \Lambda_{n}(x)\right|+\left|f_{m}(y) \Lambda_{m}(y)\right| \\
& \leq 5(d(x, 0)+d(y, 0)) \\
& \leq 5(2 d(x, 0)+d(x, y)) \leq 15 d(x, y) .
\end{aligned}
$$

Otherwise

$$
|g(x)-g(y)|=\left|f_{n}(x) \Lambda_{n}(x)-f_{n}(y) \Lambda_{n}(y)\right| \leq 5 d(x, y)
$$


So we get $\|g\|_{L} \leq 15$. Therefore

$$
\sum_{n \in F_{i}}\left\|\phi \circ W_{\Lambda_{n}}\right\|<\sum_{n \in F_{i}}\left\langle f_{n} \Lambda_{n}, \phi\right\rangle+\varepsilon=\langle g, \phi\rangle+\varepsilon \leq 15\|\phi\|+\varepsilon
$$

as was claimed.

In particular, if $\phi \in \mathcal{F}(M)$ then $\phi=\phi_{s}$ and we recover Kalton's decomposition result. Indeed, since $W_{\Pi_{n}}(f) \stackrel{w^{*}}{\longrightarrow} f$ for any $f \in \operatorname{Lip}_{0}(M)$, we have

$$
\left\langle\phi \circ W_{\Pi_{n}}, f\right\rangle=\left\langle\phi, W_{\Pi_{n}}(f)\right\rangle \rightarrow\langle\phi, f\rangle,
$$

i.e. $\phi \circ W_{\Pi_{n}} \stackrel{w}{\longrightarrow} \phi$, and Proposition 3.1.3 shows that we actually have convergence in the norm topology.

\subsection{Normal functionals}

In this section we will apply the previous results, in particular our version of Kalton's decomposition, to solve a problem regarding a certain property of functionals in $\mathcal{F}(M)^{* *}$. Before giving the definition let us recall that, by a straightforward application of the Banach-Dieudonné theorem, a functional $\phi \in \mathcal{F}(M)^{* *}$ is weak* continuous precisely when it satisfies the following condition: given any norm-bounded net $\left(f_{i}\right)$ in $\operatorname{Lip}_{0}(M)$ that converges pointwise to $f$, one has that $\left\langle f_{i}, \phi\right\rangle$ converges to $\langle f, \phi\rangle$.

In [52], Weaver introduced a weakened version of this property, by analogy with the corresponding notion for von Neumann algebras:

Definition 3.2.1. A functional $\phi \in \mathcal{F}(M)^{* *}$ is normal when it satisfies the following: given any norm-bounded net $\left(f_{i}\right)$ in $\operatorname{Lip}_{0}(M)$ that converges pointwise and monotonically to $f \in \operatorname{Lip}_{0}(M)$, one has that $\left\langle f_{i}, \phi\right\rangle$ converges to $\langle f, \phi\rangle$.

Equivalently, $\phi$ is normal if $\left\langle f_{i}, \phi\right\rangle \rightarrow 0$ for any net $\left(f_{i}\right)$ of non-negative functions in $B_{\operatorname{Lip}_{0}(M)}$ that decreases pointwise to 0 .

Any element of $\mathcal{F}(M)$ is obviously normal. Weaver asked in [52, p. 37] whether the converse is also true. Some particular cases were already proved by him. First he gave an affirmative answer for the very specific case of evaluation functionals on elements of the Stone-Čech compactification of $M$ [52, Proposition 2.1.6] and for "generalized molecules" [53, Theorem 3.43], as an auxiliary result to prove that all preserved extreme points of $B_{\mathcal{F}(M)}$ are elementary molecules [51]. Later, he proved that all positive normal functionals are weak* continuous and used it to show that $\mathcal{F}(M)$ is the unique predual of $\operatorname{Lip}_{0}(M)$ under various assumptions [54]. 
We must mention here the well-known theorem that states (i.e. positive linear functionals) on a von Neumann algebra are normal if and only if they belong to its predual; see e.g. [48, Theorem 1.13.2]. Thus Weaver's problem asks whether the classical result for von Neumann algebras carries over to Lipschitz spaces. Let us also briefly mention that a positive answer to the problem could be considered as an abstract version of the Radon-Nikodým theorem for Lipschitz-free spaces; compare e.g. to [49, Theorem 8.7].

Such a positive answer will be provided in this section. Before giving the proof, we need a few simple preparatory results. We will start by verifying that Kalton's decomposition is valid for normal functionals:

Lemma 3.2.2. Suppose that $\phi \in \mathcal{F}(M)^{* *}$ is normal. Then $\phi=\phi_{s}$, where $\phi_{s}$ is given by (3.6).

Proof. It will suffice to show that $\left(\phi \circ W_{\Pi_{n}}\right)$ converges weak* to $\phi$, since Proposition 3.1.3 implies that the sequence converges in norm to $\phi_{s}$. That is, we need to show that $\left\langle f, \phi \circ W_{\Pi_{n}}\right\rangle \rightarrow\langle f, \phi\rangle$ for any $f \in \operatorname{Lip}_{0}(M)$; we may assume that $f \geq 0$, and the general case then follows by expressing $f=f^{+}-f^{-}$. So fix $f \in \operatorname{Lip}_{0}(M)^{+}$ and notice that $\left\|W_{\Pi_{n}}(f)\right\|_{L} \leq 12\|f\|_{L}$, and $W_{\Pi_{n}}(f)(x)$ converges pointwise and monotonically (increasing) to $f(x)$ for every $x \in M$. By the normality of $\phi$ we have

$$
\lim _{n \rightarrow \infty}\left\langle f, \phi \circ W_{\Pi_{n}}\right\rangle=\lim _{n \rightarrow \infty}\left\langle W_{\Pi_{n}}(f), \phi\right\rangle=\langle f, \phi\rangle .
$$

This ends the proof.

Moreover, each term in the decomposition series and in the limit in (3.6) is also normal:

Lemma 3.2.3. Suppose that $h \in \operatorname{Lip}(M)$ is positive and has bounded support. If $\phi \in \mathcal{F}(M)^{* *}$ is normal, then $\phi \circ W_{h}$ is normal.

Proof. Let $\left(f_{i}\right)$ be a bounded net in $\operatorname{Lip}_{0}(M)$ that decreases to 0 pointwise. Then $\left\|f_{i} h\right\|_{L} \leq\left\|W_{h}\right\|\left\|f_{i}\right\|_{L}$ is bounded by $(3.1)$, so $\left(f_{i} h\right)$ is also a bounded net that decreases to 0 pointwise. Since $\phi$ is normal, we have

$$
\lim _{i}\left\langle f_{i}, \phi \circ W_{h}\right\rangle=\lim _{i}\left\langle f_{i} h, \phi\right\rangle=0 .
$$

It follows that $\phi \circ W_{h}$ is normal, too.

We will also need to use the following simple but powerful lemma from [13], which is itself based on a weaker version found in [39].

Lemma 3.2.4 ([13, Lemma 1.5]). Let $\left(f_{n}\right)$ be a bounded sequence in $\operatorname{Lip}_{0}(M)$. Suppose that the supports of the functions $f_{n}$ are pairwise disjoint. Then $f_{n} \stackrel{w}{\longrightarrow} 0$. 
We are now ready to answer Weaver's question.

Theorem 3.2.5. A functional $\phi \in \mathcal{F}(M)^{* *}$ is normal if and only if it is weak* continuous.

Proof. The sufficiency part of the statement is obvious. To prove the necessity, let $\phi \in \mathcal{F}(M)^{* *}$ be a normal functional. Lemma 3.2 .2 says that $\phi=\lim _{n \rightarrow \infty} \phi \circ W_{\Pi_{n}}$ with respect to the norm convergence, so it suffices to show that $\phi \circ W_{\Pi_{n}}$, for any $n \in \mathbb{N}$, is weak* continuous. Moreover, by Lemma 3.2.3, such $\phi \circ W_{\Pi_{n}}$ for any $n \in \mathbb{N}$ is also normal. Therefore it suffices to prove the theorem for functionals of the form $\phi \circ W_{\Pi_{n}}$.

Consequently, for the rest of the proof we will assume that $\phi \in B_{\mathcal{F}(M)^{* *}}$ is a normal functional and that there exist real numbers $0<r<R$ such that $\langle f, \phi\rangle=0$ whenever $f \in \operatorname{Lip}_{0}(M)$ vanishes on the set

$$
K=\{x \in M: r \leq d(x, 0) \leq R\} .
$$

We will repeatedly make use of the function

$$
e(x)=\left(1-\frac{4}{r} d(x, K)\right) \vee 0 \quad \text { for all } x \in M,
$$

whose support is contained in

$$
K^{\prime}=\left\{x \in M: \frac{3}{4} r \leq \rho(x) \leq R+\frac{r}{4}\right\}
$$

and which equals 1 on $K$, and the function

$$
e^{\prime}(x)=\left(1-\frac{4}{r} d\left(x, K^{\prime}\right)\right) \vee 0 \quad \text { for all } x \in M,
$$

whose support is contained in

$$
K^{\prime \prime}=\left\{x \in M: \frac{r}{2} \leq \rho(x) \leq R+\frac{r}{2}\right\}
$$

and which equals 1 on $K^{\prime}$. These auxiliary functions will play the role of units on $K$ and $K^{\prime}$ and will be used to truncate and translate other functions, respectively. Note that $e, e^{\prime} \in \operatorname{Lip}_{0}(M)^{+}$with $\|e\|_{L},\left\|e^{\prime}\right\|_{L} \leq \frac{4}{r}$; in particular,

$$
\left|\left\langle e^{\prime}, \phi\right\rangle\right| \leq \frac{4}{r} \text {. }
$$

For brevity, denote $\alpha=2+(R+1) \frac{4}{r}$.

We will proceed by contradiction. Suppose that $\phi \notin \mathcal{F}(M)$. Then there exists $F \in B_{\mathcal{F}(M)^{* * *}}$ such that $\langle\phi, F\rangle=C>0$ and that $\langle m, F\rangle=0$ for every $m \in \mathcal{F}(M)$. Our argument relies on a construction presented in the following Claim: 
Claim 1. With the notation as above, for a given nonempty finite set $A \subset K^{\prime}$ and $0<\varepsilon<\min \left\{1, \frac{C r}{48}\right\}$, there exists a function $g: M \rightarrow \mathbb{R}$ satisfying the following:

(i) $g \in \operatorname{Lip}_{0}(M)^{+}$with $\|g\|_{L} \leq \alpha$,

(ii) $g(x) \leq 2 \varepsilon$ for every $x \in A$,

(iii) $g(x) \geq \varepsilon$ for every $x \in K^{\prime}$,

(iv) $g(x)=\varepsilon e^{\prime}(x)$ for every $x \in M \backslash K^{\prime}$; in particular, $\operatorname{supp}(g) \subset K^{\prime \prime}$ and $\|g\|_{\infty} \leq \alpha\left(R+\frac{r}{2}\right)$,

(v) $|\langle g, \phi\rangle| \geq \frac{C}{4}$.

Proof of Claim 1. Consider the weak* neighborhood $U$ of $F$ in $\mathcal{F}(M)^{* * *}$ given by $U=\left\{\Psi \in \mathcal{F}(M)^{* * *}:|\langle\phi, F-\Psi\rangle|<\frac{C}{3}\right.$ and $|\langle\delta(x), F-\Psi\rangle|<\varepsilon$ for all $\left.x \in A\right\}$.

By Goldstine's theorem there exists $f \in B_{\operatorname{Lip}_{0}(M)} \cap U$, which means that $\left.\langle f, \phi\rangle\right\rangle$ $\frac{2 C}{3}$ and $|f(x)|=|\langle\delta(x), f\rangle|<\varepsilon$ for every $x \in A$. By replacing $f$ with $f^{+}$or $f^{-}$, we obtain a positive $f \in B_{\operatorname{Lip}_{0}(M)}$ such that

$$
|\langle f, \phi\rangle|>\frac{C}{3}
$$

and $f(x)<\varepsilon$ for every $x \in A$. Now let

$$
g=f e+\varepsilon e^{\prime} .
$$

Then $g \in \operatorname{Lip}_{0}(M)^{+}$and

$$
\|g\|_{L} \leq\|f\|_{L}\|e\|_{\infty}+\| f\left\lceil_{K^{\prime}}\left\|_{\infty}\right\| e\left\|_{L}+\varepsilon\right\| e^{\prime} \|_{L} \leq 1+\left(R+\frac{r}{4}\right) \frac{4}{r}+\varepsilon \frac{4}{r} \leq \alpha,\right.
$$

so $g$ satisfies (i). Moreover, $\operatorname{supp}(f e) \subset \operatorname{supp}(e) \subset K^{\prime}$, which establishes (iv). In particular, the bound on $\|g\|_{\infty}$ then follows from (i) and the definition of $K^{\prime \prime}$. Properties (ii) and (iii) are straightforward to verify. Finally, for every $x \in K$ we have that $g(x)=f(x)+\varepsilon$, that is $g \uparrow_{K}=\left(f+\varepsilon e^{\prime}\right) \uparrow_{K}$. Hence, by the assumption on $\phi$ and by (3.9) and (3.8), we get that

$$
|\langle g, \phi\rangle|=\left|\left\langle f+\varepsilon e^{\prime}, \phi\right\rangle\right| \geq|\langle f, \phi\rangle|-\varepsilon\left|\left\langle e^{\prime}, \phi\right\rangle\right|>\frac{C}{3}-\frac{C}{12}=\frac{C}{4}
$$

and (v) also holds.

To proceed with the main proof, let us fix a decreasing sequence $\left(\varepsilon_{n}\right)$ of positive numbers such that $\varepsilon_{1}<\min \left\{1, \frac{c r}{48}, \frac{r}{2}\right\}, \varepsilon_{n} \rightarrow 0$ and

$$
(2+\alpha) \varepsilon_{n+1}<\varepsilon_{n}
$$


for every $n$. Let $\mathfrak{F}$ be the family of all nonempty finite subsets of $K^{\prime}$, and for $A \in \mathfrak{F}$ let $\mathfrak{F}_{A}=\{B \in \mathfrak{F}: A \subset B\}$. Note that the sets $\mathfrak{F}$ and $\mathfrak{F}_{A}$ are directed by inclusion. We will now construct a net $\left(g_{A}\right)_{A \in \mathfrak{F}}$ in $\operatorname{Lip}_{0}(M)$ that satisfies conditions (i)-(iv) above with $\varepsilon=\varepsilon_{|A|}$, and also these two:

(vi) $\left|\left\langle g_{A}, \phi\right\rangle\right| \geq \frac{C}{8}$

(vii) if $E \subset A$ then $g_{A}(x) \leq g_{E}(x)$ for every $x \in M$.

This will be enough to end the proof. Indeed, $\left(g_{A}\right)_{A \in \mathfrak{F}}$ then decreases pointwise to 0 because $g_{A}(x) \leq 2 \varepsilon_{n}$ whenever $|A| \geq n$ and either $x \in A$ (by (ii)) or $x \in M \backslash K^{\prime}$ (by (iv)), but $\left|\left\langle g_{A}, \phi\right\rangle\right| \geq \frac{C}{8}$ for every $A \in \mathfrak{F}$, contradicting the normality of $\phi$.

We proceed by induction on $n=|A|$. For $n=1$ i.e. singletons $A=\{x\}$ with $x \in K^{\prime}$, let $g_{A}$ be the function $g$ given by Claim 1 for $\varepsilon=\varepsilon_{1}$. It clearly satisfies (i)-(vi), and also (vii) by vacuity. Now let $n>1$, assume that the functions $g_{A}$ have been constructed for all nonempty subsets $A \subset K^{\prime}$ with less than $n$ elements, and fix $A \subset K^{\prime}$ with $|A|=n$. To complete the induction, it suffices to prove that there exists $g_{A}$ satisfying (i)-(iv) and (vi)-(vii) with $\varepsilon=\varepsilon_{n}$.

Assume for contradiction that such a $g_{A}$ does not exist. That is, assume that any function $g$ that satisfies conditions (i)-(iv) and (vii) for $A$ and $\varepsilon=\varepsilon_{|A|}$ must fail (vi), i.e. it must be such that $|\langle g, \phi\rangle|<\frac{C}{8}$. Under this hypothesis, we can prove the following Claim:

Claim 2. If $A$ is as above and $n=|A|$, then there is a constant $\beta>0$ with the following property: for any $B \in \mathfrak{F}_{A}$, there exist $E \in \mathfrak{F}_{B}$ and $f \in \operatorname{Lip}_{0}(M)^{+}$such that

(a) $\|f\|_{L} \leq \beta$,

(b) $\operatorname{supp}(f) \subset\left(\bigcup_{x \in E} B\left(x, \varepsilon_{n}\right)\right) \backslash\left(\bigcup_{x \in B} B\left(x, \varepsilon_{n}\right)\right)$,

(c) $|\langle f, \phi\rangle| \geq \frac{C}{16}$.

Proof. Let $B \in \mathfrak{F}_{A}$ and let $g$ be the function given by Claim 1 for the set $B$ and $\varepsilon=\varepsilon_{n}$. Denote $h=\bigwedge_{E \subset A} g_{E}$, which satisfies conditions (i)-(iv) with $A$ and $\varepsilon=\varepsilon_{n-1}$. Notice that the function $g \wedge h$ satisfies conditions (i)-(iv) for $\varepsilon=\varepsilon_{n}$ and set $A$ because it is bounded by $g$, and also condition (vii) because it is bounded by $h$. By assumption, $g \wedge h$ must fail condition (vi), i.e. we have $|\langle g \wedge h, \phi\rangle|<\frac{C}{8}$.

Now let $f=(g-(g \wedge h)) \cdot e$. Clearly $f \geq 0$ and $\|f\|_{L} \leq 2 \alpha\left(2+\frac{4}{r} R\right)$ by (3.1). Suppose that $x \in B\left(b, \varepsilon_{n}\right)$ for some $b \in B$. If $x \notin K^{\prime}$ then $e(x)=0$, and if $x \in K^{\prime}$ then by (3.10) we have

$$
g(x) \leq g(b)+|g(x)-g(b)| \leq 2 \varepsilon_{n}+\alpha \varepsilon_{n}<\varepsilon_{n-1}
$$


whereas $h(x) \geq \varepsilon_{n-1}$, so $g(x) \leq h(x)$. In any case $f(x)=0$ for all $x \in$ $\bigcup_{b \in B} B\left(b, \varepsilon_{n}\right)$. Moreover,

$$
|\langle f, \phi\rangle|=|\langle g-(g \wedge h), \phi\rangle| \geq|\langle g, \phi\rangle|-|\langle g \wedge h, \phi\rangle|>\frac{C}{4}-\frac{C}{8}=\frac{C}{8} .
$$

Similarly to functions $e$ and $e^{\prime}$ introduced above, for a given $E \in \mathfrak{F}_{B}$ define the function

$$
e_{E}(x)=\left(1-\frac{1}{\varepsilon_{n}} d(x, E)\right) \vee 0 \quad \text { for all } x \in M .
$$

Then $e_{E} \in \operatorname{Lip}_{0}(M)^{+}$satisfies that $\left\|e_{E}\right\|_{L} \leq \frac{1}{\varepsilon_{n}},\left\|e_{E}\right\|_{\infty}=1$, and $\operatorname{supp}\left(e_{E}\right) \subset$ $\bigcup_{x \in E} B\left(x, \varepsilon_{n}\right)$. The net $\left(f e_{E}\right)_{E \in \mathfrak{F}_{B}}$ is a norm-bounded increasing net in $\operatorname{Lip}_{0}(M)^{+}$ that converges pointwise to $f$. Indeed, by (3.1) we have $\left\|f e_{E}\right\|_{L} \leq \beta$ where

$$
\beta=2 \alpha\left(2+R \frac{4}{r}\right)\left(1+\frac{1}{\varepsilon_{n}}\left(R+\frac{r}{4}\right)\right)
$$

does not depend on $B$ or $E$, and the rest is immediate from the definition. Hence the normality of $\phi$ implies that $\left\langle f e_{E}, \phi\right\rangle$ converges to $\langle f, \phi\rangle$, and in particular there exists $E \in \mathfrak{F}_{B}$ such that

$$
\left|\langle f, \phi\rangle-\left\langle f e_{E}, \phi\right\rangle\right|<\frac{C}{16} .
$$

The function $f e_{E}$ satisfies the requirements of the Claim. Indeed, we have already verified (a), (b) follows from $\operatorname{supp}\left(f e_{E}\right) \subset \operatorname{supp}(f) \cap \operatorname{supp}\left(e_{E}\right)$, and we get (c) from

$$
\left|\left\langle f e_{E}, \phi\right\rangle\right| \geq|\langle f, \phi\rangle|-\left|\langle f, \phi\rangle-\left\langle f e_{E}, \phi\right\rangle\right|>\frac{C}{8}-\frac{C}{16}=\frac{C}{16} .
$$

This ends the proof.

To conclude our main argument, let us construct sequences $\left(B_{n}\right) \subset \mathfrak{F}_{A}$ and $\left(f_{n}\right) \subset$ $\operatorname{Lip}_{0}(M)^{+}$as follows: take $B_{0}=A$, and for any $n \in \mathbb{N}$ let $B_{n}$ and $f_{n}$ be the set $E$ and function $f$ given by Claim 2 for $B=B_{n-1}$, respectively. Then the sequence $\left(f_{n}\right)$ is norm-bounded by (a) and has pairwise disjoint supports by (b). However it is not weakly null due to (c), which is in contradiction with Lemma 3.2.4. This ends the proof of Theorem 3.2.5.

\subsection{Supports in $\mathcal{F}(M)$}

We will establish the existence of supports in this section. It is based on (in fact, equivalent to) a rather simple and intuitive fact: the class of Lipschitz-free spaces over closed subsets of $M$, considered as subspaces of $\mathcal{F}(M)$, is closed under intersections. In order to prove this, we will need to obtain some results about the algebra structure of $\operatorname{Lip}_{0}(M)$ first. 
Ideals in $\operatorname{Lip}_{0}(M)$

Recall that $\operatorname{Lip}_{0}(M)$ is closed under pointwise products when $M$ is bounded, which endows it with an algebra structure. We will require some facts about its ideals. A subalgebra $Y \subset \operatorname{Lip}_{0}(M)$ is an ideal when it has the following property: for every $f \in Y$ and $g \in \operatorname{Lip}_{0}(M)$, one has $f g \in Y$. That is, $Y$ is not only closed under products, but also "absorbs" outside elements.

The most important class of ideals in $\operatorname{Lip}_{0}(M)$ is defined as follows. Let $K \subset M$, and denote

$$
\mathcal{I}_{M}(K)=\left\{f \in \operatorname{Lip}_{0}(M): f(x)=0 \text { for all } x \in K\right\} .
$$

In general, $\mathcal{I}_{M}(K)$ is a $w^{*}$-closed subspace of $\operatorname{Lip}_{0}(M)$ (being the intersection of the kernel of all evaluation functionals $\delta(x)$ for $x \in K)$ and $\mathcal{I}_{M}(K)=\mathcal{I}_{M}(\bar{K})$ so we may assume that $K$ is closed without loss of generality. Moreover $\mathcal{I}_{M}(K)=$ $\mathcal{I}_{M}(K \cup\{0\})$ as every function in $\operatorname{Lip}_{0}(M)$ vanishes at 0 ; in particular $\mathcal{I}_{M}(\{0\})=$ $\operatorname{Lip}_{0}(M)$. This may be remindful of the situation with Lipschitz-free subspaces $\mathcal{F}_{M}(K)$; that is not a coincidence, as they are strongly related to ideals:

Proposition 3.3.1. Let $K$ be a closed subset of $M$. Then $\mathcal{F}_{M}(K)^{\perp}=\mathcal{I}_{M}(K)$ and $\mathcal{I}_{M}(K)_{\perp}=\mathcal{F}_{M}(K)$.

Proof. Since the set $\delta(K)$ is linearly dense in $\mathcal{F}_{M}(K)$, we have $\mathcal{F}_{M}(K)^{\perp}=\delta(K)^{\perp}$, which is clearly identical to $\mathcal{I}_{M}(K)$ by definition. So $\mathcal{F}_{M}(K)^{\perp}=\mathcal{I}_{M}(K)$, and it follows that

$$
\mathcal{I}_{M}(K)_{\perp}=\left(\mathcal{F}_{M}(K)^{\perp}\right)_{\perp}=\overline{\operatorname{span}} \mathcal{F}_{M}(K)=\mathcal{F}_{M}(K)
$$

proving the other equality.

The space $\mathcal{I}_{M}(K)$ clearly absorbs products whenever they belong to $\operatorname{Lip}_{0}(M)$. In particular, it is an ideal when $M$ is bounded. It turns out that all $w^{*}$-closed ideals of $\operatorname{Lip}_{0}(M)$ are of this form. Let us first describe the natural way to recover the set $K$ from an arbitrary ideal: given a subspace $Y$ of $\operatorname{Lip}_{0}(M)$, define the hull of $Y$ as the closed set

$$
\mathcal{H}(Y)=\{x \in M: f(x)=0 \text { for all } f \in Y\} .
$$

Then we have $\mathcal{H}\left(\mathcal{I}_{M}(K)\right)=K$ for any closed $K \subset M$, as witnessed by the Lipschitz map $x \mapsto d(x, K)$. The "converse statement" is given by the following theorem, which combines several results from [53] (Theorem 7.17, Corollary 7.7 and the proof of Theorem 6.19 therein). ${ }^{1}$ We do not include its proof here, as it is a big detour and the details will not be needed in the sequel.

\footnotetext{
${ }^{1}$ The statement appeared explicitly in the first edition of the book as [52, Corollary 4.2.6].
} 
Theorem 3.3.2. Suppose that $M$ is bounded. If $Y$ is a $w^{*}$-closed ideal of $\operatorname{Lip}_{0}(M)$, then $Y=\mathcal{I}_{M}(\mathcal{H}(Y))$.

An application of the weighting operators introduced previously in this chapter allows us to show that the $w^{*}$-closure of any ideal in $\operatorname{Lip}_{0}(M)$ is again an ideal and hence of the form $\mathcal{I}_{M}(K)$ :

Proposition 3.3.3. Suppose that $M$ is bounded and let $Y \subset \operatorname{Lip}_{0}(M)$ be an ideal. Then $\bar{Y}^{w^{*}}=\mathcal{I}_{M}(\mathcal{H}(Y))$.

Proof. Let $f \in \bar{Y}^{w^{*}}, g \in \operatorname{Lip}_{0}(M)$ and $h=f g$. We claim that $h \in \bar{Y}^{w^{*}}$. Indeed, let $U \subset \operatorname{Lip}_{0}(M)$ be a $w^{*}$-neighborhood of $h$. Then $U$ contains a $w^{*}$-neighborhood $V$ of $h$ of the form

$$
V=\left\{\psi \in \operatorname{Lip}_{0}(M):\left|\left\langle m_{n}, h-\psi\right\rangle\right|<\varepsilon \text { for } n=1, \ldots, N\right\},
$$

where $m_{n} \in \mathcal{F}(M), \varepsilon>0$, and $N \in \mathbb{N}$. Consider the set

$$
W=\left\{\varphi \in \operatorname{Lip}_{0}(M):\left|\left\langle m_{n} \circ W_{g}, f-\varphi\right\rangle\right|<\varepsilon \text { for } n=1, \ldots, N\right\} .
$$

Since each $m_{n} \circ W_{g}$ is in $\mathcal{F}(M), W$ is a $w^{*}$-neighborhood of $f$ so there exists $\varphi \in Y \cap W$. Let $\psi=\varphi g$. Then $\psi \in Y$ because $Y$ is an ideal, and for any $n=1, \ldots, N$ we have

$$
\left|\left\langle m_{n}, h-\psi\right\rangle\right|=\left|\left\langle m_{n},(f-\varphi) g\right\rangle\right|=\left|\left\langle m_{n} \circ W_{g}, f-\varphi\right\rangle\right|<\varepsilon,
$$

so $\psi \in V \subset U$. Therefore $U \cap Y$ is nonempty, and this proves our claim.

We have thus proved that $\bar{Y}^{w^{*}}$ is an ideal. To conclude, apply Theorem 3.3.2 to get $\bar{Y}^{w^{*}}=\mathcal{I}_{M}\left(\mathcal{H}\left(\bar{Y}^{w^{*}}\right)\right)$. Clearly $\mathcal{H}\left(\bar{Y}^{w^{*}}\right)=\mathcal{H}(Y)$, and this ends the proof.

\section{The intersection theorem}

Let us highlight a simple fact about the role of Lipschitz functions with bounded support:

Lemma 3.3.4. The Lipschitz functions with bounded support are $w^{*}$-dense in $\operatorname{Lip}_{0}(M)$ and in $\mathcal{I}_{M}(K)$ for any $K \subset M$.

Proof. Fix $f \in \operatorname{Lip}_{0}(M)$ and consider the sequence of functions $f_{n}=W_{H_{n}}(f)$, where $H_{n}$ is defined in (3.2). Each of them satisfies $\left\|f_{n}\right\|_{L} \leq\left\|W_{H_{n}}\right\|\|f\|_{L} \leq$ $3\|f\|_{L}$ and $f_{n}(x)=f(x)$ for every $x \in B\left(0,2^{n}\right)$, hence $f_{n} \stackrel{w^{*}}{\longrightarrow} f$. Moreover $\operatorname{supp}\left(f_{n}\right) \subset \operatorname{supp}\left(H_{n}\right) \subset B\left(0,2^{n+1}\right)$ is bounded. Finally, notice that $f_{n}(x)=0$ 
whenever $f(x)=0$, hence if $f \in \mathcal{I}_{M}(K)$ then $f_{n} \in \mathcal{I}_{M}(K)$. It follows that the Lipschitz functions with bounded support are $w^{*}$-dense in $\mathcal{I}_{M}(K)$. In particular (taking $K=\{0\}$ ) they are $w^{*}$-dense in $\operatorname{Lip}_{0}(M)$.

In fact, we have proved something stronger: the Lipschitz functions with bounded support are 3 -norming for $\mathcal{F}(M)$. Recall that, given a Banach space $X$, a set $A \subset X^{*}$ is $C$-norming if

$$
\sup \left\{\left\langle x, x^{*}\right\rangle: x \in B_{X^{*}} \cap A\right\} \geq \frac{1}{C}\|x\|
$$

for any $x \in X$. A more precise construction shows that boundedly supported Lipschitz functions are even 1-norming for $\mathcal{F}(M)$. This will be proved as part of Theorem 3.5.3.

We now arrive at our main result, the intersection theorem:

Theorem 3.3.5 (Intersection theorem). Let $\left\{K_{i}: i \in I\right\}$ be a family of closed subsets of $M$. Then

$$
\bigcap_{i \in I} \mathcal{F}_{M}\left(K_{i}\right)=\mathcal{F}_{M}\left(\bigcap_{i \in I} K_{i}\right)
$$

Proof. Let $Y=\operatorname{span} \bigcup_{i \in I} \mathcal{I}_{M}\left(K_{i}\right)$. We will show that $\bar{Y}^{w^{*}}=\mathcal{I}_{M}(K)$ where $K=\bigcap_{i \in I} K_{i}$. This is enough, as Proposition 3.3.1 implies then that

$$
\begin{aligned}
\bigcap_{i \in I} \mathcal{F}_{M}\left(K_{i}\right) & =\bigcap_{i \in I} \mathcal{I}_{M}\left(K_{i}\right)_{\perp}=\left(\bigcup_{i \in I} \mathcal{I}_{M}\left(K_{i}\right)\right)_{\perp} \\
& =Y_{\perp}=\left(\bar{Y}^{w^{*}}\right)_{\perp}=\mathcal{I}_{M}(K)_{\perp}=\mathcal{F}_{M}(K) .
\end{aligned}
$$

Assume first that $M$ is bounded. Then $Y$ is an ideal and we may apply Proposition 3.3.3 to get $\bar{Y}^{w^{*}}=\mathcal{I}_{M}(H)$ where $H=\mathcal{H}(Y)$. Now notice that $\bigcap_{i \in I} K_{i} \subset H$, and for each $x \notin \bigcap_{i \in I} K_{i}$ there exists $i \in I$ such that $x \notin K_{i}$, so the function $y \mapsto d\left(y, K_{i}\right)$ belongs to $Y$ and witnesses that $x \notin H$. Thus $H=\bigcap_{i \in I} K_{i}$, and this settles the bounded case.

When $M$ is unbounded, we cannot use Proposition 3.3.3 because $Y$ is not necessarily closed under products. Instead, we will reduce the problem to the bounded case. Let us first notice that the inclusion $\bar{Y}^{w^{*}} \subset \mathcal{I}_{M}(K)$ is clear, so we only need to prove the reverse inclusion. Take $f \in \mathcal{I}_{M}(K)$ and let $U$ be a $w^{*}$-neighborhood of $f$ in $\operatorname{Lip}_{0}(M)$; it will suffice to show that $U$ intersects $Y$. 
We may assume that $f$ has bounded support by Lemma 3.3.4. So let $S=\operatorname{supp}(f)$, define $h \in \operatorname{Lip}(M)$ by

$$
h(x)=(1-d(x, S)) \vee 0
$$

for $x \in M$, and let $A=\operatorname{supp}(h) \cup\{0\}$. Thus $0 \leq h \leq 1, h \uparrow_{S}=1$ and $h=0$ outside of the bounded set $A$, so we may consider the continuous weighting operator $W_{h}: \operatorname{Lip}_{0}(A) \rightarrow \operatorname{Lip}_{0}(M)$. Let $\hat{f}=f \uparrow_{A} \in \operatorname{Lip}_{0}(A)$ and note that $W_{h}(\hat{f})=f$. Since $W_{h}$ is $w^{*}-w^{*}$-continuous by Proposition 3.1.2, there is a $w^{*}$-neighborhood $V$ of $\hat{f}$ such that $W_{h}(V) \subset U$.

Let us apply the bounded version of this theorem on the metric space $A$ to get

$$
\bigcap_{i \in I} \mathcal{F}_{A}\left(K_{i} \cap A\right)=\mathcal{F}_{A}(K \cap A) .
$$

It follows that

$$
\begin{aligned}
\mathcal{I}_{A}(K \cap A)=\left(\bigcap_{i \in I} \mathcal{F}_{A}\left(K_{i} \cap A\right)\right)^{\perp} & =\left(\left(\bigcup_{i \in I} \mathcal{I}_{A}\left(K_{i} \cap A\right)\right)_{\perp}\right)^{\perp} \\
& =\overline{\operatorname{span}}^{w^{*}} \bigcup_{i \in I} \mathcal{I}_{A}\left(K_{i} \cap A\right) .
\end{aligned}
$$

Now $\hat{f} \in \mathcal{I}_{A}(K \cap A)$, so there must exist $g \in V$ of the form $g=g_{1}+\ldots+g_{n}$ where $g_{k} \in \mathcal{I}_{A}\left(K_{i_{k}} \cap A\right), i_{k} \in I$ for $k=1, \ldots, n$. To finish the proof, note that $W_{h}\left(g_{k}\right) \in \mathcal{I}_{M}\left(K_{i_{k}}\right)$ for every $k=1, \ldots, n$ by the definition of $W_{h}$. Hence $W_{h}(g)=W_{h}\left(g_{1}\right)+\ldots+W_{h}\left(g_{n}\right) \in U \cap Y$.

With the intersection theorem at our disposal, we may finally introduce the main subject of this chapter:

Definition 3.3.6. Let $m \in \mathcal{F}(M)$. The support of $m$, denoted $\operatorname{supp}(m)$, is the intersection of all closed subsets $K$ of $M$ such that $m \in \mathcal{F}_{M}(K)$.

The most important property of supports, which makes them actually meaningful, is the following:

Theorem 3.3.7. For every $m \in \mathcal{F}(M)$ we have $m \in \mathcal{F}_{M}(\operatorname{supp}(m))$.

Indeed, let $\left\{K_{i}\right\}$ be the family of all closed subsets of $M$ such that $m \in \mathcal{F}_{M}\left(K_{i}\right)$, then by the intersection theorem we have

$$
m \in \bigcap_{i} \mathcal{F}_{M}\left(K_{i}\right)=\mathcal{F}_{M}\left(\bigcap_{i} K_{i}\right)=\mathcal{F}_{M}(\operatorname{supp}(m)) .
$$

In fact, Theorem 3.3.7 is an equivalent form of the intersection theorem, in the sense that the latter can be deduced from the statement of Theorem 3.3.7 just as easily. 


\section{Properties of supports}

Let us now collect some elementary properties of supports. First, we check that the choice of the name elements of finite support for finite linear combinations of evaluation functionals was completely appropriate:

Proposition 3.3.8. Let $m \in \mathcal{F}(M)$. Then $m \in \operatorname{span} \delta(M)$ if and only if $\operatorname{supp}(m)$ is a finite set. More precisely, if

$$
m=\sum_{i=1}^{n} a_{i} \delta\left(x_{i}\right)
$$

where $x_{i} \in M \backslash\{0\}$ are all different and $a_{i} \in \mathbb{R} \backslash\{0\}$, then $\operatorname{supp}(m)=\left\{x_{1}, \ldots, x_{n}\right\}$.

Proof. Suppose first that $\operatorname{supp}(m)$ is a finite set $K$, then

$$
m \in \mathcal{F}_{M}(K)=\operatorname{span} \delta(K) \subset \operatorname{span} \delta(M)
$$

by Theorem 3.3.7. Now assume that $m \in \operatorname{span} \delta(M)$ is of the form (3.12) and let $K=\left\{x_{1}, \ldots, x_{n}\right\}$. It is clear that $m \in \mathcal{F}_{M}(K)$, hence $\operatorname{supp}(m) \subset K$ is finite. Suppose that $\operatorname{supp}(m)=L \subsetneq K$. Then we may choose $p=x_{i} \in K \backslash L$ and pick $f \in \operatorname{Lip}_{0}(M)$ that vanishes on $L$ but satisfies $f(p)=1$ (e.g. using McShane's theorem and the fact that $p$ is at a positive distance of the closed set $L$ ). Then $\langle m, f\rangle=a_{i} \neq 0$, therefore $m \notin \mathcal{F}_{M}(L)=\mathcal{I}_{M}(L)_{\perp}$, a contradiction. We conclude that $K=\operatorname{supp}(m)$.

Note now that if $m=\sum_{n} m_{n}$ where $m_{n} \in \mathcal{F}(M)$, then it follows directly from the definition that $\operatorname{supp}(m) \subset \overline{\bigcup_{n} \operatorname{supp}(m)}$. The same happens if $m=\lim _{n} m_{n}$. In particular, by taking $m_{n} \in \operatorname{span} \delta(M)$ and using Proposition 3.3.8, we conclude that $\operatorname{supp}(m)$ is always a closed separable subset of $M$.

Let us also observe that the base point cannot be an isolated point of $\operatorname{supp}(m)$, as that would imply $m \in \mathcal{F}_{M}(K)$ where $K=\operatorname{supp}(m) \backslash\{0\}$ is closed. In particular, note that $\operatorname{supp}(0)=\varnothing$. This shows that supports are not completely stable under changes of base point, as e.g. $\operatorname{supp}(\delta(p))=\{p\}$ for $p \neq 0$ but changing the base point to $p$ converts $\delta(p)$ into 0 , with empty support. However, the discrepancy is limited to the new base point and only in the case where this point is isolated in the support.

We will now describe several equivalent characterizations of supports:

Proposition 3.3.9. Let $K$ be a closed subset of $M$, and $m \in \mathcal{F}(M)$. Then the following are equivalent:

(i) $\operatorname{supp}(m) \subset K$, 
(ii) $m \in \mathcal{F}_{M}(K)$,

(iii) $\langle m, f\rangle=\langle m, g\rangle$ for any $f, g \in \operatorname{Lip}_{0}(M)$ such that $f \uparrow_{K}=g \uparrow_{K}$.

Proof. (i) $\Rightarrow($ ii): This is an immediate consequence of Theorem 3.3.7.

(ii) $\Rightarrow(\mathrm{i})$ : This follows trivially from the definition.

(ii) $\Leftrightarrow$ (iii): Notice that (iii) is equivalent to $\langle m, f-g\rangle=0$ whenever $f-g$ vanishes in $K$, that is, to $m \in \mathcal{I}_{M}(K)_{\perp}$.

The equivalence $(\mathrm{i}) \Leftrightarrow($ iii) shows that, in particular

$$
\operatorname{supp}\left(m \circ W_{h}\right) \subset \operatorname{supp}(m) \cap \operatorname{supp}(h)
$$

for any $m \in \mathcal{F}(M)$ and $h \in \operatorname{Lip}(M)$ such that $W_{h} \in \mathcal{L}\left(\operatorname{Lip}_{0}(M)\right)$. Indeed, if $f, g \in \operatorname{Lip}_{0}(M)$ coincide on $\operatorname{supp}(m) \cap \operatorname{supp}(h)$ then $W_{h}(f)$ and $W_{h}(g)$ coincide on $\operatorname{supp}(m)$ and thus

$$
\left\langle m \circ W_{h}, f\right\rangle=\left\langle m, W_{h}(f)\right\rangle=\left\langle m, W_{h}(g)\right\rangle=\left\langle m \circ W_{h}, g\right\rangle .
$$

The inclusion in (3.13) may be strict. For instance, if $\operatorname{supp}(m)$ intersects $\operatorname{supp}(h)$ only at its boundary then $m \circ W_{h}=0$.

The following characterization of the support will be used most often, as it is usually the easier one to handle and to verify.

Proposition 3.3.10. Let $m \in \mathcal{F}(M)$ and $p \in M$. Then $p \in \operatorname{supp}(m)$ if and only if for every neighborhood $U$ of $p$ there exists a function $f \in \operatorname{Lip}_{0}(M)$ whose support is contained in $U$ and such that $\langle m, f\rangle \neq 0$. Moreover, in that case we may take $f \geq 0$.

Proof. Assume that there exists a neighborhood $U$ of $p$ such that for any function $f \in \operatorname{Lip}_{0}(M)$ with $\operatorname{supp}(f) \subset U$ we have $\langle m, f\rangle=0$. Take an open neighborhood $V$ of $p$ for which $\bar{V} \subset U$. Then $m \in \mathcal{I}_{M}(M \backslash V)_{\perp}$ because every $f \in \mathcal{I}_{M}(M \backslash V)$ satisfies $\operatorname{supp}(f) \subset \bar{V} \subset U$. Hence $m \in \mathcal{F}_{M}(M \backslash V)$, so $\operatorname{supp}(m) \subset M \backslash V$ by the definition of $\operatorname{supp}(m)$, and therefore $p \notin \operatorname{supp}(m)$.

On the other hand, suppose that $p \notin \operatorname{supp}(m)$ and let $U=M \backslash \operatorname{supp}(m)$. Then every $f \in \operatorname{Lip}_{0}(M)$ whose support is contained in $U$ obviously belongs to $\mathcal{I}_{M}(\operatorname{supp}(m))=\mathcal{F}_{M}(\operatorname{supp}(m))^{\perp}$. Therefore $\langle m, f\rangle=0$.

For the last statement, notice that $\langle m, f\rangle \neq 0$ implies that either $\left\langle m, f^{+}\right\rangle \neq 0$ or $\left\langle m, f^{-}\right\rangle \neq 0$. 
As a corollary, we obtain yet another equivalent formulation of the intersection theorem that can be interpreted as a locality principle. Loosely speaking, it says that if two elements of $\mathcal{F}(M)$ are equal locally then they are equal globally:

Corollary 3.3.11. Let $m, m^{\prime} \in \mathcal{F}(M)$. Suppose that for every $p \in M$ there is a neighborhood $U$ of $p$ such that $\langle m, f\rangle=\left\langle m^{\prime}, f\right\rangle$ for every $f \in \operatorname{Lip}_{0}(M)$ whose support is contained in $U$. Then $m=m^{\prime}$.

Proof. By assumption, for every $p \in M$ we have $\left\langle m-m^{\prime}, f\right\rangle=0$ for every $f \in$ $\operatorname{Lip}_{0}(M)$ supported in a certain neighborhood of $p$. Proposition 3.3.10 then implies that $p \notin \operatorname{supp}\left(m-m^{\prime}\right)$. Hence $\operatorname{supp}\left(m-m^{\prime}\right)=\varnothing$ and $m-m^{\prime}=0$.

Let us now collect some useful facts about positive elements of $\mathcal{F}(M)$ and their supports:

Proposition 3.3.12. Let $m, m^{\prime} \in \mathcal{F}(M)^{+}$.

(a) If $f \in \operatorname{Lip}_{0}(M), f \geq 0$ and $\langle m, f\rangle=0$, then $f=0$ on $\operatorname{supp}(m)$.

(b) If $f \in B_{\operatorname{Lip}_{0}(M)}$ and $\langle m, f\rangle=\|m\|$, then $f=\rho$ on $\operatorname{supp}(m)$.

(c) If $m \leq m^{\prime}$ then $\operatorname{supp}(m) \subset \operatorname{supp}\left(m^{\prime}\right)$.

Proof. (a) Suppose $f(p)>0$ for some $p \in \operatorname{supp}(m)$, so there are $c>0$ and $r>0$ such that $f \geq c$ in $B(p, r)$. By Proposition 3.3.10 there exists $h \in \operatorname{Lip}_{0}(M)$ such that $\operatorname{supp}(h) \subset B(p, r), h \geq 0$ and $\langle m, h\rangle>0$. Scale $h$ by a constant factor so that $h \leq c$. Then $f-h \geq 0$ but $\langle m, f-h\rangle<0$, a contradiction.

(b) Apply (a) to the function $\rho-f$.

(c) Let $p \in \operatorname{supp}(m)$ and $U$ be a neighborhood of $p$. By Proposition 3.3.10 there exists $f \in \operatorname{Lip}_{0}(M)$ such that $\operatorname{supp}(f) \subset U, f \geq 0$ and $\langle m, f\rangle>0$. But then $\left\langle m^{\prime}, f\right\rangle \geq\langle m, f\rangle>0$, so $p \in \operatorname{supp}\left(m^{\prime}\right)$ applying Proposition 3.3 .10 again.

\section{The decomposition theorem}

We finish this section with a decomposition property for supports of elements of $\mathcal{F}(M)$. This will serve as another illustration of the use of weighting operators.

Theorem 3.3.13. Let $m \in \mathcal{F}(M)$ and suppose that $\operatorname{supp}(m)=K_{1} \cup K_{2}$ where $K_{1}, K_{2}$ are closed subsets of $M$ such that $K_{1}$ is bounded and $d\left(K_{1}, K_{2}\right)>0$. Then there exists a unique decomposition $m=m_{1}+m_{2}$ where $m_{1}, m_{2} \in \mathcal{F}(M)$ are such that $\operatorname{supp}\left(m_{1}\right)=K_{1}$ and $\operatorname{supp}\left(m_{2}\right)=K_{2}$, and it satisfies

$$
\left\|m_{1}\right\| \leq\|m\| \cdot\left(2+\frac{\operatorname{rad}\left(K_{1}\right)}{d\left(K_{1}, K_{2}\right)}\right) .
$$


Proof. We start by proving uniqueness. Assume there exist decompositions $m=$ $m_{1}+m_{2}=m_{1}^{\prime}+m_{2}^{\prime}$ with $\operatorname{supp}\left(m_{1}\right), \operatorname{supp}\left(m_{1}^{\prime}\right) \subset K_{1}$ and $\operatorname{supp}\left(m_{2}\right), \operatorname{supp}\left(m_{2}^{\prime}\right) \subset$ $K_{2}$. Then $m_{1}-m_{1}^{\prime}=m_{2}^{\prime}-m_{2}$, where the left and right hand sides have their supports contained in $K_{1}$ and $K_{2}$, respectively. Therefore $\operatorname{supp}\left(m_{1}-m_{1}^{\prime}\right) \subset K_{1} \cap$ $K_{2}=\varnothing$, and so $m_{1}-m_{1}^{\prime}=0$.

Now we prove existence. Denote $D=d\left(K_{1}, K_{2}\right)$ and $R=\operatorname{rad}\left(K_{1}\right)$. Choose $\varepsilon \in\left(0, \frac{D}{2}\right)$ and let

$$
\begin{aligned}
& A=\left\{x \in M: d\left(x, K_{1}\right) \leq \varepsilon\right\}, \\
& B=\left\{x \in M: d\left(x, K_{1}\right) \geq D-\varepsilon\right\} .
\end{aligned}
$$

By McShane's theorem, there exists $h \in \operatorname{Lip}(M)$ such that $0 \leq h \leq 1, h=1$ on $A$, $h=0$ on $B$, and $\|h\|_{L} \leq d(A, B)^{-1} \leq(D-2 \varepsilon)^{-1}$. Note that $\operatorname{supp}(h)$ is bounded, with $\operatorname{rad}(\operatorname{supp}(h)) \leq R+D-\varepsilon$. Thus $m_{1}=m \circ W_{h}$ and $m_{2}=m-m_{1}=m \circ W_{1-h}$ are elements of $\mathcal{F}(M)$ and

$$
\left\|m_{1}\right\| \leq\|m\| \cdot\left(1+\frac{R+D-\varepsilon}{D-2 \varepsilon}\right)
$$

by (3.1). Notice that (3.13) yields

$$
\begin{aligned}
& \operatorname{supp}\left(m_{1}\right) \subset \operatorname{supp}(m) \cap \operatorname{supp}(h) \subset \operatorname{supp}(m) \backslash B=K_{1}, \\
& \operatorname{supp}\left(m_{2}\right) \subset \operatorname{supp}(m) \cap \operatorname{supp}(1-h) \subset \operatorname{supp}(m) \backslash A=K_{2} .
\end{aligned}
$$

and, since $m=m_{1}+m_{2}$, we have

$$
\operatorname{supp}(m) \subset \operatorname{supp}\left(m_{1}\right) \cup \operatorname{supp}\left(m_{2}\right) \subset K_{1} \cup K_{2}=\operatorname{supp}(m) .
$$

Since the unions are disjoint, all inclusions must actually be equalities. We have thus proved that the decomposition exists and satisfies (3.14). But the decomposition is unique and $\varepsilon$ was arbitrary, so taking $\varepsilon \rightarrow 0$ yields the desired bound on $\left\|m_{1}\right\|$.

It may seem that Theorem 3.3.13 is rather weak because the hypotheses that $d\left(K_{1}, K_{2}\right)>0$ and that $K_{1}$ (or $K_{2}$ ) is bounded are too strong. The following examples, inspired in [53, Example 3.24], will show that actually neither of them may be omitted from Theorem 3.3.13 in general.

Example 3.3.14. Let $M \subset c_{0}$ consist of 0 as the base point and the sequences $x_{n}=e_{n}$ and $y_{n}=\left(1+2^{-n}\right) e_{n}$, where $e_{n}$ are the standard basis vectors. Let $K_{1}$ and $K_{2}$ consist of the points $x_{n}$ and $y_{n}$, respectively. Then $K_{1}$ and $K_{2}$ are closed, bounded and disjoint, but $d\left(K_{1}, K_{2}\right)=0$. Let

$$
m=\sum_{n=1}^{\infty}\left(\delta\left(x_{n}\right)-\delta\left(y_{n}\right)\right) .
$$


Since $\left\|\delta\left(x_{n}\right)-\delta\left(y_{n}\right)\right\|=d\left(x_{n}, y_{n}\right)=2^{-n}$, the series is absolutely convergent and $m \in \mathcal{F}(M)$.

First we show that $m$ is not majorizable. Indeed, suppose that $m=m^{+}-m^{-}$ where $m^{+}, m^{-} \in \mathcal{F}(M)^{+}$. For $n \in \mathbb{N}$, define $f_{n} \in \operatorname{Lip}_{0}(M)$ such that $f_{n}\left(x_{k}\right)=1$ for $k \leq n$ and $f_{n}(x)=0$ for any other $x \in M$. Let also $h \in \operatorname{Lip}_{0}(M)$ be such that $h(x)=1$ for any $x \in M, x \neq 0$. Then $0 \leq f_{n} \leq h$ pointwise and

$$
\left\langle m^{+}, h\right\rangle \geq\left\langle m^{+}, f_{n}\right\rangle \geq\left\langle m^{+}, f_{n}\right\rangle-\left\langle m^{-}, f_{n}\right\rangle=\left\langle m, f_{n}\right\rangle=n
$$

for every $n$, which is impossible.

Now we show that $m$ cannot be expressed as $m=m_{1}+m_{2}$ where $\operatorname{supp}\left(m_{1}\right) \subset K_{1}$ and $\operatorname{supp}\left(m_{2}\right) \subset K_{2}$. Suppose there was such a decomposition. Then $m_{1} \in$ $\mathcal{F}_{M}\left(K_{1} \cup\{0\}\right)$. Since $K_{1} \cup\{0\}$ is uniformly discrete ${ }^{2}$, countable, and bounded, it is known that every element of $\mathcal{F}\left(K_{1} \cup\{0\}\right)$ can be expressed as the difference between two positive elements (indeed, each element is an $\ell_{1}$ sum of evaluation functionals, see e.g. the proof of [23, Proposition 5.1]; alternatively, use Theorem 4.4.2), hence $m_{1}=m_{1}^{+}-m_{1}^{-}$where $m_{1}^{+}, m_{1}^{-}$are positive elements of $\mathcal{F}(M)$ supported on $K_{1}$. Analogously, $m_{2}=m_{2}^{+}-m_{2}^{-}$where $m_{2}^{+}, m_{2}^{-}$are positive elements of $\mathcal{F}(M)$ supported on $K_{2}$. Therefore

$$
m=\left(m_{1}^{+}+m_{2}^{+}\right)-\left(m_{1}^{-}+m_{2}^{-}\right)
$$

is majorizable, contradicting the previous paragraph.

Example 3.3.15. Let $M \subset c_{0}$ consist of 0 as the base point and the sequences $x_{n}=2^{n} e_{n}$ and $y_{n}=\left(2^{n}+1\right) e_{n}$, where $e_{n}$ are the standard basis vectors. Let $K_{1}$ and $K_{2}$ consist of the points $x_{n}$ and $y_{n}$, respectively. Then $K_{1}$ and $K_{2}$ are closed and $d\left(K_{1}, K_{2}\right)=1$, but both are unbounded. Let

$$
m=\sum_{n=1}^{\infty} 2^{-n}\left(\delta\left(x_{n}\right)-\delta\left(y_{n}\right)\right) .
$$

Since $\left\|\delta\left(x_{n}\right)-\delta\left(y_{n}\right)\right\|=d\left(x_{n}, y_{n}\right)=1$, the series is absolutely convergent and $m \in \mathcal{F}(M)$.

Suppose that $m=m_{1}+m_{2}$ where $\operatorname{supp}\left(m_{1}\right) \subset K_{1}$ and $\operatorname{supp}\left(m_{2}\right) \subset K_{2}$. For every $n \in \mathbb{N}$ consider the function $\lambda_{n} \in \operatorname{Lip}_{0}(M)$ given by

$$
\lambda_{n}(x)= \begin{cases}d(x, 0) & \text { if } d(x, 0) \leq 2^{n} \\ 2^{n+1}-d(x, 0) & \text { if } 2^{n} \leq d(x, 0) \leq 2^{n+1} \\ 0 & \text { if } 2^{n+1} \leq d(x, 0)\end{cases}
$$

\footnotetext{
${ }^{2}$ We say that a metric space is uniformly discrete if nonzero distances are bounded below by some positive constant.
} 
Notice that $\left\|\lambda_{n}\right\|_{L} \leq 1$ and $\lambda_{n}$ converges pointwise to $\rho$, hence $\lambda_{n} \stackrel{w^{*}}{\longrightarrow} \rho$. Let $h_{n}$ be the characteristic function of $X_{n}=\left\{x_{1}, \ldots, x_{n}\right\}$, then $h_{n} \in \operatorname{Lip}_{0}(M)$ has bounded support and therefore $h_{n} \cdot \lambda_{n} \in \operatorname{Lip}_{0}(M)$. Since $\lambda_{n}$ and $h_{n} \cdot \lambda_{n}$ coincide on $K_{1}$, by Proposition 3.3.9 we have

$$
\begin{aligned}
\left\langle m_{1}, \lambda_{n}\right\rangle=\left\langle m_{1}, h_{n} \cdot \lambda_{n}\right\rangle & =\left\langle m, h_{n} \cdot \lambda_{n}\right\rangle-\left\langle m_{2}, h_{n} \cdot \lambda_{n}\right\rangle \\
& =\left\langle m, h_{n} \cdot \lambda_{n}\right\rangle=n
\end{aligned}
$$

as $\operatorname{supp}\left(m_{2}\right) \cap X_{n}=\varnothing$. Therefore $\left\langle m_{1}, \rho\right\rangle=\lim _{n}\left\langle m_{1}, \lambda_{n}\right\rangle=\infty$, a contradiction. Hence the decomposition $m=m_{1}+m_{2}$ cannot exist.

\subsection{The uniform compactification}

The rest of this chapter will be devoted to extending the notion of support to functionals in the bidual $\mathcal{F}(M)^{* *}$. As we will see, such a support cannot be defined as a subset of $M$, but rather as a subset of some topological overspace of $M$. In this section we will introduce the proper setting for such extended supports.

Recall that a compactification of a completely regular Hausdorff space, in particular of a metric space $M$, is a compact Hausdorff space that contains a dense subset that is homeomorphic to $M$ (and can thus be identified with $M$ ). Compactifications $X, Y$ of $M$ may be partially ordered by declaring that $X \leq Y$ if there is a continuous map from $Y$ onto $X$ whose restriction to $M$ is the identity; $X$ and $Y$ are then equivalent if $X \leq Y$ and $Y \leq X$. The largest compactification under this ordering is the well-known Stone-Cech compactification $\beta M$, which is characterized (up to equivalence) by the fact that any continuous function from $M$ to a compact Hausdorff space, in particular any bounded real-valued function, can be extended to a continuous function on $\beta M$. This renders it a useful tool in the study of spaces of continuous functions. In particular, it is used for the study of Lipschitz spaces in [53], and we will also make use of it throughout Chapter 5 as a tool in the study of the extreme points of $B_{\mathcal{F}(M)}$.

When dealing with metric spaces and Lipschitz functions, however, the common Stone-Čech compactification carries an important drawback for some of our intended purposes, namely the fact that Lipschitz functions do not necessarily separate points of $\beta M$. Let us see a simple example of how this can happen:

Example 3.4.1. Let $M \subset \mathbb{R}$ consist of 0 and the points $x_{n}=n$ and $y_{n}=n+2^{-n}$ for $n \in \mathbb{N}$. By compactness there is a subnet $\left(x_{n_{i}}, y_{n_{i}}\right)$ of the sequence $\left(x_{n}, y_{n}\right)$ such that $x_{n_{i}}$ and $y_{n_{i}}$ converge to points $\xi$ and $\eta$ of $\beta M$, respectively. Since $M$ is topologically discrete, any function on $M$ is continuous and we may take for instance $f \in C(M)$ such that $f\left(x_{n}\right)=0$ and $f\left(y_{n}\right)=1$ for every $n$. Then $f(\xi)=0$ and $f(\eta)=1$, hence $\xi \neq \eta$. However $\xi$ and $\eta$ cannot be separated by Lipschitz functions: indeed, if $f \in \operatorname{Lip}(M)$ then $\left|f\left(x_{n}\right)-f\left(y_{n}\right)\right| \leq 2^{-n}\|f\|_{L}$, and taking limits yields $f(\xi)=f(\eta)$. 
In fact, according to [55, Theorem 3.4] Lipschitz functions separate points of $\beta M$ if and only if there is a compact subset $K$ of $M$ such that $M \backslash U$ is uniformly discrete for every open set $U \supset K$; recall that a metric space is uniformly discrete if the distance between different points is bounded below by a positive constant.

Instead of $\beta M$, we will be using the uniform or Samuel compactification of $M$, and denote it as $M^{\mathcal{U}}$. The suggested reference for information about this object is [55]. The following statement collects its defining properties:

Proposition 3.4.2 ([55, Corollary 2.4]). Let $M$ be a metric space. Then there exists a compactification $M^{\mathcal{U}}$ of $M$ with the following properties:

(i) Every bounded, uniformly continuous function $f: M \rightarrow \mathbb{R}$ can be extended uniquely to a continuous function $f^{\mathcal{U}}: M^{\mathcal{U}} \rightarrow \mathbb{R}$.

(ii) Given two subsets $A, B \subset M$, their closures in $M^{\mathcal{U}}$ are disjoint if and only if $d(A, B)>0$.

Moreover, these properties determine $M^{\mathcal{U}}$ uniquely up to equivalence.

We will denote the closure of $A \subset M$ in $M^{\mathcal{U}}$ by $\bar{A}^{\mathcal{U}}$. Note that $\bar{A}^{\mathcal{U}}$ and $A^{\mathcal{U}}$ are equivalent compactifications of the metric space $A$ by [55, Theorem 2.9], so this notation shall lead to no confusion. We will also need the following converse to property (i):

Proposition 3.4.3 ([55, Theorem 2.5]). If $f: M \rightarrow \mathbb{R}$ is bounded and can be extended continuously to $M^{\mathcal{U}}$, then $f$ is uniformly continuous.

Combining property (ii) with the usual separation axioms immediately yields the following metric separation property, that we will be using repeatedly:

Proposition 3.4.4. Let $K, L$ be disjoint closed subsets of $M^{\mathcal{U}}$. Then there are disjoint open neighborhoods $V, W$ of $K, L$ such that $d(V \cap M, W \cap M)>0$.

Thus disjoint closed subsets of $M^{\mathcal{U}}$ can be separated by (extensions of) Lipschitz functions on $M$. In particular, Lipschitz functions separate points of $M^{\mathcal{U}}$ as required. In fact, $M^{\mathcal{U}}$ may be identified with the quotient space of $\beta M$ obtained by identifying those points that cannot be separated by Lipschitz functions.

Property (i) only covers the extension of bounded uniformly continuous (in particular, Lipschitz) functions on $M$, but in general we may need to deal with extensions of unbounded functions, too. It is proved (for a more general setting) in [25, Proposition 1.4] that unbounded Lipschitz functions can be continuously extended to $M^{\mathcal{U}}$ if we enlarge the range from $\mathbb{R}$ to its one-point compactification $\mathbb{R} \cup\{\infty\}$; see also Section 1 of [26]. The following version of that property will be more useful for our purposes: 
Proposition 3.4.5. Every Lipschitz function $f: M \rightarrow \mathbb{R}$ can be extended uniquely to a continuous function $f^{\mathcal{U}}: M^{\mathcal{U}} \rightarrow[-\infty,+\infty]$.

Indeed, this follows easily using (ii) from either the $\mathbb{R} \cup\{\infty\}$ version or, more directly, from the Taimanov extension theorem (see e.g. [21, Theorem 3.2.1]).

\section{The Lipschitz realcompactification}

Let us consider the elements $\zeta \in M^{\mathcal{U}}$ that satisfy any of the following clearly equivalent conditions:

- $\zeta$ is the limit of a bounded net in $M$,

- $\rho^{\mathcal{U}}(\zeta)<\infty$ (for any choice of base point in $M$ ),

- $\left|f^{\mathcal{U}}(\zeta)\right|<\infty$ for all $f \in \operatorname{Lip}(M)$.

The set $M^{\mathcal{R}}$ of all such elements is called the Lipschitz realcompactification of $M$ in [26]. In general we have $M \subset M^{\mathcal{R}} \subset M^{\mathcal{U}}$, and it should be clear that $M^{\mathcal{R}}=M^{\mathcal{U}}$ if and only if $M$ is bounded, and $M^{\mathcal{R}}=M$ if and only if $M$ is a HeineBorel space. Recall that a metric space is Heine-Borel (alternatively called proper or boundedly compact) if all its closed balls are compact; in particular, compact spaces are Heine-Borel.

Notice also that the evaluation functional $\delta(\zeta): f \mapsto f^{\mathcal{U}}(\zeta)$ is an element of $\mathcal{F}(M)^{* *}$ if and only if $\zeta \in M^{\mathcal{R}}$, and its norm is $\|\delta(\zeta)\|=\rho^{\mathcal{U}}(\zeta)$. Let us also mention that $(f g)^{\mathcal{U}}(\zeta)=f^{\mathcal{U}}(\zeta) g^{\mathcal{U}}(\zeta)$ for any $\zeta \in M^{\mathcal{R}}$ and $f, g \in \operatorname{Lip}(M)$ such that $f g \in \operatorname{Lip}(M)$; this is not valid for $\zeta \notin M^{\mathcal{R}}$, as an indeterminate limit of type $0 \times \infty$ may appear.

\section{Derivations}

Let us now introduce a class of functionals on $\operatorname{Lip}_{0}(M)$ that will play a role in what follows. In [53, Section 7.5], a derivation at a point $x \in M$ (or $M^{\mathcal{U}}$, more generally) is defined as an element $\phi \in \mathcal{F}(M)^{* *}$ that satisfies the relation

$$
\langle f g, \phi\rangle=\langle f, \phi\rangle \cdot g(x)+f(x) \cdot\langle g, \phi\rangle
$$

for any $f, g \in \operatorname{Lip}_{0}(M)$. In general $f g$ is not a Lipschitz function, and this is likely one of the reasons why the original definition is only given for bounded $M$; another one is that $f(x)$ may not be finite if $x \notin M^{\mathcal{R}}$, which is possible when $M$ is unbounded. In order to eliminate these restrictions and extend the domain of the definition, we prefer to use the following alternative formulation:

Definition 3.4.6. Let $\phi \in \mathcal{F}(M)^{* *}$ and $\zeta \in M^{\mathcal{U}}$. We say that $\phi$ is a derivation at $\zeta$ if $\langle f, \phi\rangle=0$ for any $f \in \operatorname{Lip}_{0}(M)$ such that $f^{\mathcal{U}}$ is constant in a neighborhood of $\zeta$. 
Lemma 7.47 in [53] asserts that both definitions are equivalent in the original setting, i.e. when $M$ is bounded, and it follows easily that they are also equivalent in general for $\zeta \in M^{\mathcal{R}}$. This formulation makes it obvious that nontrivial derivations at $x \in M$ can only exist if $x$ is not an isolated point. Also, derivations at $\zeta \in M^{\mathcal{R}} \backslash\{0\}$ can never be positive, since given $f \in \operatorname{Lip}_{0}(M)$ it is easy to construct $g \in \operatorname{Lip}_{0}(M)^{+}$such that $g-f$ is constant in a neighborhood of $\zeta$. On the other hand, there are always positive derivations at 0 (assuming it is not isolated): indeed, let $x_{n} \rightarrow 0$ and $m_{n}=\delta\left(x_{n}\right) / d\left(x_{n}, 0\right) \in B_{\mathcal{F}(M)}$, then $\left(m_{n}\right)$ must have a subnet that converges weak* to $\phi \in \mathcal{F}(M)^{* *}$. This $\phi$ is clearly a positive derivation at 0 such that $\|\phi\|=\langle\rho, \phi\rangle=1$.

It is interesting to note that derivations at different points of $M^{\mathcal{R}}$ are "orthogonal" to each other and to weak* continuous functionals in the following sense.

Proposition 3.4.7. Let $m \in \mathcal{F}(M)$, and let $\left(\phi_{n}\right) \subset \mathcal{F}(M)^{* *}$ be a sequence of derivations at different points of $M^{\mathcal{R}}$ such that $\sum\left\|\phi_{n}\right\|<\infty$. Then

$$
\left\|m+\sum_{n=1}^{\infty} \phi_{n}\right\|=\|m\|+\sum_{n=1}^{\infty}\left\|\phi_{n}\right\| .
$$

Proof. It is clearly enough to prove the theorem for a finite sum $m+\phi_{1}+\ldots+\phi_{n}$, and we may assume that each $\phi_{i}$ is nonzero. Fix $\varepsilon>0$ and choose $f, g_{i} \in S_{\operatorname{Lip}_{0}(M)}$ such that $\langle m, f\rangle=\|m\|$ and $\left\langle g_{i}, \phi_{i}\right\rangle>\left\|\phi_{i}\right\|-\frac{\varepsilon}{n}$ for $i=1, \ldots, n$. Suppose that $\phi_{i}$ is a derivation at $\zeta_{i} \in M^{\mathcal{R}}$ and let $A$ be the set of those $\zeta_{i}$ that belong to $M$. Let $\mathfrak{F}$ be the family of all finite subsets of $M \backslash(A \cup\{0\})$, directed by inclusion. We will construct a net $\left(h_{E}\right)_{E \in \mathfrak{F}}$ in $\operatorname{Lip}_{0}(M)$ such that $\left\|h_{E}\right\|_{L} \leq 1+4 \varepsilon$ for every $E \in \mathfrak{F}$.

Fix $E \in \mathfrak{F}$. Since the $\zeta_{i}$ are all different from each other and not contained in $E$, we may find disjoint open neighborhoods $U_{i}$ of $\zeta_{i}$ such that the sets $U_{i} \cap M$ are at a positive distance from each other and from $E$; also, if $\zeta_{i} \neq 0$ then we also assume that $d\left(U_{i} \cap M, 0\right)>0$. Let $r>0$ be smaller than all of those distances. For each $i=1, \ldots, n$ let

$$
V_{i}=\left\{\xi \in M^{\mathcal{U}}:\left|g_{i}^{\mathcal{U}}(\xi)-g_{i}^{\mathcal{U}}\left(\zeta_{i}\right)\right|<\varepsilon r \text { and }\left|f^{\mathcal{U}}(\xi)-f^{\mathcal{U}}\left(\zeta_{i}\right)\right|<\varepsilon r\right\}
$$

which is clearly an open neighborhood of $\zeta_{i}$, and let $W_{i}=U_{i} \cap V_{i}$. Define the function $h_{E}$ on the set $\{0\} \cup E \cup \bigcup_{i=1}^{n}\left(W_{i} \cap M\right)$ by

$$
h_{E}(x)= \begin{cases}f(x) & , x \in E \cup\{0\} \\ g_{i}(x)-g_{i}^{\mathcal{U}}\left(\zeta_{i}\right)+f^{\mathcal{U}}\left(\zeta_{i}\right) & , x \in W_{i} \cap M\end{cases}
$$

for $i=1, \ldots, n$. Notice that if $\zeta_{i}=0$ for some $i$ then both cases yield the same value $h_{E}(0)=0$.

Now let us estimate $\left\|h_{E}\right\|_{L}$. It is clear that $\left|h_{E}(x)-h_{E}(y)\right| \leq d(x, y)$ if $x, y$ belong to $E \cup\{0\}$ or to $W_{i} \cap M$ for the same $i$. If $x \in W_{i} \cap M$ and $y \in W_{j} \cap M$ for $i \neq j$ 
then $d(x, y) \geq r$ and

$$
\begin{aligned}
\left|h_{E}(x)-h_{E}(y)\right| & \leq\left|g_{i}(x)-g_{i}^{\mathcal{U}}\left(\zeta_{i}\right)\right|+\left|g_{j}(y)-g_{j}^{\mathcal{U}}\left(\zeta_{j}\right)\right|+\left|f^{\mathcal{U}}\left(\zeta_{i}\right)-f^{\mathcal{U}}\left(\zeta_{j}\right)\right| \\
& \leq 2 \varepsilon r+\left|f^{\mathcal{U}}\left(\zeta_{i}\right)-f(x)\right|+\left|f^{\mathcal{U}}\left(\zeta_{j}\right)-f(y)\right|+|f(x)-f(y)| \\
& \leq 4 \varepsilon r+d(x, y) \\
& \leq(1+4 \varepsilon) d(x, y)
\end{aligned}
$$

Otherwise, if $x \in W_{i} \cap M$ and $y \in E$ then $d(x, y) \geq r$ again and

$$
\begin{aligned}
\left|h_{E}(x)-h_{E}(y)\right| & \leq\left|g_{i}(x)-g_{i}^{\mathcal{U}}\left(\zeta_{i}\right)\right|+\left|f^{\mathcal{U}}\left(\zeta_{i}\right)-f(y)\right| \\
& \leq \varepsilon r+\left|f^{\mathcal{U}}\left(\zeta_{i}\right)-f(x)\right|+|f(x)-f(y)| \\
& \leq 2 \varepsilon r+d(x, y) \\
& \leq(1+2 \varepsilon) d(x, y) .
\end{aligned}
$$

So $\left\|h_{E}\right\|_{L} \leq 1+4 \varepsilon$ as claimed. Finally, extend $h_{E}$ to $M$ using McShane's theorem.

We have thus built a bounded net $\left(h_{E}\right)_{E \in \mathfrak{F}}$ in $\operatorname{Lip}_{0}(M)$. Now notice that $h_{E}$ converges pointwise to $f$. Indeed, $h_{E}(x)=f(x)$ for all $x \in A \cup\{0\}$ and all $E \in \mathfrak{F}$ by construction, and $h_{E}(x)=f(x)$ for any $x \in M \backslash(A \cup\{0\})$ whenever $E \supset\{x\}$. Thus $\left(h_{E}\right)$ converges weak* to $f$, and therefore we can choose $E \subset \mathfrak{F}$ such that $\left\langle m, h_{E}\right\rangle>\langle m, f\rangle-\varepsilon$. By construction, $h_{E}-g_{i}$ is constant in a neighborhood of each $\zeta_{i}$, therefore $\left\langle h_{E}, \phi_{i}\right\rangle=\left\langle g_{i}, \phi_{i}\right\rangle$. Putting it all together, we have

$$
\left\langle h_{E}, m+\sum_{i=1}^{n} \phi_{i}\right\rangle>\langle m, f\rangle-\varepsilon+\sum_{i=1}^{n}\left\langle g_{i}, \phi_{i}\right\rangle>\|m\|+\sum_{i=1}^{n}\left\|\phi_{i}\right\|-2 \varepsilon
$$

and hence

$$
\left\|m+\sum_{i=1}^{n} \phi_{i}\right\|>\frac{\|m\|+\sum_{i=1}^{n}\left\|\phi_{i}\right\|-2 \varepsilon}{\left\|h_{E}\right\|_{L}} \geq \frac{\|m\|+\sum_{i=1}^{n}\left\|\phi_{i}\right\|-2 \varepsilon}{1+4 \varepsilon} .
$$

Letting $\varepsilon \rightarrow 0$ yields the desired result.

\subsection{Supports in $\mathcal{F}(M)^{* *}$}

In this section we will propose a generalization of the concept of support for elements of $\mathcal{F}(M)$ that is applicable to functionals in its bidual. Unfortunately, some of its properties break down for functionals with content that "lies at infinity". In order to make this statement more precise, we will first analyze the structure of $\mathcal{F}(M)^{* *}$ and establish a decomposition of general functionals into elements that concentrate at different domains. 


\section{Structure of $\mathcal{F}(M)^{* *}$}

Before continuing, let us make the following observation: for any $\phi \in \mathcal{F}(M)^{* *}$, the sequence $\left(\phi \circ W_{H_{n}}\right)$ is Cauchy. Indeed, for $m>n \in \mathbb{N}$ we have that

$$
\left\|\phi \circ W_{H_{m}}-\phi \circ W_{H_{n}}\right\|=\left\|\sum_{k=n+1}^{m} \phi \circ W_{\Lambda_{k}}\right\| \leq \sum_{k=n+1}^{\infty}\left\|\phi \circ W_{\Lambda_{k}}\right\|
$$

can be made arbitrarily small by (3.7). So $\left(\phi \circ W_{H_{n}}\right)$ converges in norm to a functional in $\mathcal{F}(M)^{* *}$ that can be interpreted as "the part of $\phi$ that is concentrated away from infinity", and since $\phi \circ W_{G_{n}}=\phi-\phi \circ W_{H_{n}}$, the limit of $\left(\phi \circ W_{G_{n}}\right)$ also exists and can be thought of as "the part of $\phi$ that lies at infinity". Analogously, $\left(\phi \circ W_{G_{-n}}\right)$ and $\left(\phi \circ W_{H_{-n}}\right)$ converge in norm and the limits can be understood as "the part of $\phi$ that is concentrated away from the base point" and "the part that lies at the base point", respectively.

With this idea in mind, let us introduce some terminology for the following classes of functionals:

Definition 3.5.1. Let $\phi \in \mathcal{F}(M)^{* *}$. We say that $\phi$

- is concentrated at infinity if $\phi \circ W_{H_{n}}=0$ for all $n \in \mathbb{N}$,

- avoids infinity if $\lim _{n} \phi \circ W_{H_{n}}=\phi$,

- avoids infinity strongly if $\phi \circ W_{H_{n}}=\phi$ for some $n \in \mathbb{N}$,

- is concentrated at 0 if $\phi \circ W_{G_{-n}}=0$ for all $n \in \mathbb{N}$,

- avoids 0 if $\lim _{n} \phi \circ W_{G_{-n}}=\phi$,

- avoids 0 strongly if $\phi \circ W_{G_{-n}}=\phi$ for some $n \in \mathbb{N}$.

Let us mention some easy facts about these classes. If $\phi \in \mathcal{F}(M)^{* *}$ avoids 0 (resp. infinity) strongly then it avoids 0 (resp. infinity), and if $\phi$ is concentrated at infinity then it avoids 0 strongly and vice versa. Moreover, since $W_{H_{n}}(f)$ and $W_{G_{-n}}(f)$ converge weak* to $f$ for every $f \in \operatorname{Lip}_{0}(M)$, it follows from the remark before Definition 3.5.1 that every element of $\mathcal{F}(M)$ avoids 0 and infinity. It is also not difficult to see that $\phi$ avoids 0 and infinity strongly if and only if $\phi \circ W_{\Pi_{n}}=\phi$ for some $n$ and it avoids 0 and infinity if and only if $\lim _{n} \phi \circ W_{\Pi_{n}}=\phi$.

Note also that if 0 is an isolated point of $M$ then every functional $\phi \in \mathcal{F}(M)^{* *}$ avoids 0 strongly, and if $M$ is bounded then every $\phi$ avoids infinity strongly. In fact, those functionals that avoid infinity strongly can be completely identified

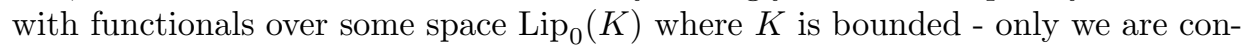
sidering them as elements of $\operatorname{Lip}_{0}(M)^{*}$ for some possibly unbounded overspace $M$ of $K$. Similarly, functionals that avoid 0 strongly can be identified with functionals over some space $\operatorname{Lip}_{0}(K)$ where $K$ has an isolated base point. In view of Proposition 3.3.9, it is also easy to see that an element of $\mathcal{F}(M)$ avoids infinity 
strongly precisely when its support is bounded. This is not the case in $\mathcal{F}(M)^{* *}$, as we shall see with Example 3.5.15.

While the definitions above are given in terms of the auxiliary functions $H_{n}$ and $G_{n}$ defined in (3.2) and (3.3), it is possible to express some of these notions equivalently in a much more general way. The following proposition contains some such characterizations; additional ones will be given in Proposition 3.5.18.

Proposition 3.5.2. Let $\phi \in \mathcal{F}(M)^{* *}$.

(a) $\phi$ is concentrated at infinity if and only if $\langle f, \phi\rangle=0$ for every $f \in \operatorname{Lip}_{0}(M)$ with bounded support.

(b) $\phi$ is concentrated at 0 if and only if it is a derivation at 0.

(c) $\phi$ avoids infinity strongly if and only if $\phi=\phi \circ W_{h}$ for some $h \in \operatorname{Lip}(M)$ with bounded support.

Proof. (a) Suppose that $\phi$ is concentrated at infinity, and pick $f \in \operatorname{Lip}_{0}(M)$ with bounded support. Choose $n \in \mathbb{N}$ such that $2^{n}>\operatorname{rad}(\operatorname{supp}(f))$. Then $f=f H_{n}$ and hence

$$
\langle f, \phi\rangle=\left\langle f H_{n}, \phi\right\rangle=\left\langle f, \phi \circ W_{H_{n}}\right\rangle=0 .
$$

For the converse implication, if $f \in \operatorname{Lip}_{0}(M)$ and $n \in \mathbb{N}$ then $f H_{n} \in \operatorname{Lip}_{0}(M)$ has bounded support, hence $\left\langle f, \phi \circ W_{H_{n}}\right\rangle=\left\langle f H_{n}, \phi\right\rangle=0$. Therefore $\phi \circ W_{H_{n}}=0$.

(b) If $\phi$ is a derivation at 0 , then for every $n \in \mathbb{N}$ and $f \in \operatorname{Lip}_{0}(M)$ we have $\left\langle f, \phi \circ W_{G_{-n}}\right\rangle=\left\langle f G_{-n}, \phi\right\rangle=0$ since $f G_{-n}=0$ in a neighborhood of 0 , so $\phi$ is concentrated at 0 . Conversely, suppose that $\phi$ is concentrated at 0 and let $f \in \operatorname{Lip}_{0}(M)$ be constant, i.e. 0 , in a neighborhood of 0 . Then $f=f G_{-n}$ for $n$ large enough and

$$
\langle f, \phi\rangle=\left\langle f G_{-n}, \phi\right\rangle=\left\langle f, \phi \circ W_{G_{-n}}\right\rangle=0 .
$$

Hence $\phi$ is a derivation at 0 .

(c) One implication is obvious taking $h=H_{n}$. For the converse, let $n$ be such that $2^{n}>\operatorname{rad}(\operatorname{supp}(h))$, then $h H_{n}=h$ and hence

$$
\phi \circ W_{H_{n}}=\left(\phi \circ W_{h}\right) \circ W_{H_{n}}=\phi \circ W_{h H_{n}}=\phi \circ W_{h}=\phi .
$$

Every functional in $\mathcal{F}(M)^{* *}$ can be canonically decomposed as a sum of elements of the classes introduced in Definition 3.5.1 as follows:

Theorem 3.5.3. Every $\phi \in \mathcal{F}(M)^{* *}$ can be expressed as

$$
\phi=\phi_{0}+\phi_{s}+\phi_{\infty}
$$


where $\phi_{0}$ is a derivation at $0, \phi_{\infty}$ is concentrated at infinity, and $\phi_{s}$ avoids 0 and infinity. This expression is unique and

$$
\begin{aligned}
\phi_{0} & =\lim _{n \rightarrow \infty} \phi \circ W_{H_{-n}} \\
\phi_{s} & =\lim _{n \rightarrow \infty} \phi \circ W_{\Pi_{n}} . \\
\phi_{\infty} & =\lim _{n \rightarrow \infty} \phi \circ W_{G_{n}}
\end{aligned}
$$

Moreover we have

$$
\|\phi\|=\left\|\phi_{0}\right\|+\left\|\phi_{s}\right\|+\left\|\phi_{\infty}\right\| .
$$

If $\phi$ is positive then so are $\phi_{0}, \phi_{s}$ and $\phi_{\infty}$.

Proof. Let us first prove existence of the decomposition. Let $\phi_{0}, \phi_{s}, \phi_{\infty}$ be given by (3.16). We have already seen that all of these limits exist, and (3.15) follows from taking limits in the identity

$$
\phi=\phi \circ W_{H_{-(n+1)}}+\phi \circ W_{\Pi_{n}}+\phi \circ W_{G_{n}} .
$$

Notice that, for any fixed $n \geq 1$

$$
\phi_{0} \circ W_{G_{-n}}=\left(\lim _{k \rightarrow \infty} \phi \circ W_{H_{-k}}\right) \circ W_{G_{-n}}=\lim _{k \rightarrow \infty} \phi \circ W_{H_{-k} G_{-n}}=0
$$

as $H_{-k} G_{-n}=0$ eventually, so $\phi_{0}$ is concentrated at 0 , hence it is a derivation at 0 by Proposition 3.5.2(b). By an analogous argument, $\phi_{\infty}$ is concentrated at infinity. We also have

$$
\begin{aligned}
\phi_{s} \circ W_{H_{n}} & =\left(\lim _{k \rightarrow \infty} \phi \circ W_{\Pi_{k-1}}\right) \circ W_{H_{n}} \\
& =\left(\lim _{k \rightarrow \infty} \phi \circ W_{G_{-k} H_{k-1}}\right) \circ W_{H_{n}}=\lim _{k \rightarrow \infty} \phi \circ W_{G_{-k} H_{n}}
\end{aligned}
$$

and therefore

$$
\lim _{n \rightarrow \infty} \phi_{s} \circ W_{H_{n}}=\lim _{n \rightarrow \infty} \lim _{k \rightarrow \infty} \phi \circ W_{G_{-k} H_{n}}=\lim _{n \rightarrow \infty} \lim _{k \rightarrow \infty} \sum_{i=-k+1}^{n} \phi \circ W_{\Lambda_{i}}=\phi_{s}
$$

because the series (3.6) converges absolutely by (3.7). That is, $\phi_{s}$ avoids infinity. A similar argument shows that it also avoids 0 . The statement about positivity follows from the fact that all weighting operators used in the construction preserve positivity.

Next we prove that the decomposition is unique. Assume that

$$
\phi=\phi_{0}+\phi_{s}+\phi_{\infty}=\phi_{0}^{\prime}+\phi_{s}^{\prime}+\phi_{\infty}^{\prime}
$$


where $\phi_{0}, \phi_{0}^{\prime}$ are concentrated at $0, \phi_{\infty}, \phi_{\infty}^{\prime}$ are concentrated at infinity, and $\phi_{s}, \phi_{s}^{\prime}$ avoid 0 and infinity. Then $\phi_{0}+\phi_{s}-\phi_{0}^{\prime}-\phi_{s}^{\prime}=\phi_{\infty}^{\prime}-\phi_{\infty}$, and we have

$$
\begin{aligned}
0=\lim _{n \rightarrow \infty}\left(\phi_{\infty}^{\prime}-\phi_{\infty}\right) \circ W_{H_{n}} & =\lim _{n \rightarrow \infty}\left(\phi_{0}-\phi_{0}^{\prime}+\phi_{s}-\phi_{s}^{\prime}\right) \circ W_{H_{n}} \\
& =\phi_{0}-\phi_{0}^{\prime}+\lim _{n \rightarrow \infty}\left(\phi_{s}-\phi_{s}^{\prime}\right) \circ W_{H_{n}} \\
& =\phi_{0}-\phi_{0}^{\prime}+\phi_{s}-\phi_{s}^{\prime}
\end{aligned}
$$

so that $\phi_{0}^{\prime}-\phi_{0}=\phi_{s}-\phi_{s}^{\prime}$. It follows similarly that

$$
0=\lim _{n \rightarrow \infty}\left(\phi_{0}^{\prime}-\phi_{0}\right) \circ W_{G_{-n}}=\lim _{n \rightarrow \infty}\left(\phi_{s}-\phi_{s}^{\prime}\right) \circ W_{G_{-n}}=\phi_{s}-\phi_{s}^{\prime} .
$$

Hence $\phi_{s}=\phi_{s}^{\prime}$, and uniqueness follows.

Finally, we will prove (3.17). Fix $\varepsilon>0$, and choose functions $f_{0}, f_{s}, f_{\infty} \in S_{\operatorname{Lip}_{0}(M)}$ such that $\left\langle f_{0}, \phi_{0}\right\rangle>\left\|\phi_{0}\right\|-\varepsilon,\left\langle f_{s}, \phi_{s}\right\rangle>\left\|\phi_{s}\right\|-\varepsilon$ and $\left\langle f_{\infty}, \phi_{\infty}\right\rangle>\left\|\phi_{\infty}\right\|-\varepsilon$. By the convergence of (3.7), we may find $n \in \mathbb{N}$ such that

$$
\sum_{\substack{k \in \mathbb{Z} \\|k|>n}}\left\|\phi \circ W_{\Lambda_{k}}\right\|<\varepsilon
$$

This implies that $\left\|\phi_{s}-\phi \circ W_{\Pi_{n}}\right\|<\varepsilon$ and, more generally

$$
\left\|\phi \circ W_{1-H_{-m}-\Pi_{n}-G_{m}}\right\|=\left\|\sum_{k=-m+1}^{-(n+1)} \phi \circ W_{\Lambda_{k}}+\sum_{k=n+1}^{m} \phi \circ W_{\Lambda_{k}}\right\|<\varepsilon
$$

for any $m>n$. Fix $m>n+2$, to be determined later. Since $\phi \circ W_{H_{-m}} \rightarrow \phi_{0}$ and $\phi \circ W_{G_{m}} \rightarrow \phi_{\infty}$ as $m \rightarrow \infty, m$ can be chosen to be large enough that $\left\|\phi_{0}-\phi \circ W_{H_{-m}}\right\|<\varepsilon$ and $\left\|\phi_{\infty}-\phi \circ W_{G_{m}}\right\|<\varepsilon$.

Let $M^{\prime}=M_{0} \cup M_{s} \cup M_{\infty}$ where

$$
\begin{aligned}
M_{0} & =B\left(0,2^{-m+1}\right) \\
M_{s} & =B\left(0,2^{n+1}\right) \backslash B\left(0,2^{-n-1}\right) \\
M_{\infty} & =M \backslash B\left(0,2^{m}\right)
\end{aligned}
$$

and define $g: M^{\prime} \rightarrow \mathbb{R}$ by $g=f_{0}$ on $M_{0}, g=f_{s}$ on $M_{s}$ and $g=f_{\infty}$ on $M_{\infty}$. Let us estimate $\|g\|_{L}$. Clearly $|g(x)-g(y)| \leq d(x, y)$ when $x, y$ belong to the same one of the disjoint sets $M_{0}, M_{s}, M_{\infty}$. Otherwise suppose $d(x, 0)>d(y, 0)$, then we actually have $d(x, 0) \geq 2^{m-n-2} d(y, 0)$ and hence

$$
\frac{|g(x)-g(y)|}{d(x, y)} \leq \frac{|g(x)|+|g(y)|}{d(x, y)} \leq \frac{d(x, 0)+d(y, 0)}{d(x, 0)-d(y, 0)} \leq \frac{2^{m-n-2}+1}{2^{m-n-2}-1} .
$$


Thus, by choosing $m$ large enough we can guarantee that $\|g\|_{L}<1+\varepsilon$. Now extend $g$ to $M$ without increasing its norm. Then we have

$$
\begin{aligned}
\langle g, \phi\rangle=\left\langle g, \phi \circ W_{H_{-m}}\right\rangle+ & \left\langle g, \phi \circ W_{\Pi_{n}}\right\rangle \\
& +\left\langle g, \phi \circ W_{G_{m}}\right\rangle+\left\langle g, \phi \circ W_{1-H_{-m}-\Pi_{n}-G_{m}}\right\rangle .
\end{aligned}
$$

Notice that

$$
\left\langle g, \phi \circ W_{H_{-m}}\right\rangle=\left\langle f_{0}, \phi \circ W_{H_{-m}}\right\rangle=\left\langle f_{0}, \phi_{0}\right\rangle-\left\langle f_{0}, \phi_{0}-\phi \circ W_{H_{-m}}\right\rangle>\left\|\phi_{0}\right\|-2 \varepsilon
$$

and similarly the second and third terms in (3.19) are bounded below by $\left\|\phi_{s}\right\|-2 \varepsilon$ and $\left\|\phi_{\infty}\right\|-2 \varepsilon$, respectively. Substituting into (3.19) and using (3.18) we obtain

$$
\langle g, \phi\rangle>\left\|\phi_{0}\right\|+\left\|\phi_{s}\right\|+\left\|\phi_{\infty}\right\|-6 \varepsilon-\|g\|_{L} \varepsilon
$$

and it follows that

$$
\|\phi\| \geq \frac{\langle g, \phi\rangle}{\|g\|_{L}}>\frac{\left\|\phi_{0}\right\|+\left\|\phi_{s}\right\|+\left\|\phi_{\infty}\right\|-6 \varepsilon}{1+\varepsilon}-\varepsilon .
$$

Letting $\varepsilon \rightarrow 0$ yields $\|\phi\| \geq\left\|\phi_{0}\right\|+\left\|\phi_{s}\right\|+\left\|\phi_{\infty}\right\|$, and the converse inequality is obvious.

By adding $\phi_{A}=\phi_{0}+\phi_{s}$ (or using similar reasoning), we obtain a similar decomposition of $\phi$ into a part that is concentrated at infinity and a part that avoids infinity, and they do not depend on the choice of base point:

Corollary 3.5.4. Every $\phi \in \mathcal{F}(M)^{* *}$ can be expressed uniquely as $\phi=\phi_{A}+\phi_{\infty}$ where $\phi_{A}$ avoids infinity and $\phi_{\infty}$ is concentrated at infinity. These terms are given by $\phi_{A}=\lim _{n} \phi \circ W_{H_{n}}$ and $\phi_{\infty}=\lim _{n} \phi \circ W_{G_{n}}$. Moreover we have $\|\phi\|=$ $\left\|\phi_{A}\right\|+\left\|\phi_{\infty}\right\|$.

\section{Extended supports}

Let us now deal with the generalized concept of support for elements of $\mathcal{F}(M)^{* *}$. Before giving the definition, we will briefly discuss the reasoning behind the changes with respect to the previous notion for $\mathcal{F}(M)$.

First of all, for any such notion it is a reasonable expectation that the support of an evaluation functional is precisely the point where the evaluation takes place. That is the case for the support of elements $\delta(x) \in \mathcal{F}(M)$ for $x \in M$, by Proposition 3.3.8. However, we have seen that the evaluation on any $\zeta \in M^{\mathcal{R}} \backslash M$ is also an element of $\mathcal{F}(M)^{* *}$. In order to accommodate for that, the support should now be a subset of some compactification of $M$. And since any compactification larger than $M^{\mathcal{U}}$ could contain elements that are not separated by Lipschitz functions 
and therefore lead to inconsistencies when defining the support of their associated evaluation functionals, the appropriate choice would be precisely $M^{\mathcal{U}}$.

Second, it is not possible to obtain a support that is sharp in the sense of Proposition 3.3.9, in that the action of $\phi \in \mathcal{F}(M)^{* *}$ on a function $f \in \operatorname{Lip}_{0}(M)$ only depends on the values it (or its extension $f^{\mathcal{U}}$ ) takes on the support of $\phi$. The best example to illustrate this issue are derivations. For instance, let $\phi$ be the derivation at 0 that we have constructed just after Definition 3.4.6. It does not make sense for the support of $\phi$ to contain any point other than 0 , since the value of $\langle f, \phi\rangle$ is independent of the behavior of $f$ away from 0 , so the support should be just $\{0\}$. However, note that $\langle f, \phi\rangle$ does not depend just on the value of $f$ at 0 , it depends on its behavior in a neighborhood of 0 (any neighborhood is valid).

The previous discussion suggests that the support of a functional $\phi \in \mathcal{F}(M)^{* *}$ should be defined as a subset $S$ of $M^{\mathcal{U}}$ that satisfies the following property, weaker than Proposition 3.3.9: $\langle f, \phi\rangle=\langle g, \phi\rangle$ whenever $f^{\mathcal{U}}$ coincides with $g^{\mathcal{U}}$ on some open set containing $S$. This motivates the next definition:

Definition 3.5.5. Let $\phi \in \mathcal{F}(M)^{* *}$. The set

$$
\begin{aligned}
\mathcal{S}(\phi) & =\bigcap\left\{K \subset M^{\mathcal{U}}: K \text { is compact and } \phi \in \mathcal{I}_{M}(K)^{\perp}\right\} \\
& =\bigcap\left\{K \subset M^{\mathcal{U}}: K \text { is compact and }\langle f, \phi\rangle=0 \text { whenever } f^{\mathcal{U}} \uparrow_{K}=0\right\}
\end{aligned}
$$

will be called the extended support of $\phi$.

Note that we are extending here the definition of $\mathcal{I}_{M}(K)$ from (3.11) to subsets of $M^{\mathcal{U}}$ in the obvious way:

$$
\mathcal{I}_{M}(K)=\left\{f \in \operatorname{Lip}_{0}(M): f^{\mathcal{U}}(\zeta)=0 \text { for all } \zeta \in K\right\} .
$$

It is straightforward to check that Definition 3.5.5 is equivalent to

$$
\mathcal{S}(\phi)=\bigcap\left\{\bar{A}^{\mathcal{U}}: A \subset M \text { and }\langle f, \phi\rangle=0 \text { whenever } f \uparrow_{A}=0\right\} .
$$

We will first prove some basic facts about the extended supports. Let us start by checking that this definition really is a generalization of supports in $\mathcal{F}(M)$ and admits a characterization similar to the one given by Proposition 3.3.10:

Proposition 3.5.6. Let $\phi \in \mathcal{F}(M)^{* *}$ and $\zeta \in M^{\mathcal{U}}$. Then $\zeta \in \mathcal{S}(\phi)$ if and only if for every neighborhood $U \subset M^{\mathcal{U}}$ of $\zeta$ there exists a function $f \in \operatorname{Lip}_{0}(M)$ whose support is contained in $U \cap M$ and such that $\langle f, \phi\rangle \neq 0$.

Proof. Suppose that $\zeta$ satisfies the condition in the statement, and let $K \subset M^{\mathcal{U}}$ be closed and such that $\phi \in \mathcal{I}_{M}(K)^{\perp}$. If $\zeta \notin K$ then let $U$ be a neighborhood of $\zeta$ such 
that $\bar{U}^{\mathcal{U}} \cap K=\varnothing$, then by assumption there is $f \in \operatorname{Lip}_{0}(M)$ supported on $U \cap M$ with $\langle f, \phi\rangle \neq 0$. But $f^{\mathcal{U}}$ vanishes on $K$ and hence $\langle f, \phi\rangle=0$, a contradiction. So $\zeta \in K$, and it follows that $\zeta \in \mathcal{S}(\phi)$.

Now suppose that $\zeta$ does not satisfy the condition. This means that there is an open neighborhood $U$ of $\zeta$ such that $\langle f, \phi\rangle=0$ for every $f \in \operatorname{Lip}_{0}(M)$ whose support is contained in $U \cap M$. Let $V$ be an open set with $\zeta \in V \subset \bar{V}^{\mathcal{U}} \subset U$ and $K=M^{\mathcal{U}} \backslash V$. Then $f \in \mathcal{I}_{M}(K)$ implies $\operatorname{supp}(f) \subset \overline{V \cap M} \subset U \cap M$ and therefore $\langle f, \phi\rangle=0$. Thus $\phi \in \mathcal{I}_{M}(K)^{\perp}$, hence $\mathcal{S}(\phi) \subset K$ and $\zeta \notin \mathcal{S}(\phi)$.

This characterization yields several basic properties of extended supports almost immediately. First of all we check that, for elements of $\mathcal{F}(M)$, supports and extended supports agree as much as possible, considering that the latter must be closed in $M^{\mathcal{U}}$.

Corollary 3.5.7. If $m \in \mathcal{F}(M)$ then $\operatorname{supp}(m)=\mathcal{S}(m) \cap M$ and $\mathcal{S}(m)=\overline{\operatorname{supp}(m)}$.

Proof. The first equality follows immediately from comparing Proposition 3.5.6 and Proposition 3.3.10. This also yields $\overline{\operatorname{supp}(m)} \mathcal{U} \subset \mathcal{S}(m)$, whereas the reverse inclusion follows from (3.20) and Proposition 3.3.9.

Recall that $\operatorname{supp}(m)$ is a separable subset of $M$ for any $m \in \mathcal{F}(M)$. We do not know whether, similarly, $\mathcal{S}(\phi)$ is a separable subset of $M^{\mathcal{U}}$ for any $\phi \in \mathcal{F}(M)^{* *}$, but we can show that its intersection with $M$ is still separable.

Proposition 3.5.8. For any $\phi \in \mathcal{F}(M)^{* *}$, the subset $\mathcal{S}(\phi) \cap M$ of $M$ is separable.

Proof. We need to show that any discrete subset of the metric space $\mathcal{S}(\phi) \cap M$ is at most countable. Let $\left\{p_{i}: i \in I\right\}$ be such a set, then there are $r_{i}>0$ such that the subsets $B\left(p_{i}, 2 r_{i}\right) \cap \mathcal{S}(\phi)$ of $M$ are pairwise disjoint. We claim that the balls $B\left(p_{i}, r_{i}\right)$ are disjoint in $M$. Indeed, suppose otherwise that there exists $x \in B\left(p_{i}, r_{i}\right) \cap B\left(p_{j}, r_{j}\right)$ for some $i \neq j \in I$. Assume $r_{i} \leq r_{j}$. Then

$$
d\left(p_{i}, p_{j}\right) \leq d\left(p_{i}, x\right)+d\left(x, p_{j}\right)<r_{i}+r_{j} \leq 2 r_{j}
$$

therefore $p_{i} \in B\left(p_{i}, 2 r_{i}\right) \cap B\left(p_{j}, 2 r_{j}\right)$, contradicting disjointness.

For each $i \in I$ consider the function $\rho_{i} \in \operatorname{Lip}(M)$ given by $\rho_{i}: x \mapsto d\left(x, p_{i}\right)$, then $U_{i}=\left(\rho_{i}^{\mathcal{U}}\right)^{-1}\left(-\infty, r_{i}\right)$ is an open neighborhood of $p_{i}$ in $M^{\mathcal{U}}$. Since $p_{i} \in$ $\mathcal{S}(\phi)$, Proposition 3.5.6 yields a function $f_{i} \in B_{\operatorname{Lip}_{0}(M)}$ such that $\left\langle f_{i}, \phi\right\rangle \neq 0$ and $\operatorname{supp}\left(f_{i}\right) \subset U_{i} \cap M \subset B\left(p_{i}, r_{i}\right)$. That is, the functions $f_{i}$ have pairwise disjoint supports. Now apply Lemma 3.2.4 to conclude that for any $k \in \mathbb{N}$ there may only exist a finite amount of indices $i \in I$ such that $\left|\left\langle f_{i}, \phi\right\rangle\right| \geq \frac{1}{k}$. Hence $I$ is countable. 
Another basic fact of extended supports is that they are compatible with finite sums:

Corollary 3.5.9. If $\phi, \psi \in \mathcal{F}(M)^{* *}$, then $\mathcal{S}(\phi+\psi) \subset \mathcal{S}(\phi) \cup \mathcal{S}(\psi)$. If moreover $\mathcal{S}(\phi) \cap \mathcal{S}(\psi)=\varnothing$, then we get an equality.

Proof. The first part of the statement is straightforward. Assume therefore that $\mathcal{S}(\phi)$ and $\mathcal{S}(\psi)$ are disjoint and let $\zeta \in \mathcal{S}(\phi)$. By Proposition 3.5.6 there exists a neighborhood $V \subset M^{\mathcal{U}}$ of $\zeta$ such that for every function $f \in \operatorname{Lip}_{0}(M)$ supported in $V \cap M$ we have $\langle f, \psi\rangle=0$. If now $U \subset M^{\mathcal{U}}$ is any neighborhood of $\zeta$, by Proposition 3.5.6 again, we may find a function $f \in \operatorname{Lip}_{0}(M)$ with the support contained in $U \cap V \cap M$ which satisfies $\langle f, \phi\rangle \neq 0$. Hence also $\langle f, \phi+\psi\rangle=\langle f, \phi\rangle \neq 0$. We conclude that $\zeta \in \mathcal{S}(\phi+\psi)$.

Compatibility of the extended support with infinite sums, or more generally with limits, requires extra hypotheses and so its discussion will be withheld until Lemma 3.5.20.

As another consequence of Proposition 3.5.6, we may extend Proposition 3.3.12 to the bidual. A similar proof works, taking the precaution not to leave the realcompactification $M^{\mathcal{R}}$ :

Corollary 3.5.10. Let $\phi, \psi$ be positive elements of $\mathcal{F}(M)^{* *}$.

(a) If $f \in \operatorname{Lip}_{0}(M), f \geq 0$ and $\langle f, \phi\rangle=0$, then $f^{\mathcal{U}}=0$ on $\mathcal{S}(\phi) \cap M^{\mathcal{R}}$.

(b) If $f \in B_{\operatorname{Lip}_{0}(M)}$ and $\langle f, \phi\rangle=\|\phi\|$, then $f^{\mathcal{U}}=\rho^{\mathcal{U}}$ on $\mathcal{S}(\phi) \cap M^{\mathcal{R}}$.

(c) If $\phi \leq \psi$ then $\mathcal{S}(\phi) \subset \mathcal{S}(\psi)$.

Proof. (a) Suppose that $f^{\mathcal{U}}(\zeta)>0$ for some $\zeta \in \mathcal{S}(\phi) \cap M^{\mathcal{R}}$. Then there are $c>0$ and an open neighborhood $U$ of $\zeta$ such that $f^{\mathcal{U}}(\xi) \geq c$ for $\xi \in U$. Moreover, since $\zeta \in M^{\mathcal{R}}$, we may choose $U$ such that $U \cap M$ is bounded. By Proposition 3.5.6 there exists $h \in \operatorname{Lip}_{0}(M)$ such that $\operatorname{supp}(h) \subset U \cap M$ and $\langle h, \phi\rangle \neq 0$. We may assume that $h \geq 0$ and $\langle h, \phi\rangle>0$ by replacing $h$ with $h^{+}$or $h^{-}$. Since $h$ is bounded, we may also assume that $h \leq c$ by multiplying by a positive constant. Then $f-h \geq 0$ but $\langle f-h, \phi\rangle<0$, a contradiction.

(b) Since $\|\phi\|=\langle\rho, \phi\rangle$, this is immediate from applying (a) to the function $\rho-f$.

(c) Fix $\zeta \in \mathcal{S}(\phi)$ and let $U$ be a neighborhood of $\zeta$. As in part (a), there is $h \in \operatorname{Lip}_{0}(M)$ such that $\operatorname{supp}(h) \subset U \cap M, h \geq 0$ and $\langle h, \phi\rangle>0$. Thus $\langle h, \psi\rangle \geq$ $\langle h, \phi\rangle>0$, and Proposition 3.5.6 implies that $\zeta \in \mathcal{S}(\psi)$.

A generalized version of inclusion (3.13) also holds for supports of weighted functionals: 
Corollary 3.5.11. Let $h \in \operatorname{Lip}(M)$ be such that $W_{h}$ is a bounded linear operator on $\operatorname{Lip}_{0}(M)$. Then for every $\phi \in \mathcal{F}(M)^{* *}$ we have $\mathcal{S}\left(\phi \circ W_{h}\right) \subset \mathcal{S}(\phi) \cap \overline{\operatorname{supp}(h)}^{\mathcal{U}}$.

Proof. Let $f \in \operatorname{Lip}_{0}(M)$ and suppose $f=0$ on $\operatorname{supp}(h)$, then $f h=0$ and so $\left\langle f, \phi \circ W_{h}\right\rangle=\langle f h, \phi\rangle=0$. Thus $\mathcal{S}\left(\phi \circ W_{h}\right) \subset \overline{\operatorname{supp}(h)} \mathcal{U}$ by (3.20). Now let $A \subset M$ be such that $\langle f, \phi\rangle=0$ for any $f \in \operatorname{Lip}_{0}(M)$ such that $f \uparrow_{A}=0$. Then, for any such $f$ we also have $\left\langle f, \phi \circ W_{h}\right\rangle=\langle f h, \phi\rangle=0$ since $(f h) \uparrow_{A}=0$, and therefore $\mathcal{S}\left(\phi \circ W_{h}\right) \subset \bar{A}^{\mathcal{U}}$ by (3.20). Taking the intersection over all such $A$ we get $\mathcal{S}\left(\phi \circ W_{h}\right) \subset \mathcal{S}(\phi)$.

\section{Localization property of extended supports}

We now turn to the localization property stated in the discussion before Definition 3.5.5. As was hinted there, one reason behind this different behaviour of supports in $\mathcal{F}(M)$ and in $\mathcal{F}(M)^{* *}$ is that a hypothetical bidual version of the intersection theorem, which would have the form

$$
\bigcap_{i} \mathcal{I}_{M}\left(K_{i}\right)^{\perp}=\mathcal{I}_{M}\left(\bigcap_{i} K_{i}\right)^{\perp}
$$

is not true in general. Indeed, if $\left\{K_{i}\right\}$ is the family of balls centered at 0 then the derivations at 0 are contained in the left hand side of (3.21) but not in the right hand side. Let us recall here that $\mathcal{I}_{M}(K)^{\perp}=\mathcal{F}_{M}(K)^{\perp \perp}$ can be identified with $\mathcal{F}(K)^{* *}$ for $K \subset M$.

We will now establish an optimal version of the intersection theorem for the bidual. In order to do that, we need to introduce a variation of the ideals $\mathcal{I}_{M}(K)$ for $K \subset M^{\mathcal{U}}$. Let us define $\mathcal{J}_{M}(K)$ as the space of all functions $f \in \mathcal{I}_{M}(K)$ that are moreover locally flat at $K$, that is, for every $\varepsilon>0$ there is an open neighborhood $U$ of $K$ such that $\left\|f \uparrow_{U \cap M}\right\|_{L} \leq \varepsilon$. This is a closed subspace of $\operatorname{Lip}_{0}(M)$, and moreover it is an ideal when $M$ is bounded. By [53, Lemma 7.43] this ideal can also be described as

$$
\mathcal{J}_{M}(K)=\overline{\bigcup\left\{\mathcal{I}_{M}(U): U \text { is an open neighborhood of } K\right\}} .
$$

With this definition in place we can now state:

Proposition 3.5.12. Suppose that $M$ is bounded, and let $\left\{K_{i}: i \in I\right\}$ be a family of closed subsets of $M^{\mathcal{U}}$. Then

$$
\mathcal{I}_{M}\left(\bigcap_{i \in I} K_{i}\right)^{\perp} \subset \bigcap_{i \in I} \mathcal{I}_{M}\left(K_{i}\right)^{\perp} \subset \mathcal{J}_{M}\left(\bigcap_{i \in I} K_{i}\right)^{\perp} .
$$


Proof. Denote $K=\bigcap_{i} K_{i}$ and $Y=\operatorname{span} \bigcup_{i} \mathcal{I}\left(K_{i}\right)$. We will prove that

$$
\mathcal{J}_{M}(K) \subset \bar{Y} \subset \mathcal{I}_{M}(K)
$$

and the result will follow by taking annihilators. The inclusion $\bar{Y} \subset \mathcal{I}_{M}(K)$ is obvious, so we only need to show that $\mathcal{J}_{M}(K) \subset \bar{Y}$.

We will first prove that fact under the additional hypothesis that $0 \in K$. Notice that each $\mathcal{I}_{M}\left(K_{i}\right)$ is an ideal, hence so are $Y$ and $\bar{Y}$. Therefore, by [53, Proposition 7.44] it is enough to check that $K$ is the "extended hull" of $\bar{Y}$, i.e. that

$$
K=\left\{\zeta \in M^{\mathcal{U}}: f^{\mathcal{U}}(\zeta)=0 \text { for all } f \in \bar{Y}\right\} .
$$

In order to prove inclusion $\subset$ in this equation, fix $\zeta \in K$. Clearly $f^{\mathcal{U}}(\zeta)=0$ for every $f \in \mathcal{I}_{M}\left(K_{i}\right)$ and hence for $f \in Y$, and the continuity of $\delta(\zeta) \in \operatorname{Lip}_{0}(M)^{*}$ shows that this is also the case for $f \in \bar{Y}$. Conversely, suppose that $\zeta \notin K$. Then there is $i \in I$ such that $\zeta \notin K_{i}$, and by Proposition 3.4.4 there are open sets $U, V \subset M^{\mathcal{U}}$ such that $\zeta \in U, K_{i} \subset V$, and $d(U \cap M, V \cap M)>0$. We may therefore find $f \in \operatorname{Lip}_{0}(M)$ such that $0 \leq f \leq 1, f=1$ on $U \cap M$, and $f=0$ on $V \cap M$ (note that $0 \in K \subset V$ ). That is, $f \in \mathcal{I}_{M}\left(K_{i}\right) \subset Y$ but $f(\zeta) \neq 0$. This completes the proof of this case.

Now suppose that $0 \notin K$, so there exists $i_{0} \in I$ such that $0 \notin K_{i_{0}}$. Proposition 3.4.4 yields open neighborhoods $U$ and $V$ of 0 and $K_{i_{0}}$, respectively, such that $d(U \cap M, V \cap M)>0$. Fix $f \in \mathcal{J}_{M}(K)$, then by McShane's theorem there is $g \in \operatorname{Lip}_{0}(M)$ such that $g=f$ on $U \cap M$ and $g=0$ on $V \cap M$. Since $K \subset K_{i_{0}} \subset V$, we have $f-g \in \mathcal{J}_{M}(K \cup\{0\})$. By the paragraph above (applied to the family $\left.\left\{K_{i} \cup\{0\}: i \in I\right\}\right)$ we deduce that $f-g \in \bar{Y}$. But clearly $g \in \mathcal{I}_{M}\left(K_{i_{0}}\right) \subset Y$, so $f \in \bar{Y}$. This ends the proof.

Note the similarity between our argument in this proof and the one employed in the first part of the proof of Theorem 3.3.5. Both are based on the algebra structure of $\operatorname{Lip}_{0}(M)$, and the main difference is that we are now dealing with norm-closed ideals instead of weak* ${ }^{*}$-closed ideals.

Proposition 3.5.12 is optimal in that either inclusion may become an equality. For instance, expanding on the derivation example given above, if $\left\{K_{i}\right\}$ consists of the closures in $M^{\mathcal{U}}$ of the balls centered on some $x \in M$ then the right inclusion is always an equality whereas the left one is if and only if $x$ is isolated. The right inclusion may also be strict e.g. when $\left\{K_{i}\right\}$ consists of a single set $\{x\}$ where $x \in M$ is not isolated. It is straightforward to combine these cases in order to obtain an example where both inclusions are strict.

We are now ready to prove the main result in this section, according to which the property that $\langle f, \phi\rangle$ depends only on the values of $f$ in a neighborhood of $\mathcal{S}(\phi)$ is valid when $\phi$ avoids infinity. We will also see in Example 3.5.15 that it does not hold in general. 
Theorem 3.5.13. Suppose that $\phi \in \mathcal{F}(M)^{* *}$ avoids infinity. If $U$ is any open subset of $M^{\mathcal{U}}$ containing $\mathcal{S}(\phi)$, then $\langle f, \phi\rangle=0$ for any $f \in \operatorname{Lip}_{0}(M)$ that vanishes on $U \cap M$. Moreover, $\mathcal{S}(\phi)$ is the smallest subset of $M^{\mathcal{U}}$ with that property.

Proof. We will prove the second statement first. Let

$$
\mathcal{Z}=\left\{K \subset M^{\mathcal{U}}: K \text { is compact and } \phi \in \mathcal{I}_{M}(K)^{\perp}\right\}
$$

so that $\mathcal{S}(\phi)=\bigcap \mathcal{Z}$. Suppose that $S^{\prime}$ is a subset of $M^{\mathcal{U}}$ satisfying the hypothesis that does not contain $\mathcal{S}(\phi)$, i.e. there exists $\zeta \in \mathcal{S}(\phi) \backslash S^{\prime}$. Let $U$ and $V$ be disjoint open sets in $M^{\mathcal{U}}$ containing $S^{\prime}$ and $\zeta$, respectively. Given $f \in \operatorname{Lip}_{0}(M)$ that vanishes on $M^{\mathcal{U}} \backslash V$, it vanishes on $U$ in particular and, by the assumption on $S^{\prime}$, we have $\langle f, \phi\rangle=0$. Thus $M^{\mathcal{U}} \backslash V \in \mathcal{Z}$ and $\zeta \in \mathcal{S}(\phi) \subset M^{\mathcal{U}} \backslash V$, a contradiction. Therefore we must have $\mathcal{S}(\phi) \subset S^{\prime}$ as we wanted to prove.

Let us now proceed with the first statement. If $M$ is bounded then this is an almost immediate consequence of Proposition 3.5.12. For the general case we will need a technical trick, similar to the one employed in the second part of the proof of Theorem 3.3.5, in order to reduce the problem to a bounded setting where Proposition 3.5.12 can be applied. Fix $n \in \mathbb{N}$ and let $A=B\left(0,2^{n+2}\right)$, and consider the two weighting operators

$$
\begin{aligned}
& W_{H_{n}}: \operatorname{Lip}_{0}(M) \rightarrow \operatorname{Lip}_{0}(M) \\
& W_{H_{n}}^{\prime}: \operatorname{Lip}_{0}(A) \rightarrow \operatorname{Lip}_{0}(M)
\end{aligned}
$$

which satisfy $W_{H_{n}}(f)=W_{H_{n}}^{\prime}\left(f \uparrow_{A}\right)$ for every $f \in \operatorname{Lip}_{0}(M)$.

Fix $K \in \mathcal{Z}$ and $g \in \mathcal{I}_{A}\left(K \cap \bar{A}^{\mathcal{U}}\right)$, and let $f=W_{H_{n}}^{\prime}(g) \cdot H_{n+1} \in \operatorname{Lip}_{0}(M)$. Clearly $W_{H_{n}}^{\prime}(g)=W_{H_{n}}^{\prime}\left(f \uparrow_{A}\right)$ as $H_{n+1}=1$ on $\operatorname{supp}\left(H_{n}\right)$, therefore

$$
\left\langle W_{H_{n}}^{\prime}(g), \phi\right\rangle=\left\langle W_{H_{n}}^{\prime}\left(f \uparrow_{A}\right), \phi\right\rangle=\left\langle W_{H_{n}}(f), \phi\right\rangle .
$$

But moreover $f \in \mathcal{I}_{M}(K)$, hence $W_{H_{n}}(f) \in \mathcal{I}_{M}(K)$ and we obtain $\left\langle g, \phi \circ W_{H_{n}}^{\prime}\right\rangle=$ 0 . We have thus proved that $\phi \circ W_{H_{n}}^{\prime} \in \mathcal{I}_{A}\left(K \cap \bar{A}^{\mathcal{U}}\right)^{\perp}$. Apply Proposition 3.5.12 in the bounded metric space $A$ to get

$$
\phi \circ W_{H_{n}}^{\prime} \in \bigcap_{K \in \mathcal{Z}} \mathcal{I}_{A}\left(K \cap \bar{A}^{\mathcal{U}}\right)^{\perp} \subset \mathcal{J}_{A}\left(\bigcap_{K \in \mathcal{Z}} K \cap \bar{A}^{\mathcal{U}}\right)^{\perp}=\mathcal{J}_{A}\left(\mathcal{S}(\phi) \cap \bar{A}^{\mathcal{U}}\right)^{\perp} .
$$

Now let $f \in \operatorname{Lip}_{0}(M)$ be such that $f=0$ on $U \cap M$ for some open neighborhood $U$ of $\mathcal{S}(\phi)$. Then $f$ is clearly locally flat on $\mathcal{S}(\phi)$, i.e. $f \in \mathcal{J}_{M}(\mathcal{S}(\phi))$ and therefore $f \uparrow_{A} \in \mathcal{J}_{A}\left(\mathcal{S}(\phi) \cap \bar{A}^{\mathcal{U}}\right)$. So we get

$$
\left\langle f, \phi \circ W_{H_{n}}\right\rangle=\left\langle f \uparrow_{A}, \phi \circ W_{H_{n}}^{\prime}\right\rangle=0 .
$$


Finally, since $\phi$ avoids infinity, taking limits as $n \rightarrow \infty$ yields $\langle f, \phi\rangle=0$.

Note that we have actually proved an apparently stronger result: if $\phi \in \mathcal{F}(M)^{* *}$ avoids infinity then $\langle f, \phi\rangle=0$ for any $f \in \operatorname{Lip}_{0}(M)$ such that $f^{\mathcal{U}}$ vanishes at $\mathcal{S}(\phi)$ and $f$ is locally flat at $\mathcal{S}(\phi)$. In fact, both statements are equivalent by [53, Lemma 7.43].

As an immediate consequence of Theorem 3.5.13 we obtain:

Corollary 3.5.14. If $\phi \in \mathcal{F}(M)^{* *}$ avoids infinity, then $\mathcal{S}(\phi)=\varnothing$ if and only if $\phi=0$.

Theorem 3.5.13 and Corollary 3.5.14 may fail in the general case as illustrated by the next example. This also shows that, unlike in $\mathcal{F}(M)$, a bounded extended support does not necessarily imply avoiding infinity, strongly or not.

Example 3.5.15. Let $M \subset \mathbb{R}^{2}$ consist of the points $x_{n}=(0, n)$ and $y_{n}=(1, n)$ for $n \geq 0$. Define $m_{n}=\delta\left(x_{n}\right) / n$ for $n \geq 1$. Then $\left\|m_{n}\right\|=1$, so we may find a subnet $\left(x_{n_{i}}\right)$ of the sequence $\left(x_{n}\right)$ that converges to some $\zeta \in M^{\mathcal{U}}$ and such that $\left(m_{n_{i}}\right)$ converges weak ${ }^{*}$ to some $\phi \in \mathcal{F}(M)^{* *}$. Notice that $\phi \geq 0$ and $\|\phi\|=\langle\rho, \phi\rangle=1$.

We claim that $\mathcal{S}(\phi)=\varnothing$. Indeed, let $X=\left\{x_{n}: n \geq 0\right\}$ and $Y=\left\{y_{n}: n \geq 0\right\}$, then $M^{\mathcal{U}}=\bar{X}^{\mathcal{U}} \cup \bar{Y}^{\mathcal{U}}$ is a disjoint union by Proposition 3.4.2 and it is clear that $\mathcal{S}(\phi) \subset$ $\bar{X}^{\mathcal{U}}$ by (3.20). Now let $U \subset M^{\mathcal{U}}$ be a neighborhood of $\bar{X}^{\mathcal{U}}$ such that $U \cap \bar{Y}^{\mathcal{U}}=\varnothing$ and suppose that $f \in \operatorname{Lip}_{0}(M)$ is supported on $U \cap M=X$. Then $f\left(y_{n}\right)=0$ and $\left|f\left(x_{n}\right)\right| \leq\|f\|_{L} d\left(x_{n}, y_{n}\right)=\|f\|_{L}$ for every $n$, hence $\langle f, \phi\rangle=\lim _{i} f\left(x_{n_{i}}\right) / n_{i}=0$. So $\mathcal{S}(\phi) \cap \bar{X}^{\mathcal{U}}=\varnothing$ by Proposition 3.5.6, therefore $\mathcal{S}(\phi)$ is empty.

Observe also that if $f \in \operatorname{Lip}_{0}(M)$ is constant on some neighborhood of $\zeta$, then in particular it is bounded on $\left(x_{n_{i}}\right)$ and $\langle f, \phi\rangle=0$ again. Hence $\phi$ is a derivation at $\zeta$.

Note that Example 3.5.15 together with Corollary 3.5.9 allow us to generate examples of functionals with non-empty extended supports failing Theorem 3.5.13. Indeed, consider for instance any functional of the form $\phi+\delta\left(x_{n}\right)$. Its extended support is $\left\{x_{n}\right\}$ and the function $\rho G_{n}$ vanishes on a neighborhood of $\left\{x_{n}\right\}$, however $\left\langle\rho G_{n}, \phi+\delta\left(x_{n}\right)\right\rangle=1$.

The functional $\phi$ constructed in Example 3.5.15 vanishes on any bounded function of $\operatorname{Lip}_{0}(M)$. This is, in fact, a general property of elements of $\mathcal{F}(M)^{* *}$ with empty extended support. Note that this also shows that the principle that "local equality implies global equality" in $\mathcal{F}(M)$ (Corollary 3.3.11) is no longer valid in $\mathcal{F}(M)^{* *}$.

Proposition 3.5.16. Let $\phi \in \mathcal{F}(M)^{* *}$ be such that $\mathcal{S}(\phi)=\varnothing$. Then $\langle f, \phi\rangle=0$ for every bounded $f \in \operatorname{Lip}_{0}(M)$. 
Proof. We will adapt an argument from [53, Lemma 7.28]. Once again let

$$
\mathcal{Z}=\left\{K \subset M^{\mathcal{U}}: K \text { is compact and } \phi \in \mathcal{I}(K)^{\perp}\right\}
$$

so that we have $\bigcap \mathcal{Z}=\mathcal{S}(\phi)=\varnothing$. For each $\zeta \in M^{\mathcal{U}}$ choose a compact $K_{\zeta} \in \mathcal{Z}$ such that $\zeta \notin K_{\zeta}$. Further, choose a compact set $K$ and an open set $V$ in $M^{\mathcal{U}}$ such that $K_{0} \subset V \subset K$ and $0 \notin K$.

For every $\zeta \in K$ we may construct a function $g_{\zeta} \in \operatorname{Lip}_{0}(M)$ such that $0 \leq g \leq 1$, $g_{\zeta}^{\mathcal{U}}(\zeta)=1$, and $g_{\zeta} \in \mathcal{I}_{M}\left(K_{\zeta}\right)$. Denote

$$
U_{\zeta}=\left\{\xi \in M^{\mathcal{U}}: g_{\zeta}^{\mathcal{U}}(\xi)>0\right\}
$$

Since $\zeta \in U_{\zeta}$, the sets $U_{\zeta}$ form an open cover of the compact $K$, and we can extract a finite subcover $U_{\zeta_{1}} \cup \ldots \cup U_{\zeta_{n}}$. Now let $g=g_{\zeta_{1}}+\ldots+g_{\zeta_{n}}$. Then we have $g^{\mathcal{U}}(\zeta)>0$ for all $\zeta \in K$, so by compactness there is a positive constant $c>0$ such that $g^{\mathcal{U}}(\zeta) \geq c$ for $\zeta \in K$. Therefore $1 / g$ is a bounded Lipschitz function on $V \cap M$. By McShane's theorem there exists a bounded $h \in \operatorname{Lip}_{0}(M)$ such that $h=1 / g$ on $V \cap M$.

To finish, let us write

$$
\langle f, \phi\rangle=\langle f-f g h, \phi\rangle+\left\langle f g_{\zeta_{1}} h, \phi\right\rangle+\ldots+\left\langle f g_{\zeta_{n}} h, \phi\right\rangle
$$

and notice that all functions involved are products of bounded Lipschitz functions, hence Lipschitz themselves. We have $f=f g h$ on $V \cap M$, thus $f-f g h \in \mathcal{I}_{M}\left(K_{0}\right)$ and we get $\langle f-f g h, \phi\rangle=0$ because $K_{0} \in \mathcal{Z}$. On the other hand, for each $i=1, \ldots, n$ we have $f g_{\zeta_{i}} h \in \mathcal{I}_{M}\left(K_{\zeta_{i}}\right)$ and so $\left\langle f g_{\zeta_{i}} h, \phi\right\rangle=0$ too. We conclude that $\langle f, \phi\rangle=0$.

The converse of Proposition 3.5.16 does not hold in general:

Example 3.5.17. Let $M \subset \mathbb{R}$ consist of 0 and the points $x_{n}=2^{n}$ for $n \in \mathbb{N}$. The functionals $\phi_{n}=\delta\left(x_{n}\right) / 2^{n}$ are uniformly bounded, so there is a subnet $\left(x_{n_{i}}\right)$ of $\left(x_{n}\right)$ such that $x_{n_{i}}$ converges to some $\zeta \in M^{\mathcal{U}}$ and $\phi_{n_{i}}$ converges weak* to some $\phi \in \mathcal{F}(M)^{* *}$. It is clear that $\langle f, \phi\rangle=0$ whenever $f \in \operatorname{Lip}_{0}(M)$ is bounded. Let us check that $\zeta \in \mathcal{S}(\phi)$. To that end, fix a neighborhood $U$ of $\zeta$. By Proposition 3.5.6 it will be enough to find $f \in \operatorname{Lip}_{0}(M)$ such that $\langle f, \phi\rangle=1$ and $\operatorname{supp}(f) \subset U \cap M$. We claim that this is satisfied by the function defined by $f(0)=0$ and

$$
f\left(x_{n}\right)= \begin{cases}2^{n} & , \text { if } x_{n} \in U \\ 0 & , \text { if } x_{n} \notin U\end{cases}
$$

for $n \in \mathbb{N}$. Indeed, it is easy to check that $\|f\|_{L} \leq 2$. Moreover $\left\langle f, \phi_{n}\right\rangle=1$ for $x_{n} \in U$, so considering a subnet of $\left(x_{n_{i}}\right)$ contained in $U$ allows us to conclude that $\langle f, \phi\rangle=1$. 
We will show now how extended supports may be used to characterize of some of the classes of functionals defined in Definition 3.5.1. The following proposition complements Proposition 3.5.2.

Proposition 3.5.18. Let $\phi \in \mathcal{F}(M)^{* *}$.

(a) $\phi$ is concentrated at infinity if and only if $\mathcal{S}(\phi) \cap M^{\mathcal{R}}=\varnothing$.

(b) $\phi$ avoids 0 strongly if and only if $0 \notin \mathcal{S}(\phi)$.

Proof. (a) Suppose that $\phi$ is concentrated at infinity. Let $\zeta \in M^{\mathcal{R}}$ and take $n \in \mathbb{N}$ such that $2^{n}>\rho^{\mathcal{U}}(\zeta)$. Then $U=\left\{\xi \in M^{\mathcal{U}}: \rho^{\mathcal{U}}(\xi)<2^{n}\right\}$ is a neighborhood of $\zeta$, and for every $f \in \operatorname{Lip}_{0}(M)$ with $\operatorname{supp}(f) \subset U$ we have $\langle f, \phi\rangle=\left\langle f H_{n}, \phi\right\rangle=$ $\left\langle f, \phi \circ W_{H_{n}}\right\rangle=0$. By Proposition 3.5.6, $\zeta \notin \mathcal{S}(\phi)$.

Now suppose that $\mathcal{S}(\phi) \cap M^{\mathcal{R}}=\varnothing$ and fix $n \in \mathbb{N}$. Then by Corollary 3.5.11

$$
\mathcal{S}\left(\phi \circ W_{H_{n}}\right) \subset \mathcal{S}(\phi) \cap \overline{\operatorname{supp}\left(H_{n}\right)} \mathcal{U} \subset \overline{B\left(0,2^{n+1}\right)}{ }^{\mathcal{U}} \backslash M^{\mathcal{R}}=\varnothing .
$$

But $\phi \circ W_{H_{n}}$ clearly avoids infinity (e.g. by Proposition 3.5.2(c)), so $\phi \circ W_{H_{n}}=0$ by Corollary 3.5.14.

(b) If $\phi$ avoids 0 strongly then 0 cannot be contained in $\mathcal{S}(\phi)=\mathcal{S}\left(\phi \circ W_{G_{-n}}\right) \subset$ $\overline{\operatorname{supp}\left(G_{-n}\right)} \mathcal{U}$ by Corollary 3.5.11. For the converse, assume $0 \notin \mathcal{S}(\phi)$. By Proposition 3.5.6 there is a neighborhood $U \subset M^{\mathcal{U}}$ of 0 such that $\langle f, \phi\rangle=0$ for every $f \in \operatorname{Lip}_{0}(M)$ supported in $U \cap M$. Let $n \in \mathbb{N}$ be so large that $\operatorname{supp}\left(H_{-n}\right) \subset U \cap M$. Then for every $f \in \operatorname{Lip}_{0}(M)$ we have

$$
\left\langle f, \phi \circ W_{G_{-n}}\right\rangle=\langle f, \phi\rangle-\left\langle f, \phi \circ W_{H_{-n}}\right\rangle=\langle f, \phi\rangle-\left\langle f H_{-n}, \phi\right\rangle=\langle f, \phi\rangle
$$

because $\operatorname{supp}\left(f H_{-n}\right) \subset U \cap M$. Hence $\phi=\phi \circ W_{G_{-n}}$.

It is not possible to characterize all the classes in Definition 3.5.1 via the extended supports since a functional may have a component concentrated at infinity which cannot be detected in its support (add for instance the functional from Example 3.5.15 with empty extended support to any element of $\mathcal{F}(M)^{* *}$ and apply Corollary 3.5.9). However, under the assumption of avoiding infinity there is a mutual correspondence between the extended support of a functional and its classification. The next two propositions describe that link.

Proposition 3.5.19. If $\phi \in \mathcal{F}(M)^{* *}$ avoids infinity strongly, then $\mathcal{S}(\phi) \subset M^{\mathcal{R}}$. If $\phi$ avoids infinity then the converse is also true.

Proof. Suppose that $\phi \in \mathcal{F}(M)^{* *}$ avoids infinity strongly, i.e. $\phi=\phi \circ W_{H_{n}}$ for some $n \in \mathbb{N}$. In view of Corollary 3.5.11 then

$$
\mathcal{S}(\phi)=\mathcal{S}\left(\phi \circ W_{H_{n}}\right) \subset \overline{\operatorname{supp}\left(H_{n}\right)}{ }^{\mathcal{U}} \subset M^{\mathcal{R}} .
$$


For the converse, assume that $\phi$ avoids infinity and that $\mathcal{S}(\phi) \subset M^{\mathcal{R}}$. Since $\mathcal{S}(\phi)$ is compact and $\rho^{\mathcal{U}} \Gamma_{M^{\mathcal{R}}}$ is a real-valued continuous function, it is bounded on $\mathcal{S}(\phi)$ and there exists $n \in \mathbb{N}$ such that $U=\left\{\zeta \in M^{\mathcal{U}}: \rho^{\mathcal{U}}(\zeta)<2^{n}\right\}$ is an open neighborhood of $\mathcal{S}(\phi)$. For every $f \in \operatorname{Lip}_{0}(M)$ we thus get by Theorem 3.5.13 that

$$
\langle f, \phi\rangle=\left\langle f H_{n}, \phi\right\rangle=\left\langle f, \phi \circ W_{H_{n}}\right\rangle
$$

because $f=f H_{n}$ on $U$. Hence $\phi=\phi \circ W_{H_{n}}$.

For the second proposition, we need an independent lemma describing the stability of extended supports with respect to weak* limits:

Lemma 3.5.20. Let $\left(\phi_{n}\right)$ be a sequence of functionals in $\mathcal{F}(M)^{* *}$ that avoid infinity, and suppose that $\phi_{n} \stackrel{w^{*}}{\longrightarrow} \phi \in \mathcal{F}(M)^{* *}$. Then $\mathcal{S}(\phi) \subset \overline{\bigcup_{n} \mathcal{S}\left(\phi_{n}\right)}$.

Proof. Let $\zeta \notin{\overline{U_{n} \mathcal{S}\left(\phi_{n}\right)}}^{\mathcal{U}}$ and let $U$ and $V$ be disjoint open neighborhoods of $\zeta$ and $\overline{\bigcup_{n} \mathcal{S}\left(\phi_{n}\right)} \mathcal{U}$, respectively. Then for any $f \in \operatorname{Lip}_{0}(M)$ with $\operatorname{supp}(f) \subset U$ we have by Theorem 3.5.13 that $\left\langle f, \phi_{n}\right\rangle=0$ for every $n \in \mathbb{N}$. Hence also $\langle f, \phi\rangle=$ $\lim _{n \rightarrow \infty}\left\langle f, \phi_{n}\right\rangle=0$ which proves that $\zeta \notin \mathcal{S}(\phi)$ by Proposition 3.5.6.

Proposition 3.5.21. If $\phi \in \mathcal{F}(M)^{* *}$ avoids infinity then $\mathcal{S}(\phi)=\overline{\mathcal{S}(\phi) \cap M^{\mathcal{R}}}$.

Proof. One inclusion is obvious. For the other one, recall that

$$
\mathcal{S}\left(\phi \circ W_{H_{n}}\right) \subset \mathcal{S}(\phi) \cap \overline{B\left(0,2^{n+1}\right)} \mathcal{U} \subset \mathcal{S}(\phi) \cap M^{\mathcal{R}}
$$

according to Corollary 3.5.11. Applying Lemma 3.5.20 to $\phi=\lim _{n \rightarrow \infty} \phi \circ W_{H_{n}}$ we conclude that $\mathcal{S}(\phi) \subset \overline{\mathcal{S}(\phi) \cap M^{\mathcal{R}}}$ as desired.

Compare Proposition 3.5.21 with Corollary 3.5.7: if $\phi \in \mathcal{F}(M)^{* *}$ is weak ${ }^{*}$ continuous then its extended support is completely determined by its intersection with $M$, whereas if $\phi$ avoids infinity then it is completely determined by its intersection with $M^{\mathcal{R}}$.

Let us end this chapter by identifying those functionals in $\mathcal{F}(M)^{* *}$ which are supported on just one point. In $\mathcal{F}(M)$, these are easily seen to be just the (multiples of) evaluations on said point. In the case of $\mathcal{F}(M)^{* *}$ the situation is more complicated, since we have evaluations (on elements of $M^{\mathcal{R}}$ this time) but also derivations. The next proposition states that, when we restrict ourselves to the functionals that avoid infinity, these cover all possible cases.

Proposition 3.5.22. Suppose that $\phi \in \mathcal{F}(M)^{* *}$ avoids infinity and let $\zeta \in M^{\mathcal{R}}$. Then the following are equivalent: 
(i) $\mathcal{S}(\phi) \subset\{\zeta\}$,

(ii) $\phi=a \delta(\zeta)+\psi$, where $a \in \mathbb{R}$ and $\psi$ is a derivation at $\zeta$.

Proof. First assume (ii), and let $\xi \in M^{\mathcal{U}}, \xi \neq \zeta$. Choose neighborhoods $U$, $V$ of $\xi, \zeta$ such that $d(U \cap M, V \cap M)>0$. Suppose $f \in \operatorname{Lip}_{0}(M)$ is such that $\operatorname{supp}(f) \subset U \cap M$. Then $f^{\mathcal{U}}=0$ on $V$, so $\langle f, \psi\rangle=0$ by definition and so $\langle f, \phi\rangle=0$. By Proposition 3.5.6 we get $\xi \notin \mathcal{S}(\phi)$, and (i) follows.

Now assume (i). First suppose $\zeta=0$, and let $f \in \operatorname{Lip}_{0}(M)$ be such that $f^{\mathcal{U}}$ is constant in a neighborhood $U$ of 0 . This constant must obviously be 0 . By Theorem 3.5.13, we have $\langle f, \phi\rangle=0$. This means that $\phi$ is a derivation at 0 .

Suppose now that $\zeta \neq 0$, and choose $h \in \operatorname{Lip}_{0}(M)$ such that $h^{\mathcal{U}}=1$ in a neighborhood of $\zeta$; e.g. take $h=\left(\frac{1}{b} \rho\right) \wedge 1$ where $b<\rho^{\mathcal{U}}(\zeta)$. Let $\psi=\phi-a \delta(\zeta)$ where $a=\langle h, \phi\rangle$. Then $\psi$ is a derivation at $\zeta$. Indeed, suppose $f \in \operatorname{Lip}_{0}(M)$ is such that $f^{\mathcal{U}}$ takes a constant value $c$ in a neighborhood $U$ of $\zeta$. Let $g=f-c h$, then $g^{\mathcal{U}}=0$ on $U$, and $\langle g, \phi\rangle=0$ by Theorem 3.5.13, so

$$
\langle f, \psi\rangle=\langle f, \phi\rangle-a f^{\mathcal{U}}(\zeta)=\langle f, \phi\rangle-a c=\langle f-c h, \phi\rangle=\langle g, \phi\rangle=0 .
$$

Note that we do not need the hypothesis that $\phi$ avoids infinity for the implication (ii) $\Rightarrow$ (i). In fact, one easily checks that any $\phi$ satisfying (ii) for some $\zeta \in M^{\mathcal{R}}$ avoids infinity strongly.

Corollary 3.5.23. Suppose that $\phi \in \mathcal{F}(M)^{* *}$ avoids infinity. Then $\mathcal{S}(\phi)$ is finite if and only if $\phi$ is a finite linear combination of point evaluations and derivations on points of $M^{\mathcal{U}}$.

Proof. If $\mathcal{S}(\phi)$ is finite, then by virtue of Proposition 3.5.21 we have $\mathcal{S}(\phi) \subset M^{\mathcal{R}}$. Denote $\mathcal{S}(\phi)=\left\{\zeta_{1}, \ldots, \zeta_{k}\right\}$ and find neighborhoods $U_{1}, \ldots, U_{k}$ of $\zeta_{1}, \ldots, \zeta_{k}$ with pairwise disjoint closures. For each $i$ define $h_{i} \in \operatorname{Lip}_{0}(M)$ such that $h_{i}^{\mathcal{U}}=1$ on $U_{i}$ and $h_{i}^{\mathcal{U}}=0$ on $\bigcup_{j \neq i} U_{j}$. Since $\max _{i} \rho^{\mathcal{U}}\left(\zeta_{i}\right)<\infty$, we may moreover take $h_{i}$ to have a bounded support. By Theorem 3.5.13 then

$$
\phi=\phi \circ W_{\sum_{i=1}^{k} h_{i}}=\sum_{i=1}^{k} \phi \circ W_{h_{i}} .
$$

But Corollary 3.5.11 gives that $\mathcal{S}\left(\phi \circ W_{h_{i}}\right) \subset\left\{\zeta_{i}\right\}$, so Proposition 3.5.22 yields

$$
\phi \circ W_{h_{i}}=a_{i} \delta\left(\zeta_{i}\right)+\psi_{i}
$$

for some $a \in \mathbb{R}$ and $\psi_{i}$ a derivation at $\zeta_{i}$, and the forward implication follows. On the other hand, if $\phi$ avoiding infinity is a nontrivial finite linear combination of 
evaluations and derivations, then these also avoid infinity by the uniqueness of the decomposition in Theorem 3.5.3 and Proposition 3.5.22 implies that their supports are singletons. An appeal to Corollary 3.5.9 yields that $\mathcal{S}(\phi)$ is finite.

Combining Proposition 3.5.22 with Corollary 3.5.7 immediately implies the following, that can also be proved by a direct argument:

Corollary 3.5.24. If $\phi \in \mathcal{F}(M)$ is a derivation then $\phi=0$.

Note that this already provides us with an order-theoretic property that is not invariant under changes of base point: $\mathcal{F}(M)^{* *} \backslash \mathcal{F}(M)$ contains positive functionals supported on just one point if and only if the base point of $M$ is not isolated. We will see a stronger version of this behavior in Corollary 4.3.10.

\subsection{Open problems}

We have seen in Proposition 3.1.3 how Kalton's original decomposition from [40], or rather our proposed modification thereof, allows us to express every functional in $\mathcal{F}(M)$ as an absolutely convergent series of elements with bounded supports. More generally, by Theorem 3.5.3 this is also valid for every element of $\mathcal{F}(M)^{* *}$ that avoids infinity. However, the supports of the series terms have a very specific form: they are annuli centered at the base point, with growing sizes. So we may ask:

Problem 3.1. Are there more general forms of the series decomposition (3.6) where the supports of individual terms are allowed to have other shapes?

For instance, one could want the terms to be supported on balls of a given size, or on arbitrary balls. The existence of such a decomposition would likely have consequences in the study of approximation properties, and more generally in the study of the structure of the bidual $\mathcal{F}(M)^{* *}$. Here we remark that, in any case, these decompositions can only be valid for functionals that avoid infinity by Proposition 3.5.2(a). More general decompositions could also be used to reduce certain properties of $\mathcal{F}(M)$ to the corresponding properties on $\mathcal{F}(A)$ for certain subsets $A$ of $M$, in line with the results in $[2,27]$.

A second open question concerns the following weakening of the concept of normal functional: let us say that $\phi \in \mathcal{F}(M)^{* *}$ is sequentially normal if $\left\langle f_{n}, \phi\right\rangle \rightarrow 0$ for any sequence $\left(f_{n}\right)$ of non-negative functions in $B_{\operatorname{Lip}_{0}(M)}$ that decreases pointwise to 0 . This is the same condition as for normal elements, except that nets are replaced with sequences.

Problem 3.2. Is sequential normality equivalent to normality (and hence to weak* continuity, by Theorem 3.2.5)? 
For separable $M$ the answer is positive since $B_{\operatorname{Lip}_{0}(M)}$ is then weak* metrizable. We do not know what happens in the nonseparable case, but we suspect that the answer is negative. A counterexample for Problem 3.2 is likely to answer (negatively) the following question, too:

Problem 3.3. Is $\mathcal{S}(\phi)$ separable for every $\phi \in \mathcal{F}(M)^{* *}$ ?

Proposition 3.5.8, showing that $\mathcal{S}(\phi) \cap M$ is separable, provides a partial positive answer to that question.

Our next problem is related to the intersection theorem. Although the result concerns exclusively the linear structure of Lipschitz and Lipschitz-free spaces, in our proof we have required some non-trivial facts about the algebra structure of Lipschitz spaces, namely Theorem 3.3.2. Since the result seems of a fundamental nature, it is natural to ask whether there is a more direct proof that avoids such algebraic detours, e.g. by using a method based on Lipschitz partitions of unity instead.

Problem 3.4. Find a direct proof of the intersection theorem (Theorem 3.3.5) that does not depend on the algebra structure of $\operatorname{Lip}_{0}(M)$.

The following question concerns our proposed definition of extended support for elements $\phi \in \mathcal{F}(M)^{* *}$. We have already argued that $\mathcal{S}(\phi)$ must be defined as a subset of $M^{\mathcal{U}}$, and that it cannot define a "sharp region" to which the restriction of $f \in \operatorname{Lip}_{0}(M)$ completely determines the value of $\langle f, \phi\rangle$ in the sense of Proposition 3.3.9(iii), having to settle on a "soft region" in the sense of Theorem 3.5.13 instead. With these restrictions in mind, our proposed notion successfully extends supports for $\mathcal{F}(M)$ and has all expected properties for functionals that avoid infinity (more interesting consequences will be obtained in Chapter 4). But it seems to break down when we consider functionals that do not avoid infinity or even are concentrated at infinity. We do not know whether this issue can be solved with an alternative definition, or if one can prove that every reasonable definition of extended support must fail at infinity.

Problem 3.5. Does there exist a notion of extended support in $\mathcal{F}(M)^{* *}$ that satisfies all expected properties even for functionals that are concentrated at infinity?

To finish this list of questions, let us mention a classical, probably difficult open problem concerning the bidual $\mathcal{F}(M)^{* *}$. It is our hope that the machinery of extended supports can be used to obtain advances towards its solution:

Problem 3.6. For which metric spaces $M$ is $\mathcal{F}(M)$ complemented in its bidual?

Let us note that it would be enough to show that $\mathcal{F}(M)$ is complemented in the space $Y$ of all functionals of $\mathcal{F}(M)^{* *}$ that avoid infinity, since $Y$ is a 1complemented subspace of $\mathcal{F}(M)^{* *}$ by Theorem 3.5.3. 
Not all metric spaces $M$ satisfy the property from Problem 3.6. Indeed, if $M$ is separable and $\mathcal{F}(M)$ contains $c_{0}$ (e.g. for $M=c_{0}$ ) then an easy application of Sobczyk's and Phillips' theorems shows that $\mathcal{F}(M)$ cannot be complemented in $\mathcal{F}(M)^{* *}$. On the other hand, the answer is positive when $M$ is a finite-dimensional Banach space or a subset thereof with nonempty interior [16]. For other cases, the answer is not known. The case $M=\ell_{1}$ is particularly important, as a positive answer would imply that any Banach space that is bi-Lipschitz equivalent to $\ell_{1}$ is actually isomorphic to $\ell_{1}$; see [31, Problem 16]. 


\section{Chapter 4}

\section{Integral representation}

The properties of supports in $\mathcal{F}(M)$ reviewed in Chapter 3, together with the fact that measures also act (by integration) as functionals on Lipschitz spaces, make it natural to analyze the relationship with spaces of measures defined on the metric space $M$. Some previously known results include the following: every finite Borel measure on $M$ with compact support represents an element of $\mathcal{F}(M)$ [30, p. 123], but not all elements of $\mathcal{F}(M)$ can be represented in that way when $M$ is an infinite compact [53, Theorem 3.19]. Moreover, every positive element in $\mathcal{F}(M)$ is shown in [9, Proposition 2.7] to be representable as a positive Borel measure on $M$ (a typo incorrectly specifies a probability measure; in fact, the measure does not even have to be finite as we will show). A similar result holds, with finite measures, for other function spaces related to Lipschitz spaces [34].

This chapter is devoted to exploring these topics, and we provide general forms of all statements given above. We also provide justification for our choice of the name "support", as we shall see that this notion coincides with the usual one for elements of $\mathcal{F}(M)$ that can be identified with measures. Moreover, we extend this analysis to functionals in $\mathcal{F}(M)^{* *}$ and measures on $M^{\mathcal{U}}$. Studying this matter leads to the recognition of the uniform compactification as the appropriate setting for the analysis of $\mathcal{F}(M)^{* *}$ among other enlightening ideas.

This study also leads to a number of results on majorizable elements of $\mathcal{F}(M)$ and $\mathcal{F}(M)^{* *}$, as we obtain the unexpected result that they almost coincide with those functionals that can be represented as a measure. This has some important consequences such as the weak* continuity of most majorizable functionals when $M$ is compact, or the existence of canonical "Jordan decompositions" of majorizable functionals as difference of positive functionals. We end the chapter by characterizing the metric spaces $M$ such that every element of $\mathcal{F}(M)$ is majorizable. 
All results presented in this chapter may be found in the preprint [3].

\subsection{Radon measures}

We start by introducing the basic measure-theoretic concepts used in this chapter. It is important to mention that the terminology is not universal, and there are conflicting notions of regularity and Radonness in the main references, e.g. [12, 47]. We have checked carefully that all referenced results really hold under our terminology of choice.

Let $X$ be a Hausdorff topological space and let $\mu$ be a Borel measure on $X$, i.e. a measure defined on the Borel $\sigma$-algebra of $X$. If $\mu$ is positive (but not necessarily finite) then we will say that it is

- inner regular if $\mu(E)=\sup \{\mu(K): K \subset E$ compact $\}$ for every Borel set $E \subset X$,

- outer regular if $\mu(E)=\inf \{\mu(U): U \supset E$ open $\}$ for every Borel set $E \subset X$,

- regular if it is both inner and outer regular,

- Radon if it is regular and finite.

Notice that if $\mu$ is finite then inner regularity implies regularity, and if additionally $X$ is compact then each of the four conditions above implies the other three.

If $\mu$ is a finite signed (real-valued) measure instead, we define its positive and negative variation by

$$
\begin{aligned}
& \mu^{+}(E)=\sup \{\mu(A): A \subset E \text { Borel }\} \\
& \mu^{-}(E)=\sup \{-\mu(A): A \subset E \text { Borel }\}
\end{aligned}
$$

for any Borel set $E \subset X$, and its total variation as $|\mu|=\mu^{+}+\mu^{-}$. Then $\mu^{+}, \mu^{-}$ and $|\mu|$ are finite positive Borel measures on $X$, and $\mu$ is said to have each of the four regularity properties above when the total variation measure $|\mu|$ does. We also have

$$
\mu=\mu^{+}-\mu^{-}
$$

This expression is called the Jordan decomposition of $\mu$, and satisfies the following property: if we have $\mu=\lambda^{+}-\lambda^{-}$for a pair $\lambda^{+}, \lambda^{-}$of finite positive Borel measures on $X$, then $\lambda^{+} \geq \mu^{+}$and $\lambda^{-} \geq \mu^{-}$. Moreover $\mu^{+}, \mu^{-}$are unique with respect to that property.

The support of a measure $\mu$ is defined as the set $\operatorname{supp}(\mu)$ of points $x \in X$ such that $|\mu|(U)>0$ for every open neighborhood $U$ of $x$. It is always a closed set. If $\mu$ is Radon, then $\operatorname{supp}(\mu)=\varnothing$ if and only if $\mu=0$ (see e.g. [12, Theorem 7.2.9]). Let us also mention that, when $X$ is metrizable, the support of every finite Borel measure on $X$ is separable (see e.g. [42, Lemma 2.1]). 
Given a Borel measure $\mu$ on $X$ and a Borel set $A \subset X$, we will denote by $\mu \uparrow_{A}$ the restriction of $\mu$ to $A$ defined by

$$
\mu \uparrow_{A}(E)=\mu(E \cap A)
$$

for any Borel set $E \subset X$. This is again a Borel measure on $X$. We will say that $\mu$ is concentrated on $A$ if $\mu=\mu \uparrow_{A}$, or, equivalently, $|\mu|(X \backslash A)=0$. Let us recall that, for any $\mu$, there is at least one Hahn decomposition of $X$ associated to $\mu$, i.e. a partition $X=A^{+} \cup A^{-}$into two disjoint Borel subsets such that $\mu^{+}$and $\mu^{-}$are concentrated on $A^{+}$and $A^{-}$, respectively.

In our analysis, we will consider both positive and signed Borel measures. Signed measures will have finite total variation, but positive measures may be finite or $\sigma$ finite. Measures will not be assumed to be finite, positive, or regular unless stated explicitly. We will moreover restrict ourselves to the cases where $X$ is either a metric space or a compact space, namely $M$ and $M^{\mathcal{U}}$. When the underlying space is $M$, then any finite Borel measure $\mu$ is automatically outer regular, and if $M$ is complete and separable then $\mu$ is also Radon (see Theorems 1.4.8 and 7.1.7 in [12], where a slightly different notation is used).

We will pay special attention to Radon measures, and denote by $\mathcal{M}(M)$ and $\mathcal{M}\left(M^{\mathcal{U}}\right)$ the Banach spaces of Radon measures on $M$ and $M^{\mathcal{U}}$, respectively. Recall that, since $M^{\mathcal{U}}$ is compact, $\mathcal{M}\left(M^{\mathcal{U}}\right)$ is the dual of the space $C\left(M^{\mathcal{U}}\right)$ of continuous functions on $M^{\mathcal{U}}$, which by Proposition 3.4.3 can be identified with the space of bounded, uniformly continuous functions on $M$. Let us remark once again that finite Borel measures on $M$ or $M^{\mathcal{U}}$ are Radon as soon as they are inner regular.

It is possible to identify $\mathcal{M}(M)$ with the subspace of $\mathcal{M}\left(M^{\mathcal{U}}\right)$ consisting of those measures that are concentrated on $M$. This follows easily from the fact that $M$ is always a Borel subset of $M^{\mathcal{U}}$ (it is in fact a $G_{\delta}$ subset, as we assume $M$ to be complete) and that a subset $K \subset M$ is compact as a topological subspace of $M$ if and only if it is compact as a topological subspace of $M^{\mathcal{U}}$. We will make use of this identification without further notice.

We will, in fact, consider a slightly more general class than the Radon measures:

Definition 4.1.1. Let us say that a Borel measure $\mu$ on $M$ (resp. $M^{\mathcal{U}}$ ) is almost Radon if $\mu(\{0\})=0$ and, for every closed subset $K$ of $M$ (resp. $M^{\mathcal{U}}$ ) such that $0 \notin K$, the restriction $\mu \uparrow_{K}$ is Radon.

It is straightforward to check that every almost Radon measure $\mu$ on $M$ is inner regular by noticing that

$$
\mu(E)=\lim _{n \rightarrow \infty} \mu\left(E \backslash B\left(0,2^{-n}\right)\right)=\lim _{n \rightarrow \infty} \mu \uparrow_{M \backslash B\left(0,2^{-n}\right)}(E)
$$

for every Borel set $E \subset M$, and similarly for $M^{\mathcal{U}}$ (note that we use the condition $\mu(\{0\})=0$ here). Thus, almost Radon measures are either signed Radon measures 
or $\sigma$-finite positive measures that are Radon except for a "singularity" at the base point. We must exclude the case of non-finite signed measures because of indeterminacies of the form $\infty-\infty$ around the base point - instead, such objects will be treated as the difference between two almost Radon measures.

Notice that, for an almost Radon measure $\mu$, we have $\operatorname{supp}(\mu)=\varnothing$ if and only if $\mu=0$. Indeed, for every closed set $K$ not containing 0 we have $\operatorname{supp}\left(\mu \Upsilon_{K}\right) \subset$ $\operatorname{supp}(\mu)=\varnothing$ and hence $\mu \uparrow_{K}=0$ as $\mu \uparrow_{K}$ is Radon. Also, any almost Radon measure on $M$ has separable support.

It is also easy to see that every almost Radon measure can be expressed canonically as a limit of Radon measures. Let us state and prove it explicitly for later reference:

Lemma 4.1.2. Let $\mu$ be an almost Radon measure on $M^{\mathcal{U}}$, and for $n \in \mathbb{N}$ let $\mu_{n}$ be defined by $d \mu_{n}=G_{-n}^{\mathcal{U}} d \mu$. Then

(a) each $\mu_{n}$ is Radon,

(b) $\mu_{n}$ converges setwise to $\mu$, and

(c) if $\mathcal{L} \mu \in \mathcal{F}(M)^{* *}$ then $\mathcal{L} \mu_{n}=\mathcal{L} \mu \circ W_{G_{-n}}$.

Proof. Notice that $d \mu_{n}=G_{-n}^{\mathcal{U}} d\left(\mu \uparrow_{R_{n}}\right)$ where $R_{n}=\left\{\zeta \in M^{\mathcal{U}}: \rho^{\mathcal{U}}(\zeta) \geq 2^{-n}\right\}$, therefore $\mu \uparrow_{R_{n}}$ and $\mu_{n}$ are Radon. This establishes (a), and (c) is obvious. For statement (b), observe that $\left(G_{-n}^{\mathcal{U}}\right)$ converges pointwise and increasingly to the characteristic function of the set $M^{\mathcal{U}} \backslash\{0\}$. Therefore, for any Borel set $E \subset M^{\mathcal{U}}$ we have

$$
\mu(E)=\mu(E \backslash\{0\})=\int_{M^{\mathcal{U}} \backslash\{0\}} \chi_{E} d \mu=\lim _{n \rightarrow \infty} \int_{M^{\mathcal{U}} \backslash\{0\}} \chi_{E} \cdot G_{-n}^{\mathcal{U}} d \mu=\lim _{n \rightarrow \infty} \mu_{n}(E)
$$

where $\chi_{E}$ is the characteristic function of $E$. Indeed, this follows from either of Lebesgue's convergence theorems (monotone or dominated) depending on whether $\mu$ is positive or Radon.

\subsection{Functionals induced by measures}

In this section we will carry out an analysis of the relationship between measures and functionals on Lipschitz spaces. We will determine which functionals admit an integral representation with a measure, which measures give rise to such functionals, and the relationship between the properties of measure and functional in that case.

Let us start with the observation that a Borel measure $\mu$ defined on a complete metric space $M$ acts by integration on measurable functions, in particular on 
Lipschitz functions, and thus we may define a formal mapping $\mathcal{L} \mu$ from $\operatorname{Lip}_{0}(M)$ to the reals or extended reals by

$$
\mathcal{L} \mu(f)=\int_{M} f d \mu
$$

for $f \in \operatorname{Lip}_{0}(M)$; at least, for those $f$ that are $\mu$-integrable. More generally, if $\mu$ is a Borel measure on the uniform compactification $M^{\mathcal{U}}$ then we denote

$$
\mathcal{L} \mu(f)=\int_{M^{\mathcal{U}}} f^{\mathcal{U}} d \mu
$$

for $f \in \operatorname{Lip}_{0}(M)$. Note that if the integral $\int_{M} f d \mu$ (resp. $\int_{M^{\mathcal{U}}} f^{\mathcal{U}} d \mu$ ) exists for every $f$ then this defines a linear functional $\mathcal{L} \mu$ on $\operatorname{Lip}_{0}(M)$. Let us remark here that the identification of measures on $M$ as measures on $M^{\mathcal{U}}$ is consistent with this notation.

We will say that a functional $\phi$ in $\mathcal{F}(M)$ or $\mathcal{F}(M)^{* *}$ is induced or represented by a measure $\mu$ if $\phi=\mathcal{L} \mu$. We shall use the notation $\delta_{\zeta}$ for the Dirac measure on $\zeta \in M^{\mathcal{U}}$; note the difference with the functional $\delta(\zeta)$ of $\mathcal{F}(M)^{* *}$ (if $\zeta \in M^{\mathcal{R}}$ ), which is obviously induced by $\delta_{\zeta}$. Let us remark that the measure $\delta_{0}$ induces the null functional because all $f \in \operatorname{Lip}_{0}(M)$ vanish at the base point. Thus, any functional that is represented by a measure $\mu$ is also represented by a measure

$$
\hat{\mu}=\mu-\mu(\{0\}) \cdot \delta_{0}=\mu \Upsilon_{M^{\mathcal{U}} \backslash\{0\}}
$$

such that $\hat{\mu}(\{0\})=0$. We shall denote by $\mathcal{M}_{0}(M)$ and $\mathcal{M}_{0}\left(M^{\mathcal{U}}\right)$ the spaces of such Radon measures with no mass at the base point.

\section{Measures that induce continuous functionals}

Let us start by identifying those measures that induce bounded functionals on $\operatorname{Lip}_{0}(M)$. The next two propositions show that they are precisely the measures with "finite first moment". This generalizes the first part of [9, Proposition 2.7]; see also [34, Lemma 3.3].

Proposition 4.2.1. Let $\mu$ be a Borel measure on $M^{\mathcal{U}}$ such that $\mu(\{0\})=0$. Then the following are equivalent:

(i) $\mathcal{L} \mu \in \mathcal{F}(M)^{* *}$,

(ii) $\int_{M^{\mathcal{U}}}\left|f^{\mathcal{U}}\right| d|\mu|<\infty$ for every $f \in \operatorname{Lip}_{0}(M)$,

(iii) $\int_{M^{\mathcal{U}}} \rho^{\mathcal{U}} d|\mu|<\infty$.

If they hold then $\mu$ is concentrated on $M^{\mathcal{R}},|\mu|(K)<\infty$ for any closed $K \subset M^{\mathcal{U}}$ such that $0 \notin K$, and $\mu$ is $\sigma$-finite. If $\mu$ is moreover inner regular then it is almost Radon. 
Note that the equivalence (i) $\Leftrightarrow$ (ii) may be regarded as a principle of uniform boundedness: if $\mathcal{L} \mu$ is pointwise finite, i.e. if it correctly defines a linear functional, then it is automatically continuous.

Proof. The implication (i) $\Rightarrow$ (ii) is obvious, as $\langle f, \mathcal{L} \mu\rangle=\int_{M^{\mathcal{U}}} f^{\mathcal{U}} d \mu$ is finite for every $f \in \operatorname{Lip}_{0}(M)$. (ii) $\Rightarrow$ (iii) is also obvious taking $f=\rho$. Now assume (iii), then for every $f \in \operatorname{Lip}_{0}(M)$ we have

$$
|\langle f, \mathcal{L} \mu\rangle|=\left|\int_{M^{\mathcal{U}}} f^{\mathcal{U}} d \mu\right| \leq \int_{M^{\mathcal{U}}}\left|f^{\mathcal{U}}\right| d|\mu| \leq\|f\|_{L} \cdot \int_{M^{\mathcal{U}}} \rho^{\mathcal{U}} d|\mu|
$$

hence we get (i) with $\|\mathcal{L} \mu\| \leq \int_{\mathcal{M}^{\mathcal{U}}} \rho^{\mathcal{U}} d|\mu|$. In particular, $\rho^{\mathcal{U}}<\infty$ a.e.( $\left.|\mu|\right)$ and therefore $\mu$ is concentrated on $M^{\mathcal{R}}$. Moreover, if $K$ is a closed subset of $M^{\mathcal{U}}$ such that $0 \notin K$, then there is $r>0$ such that $\rho^{\mathcal{U}} \geq r$ on $K$ and so

$$
\int_{M^{\mathcal{U}}} \rho^{\mathcal{U}} d|\mu| \geq \int_{K} \rho^{\mathcal{U}} d|\mu| \geq r \cdot|\mu|(K)
$$

hence $|\mu|(K)<\infty$. Since $M^{\mathcal{U}} \backslash\{0\}=\bigcup_{n \in \mathbb{N}} K_{n}$ where $K_{n}=\left\{\zeta \in M^{\mathcal{U}}: \rho^{\mathcal{U}}(\zeta) \geq \frac{1}{n}\right\}$ and $\mu(\{0\})=0$, we get that $\mu$ is $\sigma$-finite. The last statement is obvious.

In the version for measures on $M$, we get additional information:

Proposition 4.2.2. Let $\mu$ be a Borel measure on $M$ such that $\mu(\{0\})=0$. Suppose that either $M$ is separable or $\mu$ is inner regular. Then the following are equivalent:

(i) $\mathcal{L} \mu \in \mathcal{F}(M)^{* *}$,

(ii) $\mathcal{L} \mu \in \mathcal{F}(M)$,

(iii) $\int_{M}|f| d|\mu|<\infty$ for every $f \in \operatorname{Lip}_{0}(M)$,

(iv) $\int_{M} \rho d|\mu|<\infty$,

(v) $\mathcal{L} \mu=\int_{M} \delta(x) d \mu(x)$ as a Bochner integral.

If they hold then $\mu$ is almost Radon.

Proof. By considering $\mu$ as a Borel measure on $M^{\mathcal{U}}$ that is concentrated on $M$, the equivalence of (i), (iii) and (iv) follows from Proposition 4.2.1, as does the last statement if $\mu$ is inner regular. If $M$ is assumed to be separable instead then every closed $K \subset M$ with $0 \notin K$ is separable and complete (recall that we assume $M$ to be complete) and hence the finiteness of $\mu \uparrow_{K}$ implies that it is Radon, thus $\mu$ is again almost Radon. 
The implication (ii) $\Rightarrow(\mathrm{i})$ is obvious, as is $(\mathrm{v}) \Rightarrow(\mathrm{ii})$ since the integrand $\delta(x)$ belongs to $\mathcal{F}(M)$ for all $x \in M$. Finally, assume (iv) and we will prove (v). Notice that the mapping $\delta: M \rightarrow \mathcal{F}(M)$ is continuous and its range is $\mu$-essentially separable: indeed, $\mu$ is almost Radon so it is concentrated on $\operatorname{supp}(\mu)$ which is separable. Thus $\delta$ is $\mu$-measurable and, since

$$
\int_{M}\|\delta(x)\| d \mu(x)=\int_{M} \rho(x) d \mu(x)
$$

is finite, the Bochner integral in (v) exists as an element of $\mathcal{F}(M)$ (see e.g. [19, Theorems II.1.2 and II.2.2]), and its action on each $f \in \operatorname{Lip}_{0}(M)$ is obviously the same as that of $\mathcal{L} \mu$. This finishes the proof.

Note that the implication (iv) $\Rightarrow$ (ii) is contained in [9, Proposition 2.7] without our assumptions on separability of $M$ or regularity of $\mu$. However, the proof in that paper appears to have a gap. Indeed, the authors obtain weak* continuity by combining Lebesgue's dominated convergence theorem with the Banach-Dieudonné theorem. Thus, they are implicitly applying the dominated convergence theorem to nets instead of sequences, which is not valid in general. In order to reduce the argument to sequences one needs weak* metrizability, and thus $\mu$ has to be concentrated on a separable set. This is precisely where we use our extra hypothesis. We do not know whether the implication (i) $\Rightarrow$ (ii) holds for non-separable $M$ without regularity assumptions on $\mu$, but if it does, the proof of that fact requires a more delicate argument.

Using the condition that $\rho$ (or $\rho^{\mathcal{U}}$ ) be $\mu$-integrable, we obtain the following simple consequence: if $\mathcal{L} \mu \in \mathcal{F}(M)^{* *}$ then $\mathcal{L}\left(\mu \uparrow_{E}\right) \in \mathcal{F}(M)^{* *}$ for any Borel subset $E$ of $M^{\mathcal{U}}$. If moreover $M$ is separable or $\mu$ is inner regular then $\mathcal{L}\left(\mu \uparrow_{E}\right) \in \mathcal{F}(M)$ for any Borel $E \subset M$, in particular $\mathcal{L}\left(\mu \uparrow_{M}\right) \in \mathcal{F}(M)$.

We also get the following immediate consequence:

Corollary 4.2.3. The following are equivalent:

(i) $M$ is bounded,

(ii) every Radon measure on $M$ induces an element of $\mathcal{F}(M)$,

(iii) every Radon measure on $M^{\mathcal{U}}$ induces an element of $\mathcal{F}(M)^{* *}$.

Proof. If $M$ is bounded then so are $\rho$ and $\rho^{\mathcal{U}}$, and hence $\rho^{\mathcal{U}}$ is $\mu$-integrable for every finite measure $\mu$ on $M$ or $M^{\mathcal{U}}$. On the other hand, if $M$ is unbounded then we may find points $x_{n} \in M$ such that $d\left(x_{n}, 0\right) \geq 2^{n}$, and $\mu=\sum_{n} 2^{-n} \delta_{x_{n}}$ is a finite Radon measure such that $\int_{M} \rho d \mu=\infty$. 


\section{Uniqueness of representing measures}

A natural question is whether it is possible for different measures to induce the same functional on $\operatorname{Lip}_{0}(M)$. We can prove that the representing measure must be unique (up to its content at $\{0\}$ ) without requiring finiteness; instead, we make a milder assumption of regularity. This will follow from the next result, which shows that the property of positivity and the support are common for $\mu$ and $\mathcal{L} \mu$. Note that the latter provides additional justification for our choice of the name "support" in Chapter 3.

Proposition 4.2.4. Suppose that $\phi \in \mathcal{F}(M)^{* *}$ is induced by an almost Radon measure $\mu$ on $M^{\mathcal{U}}$. Then $\mathcal{S}(\phi)=\operatorname{supp}(\mu)$, and $\phi$ is positive if and only if $\mu$ is positive.

Proof. For the rest of the proof, we fix a Hahn decomposition $A^{+}, A^{-}$of $M^{\mathcal{U}}$ associated to $\mu$. We will also consider the open sets

$$
R_{n}=\left\{\zeta \in M^{\mathcal{U}}: \rho^{\mathcal{U}}(\zeta)>2^{-n}\right\}
$$

for $n \in \mathbb{N}$. Note that $R_{n} \subset R_{n+1}$ and $\bigcup_{n \in \mathbb{N}} R_{n}=M^{\mathcal{U}} \backslash\{0\}$.

We will start with the first assertion. Let $\zeta \in M^{\mathcal{U}}$ and $\operatorname{suppose} \zeta \notin \operatorname{supp}(\mu)$. Then there is an open neighborhood $U$ of $\zeta$ such that $|\mu|(U)=0$; by passing to a smaller neighborhood, we may assume that $|\mu|\left(\bar{U}^{\mathcal{U}}\right)=0$. If $f \in \operatorname{Lip}_{0}(M)$ is supported on $U \cap M$, then $f^{\mathcal{U}}$ is supported on $\bar{U}^{\mathcal{U}}$ and $\langle f, \phi\rangle=\int_{\bar{U}^{\mathcal{U}}} f^{\mathcal{U}} d \mu=0$. Thus $\zeta \notin \mathcal{S}(\phi)$ by Proposition 3.5.6.

Now suppose $\zeta \in \operatorname{supp}(\mu)$ and let $U$ be a neighborhood of $\zeta$. We will construct a function $f \in \operatorname{Lip}_{0}(M)$ such that $\operatorname{supp}(f) \subset U \cap M$ and $\langle f, \phi\rangle>0$ and this will show that $\zeta \in \mathcal{S}(\phi)$, again by Proposition 3.5.6. Let $U^{\prime}$ be a neighborhood of $\zeta$ such that ${\overline{U^{\prime}}}^{\mathcal{U}} \subset U$, then $|\mu|\left(U^{\prime} \backslash\{0\}\right)>0$ since $\mu(\{0\})=0$. Writing $U^{\prime} \backslash\{0\}=\bigcup_{n \in \mathbb{N}}\left(U^{\prime} \cap R_{n}\right)$ as a nested union, we may find an $n \in \mathbb{N}$ such that $|\mu|\left(U^{\prime \prime}\right)>0$ where $U^{\prime \prime}=U^{\prime} \cap R_{n}$. Since $0 \notin \overline{R_{n}} \mathcal{U}$ the measure $\mu \uparrow_{R_{n}} \mathcal{U}$, hence also $\mu \uparrow_{U^{\prime \prime}}$, is Radon by assumption. Let $B^{ \pm}=U^{\prime \prime} \cap A^{ \pm}$and, using the Radonness of $\mu \uparrow_{U^{\prime \prime}}$, choose compact sets $K^{ \pm} \subset B^{ \pm}$such that $|\mu|\left(B^{+} \backslash K^{+}\right)$and $|\mu|\left(B^{-} \backslash K^{-}\right)$are less than $\frac{1}{4}|\mu|\left(U^{\prime \prime}\right)$. The compact sets $K^{+}, K^{-}$and $M^{\mathcal{U}} \backslash U^{\prime \prime}$ are pairwise disjoint, hence by Proposition 3.4.4 they have pairwise disjoint open neighborhoods $V^{+}$, $V^{-}, W$ whose intersections with $M$ are at a positive distance from each other. Therefore there exists $f \in \operatorname{Lip}_{0}(M)$ such that $|f| \leq 1, f=1$ on $V^{+} \cap M, f=-1$ on $V^{-} \cap M$, and $f=0$ on $W \cap M$. Then $\operatorname{supp}(f) \subset \overline{U^{\prime \prime} \cap M} \subset U \cap M$ and $f^{\mathcal{U}}$ vanishes outside of $U^{\prime \prime}$, and we have

$$
\langle f, \phi\rangle=\int_{M^{\mathcal{U}}} f^{\mathcal{U}} d \mu=|\mu|\left(K^{+}\right)+|\mu|\left(K^{-}\right)+\int_{U^{\prime \prime} \backslash\left(K^{+} \cup K^{-}\right)} f^{\mathcal{U}} d \mu
$$


and therefore

$$
\begin{aligned}
|\mu|\left(U^{\prime \prime}\right)-\langle f, \phi\rangle & =|\mu|\left(B^{+}\right)+|\mu|\left(B^{-}\right)-\langle f, \phi\rangle \\
& \leq|\mu|\left(B^{+} \backslash K^{+}\right)+|\mu|\left(B^{-} \backslash K^{-}\right)+|\mu|\left(U^{\prime \prime} \backslash\left(K^{+} \cup K^{-}\right)\right) \\
& =2|\mu|\left(B^{+} \backslash K^{+}\right)+2|\mu|\left(B^{-} \backslash K^{-}\right)<|\mu|\left(U^{\prime \prime}\right)
\end{aligned}
$$

thus $\langle f, \phi\rangle>0$ as required.

Now we proceed with the second assertion. If $\mu$ is positive then $\phi$ is obviously positive, too. Assume now that $\mu$ is not positive, that is $|\mu|\left(A^{-}\right)>0$. Then, as above, there exists $n \in \mathbb{N}$ such that $0<|\mu|\left(A^{-} \cap R_{n}\right)<\infty$ and that $\mu \uparrow_{R_{n}}$ is Radon. Let $B^{ \pm}=A^{ \pm} \cap R_{n}$. By inner regularity there are compact sets $K^{ \pm} \subset B^{ \pm}$such that $|\mu|\left(B^{+} \backslash K^{+}\right)$and $|\mu|\left(B^{-} \backslash K^{-}\right)$are less than $\frac{1}{3}|\mu|\left(B^{-}\right)$. Since $K^{+} \cup\left(M^{\mathcal{U}} \backslash R_{n}\right)$ and $K^{-}$are disjoint and compact, by Proposition 3.4.4 they have disjoint open neighborhoods $V^{+}, V^{-}$such that

$$
d\left(V^{+} \cap M, V^{-} \cap M\right)>0,
$$

and there exists $f \in \operatorname{Lip}_{0}(M)$ such that $0 \leq f \leq 1, f=0$ on $V^{+} \cap M$, and $f=1$ on $V^{-} \cap M$. Thus $f^{\mathcal{U}}$ vanishes outside of $R_{n}$ and

$$
\begin{aligned}
\langle f, \phi\rangle=\int_{M^{\mathcal{U}}} f^{\mathcal{U}} d \mu & =\mu\left(K^{-}\right)+\int_{R_{n}^{\mathcal{U}} \backslash\left(K^{+} \cup K^{-}\right)} f d \mu \\
& \leq-|\mu|\left(K^{-}\right)+|\mu|\left(B^{+} \backslash K^{+}\right)+|\mu|\left(B^{-} \backslash K^{-}\right) \\
& =-|\mu|\left(B^{-}\right)+|\mu|\left(B^{+} \backslash K^{+}\right)+2|\mu|\left(B^{-} \backslash K^{-}\right)<0
\end{aligned}
$$

and, since $f \geq 0$, this implies that $\phi$ is not positive.

Let us remark that the same argument is valid for $\phi \in \mathcal{F}(M)$ and measures on $M$, with the following adjustments:

- Proposition 3.3.10 is used instead of Proposition 3.5.6,

- Proposition 3.4.4 is replaced by the fact that two disjoint closed subsets $A, B$ of $M$, at least one of them compact, can also be separated by neighborhoods at a positive distance of each other.

Thus we also have:

Proposition 4.2.5. Suppose that $m \in \mathcal{F}(M)$ is induced by an almost Radon measure $\mu$ on $M$. Then $\operatorname{supp}(m)=\operatorname{supp}(\mu)$, and $m$ is positive if and only if $\mu$ is positive.

Using the equivalence of supports, we can now prove the uniqueness of the measure inducing a functional on $\operatorname{Lip}_{0}(M)$ as long as it is inner regular. 
Proposition 4.2.6. Let $\mu, \lambda$ be almost Radon measures on $M^{\mathcal{U}}$ (resp. on $\left.M\right)$, and suppose that they induce the same functional $\mathcal{L} \mu=\mathcal{L} \lambda$ in $\mathcal{F}(M)^{* *}$ (resp. in $\mathcal{F}(M)$ ). Then $\mu=\lambda$.

Proof. We will prove the statement for functionals in $\mathcal{F}(M)^{* *}$ and measures on $M^{\mathcal{U}}$, the predual case is proved with an analogous argument. For $n \in \mathbb{N}$, let $\mu_{n}, \lambda_{n}$ be the measures given by $d \mu_{n}=G_{-n}^{\mathcal{U}} d \mu$ and $d \lambda_{n}=G_{-n}^{\mathcal{U}} d \lambda$. By Lemma 4.1.2, they are Radon measures on $M^{\mathcal{U}}$ and

$$
\mathcal{L} \mu_{n}=\mathcal{L} \mu \circ W_{G_{-n}}=\mathcal{L} \lambda \circ W_{G_{-n}}=\mathcal{L} \lambda_{n}
$$

Therefore, from the linearity of the operator $\mathcal{L}$ on finite measures and from Proposition 4.2 .4 we get that

$$
\operatorname{supp}\left(\mu_{n}-\lambda_{n}\right)=\mathcal{S}\left(\mathcal{L}\left(\mu_{n}-\lambda_{n}\right)\right)=\mathcal{S}\left(\mathcal{L} \mu_{n}-\mathcal{L} \lambda_{n}\right)=\mathcal{S}(0)=\varnothing,
$$

which implies that $\mu_{n}=\lambda_{n}$ by Radonness. An application of Lemma 4.1.2(b) now yields $\mu=\lambda$ as claimed.

Let us remark that the uniqueness result in Proposition 4.2.6 for the bidual is yet another hint that the uniform compactification is really the "correct" compactification to consider when analyzing $\mathcal{F}(M)^{* *}$. Indeed, that result fails for any compactification where we can find two different elements $\zeta, \xi$ that cannot be separated by Lipschitz functions on $M$ (e.g. the Stone-Čech compactification in Example 3.4.1) as the nonzero measure $\delta_{\zeta}-\delta_{\xi}$ induces the null functional.

Something similar happens with the next theorem, which is probably the key result in this chapter. In order to motivate it, let us first mention an immediate consequence of Propositions 4.2.1 and 4.2.2: Let $\mu$ be an almost Radon measure on $M^{\mathcal{U}}$ that induces a functional in $\mathcal{F}(M)^{* *}$. If $\mu$ is concentrated on $M$, then the induced functional is actually in $\mathcal{F}(M)$. It is natural to ask whether the opposite implication also holds. That is, if $\mathcal{L} \mu \in \mathcal{F}(M)$, must $\mu$ be concentrated on $M$ ? The answer is negative if we consider any compactification that is strictly larger than $M^{\mathcal{U}}$, the simplest counterexample being again $\delta_{\zeta}-\delta_{\xi}$ as in the previous paragraph. However, the answer is positive for the uniform compactification.

Theorem 4.2.7. Let $\mu$ be an almost Radon measure on $M^{\mathcal{U}}$ and suppose that $\mathcal{L} \mu \in \mathcal{F}(M)^{* *}$. Then the following are equivalent:

(i) $\mathcal{L} \mu \in \mathcal{F}(M)$,

(ii) $\mathcal{L} \mu=\mathcal{L}\left(\mu \uparrow_{M}\right)$,

(iii) $\mu$ is concentrated on $M$.

In order to prove this theorem, we will require the following property whose proof may be found within the proof of [53, Theorem 3.43]; in that reference, the property is actually proved for elements of $\beta M$ but it is equally valid in $M^{\mathcal{U}}$. 
Lemma 4.2.8. For every $\zeta \in M^{\mathcal{U}} \backslash M$ there is $r>0$ such that, for every $p \in M$, every net in $M$ that converges to $\zeta$ is eventually disjoint from $B(p, r)$.

Proof. Fix $\zeta$ and assume that the lemma fails, then for every $n \in \mathbb{N}$ there exist $p_{n} \in M$ and a net $\left(x_{i}^{(n)}\right)$ in $M$ that converges to $\zeta$ and such that $\left(x_{i}^{(n)}\right)$ is frequently in $B\left(p_{n}, \frac{1}{n}\right)$, i.e. $f_{n}\left(x_{i}^{(n)}\right)<\frac{1}{n}$ frequently, where $f_{n}: x \mapsto d\left(x, p_{n}\right)$. Taking limits on $i$ we get $f_{n}^{\mathcal{U}}(\zeta) \leq \frac{1}{n}$. Fix $m>n \in \mathbb{N}$, then we can choose an element of the net $\left(x_{i}^{(m)}\right)$ such that $f_{n}\left(x_{i}^{(m)}\right)<\frac{2}{n}$ (because that net converges to $\zeta$ ) and moreover $x_{i}^{(m)} \in B\left(p_{m}, \frac{1}{m}\right)$, hence

$$
d\left(p_{n}, p_{m}\right) \leq d\left(p_{n}, x_{i}^{(m)}\right)+d\left(x_{i}^{(m)}, p_{m}\right)<\frac{2}{n}+\frac{1}{m}<\frac{3}{n} .
$$

It follows that the sequence $\left(p_{n}\right)$ is Cauchy, therefore it converges to some $p \in M$. Now let $U$ be any neighborhood of $\zeta$, then for every $n \in \mathbb{N}$ we may find $m \geq n$ such that $p_{m} \in B\left(p, \frac{1}{n}\right)$ and $x_{i}^{(m)} \in U$ such that $d\left(x_{i}^{(m)}, p_{m}\right)<\frac{1}{m}$, so $d(M \cap U, p) \leq$ $d\left(x_{i}^{(m)}, p\right)<\frac{2}{n}$. Thus Proposition 3.4.4 implies that $p \in U$. We conclude that $\zeta=p \in M$, a contradiction.

Proof of Theorem 4.2.7. (iii) $\Rightarrow$ (ii) is obvious and (ii) $\Rightarrow$ (i) follows from the remarks after the proof of Proposition 4.2.2.

It is enough to prove the implication (i) $\Rightarrow$ (iii) when $\mu$ is Radon. Indeed, if (iii) fails then $|\mu|\left(M^{\mathcal{U}} \backslash M\right)>0$ and Lemma 4.1 .2 implies that $\left|\mu_{n}\right|\left(M^{\mathcal{U}} \backslash M\right)>0$ for some $n \in \mathbb{N}$, where $d \mu_{n}=G_{-n}^{\mathcal{U}} d \mu$. So $\mathcal{L} \mu_{n}=\mathcal{L} \mu \circ W_{G_{-n}} \in \mathcal{F}(M)$ but $\mu_{n}$ is not concentrated on $M$. That is, (i) $\Rightarrow$ (iii) fails for the Radon measure $\mu_{n}$.

Hence, for the rest of the proof we will assume (i) and suppose that $\mu$ is Radon. Let $A^{+}, A^{-}$be a Hahn decomposition of $M^{\mathcal{U}}$ associated to $\mu$, denote $B^{ \pm}=A^{ \pm} \backslash M$, and fix $\varepsilon>0$. We will prove that $|\mu|\left(B^{ \pm}\right)<7 \varepsilon$ and this will imply (iii).

For every $p \in M$ consider the 1-Lipschitz function $\rho_{p}$ given by $\rho_{p}(x)=d(x, p)$ for $x \in M$, and define

$$
s(\zeta)=\inf \left\{\rho_{p}^{\mathcal{U}}(\zeta): p \in M\right\}
$$

for every $\zeta \in M^{\mathcal{U}}$, where each $\rho_{p}$ is extended to a continuous function with values in $[0,+\infty]$ by Proposition 3.4.5. It is clear that $s(\zeta)=0$ if $\zeta \in M$, and Lemma 4.2 .8 asserts that $s(\zeta)>0$ when $\zeta \notin M$. Thus $M^{\mathcal{U}} \backslash M=\bigcup_{n \in \mathbb{N}} \mathcal{K}_{n}$ where

$$
\mathcal{K}_{n}=\left\{\zeta \in M^{\mathcal{U}}: s(\zeta) \geq \frac{1}{n}\right\}
$$

Since $s$ is the infimum of the continuous functions $\rho_{p}^{\mathcal{U}}$, it is an upper semicontinuous function on $M^{\mathcal{U}}$ and so each $\mathcal{K}_{n}$ is compact. We have then that $\mu\left(B^{ \pm}\right)=$ $\lim _{n \rightarrow \infty} \mu\left(B^{ \pm} \cap \mathcal{K}_{n}\right)$. Choose $n \in \mathbb{N}$ such that $|\mu|\left(B^{ \pm} \backslash \mathcal{K}_{n}\right)<\varepsilon$. By inner regularity 
of $\mu$ we may choose compact sets $K^{ \pm} \subset B^{ \pm} \cap \mathcal{K}_{n}$ such that $|\mu|\left(\left(B^{ \pm} \cap \mathcal{K}_{n}\right) \backslash K^{ \pm}\right)<\varepsilon$, and hence $|\mu|\left(B^{ \pm} \backslash K^{ \pm}\right)<2 \varepsilon$. Since $K^{+}$and $K^{-}$are disjoint, by Proposition 3.4.4 there are disjoint open neighborhoods $U^{ \pm}$of $K^{ \pm}$such that $d\left(U^{+} \cap M, U^{-} \cap M\right)>0$. Denote

$$
r=\frac{1}{2} \min \left\{\frac{1}{n}, d\left(U^{+} \cap M, U^{-} \cap M\right)\right\} .
$$

Now let $\mathfrak{F}$ be the family of all finite subsets of $M$ containing 0 . For every $F \in \mathfrak{F}$, let

$$
f_{F}(x)=1 \wedge \frac{1}{r} d\left(x, F \cup\left(U^{-} \cap M\right)\right)
$$

for $x \in M$. Then $f_{F} \in \operatorname{Lip}_{0}(M)$ is such that $0 \leq f_{F} \leq 1,\left\|f_{F}\right\|_{L} \leq \frac{1}{r}$, and $f_{F}=0$ on $U^{-} \cap M$, hence $f_{F}^{\mathcal{U}}=0$ on $K^{-}$. We claim that $f_{F}^{\mathcal{U}}=1$ on $K^{+}$. Indeed, let $\zeta \in K^{+}$and choose a net $\left(x_{i}\right)$ in $M$ that converges to $\zeta$. We may assume that $x_{i} \in U^{+}$for all $i$ by passing to a subnet, so that $d\left(x_{i}, U^{-} \cap M\right)>r$. Since $s(\zeta) \geq \frac{1}{n}>r$ (because $\zeta \in \mathcal{K}_{n}$ ) and $F$ is finite, we may pass to a further subnet such that $d\left(x_{i}, p\right)=\rho_{p}\left(x_{i}\right)>r$ for every $p \in F$. Therefore $d\left(x_{i}, F \cup\left(U^{-} \cap M\right)\right)>r$ and $f_{F}\left(x_{i}\right)=1$ for all $i$, proving the claim.

Consider the net $\left(f_{F}\right)_{F \in \mathfrak{F}}$ in $\operatorname{Lip}_{0}(M)$, where $\mathfrak{F}$ is directed by inclusion. It is a norm-bounded net and it converges pointwise (even monotonically) to 0, as for any fixed $x \in M$ we have $f_{F}(x)=0$ whenever $F \supset\{x\}$. Hence $f_{F} \stackrel{w^{*}}{\rightarrow} 0$. Since we assume $\mathcal{L} \mu \in \mathcal{F}(M)$, we have $\left\langle f_{F}, \mathcal{L} \mu\right\rangle \rightarrow 0$. But $\mathcal{L}\left(\mu \uparrow_{M}\right) \in \mathcal{F}(M)$, so $\left\langle f_{F}, \mathcal{L}\left(\mu \uparrow_{M}\right)\right\rangle \rightarrow 0$ as well. Choose $F \in \mathfrak{F}$ such that $\left|\left\langle f_{F}, \mathcal{L} \mu-\mathcal{L}\left(\mu \uparrow_{M}\right)\right\rangle\right|<\varepsilon$. Then

$$
\begin{aligned}
\left\langle f_{F}, \mathcal{L} \mu-\mathcal{L}\left(\mu \uparrow_{M}\right)\right\rangle & =\int_{M^{\mathcal{U}} \backslash M} f_{F}^{\mathcal{U}} d \mu \\
& =\mu\left(K^{+}\right)+\int_{B^{+} \backslash K^{+}} f_{F}^{\mathcal{U}} d \mu+\int_{B^{-} \backslash K^{-}} f_{F}^{\mathcal{U}} d \mu
\end{aligned}
$$

so that

$$
\mu\left(K^{+}\right)<\varepsilon+|\mu|\left(B^{+} \backslash K^{+}\right)+|\mu|\left(B^{-} \backslash K^{-}\right)<5 \varepsilon
$$

and therefore $\mu\left(B^{+}\right)=\mu\left(B^{+} \backslash K^{+}\right)+\mu\left(K^{+}\right)<7 \varepsilon$. A similar construction replacing $U^{-}$with $U^{+}$in (4.1) shows that $\left|\mu\left(B^{-}\right)\right|<7 \varepsilon$. This finishes the proof.

Note that the net $\left(f_{F}\right)$ used in the proof of Theorem 4.2.7 is pointwise decreasing, so we have actually proved the following formally stronger result: if $\mathcal{L} \mu$ is a normal functional, then $\mu$ is concentrated on $M$. Of course, this is in fact an equivalent result due to Theorem 3.2.5. 


\section{Functionals that admit an integral representation}

To end this section, we now proceed in the opposite direction to Propositions 4.2.1 and 4.2.2 and attempt to identify which functionals in $\mathcal{F}(M)$ and $\mathcal{F}(M)^{* *}$ can be represented by Radon measures. Let us begin with a simple observation:

Lemma 4.2.9. Suppose that $\phi \in \mathcal{F}(M)^{* *}$ is induced by a Borel measure on $M^{\mathcal{U}}$. Then $\phi$ avoids 0 and infinity.

Proof. Let $\phi=\mathcal{L} \mu$ where $\mu$ is a Borel measure on $M^{\mathcal{U}}$. Then $\mathcal{L} \mu^{+}, \mathcal{L} \mu^{-} \in \mathcal{F}(M)^{* *}$ by the remarks after Proposition 4.2.2. To show that $\phi$ avoids 0 and infinity, it is enough to prove that $\phi \circ W_{\Pi_{n}} \stackrel{w^{*}}{\longrightarrow} \phi$, i.e. that $\left\langle f \Pi_{n}, \phi\right\rangle \rightarrow\langle f, \phi\rangle$ for every $f \in \operatorname{Lip}_{0}(M)$. Now notice that

$$
\begin{aligned}
\lim _{n \rightarrow \infty}\left\langle f^{+} \Pi_{n}, \mathcal{L} \mu^{+}\right\rangle & =\lim _{n \rightarrow \infty} \int_{M^{\mathcal{R}}}\left(f^{+} \Pi_{n}\right)^{\mathcal{U}} d \mu^{+} \\
& =\lim _{n \rightarrow \infty} \int_{M^{\mathcal{R}}}\left(f^{+}\right)^{\mathcal{U}} \Pi_{n}^{\mathcal{U}} d \mu^{+}=\int_{M^{\mathcal{R}}}\left(f^{+}\right)^{\mathcal{U}} d \mu^{+}=\left\langle f^{+}, \mathcal{L} \mu^{+}\right\rangle
\end{aligned}
$$

by Lebesgue's monotone convergence theorem because $\Pi_{n}^{\mathcal{U}}$ converges pointwise and monotonically to $\chi_{M^{\mathcal{R}} \backslash\{0\}}$. Note that we have used the fact that $\mu$ is concentrated on $M^{\mathcal{R}}$ by Proposition 4.2.1. By replacing $f^{+}$with $f^{-}$and/or $\mu^{+}$with $\mu^{-}$we get the desired conclusion.

Lemma 4.2.9 is evidently not a characterization of functionals induced by measures (consider e.g. derivations). The next two theorems will provide such characterizations, although some of their implications only hold under assumptions of boundedness or positivity. In order to state them, let us introduce the following notation: if $m \in \operatorname{span} \delta(M)$ is a finitely supported element of $\mathcal{F}(M)$, say $m=\sum_{k=1}^{n} a_{k} \delta\left(x_{k}\right)$ for distinct $x_{k} \in M \backslash\{0\}$, let

$$
\|m\|_{1}=\sum_{k=1}^{n}\left|a_{k}\right| .
$$

It is clear that this value is uniquely defined, and that $m=\mathcal{L} \mu$ where $\mu=$ $\sum_{k=1}^{n} a_{k} \delta_{x_{k}} \in \mathcal{M}_{0}(M)$ satisfies $\|\mu\|=\|m\|_{1}$.

Theorem 4.2.10. Let $\phi \in \mathcal{F}(M)^{* *}$. If $\phi$ is induced by a Radon measure on $M^{\mathcal{U}}$, then it is the weak* limit of a net $\left(m_{i}\right)$ of elements of $\operatorname{span} \delta(M)$ such that $\left\|m_{i}\right\|_{1}$ is bounded. If $\phi$ avoids infinity strongly, or if it is positive and avoids infinity, then the converse also holds. Moreover, if $\phi$ is positive then the $\left(m_{i}\right)$ can be chosen to be positive.

Proof. Suppose that $\phi=\mathcal{L} \mu \in \mathcal{F}(M)^{* *}$ for some $\mu \in \mathcal{M}\left(M^{\mathcal{U}}\right)$, and assume $\|\mu\| \leq 1$ without loss of generality. We claim that $\phi \in \overline{\operatorname{conv}} w^{*}( \pm \delta(M))$, which 
will prove the forward implication with the bound $\left\|m_{i}\right\|_{1} \leq 1$. Indeed, by the Krein-Milman theorem we have

$$
\mu \in B_{\mathcal{M}\left(M^{\mathcal{U}}\right)}=\overline{\operatorname{conv}}^{w^{*}} \operatorname{ext} B_{\mathcal{M}\left(M^{\mathcal{U}}\right)}=\overline{\operatorname{conv}}^{w^{*}}\left\{ \pm \delta_{\zeta}: \zeta \in M^{\mathcal{U}}\right\}
$$

Now notice that if $\left(x_{i}\right)$ is a net in $M$ that converges to $\zeta \in M^{\mathcal{U}}$ then $\delta_{x_{i}} \stackrel{w^{*}}{\longrightarrow} \delta_{\zeta}$ in $\mathcal{M}\left(M^{\mathcal{U}}\right)$. We conclude that $\mu \in \bar{A}^{w^{*}}$ where $A=\operatorname{conv}\left\{ \pm \delta_{x}: x \in M\right\}$, hence there is a net $\left(\mu_{i}\right)$ in $A$ that converges weak* to $\mu$. This implies in particular that $\left\langle f, \mathcal{L} \mu_{i}\right\rangle \rightarrow\langle f, \mathcal{L} \mu\rangle=\langle f, \phi\rangle$ for any bounded $f \in \operatorname{Lip}_{0}(M)$. It is clear that $m_{i}=\mathcal{L} \mu_{i} \in \operatorname{conv}( \pm \delta(M))$ and that $\left\|m_{i}\right\|_{1}=\left\|\mu_{i}\right\| \leq 1$. Now notice that, for any $f \in \operatorname{Lip}_{0}(M)$ and $n \in \mathbb{N}, f H_{n}$ is a bounded Lipschitz function and therefore

$$
\lim _{i}\left\langle m_{i} \circ W_{H_{n}}, f\right\rangle=\lim _{i}\left\langle m_{i}, f H_{n}\right\rangle=\left\langle f H_{n}, \phi\right\rangle=\left\langle f, \phi \circ W_{H_{n}}\right\rangle
$$

i.e. $m_{i} \circ W_{H_{n}} \stackrel{w^{*}}{\longrightarrow} \phi \circ W_{H_{n_{*}}}$. But clearly $m_{i} \circ W_{H_{n}} \in \operatorname{conv}( \pm \delta(M))$ for all $i$ and $n$, hence $\phi \circ W_{H_{n}} \in \overline{\mathrm{conv}}^{w^{*}}( \pm \delta(M))$. To finish, notice that $\phi$ avoids infinity by Lemma 4.2.9, hence $\phi \circ W_{H_{n}} \rightarrow \phi$ and $\phi \in \overline{\operatorname{conv}} w^{*}( \pm \delta(M))$.

If $\phi$ is positive in the argument above, then $\mu$ may be chosen to be positive by Proposition 4.2.4, and we can apply the Krein-Milman theorem to the positive unit ball $B_{\mathcal{M}\left(M^{\mathcal{U}}\right)}^{+}$instead of $B_{\mathcal{M}\left(M^{\mathcal{U}}\right)}$, which is a $w^{*}$-compact convex set whose extreme points are 0 and $+\delta_{\zeta}$ for $\zeta \in M^{\mathcal{U}}$. We then get that $\phi \in \overline{\mathrm{conv}} w^{*} \delta(M)$.

For the converse implication, let $\phi \in \mathcal{F}(M)^{* *}$ be the weak ${ }^{*}$ limit of finitely supported elements $m_{i}$ of $\mathcal{F}(M)$ such that $\left\|m_{i}\right\|_{1} \leq 1$ for all $i$. Then $m_{i}=\mathcal{L} \mu_{i}$ where $\mu_{i} \in \mathcal{M}\left(M^{\mathcal{U}}\right)$ has finite support and $\left\|\mu_{i}\right\|=\left\|m_{i}\right\|_{1} \leq 1$. Since $B_{\mathcal{M}\left(M^{\mathcal{U}}\right)}=B_{C\left(M^{\mathcal{U}}\right)^{*}}$ is $w^{*}$-compact, we may replace $\left(\mu_{i}\right)$ with a subnet that converges weak* to some $\mu \in \mathcal{M}\left(M^{\mathcal{U}}\right)$, that is, such that $\lim _{i} \int_{M^{\mathcal{U}}} g d \mu_{i}=\int_{M^{\mathcal{U}}} g d \mu$ for each $g \in C\left(M^{\mathcal{U}}\right)$. In particular, if $f \in \operatorname{Lip}_{0}(M)$ is bounded then we have

$$
\int_{M^{\mathcal{U}}} f^{\mathcal{U}} d \mu=\lim _{i} \int_{M^{\mathcal{U}}} f^{\mathcal{U}} d \mu_{i}=\lim _{i}\left\langle f, m_{i}\right\rangle=\langle f, \phi\rangle .
$$

Suppose first that $\phi$ avoids infinity strongly, so $\phi=\phi \circ W_{H_{n}}$ for some $n \in \mathbb{N}$. Then $f H_{n}$ is bounded for every $f \in \operatorname{Lip}_{0}(M)$, so we have

$$
\langle f, \phi\rangle=\left\langle f, \phi \circ W_{H_{n}}\right\rangle=\left\langle f H_{n}, \phi\right\rangle=\int_{M^{\mathcal{U}}}\left(f H_{n}\right)^{\mathcal{U}} d \mu=\int_{M^{\mathcal{U}}} f^{\mathcal{U}} d \lambda
$$

where $d \lambda=H_{n}^{\mathcal{U}} d \mu$ defines a Borel measure on $M^{\mathcal{U}}$. Note that $|\lambda|(E) \leq|\mu|(E)$ for any Borel set $E \subset M^{\mathcal{U}}$, therefore $\lambda$ is finite and its regularity follows easily from that of $\mu$. Thus $\lambda \in \mathcal{M}\left(M^{\mathcal{U}}\right)$ and $\phi=\mathcal{L} \lambda$ as required.

Now assume that $\phi$ is positive and avoids infinity, i.e. $\phi=\lim _{n} \phi \circ W_{H_{n}}$. For any function $f \in \operatorname{Lip}_{0}(M)$ and any $n$, the function $f H_{n}$ is bounded and $\left(f H_{n}\right)^{\mathcal{U}}=0$ on 
$M^{\mathcal{U}} \backslash M^{\mathcal{R}}$. Moreover, $H_{n}^{\mathcal{U}}$ converges pointwise and increasingly $\chi_{M^{\mathcal{R}}}$. Therefore for a positive $f \in \operatorname{Lip}_{0}(M)$ we have

$$
\begin{aligned}
\langle f, \phi\rangle & =\lim _{n}\left\langle f, \phi \circ W_{H_{n}}\right\rangle=\lim _{n}\left\langle f H_{n}, \phi\right\rangle=\lim _{n} \int_{M^{\mathcal{U}}}\left(f H_{n}\right)^{\mathcal{U}} d \mu \\
& =\lim _{n} \int_{M^{\mathcal{R}}}\left(f H_{n}\right)^{\mathcal{U}} d \mu=\lim _{n} \int_{M^{\mathcal{R}}} f^{\mathcal{U}} H_{n}^{\mathcal{U}} d \mu=\int_{M^{\mathcal{R}}} f^{\mathcal{U}} d \mu=\int_{M^{\mathcal{U}}} f^{\mathcal{U}} d\left(\mu\left\lceil_{M^{\mathcal{R}}}\right)\right.
\end{aligned}
$$

by Lebesgue's monotone convergence theorem. Decomposing any $f \in \operatorname{Lip}_{0}(M)$ as $f=f^{+}-f^{-}$, we may conclude that $\phi=\mathcal{L} \mu \uparrow_{M^{\mathcal{R}}}$. Obviously $\mu \uparrow_{M^{\mathcal{R}}} \in \mathcal{M}\left(M^{\mathcal{U}}\right)$ since $\mu \in \mathcal{M}\left(M^{\mathcal{U}}\right)$.

To conclude, let us remark that $\mu$, and hence $\lambda$ (for $\phi$ that avoids infinity strongly) or $\mu \uparrow_{M^{\mathcal{R}}}$ (for positive $\phi$ that avoids infinity) are positive if all $\mu_{i}$ are.

The converse of Theorem 4.2.10 does not hold in general. We have proved that there is a measure $\mu$ whose action coincides with that of $\phi$ on all bounded functions $f \in \operatorname{Lip}_{0}(M)$, but it might differ for unbounded $f$ when $\phi$ is not assumed to avoid infinity. The following shows that we can find a counterexample whenever $M$ is unbounded:

Example 4.2.11. Suppose that $M$ is unbounded. Let $\left(x_{n}\right)$ be a sequence in $M$ such that $d\left(x_{n}, 0\right) \rightarrow \infty$ and define $m_{n}=\delta\left(x_{n}\right) / d\left(x_{n}, 0\right)$. Then $\left\|m_{n}\right\|=1$, so there is a subnet $\left(m_{n_{i}}\right)$ that converges weak* to some $\phi \in \mathcal{F}(M)^{* *}$ which is clearly positive. We claim that $\phi$ cannot be represented by a positive Borel measure $\mu$ on $M^{\mathcal{U}}$, even if we allow it to be $\sigma$-finite. Suppose otherwise, then we may also assume that $\mu(\{0\})=0$ and Proposition 4.2.1 implies that $\mu$ is concentrated on $M^{\mathcal{R}}$. Since $M^{\mathcal{R}}=\bigcup_{n=1}^{\infty} B_{n}$ where $B_{n}=\overline{B(0, n)}^{\mathcal{U}}$, we have $\mu\left(M^{\mathcal{R}}\right)=\lim _{n} \mu\left(B_{n}\right)$. Let $\rho_{n}=\rho \wedge n$, then

$$
\int_{B_{n}} \rho^{\mathcal{U}} d \mu=\int_{B_{n}} \rho_{n}^{\mathcal{U}} d \mu \leq \int_{M^{\mathcal{U}}} \rho_{n}^{\mathcal{U}} d \mu=\left\langle\rho_{n}, \phi\right\rangle=\lim _{i} \frac{\rho_{n}\left(x_{n_{i}}\right)}{d\left(x_{n_{i}}, 0\right)}=0
$$

which implies that $\mu\left(B_{n}\right)=0$ for all $n$. Thus $\mu=0$ and so $\phi=0$, but this contradicts the fact that $\langle\rho, \phi\rangle=1$.

So $\phi$ is not induced by a measure even if $\left\|m_{n}\right\|_{1}=1 / d\left(x_{n}, 0\right)$ converges to 0 . The argument in the proof of Theorem 4.2.10 still yields a measure $\mu$ such that the actions of $\phi$ and $\mu$ agree on bounded functions of $\operatorname{Lip}_{0}(M)$ : it is just $\mu=0$.

For elements of $\mathcal{F}(M)$ we have a similar result:

Theorem 4.2.12. Let $\phi \in \mathcal{F}(M)$. If $\phi$ is induced by a Radon measure on $M$, then it is the limit of a sequence $\left(m_{n}\right)$ of elements of $\operatorname{span} \delta(M)$ such that $\left\|m_{n}\right\|_{1}$ is bounded. If $\operatorname{supp}(\phi)$ is bounded or $\phi$ is positive, then the converse also holds. Moreover, if $\phi$ is positive then the $\left(m_{n}\right)$ can be chosen to be positive. 
Proof. Assume that $\phi=\mathcal{L} \mu \in \mathcal{F}(M)$ for some $\mu \in \mathcal{M}(M)$, and identify $\mu$ with an element of $\mathcal{M}\left(M^{\mathcal{U}}\right)$ that is concentrated on $M$. Theorem 4.2.10 then yields a net $\left(v_{i}\right)$ in span $\delta(M)$ that converges to $\phi$ in $\left(\mathcal{F}(M)^{* *}, w^{*}\right)$, or equivalently in $(\mathcal{F}(M), w)$, and such that $\left\|v_{i}\right\|_{1}$ is bounded. By Mazur's lemma, $\phi$ is the limit of a sequence $\left(m_{n}\right)$ of convex combinations of the $v_{i}$. Clearly $\left\|m_{n}\right\|_{1}$ is bounded by the same value as $\left\|v_{i}\right\|_{1}$, and the $m_{n}$ are positive if all $v_{i}$ are.

For the converse implication, notice that Theorem 4.2 .10 yields a measure $\mu \in$ $\mathcal{M}\left(M^{\mathcal{U}}\right)$ such that $\phi=\mathcal{L} \mu$ : indeed, every $\phi \in \mathcal{F}(M)$ avoids infinity, and it avoids infinity strongly when its support is bounded. Moreover $\mu$ is positive if the $m_{n}$ are. But $\phi \in \mathcal{F}(M)$, so the measure has to be concentrated on $M$ by Theorem 4.2.7 and therefore can be regarded as a Radon measure on $M$.

Note that in Theorems 4.2.10 and 4.2.12 the norm $\|\mu\|$ of the representing measure $\mu$ is related to the sum $\|m\|_{1}$ of the coefficients of the approximating elements of finite support. So we get the following, more succinct characterization:

Corollary 4.2.13. The set of elements of $\mathcal{F}(M)$ that can be represented by a measure in $B_{\mathcal{M}(M)}^{+}$is precisely $\overline{\operatorname{conv}} \delta(M)$. If $M$ is bounded, then the set of elements of $\mathcal{F}(M)^{* *}$ that can be represented by a measure in $B_{\mathcal{M}\left(M^{\mathcal{U}}\right)}^{+}$is precisely $\overline{\mathrm{conv}}^{w^{*}} \delta(M)$.

The next example shows that boundedness or positivity are again essential for the converse to hold in Theorem 4.2.12. In particular, it also shows that the converse in Theorem 4.2.10 does not hold if $\phi$ avoids infinity but is not positive.

Example 4.2.14. Consider the space $M$ from Example 3.3.15, i.e.

$$
M=\left\{0, x_{1}, y_{1}, x_{2}, y_{2}, \ldots\right\} \subset c_{0}
$$

where $x_{n}=2^{n} e_{n}$ and $y_{n}=\left(2^{n}+1\right) e_{n}$, and let

$$
m_{n}=\sum_{k=1}^{n} 2^{-k}\left(\delta\left(x_{k}\right)-\delta\left(y_{k}\right)\right) .
$$

Since $\left\|\delta\left(x_{n}\right)-\delta\left(y_{n}\right)\right\|=d\left(x_{n}, y_{n}\right)=1$, the sequence $\left(m_{n}\right)$ is Cauchy and converges to $m \in \mathcal{F}(M)$. Moreover $\left\|m_{n}\right\|_{1}<2$ for every $n$. Nevertheless, $m$ cannot be represented by a Radon measure on $M$. Indeed, suppose $m=\mathcal{L} \mu$ where $\mu \in$ $\mathcal{M}_{0}(M)$, then $\mu$ is supported on $\operatorname{supp}(m)=M \backslash\{0\}$ by Proposition 4.2.5, and it is clear that every $x_{n}$ belongs to the support of $\mu^{+}$. Denote $X_{n}=\left\{x_{1}, \ldots, x_{n}\right\}$, then

$$
\int_{M} \rho d|\mu| \geq \int_{X_{n}} \rho d|\mu|=\sum_{k=1}^{n} 2^{-k} \rho\left(x_{k}\right)=n
$$

for every $n$, which contradicts Proposition 4.2.2. 


\subsection{Majorizable functionals}

In this section, we will study the majorizable elements of $\mathcal{F}(M)$ and $\mathcal{F}(M)^{* *}$. Recall that a functional in $\mathcal{F}(M)$ and $\mathcal{F}(M)^{* *}$ is majorizable if it can be expressed as the difference between two positive functionals, and that not all functionals are majorizable in general but all finitely supported elements of $\mathcal{F}(M)$ are.

We shall first tackle the problem of characterizing the majorizable elements of $\mathcal{F}(M)$ and $\mathcal{F}(M)^{* *}$. Perhaps surprisingly, the results from the last section will be enough to accomplish that, as there turns out to be a very strong relationship between majorizable functionals and integral representations. The cases for $\mathcal{F}(M)$ and $\mathcal{F}(M)^{* *}$ require similar reasoning so we shall handle them more or less simultaneously, as was done in the previous section.

Let us start by observing that any element $\phi \in \mathcal{F}(M)$ that is induced by a Borel measure on $M$ is majorizable. Indeed, suppose $\phi=\mathcal{L} \mu$, then $|\mu|$ also induces an element of $\mathcal{F}(M)$ by Proposition 4.2 .2 , therefore $\phi$ is majorized by $\mathcal{L}(|\mu|)$. A similar argument applies in $\mathcal{F}(M)^{* *}$, and in that case we moreover know that $\phi$ avoids 0 and infinity by Lemma 4.2.9.

It turns out that the converse of this observation is "almost" true. We may already prove that it holds under the additional hypothesis that the avoidance of 0 is strong:

Theorem 4.3.1. Suppose that $\phi \in \mathcal{F}(M)^{* *}$ avoids infinity and $0 \notin \mathcal{S}(\phi)$. Then $\phi$ is majorizable in $\mathcal{F}(M)^{* *}$ if and only if it is induced by a Radon measure on $M^{\mathcal{U}}$.

Proof. We have already discussed the easy implication. For the other one, recall that $\phi$ avoids 0 strongly by Proposition 3.5.18(b). Assume that $\phi=\phi^{+}-\phi^{-}$where $\phi^{+}, \phi^{-} \in \mathcal{F}(M)^{* *}$ are positive. Using the notation of Corollary 3.5.4 we have

$$
\phi=\phi_{A}=\lim _{n} \phi \circ W_{H_{n}}=\lim _{n} \phi^{+} \circ W_{H_{n}}-\lim _{n} \phi^{-} \circ W_{H_{n}}=\left(\phi^{+}\right)_{A}-\left(\phi^{-}\right)_{A}
$$

so, by replacing $\phi^{ \pm}$with $\left(\phi^{ \pm}\right)_{A}$, we may assume that they avoid infinity. Similarly we may assume that they avoid 0 strongly, i.e. that $\phi^{ \pm}=\phi^{ \pm} \circ W_{G_{-n}}$ for some $n \in \mathbb{N}$.

By Proposition 2.3.4, there is a net $\left(m_{i}\right)$ that converges weak* to $\phi^{+}$and such that every $m_{i}$ is of the form

$$
m_{i}=\sum_{k=1}^{N} a_{k} \delta\left(x_{k}\right)
$$


for some $N \in \mathbb{N}, a_{k}>0$ and $x_{k} \in M$. Thus $m_{i} \circ W_{G_{-n}} \stackrel{w^{*}}{\longrightarrow} \phi^{+} \circ W_{G_{-n}}=\phi^{+}$. Each $m_{i} \circ W_{G_{-n}}$ is positive, so by Proposition 2.3.3

$$
\lim _{i}\left\|m_{i} \circ W_{G_{-n}}\right\|=\lim _{i}\left\langle\rho, m_{i} \circ W_{G_{-n}}\right\rangle=\left\langle\rho, \phi^{+}\right\rangle=\left\|\phi^{+}\right\|,
$$

hence we may assume that $\left\|m_{i} \circ W_{G_{-n}}\right\|$ is bounded. Now notice that $G_{-n}(x)=0$ for $x \in B\left(0,2^{-n}\right)$ and therefore, using again Proposition 2.3.3

$$
\begin{aligned}
\left\|m_{i} \circ W_{G_{-n}}\right\|=\left\langle\rho, m_{i} \circ W_{G_{-n}}\right\rangle & =\sum_{k=1}^{N} a_{k} G_{-n}\left(x_{k}\right) d\left(x_{k}, 0\right) \\
& \geq 2^{-n} \sum_{k=1}^{N} a_{k} G_{-n}\left(x_{k}\right)=2^{-n}\left\|m_{i} \circ W_{G_{-n}}\right\|_{1}
\end{aligned}
$$

so $\left\|m_{i} \circ W_{G_{-n}}\right\|_{1}$ is bounded. Since $\phi^{+}$is positive and avoids infinity, we may apply Theorem 4.2.10 to conclude that $\phi^{+}$is represented by a positive Radon measure $\mu^{+}$on $M^{\mathcal{U}}$.

The same argument shows that $\phi^{-}$is represented by a positive Radon measure $\mu^{-}$. Thus $\phi$ is represented by $\mu=\mu^{+}-\mu^{-} \in \mathcal{M}\left(M^{\mathcal{U}}\right)$ and this ends the proof.

In particular, if $M$ is bounded then the result applies to all $\phi$ such that $0 \notin \mathcal{S}(\phi)$. Notice that the hypothesis that $\phi$ avoids infinity cannot be omitted, as witnessed by the positive element constructed in Example 4.2.11.

A similar argument yields the corresponding result for $\mathcal{F}(M)$, but we can also obtain it as an immediate consequence by combining Theorem 4.3.1 with Theorem 4.2.7, as any element of $\mathcal{F}(M)$ avoids infinity. More than that, this also yields immediately the equivalence of majorizability in $\mathcal{F}(M)$ and in $\mathcal{F}(M)^{* *}$ under the additional assumption that the support does not contain the base point.

Theorem 4.3.2. Let $m \in \mathcal{F}(M)$ be such that $0 \notin \operatorname{supp}(m)$. Then the following are equivalent:

(i) $m$ is majorizable in $\mathcal{F}(M)$,

(ii) $m$ is majorizable in $\mathcal{F}(M)^{* *}$,

(iii) $m$ is induced by a Radon measure on $M$.

Theorem 4.3.2 does not hold (and therefore, neither does Theorem 4.3.1) for the case where the base point is contained in the (extended) support of a positive element, as showcased by the following example.

Example 4.3.3. Assume that the base point of $M$ is not isolated and choose $x_{n} \in M \backslash\{0\}$ such that $d\left(x_{n}, 0\right)<2^{-n}$ and $d\left(x_{n+1}, 0\right)<d\left(x_{n}, 0\right)$ for every $n \in \mathbb{N}$. 
Consider $m=\sum_{n=1}^{\infty} \delta\left(x_{n}\right)$, which is clearly an element of $\mathcal{F}(M)^{+}$by absolute convergence. Suppose that $m$ is represented by a Radon measure $\mu$ on $M$. We may assume that $\mu \in \mathcal{M}_{0}(M)$ and so $\mu$ is positive by Proposition 4.2.5. Let $g_{n}$ be a Lipschitz function on $\mathbb{R}$ with $0 \leq g_{n} \leq 1, g_{n}(t)=0$ for $t \leq d\left(x_{n+1}, 0\right)$ and $g_{n}(t)=1$ for $t \geq d\left(x_{n}, 0\right)$. Then

$$
\|\mu\|=\int_{M} d \mu \geq \int_{M}\left(g_{n} \circ \rho\right) d \mu=\left\langle m, g_{n} \circ \rho\right\rangle=n
$$

for any $n \in \mathbb{N}$, therefore $\mu$ is not finite, a contradiction. Note however that $\mu$ is $\sigma$-finite.

The construction in the preceding example immediately provides the following equivalence:

Proposition 4.3.4. The following are equivalent:

(i) the base point is an isolated point of $M$,

(ii) every majorizable element of $\mathcal{F}(M)$ can be represented by a Radon measure on $M$,

(iii) every majorizable element of $\mathcal{F}(M)^{* *}$ that avoids infinity can be represented by a Radon measure on $M^{\mathcal{U}}$.

We shall now extend Theorem 4.3.1 to all positive elements of $\mathcal{F}(M)^{* *}$ that avoid 0 and infinity and show that they can also be represented by a positive measure. It may not be finite, but it will always be almost Radon; recall that this implies that it is inner regular, $\sigma$-finite, and its restriction to every closed subset separated from 0 is Radon. We will construct the desired measure as an inverse limit of Radon measures that are supported away from the base point, reversing the construction from Lemma 4.1.2.

Theorem 4.3.5. Suppose that $\phi \in \mathcal{F}(M)^{* *}$ is positive and avoids 0 and infinity. Then $\phi$ is represented by a positive, almost Radon measure on $M^{\mathcal{U}}$.

Proof. For $n \in \mathbb{N}$ denote

$$
A_{n}=\left\{\zeta \in M^{\mathcal{U}}: 2^{-n} \leq \rho^{\mathcal{U}}(\zeta)\right\}
$$

and $\phi_{n}=\phi \circ W_{G_{-n}}$. Since $\phi$ avoids 0 , we have $\phi=\lim _{n} \phi_{n}$. Note that $\mathcal{S}\left(\phi_{n}\right) \subset A_{n}$ by Corollary 3.5.11, so $0 \notin \mathcal{S}\left(\phi_{n}\right)$ and, by Theorem 4.3 .1 , each $\phi_{n}$ is induced by a Radon measure $\mu_{n} \in \mathcal{M}_{0}\left(M^{\mathcal{U}}\right)$. Proposition 4.2.4 then shows that $\operatorname{supp}\left(\mu_{n}\right) \subset A_{n}$, and in particular

$$
\mu_{n}(E)=0 \text { for every Borel set } E \subset M^{\mathcal{U}} \backslash A_{n} .
$$


We also have

$$
\mathcal{L}\left(\mu_{n+1}-\mu_{n}\right)=\phi_{n+1}-\phi_{n}=\phi \circ W_{G_{-(n+1)}-G_{-n}} .
$$

Since $G_{-(n+1)}-G_{-n}=\Lambda_{-n} \geq 0$, this is a positive element of $\mathcal{F}(M)^{* *}$ and so $\mu_{n+1}-\mu_{n} \geq 0$ by Proposition 4.2.4. That is,

$$
\mu_{n+1}(E) \geq \mu_{n}(E) \text { for every Borel set } E \subset M^{\mathcal{U}} \text {. }
$$

Moreover, combining Proposition 4.2.4 and Corollary 3.5.11

$$
\operatorname{supp}\left(\mu_{n+1}-\mu_{n}\right)=\mathcal{S}\left(\phi_{n+1}-\phi_{n}\right) \subset \overline{\operatorname{supp}\left(\Lambda_{-n}\right)} \mathcal{U}
$$

hence in particular we get

$$
\mu_{n+1}(E)=\mu_{n}(E) \text { for every Borel set } E \subset A_{n-2} .
$$

By (4.3), the limit

$$
\mu(E)=\lim _{n \rightarrow \infty} \mu_{n}(E)
$$

exists and is positive (possibly infinite) for every Borel set $E \subset M^{\mathcal{U}}$. It is straightforward to check that $\mu$ is a Borel measure on $M^{\mathcal{U}}$. Notice also that $\mu(\{0\})=$ $\mu\left(M^{\mathcal{U}} \backslash M^{\mathcal{R}}\right)=0$ by Proposition 4.2.1. Moreover, (4.4) implies $\mu \uparrow_{A_{n}}=\mu_{n+2} \Upsilon_{A_{n}}$ and so

$$
\mu\left(A_{n}\right)=\mu_{n+2}\left(A_{n}\right) \leq\left\|\mu_{n+2}\right\|<\infty
$$

for every $n$. Thus, if $K$ is any closed subset of $M^{\mathcal{U}}$ such that $0 \notin K$, we have $K \subset A_{n}$ for some $n$ and therefore $\mu(K) \leq\left\|\mu_{n+2}\right\|<\infty$, so $\mu \uparrow_{K}$ is finite and regular. Hence $\mu$ is almost Radon.

We will now finish the proof by showing that $\phi$ is represented by $\mu$. Let $f \in$ $\operatorname{Lip}_{0}(M)$ be positive. Since $\phi$ avoids 0 and $W_{G_{-n}} \circ W_{G_{-(n+2)}}=W_{G_{-n}}$, we may write

$$
\begin{aligned}
\langle f, \phi\rangle & =\lim _{n \rightarrow \infty}\left\langle f, \phi \circ W_{G_{-n}}\right\rangle=\lim _{n \rightarrow \infty}\left\langle f, \phi \circ W_{G_{-n}} \circ W_{G_{-(n+2)}}\right\rangle \\
& =\lim _{n \rightarrow \infty}\left\langle f G_{-n}, \phi_{n+2}\right\rangle=\lim _{n \rightarrow \infty} \int_{M^{\mathcal{U}}}\left(f G_{-n}\right)^{\mathcal{U}} d \mu_{n+2} \\
& =\lim _{n \rightarrow \infty} \int_{M^{\mathcal{U}}} f^{\mathcal{U}} G_{-n}^{\mathcal{U}} d \mu_{n+2} \\
& =\lim _{n \rightarrow \infty} \int_{M^{\mathcal{U}}} f^{\mathcal{U}} G_{-n}^{\mathcal{U}} d \mu
\end{aligned}
$$

where the last equality follows from the fact that $G_{-n}^{\mathcal{U}}=0$ on $M^{\mathcal{U}} \backslash A_{n}$ and $\mu_{n+2} \uparrow_{A_{n}}=\mu \uparrow_{A_{n}}$. But $G_{-n}^{\mathcal{U}}$ converge pointwise and increasingly to $\chi_{M^{\mathcal{U}} \backslash\{0\}}$, so by Lebesgue's monotone convergence theorem we obtain

$$
\langle f, \phi\rangle=\lim _{n \rightarrow \infty} \int_{M^{\mathcal{U}}} f^{\mathcal{U}} G_{-n}^{\mathcal{U}} d \mu=\int_{M^{\mathcal{U}}} f^{\mathcal{U}} d \mu .
$$

for every positive $f \in \operatorname{Lip}_{0}(M)$, and hence for every $f \in \operatorname{Lip}_{0}(M)$. 
Since elements of $\mathcal{F}(M)$ also avoid 0 and infinity, appealing to Theorem 4.3.2 we recover one of the implications from [9, Proposition 2.7] as a corollary:

Theorem 4.3.6. Every positive $m \in \mathcal{F}(M)$ can be represented by an almost Radon measure on $M$.

Let us remark that, as is the case with finiteness, full regularity cannot be achieved in Theorems 4.3.5 and 4.3.6 in general. Indeed, it is straightforward to check that any Borel measure $\mu$ representing the functional $m$ constructed in Example 4.3.3 satisfies $\mu(U)=\infty$ for every open neighborhood $U$ of 0 . Therefore, if $\mu$ is outer regular then $\mu(\{0\})=\infty$ and so $\mu$ cannot be $\sigma$-finite.

Although stated in terms of positive elements, Theorems 4.3.6 and 4.3.5 also yield representation results for majorizable elements. For instance, any majorizable $m \in \mathcal{F}(M)$ can be written as the difference between two positive elements and therefore represented as the difference between two almost Radon positive measures. It is tempting to state that $m$ is represented by a signed $\sigma$-finite measure, but that would be inaccurate because of a potential indeterminacy of the form $\infty-\infty$ around the base point. Therefore the most accurate statement would be the following:

Theorem 4.3.7. Let $\phi \in \mathcal{F}(M)^{* *}$. Then the following are equivalent:

(i) $\phi$ is majorizable and avoids 0 and infinity,

(ii) $\phi$ is the difference of two elements of $\mathcal{F}(M)^{* *}$ induced by positive almost Radon measures on $M^{\mathcal{U}}$.

The same holds if $\mathcal{F}(M)^{* *}$ and $M^{\mathcal{U}}$ are replaced by $\mathcal{F}(M)$ and $M$, respectively, and the condition "avoids 0 and infinity" is removed.

Proof. For (i) $\Rightarrow$ (ii), write $\phi=\phi^{+}-\phi^{-}$where $\phi^{ \pm}$are positive and may be assumed to avoid 0 and infinity as in the proof of Theorem 4.3.1, then apply Theorem 4.3.5 (or Theorem 4.3.6 for the $\mathcal{F}(M)$ case). The implication (ii) $\Rightarrow$ (i) follows directly from Lemma 4.2.9.

Let us now summarize our results for a few important particular cases. When $M$ is bounded, every element of $\mathcal{F}(M)^{* *}$ avoids infinity (strongly, in fact) and hence by Theorem 3.5.3 it can be expressed as a derivation at 0 plus a functional that avoids 0 and infinity. So every positive element of $\mathcal{F}(M)^{* *}$ is "a derivation plus a measure":

Corollary 4.3.8. Suppose that $M$ is bounded, and let $\phi \in \mathcal{F}(M)^{* *}$ be positive. Then $\phi=\phi_{0}+\mathcal{L} \mu$ where $\phi_{0}$ is a positive derivation at 0 and $\mu$ is a positive almost Radon measure on $M^{\mathcal{U}}$. If moreover $0 \notin \mathcal{S}(\phi)$, then $\phi_{0}=0$ and $\mu \in \mathcal{M}\left(M^{\mathcal{U}}\right)$. 
If moreover the base point is isolated in $M$ then there are no nonzero derivations at 0 and we get the following characterization, combining with Theorems 4.2.10 and 4.2.12:

Corollary 4.3.9. Suppose that $M$ is bounded and the base point is isolated. Then for $\phi \in \mathcal{F}(M)^{* *}$ the following are equivalent:

(i) $\phi$ is majorizable,

(ii) $\phi$ is represented by a Radon measure on $M^{\mathcal{U}}$,

(iii) $\phi$ is the weak* limit of a net $\left(m_{i}\right)$ in $\operatorname{span} \delta(M)$ such that $\left\|m_{i}\right\|_{1}$ is bounded.

The same holds if we replace $\mathcal{F}(M)^{* *}$ with $\mathcal{F}(M), M^{\mathcal{U}}$ with $M$, "weak* limit" with "norm limit" and "net" with "sequence", respectively.

The most significant particular case of the preceding analysis is given by compact metric spaces $M$. In that case $M^{\mathcal{U}}=M$, so Proposition 4.2 .2 shows that $\mathcal{L} \mu$ actually belongs to $\mathcal{F}(M)$. Thus the majorizable elements of $\mathcal{F}(M)$ and $\mathcal{F}(M)^{* *}$ are almost the same:

Corollary 4.3.10. Suppose that $M$ is compact, and let $\phi \in \mathcal{F}(M)^{* *}$ be majorizable. Then $\phi=m+\phi_{0}$ where $m \in \mathcal{F}(M)$ is majorizable and $\phi_{0}$ is a derivation at 0 . If moreover $0 \notin \mathcal{S}(\phi)$, then $\phi \in \mathcal{F}(M)$ and it is represented by a Radon measure on $M$.

Let us stress that in Corollaries 4.3.8 and 4.3.10 derivations at 0 can only exist if 0 is not an isolated point in $M$ (if the base point is isolated, then it cannot be contained in the extended support of any $\phi \in \mathcal{F}(M)^{* *}$ in view of Proposition 3.5.22). So this provides yet another example where the order structure of Lipschitz-free spaces and their biduals depends on the choice of base point.

\section{Minimal majorants}

Now we turn to the following problem. Given a majorizable functional $\phi$ of $\mathcal{F}(M)$ or $\mathcal{F}(M)^{* *}$, i.e. one that can be expressed as the difference between two positive functionals, does it admit a canonical, minimal representation as such a difference? Let us fix some notation:

Definition 4.3.11. Let $X$ be an ordered vector space, and suppose that $x \in X$ is majorizable. A minimal majorant of $x$ is a majorant $x^{+}$of $x$ with the property that every majorant of $x$ is also a majorant of $x^{+}$.

Note that we are not using the term "minimal" in the usual partial order theoretical sense that there is no strictly smaller majorant of $x$, but rather we require $x^{+}$to be a lower bound for all majorants of $x$. 
Our question can be rephrased as: does every majorizable element have a minimal majorant? It is obvious that the minimal majorant is unique whenever it exists, and that if $x^{+}$is the minimal majorant of $x$ then $x^{-}=x^{+}-x$ is the minimal majorant of $-x$. Moreover, the existence of such a minimal majorant is equivalent to the existence of an optimal representation $x=x^{+}-x^{-}$where $x^{+}, x^{-}$are positive and minimal, i.e. such that for every expression $x=y^{+}-y^{-}$where $y^{+}, y^{-} \geq 0$ we have $y^{+} \geq x^{+}$and $y^{-} \geq x^{-}$. This behavior is found in $\operatorname{Lip}_{0}(M)$ with the optimal decomposition $f=f^{+}-f^{-}$, and also in finite measures where the Jordan decomposition into their positive and negative parts is optimal in that sense. In both cases, the minimal majorant of an element can be identified with its positive part. It is reasonable to expect similar properties from majorizable functionals on $\operatorname{Lip}_{0}(M)$, given their close relationship to measures.

By Theorem 3.5.3, any majorizable functional $\phi \in \mathcal{F}(M)^{* *}$ can be uniquely decomposed as $\phi=\phi_{0}+\phi_{s}+\phi_{\infty}$ where $\phi_{s}$ avoids 0 and infinity, $\phi_{\infty}$ is concentrated at infinity and $\phi_{0}$ is a derivation at 0 , and all of these terms are majorizable. It is straightforward to verify that $\phi$ has a minimal majorant if and only if each term $\phi_{s}, \phi_{\infty}, \phi_{0}$ does. Let us see that this is the case for $\phi_{s}$ :

Theorem 4.3.12. Suppose that $\phi \in \mathcal{F}(M)^{* *}$ avoids 0 and infinity. If $\phi$ is majorizable then it has a minimal majorant that also avoids 0 and infinity. Moreover, $\phi^{+}$and $\phi^{-}=\phi^{+}-\phi$ are represented by positive almost Radon measures on $M^{\mathcal{U}}$ that are concentrated on disjoint Borel subsets of $M^{\mathcal{U}}$.

In the proof we will use the following simple lemma about minimal majorants of weighted functionals:

Lemma 4.3.13. Suppose that $\phi$ is a majorizable element in $\mathcal{F}(M)$ or $\mathcal{F}(M)^{* *}$ that has a minimal majorant $\phi^{+}$. Then, for every $h \in \operatorname{Lip}(M)^{+}$with bounded support, $\phi \circ W_{h}$ is majorizable and its minimal majorant is $\phi^{+} \circ W_{h}$.

Proof. Let $c=\|h\|_{\infty}$, which is finite because $\operatorname{supp}(h)$ is bounded. Notice that $W_{c-h}=c I-W_{h}$ is also a $w^{*}-w^{*}$-continuous operator on $\operatorname{Lip}_{0}(M)$ (where $I$ stands for the identity operator).

It is clear that $\phi \circ W_{h}$ is majorized by $\phi^{+} \circ W_{h} \geq 0$. Now suppose that $\psi \in$ $\left(\mathcal{F}(M)^{* *}\right)^{+}$is another majorant for $\phi \circ W_{h}$, then

$$
\psi+\phi^{+} \circ W_{c-h} \geq \phi \circ W_{h}+\phi \circ W_{c-h}=c \phi .
$$

The minimal majorant of $c \phi$ is obviously $c \phi^{+}$, hence $\psi+\phi^{+} \circ W_{c-h} \geq c \phi^{+}$and therefore $\psi \geq c \phi^{+}-\phi^{+} \circ W_{c-h}=\phi^{+} \circ W_{h}$ as claimed.

Proof of Theorem 4.3.12. We will first prove the theorem under the assumption that $\phi$ avoids 0 and infinity strongly. By Theorem 4.3.1 we have $\phi=\mathcal{L} \mu$ for some 
$\mu \in \mathcal{M}\left(M^{\mathcal{U}}\right)$. Let $\mu=\mu^{+}-\mu^{-}$be the Jordan decomposition of $\mu$. Then $\phi^{+}=\mathcal{L} \mu^{+}$ and $\phi^{-}=\mathcal{L} \mu^{-}$are elements of $\mathcal{F}(M)^{* *}$ by Proposition 4.2.1 (see the remarks after Proposition 4.2.2), and avoid 0 and infinity by Lemma 4.2.9. Clearly $\phi^{+}$is a majorant for $\phi$; we claim that it is minimal. Let $\psi$ be another majorant for $\phi$. By assumption there is $n \in \mathbb{N}$ such that $\phi=\phi \circ W_{\Pi_{n}}$, hence $\psi \geq \psi \circ W_{\Pi_{n}} \geq \phi$. Theorem 4.3.1 implies that $\psi \circ W_{\Pi_{n}}$ is represented by a Radon measure $\lambda \in \mathcal{M}\left(M^{\mathcal{U}}\right)$, which is positive by Proposition 4.2.4. Then $\mathcal{L} \lambda=\psi \circ W_{\Pi_{n}} \geq \mathcal{L} \mu$ and thus $\lambda \geq \mu$ again by Proposition 4.2.4. By the Hahn decomposition theorem, we must have $\lambda \geq \mu^{+}$and therefore $\psi \geq \mathcal{L} \lambda \geq \mathcal{L} \mu^{+}=\phi^{+}$. This proves our claim. Moreover, $\phi=\phi^{+}-\phi^{-}$and $\mu^{+}$and $\mu^{-}$are concentrated on disjoint Borel subsets of $M^{\mathcal{U}}$. That ends the proof of this case.

Let us now handle the general case. For each $n \in \mathbb{N}, \phi \circ W_{\Pi_{n}}$ is majorizable and avoids 0 and infinity strongly, therefore it has a minimal majorant $\phi_{n}^{+}$by the previous paragraph. Now let $\psi \in \mathcal{F}(M)^{* *}$ be any majorant of $\phi$. Then $\psi \geq \psi \circ W_{\Pi_{n}} \geq \phi \circ W_{\Pi_{n}}$ and so $\psi \geq \phi_{n}^{+}$for any $n$. Notice that $\left(\phi_{n}^{+}\right)$is a bounded sequence since

$$
\left\|\phi_{n}^{+}\right\|=\left\langle\rho, \phi_{n}^{+}\right\rangle \leq\langle\rho, \psi\rangle=\|\psi\|,
$$

therefore it must have a $w^{*}$-cluster point $\phi^{+} \in\left(\mathcal{F}(M)^{* *}\right)^{+}$, which will obviously satisfy $\phi^{+} \leq \psi$. Taking weak* limits in $\phi_{n}^{+} \geq \phi \circ W_{\Pi_{n}}$ for the appropriate subnet yields $\phi^{+} \geq \lim _{n} \phi \circ W_{\Pi_{n}}=\phi$. Thus $\phi^{+}$is a majorant for $\phi$, and it is minimal because $\psi$ was arbitrary. This proves existence. Moreover, we have $\phi_{n}^{+}=\phi^{+} \circ W_{\Pi_{n}}$ by Lemma 4.3.13, and taking weak* limits yields $\phi^{+}=\lim _{n} \phi^{+} \circ W_{\Pi_{n}}$, i.e. $\phi^{+}$ avoids 0 and infinity.

Let $\phi^{-}=\phi^{+}-\phi$ and $\phi_{n}^{-}=\phi_{n}^{+}-\phi_{n}$. We have already proved that $\phi_{n}^{+}, \phi_{n}^{-}$are represented by positive Radon measures $\mu_{n}^{+}, \mu_{n}^{-}$concentrated on disjoint Borel sets $A_{n}^{+}, A_{n}^{-}$. Since $\phi_{m}^{+}=\phi^{+} \circ W_{\Pi_{m}} \geq \phi^{+} \circ W_{\Pi_{n}}=\phi_{n}^{+}$for every $m \geq n$ by Lemma 4.3.13, the linearity of $\mathcal{L}$ on Radon measures and Proposition 4.2.4 imply that $\mu_{m}^{+} \geq \mu_{n}^{+}$. Therefore $\mu_{n}^{+}\left(A_{n}^{+} \backslash A_{m}^{+}\right)=0$ and $\mu_{n}^{+}$is concentrated on $A_{m}^{+}$. It follows that each $\mu_{n}^{+}$is concentrated on $\bigcap_{m=n}^{\infty} A_{m}^{+}$, and thus also on the set

$$
A^{+}=\bigcup_{n=1}^{\infty} \bigcap_{m=n}^{\infty} A_{m}^{+} \text {. }
$$

By Theorem 4.3.5, $\phi^{+}$is represented by a positive almost Radon measure $\mu^{+}$on $M^{\mathcal{U}}$. We will show that $\mu^{+}$is the setwise limit of $\mu_{n}^{+}$as $n \rightarrow \infty$. This will imply that $\mu^{+}$is concentrated on $A^{+}$. First note that $\phi_{m}^{+} \leq \phi^{+}$and thus $\mu_{m}^{+} \leq \mu^{+}$by Proposition 4.2.4. Therefore, since $\mu^{+}$is concentrated on $M^{\mathcal{R}} \backslash\{0\}$ by Proposition 4.2 .1 , by inner regularity it will be enough to check that $\mu^{+}(K)=\lim _{m} \mu_{m}^{+}(K)$ for every compact $K \subset M^{\mathcal{R}} \backslash\{0\}$. Fix such a $K$, then there is $n \in \mathbb{N}$ such that $K \subset R_{n}$ where

$$
R_{n}=\left\{\zeta \in M^{\mathcal{U}}: 2^{-n}<\rho^{\mathcal{U}}(\zeta)<2^{n}\right\} .
$$


Let $\varepsilon>0$. By the Radonness of $\mu^{+} \uparrow_{R_{n}}$, we may choose a compact $L \subset R_{n} \backslash K$ with $\mu^{+}\left(R_{n} \backslash(K \cup L)\right) \leq \varepsilon$. Then $K$ and $L \cup\left(M^{\mathcal{U}} \backslash R_{n}\right)$ are compact and disjoint, so let us construct $f \in \operatorname{Lip}_{0}(M)$ such that $0 \leq f \leq 1, f^{\mathcal{U}}=1$ on $K$, and $f^{\mathcal{U}}=0$ on $L \cup\left(M^{\mathcal{U}} \backslash R_{n}\right)$. For every $m \geq n$ we have $f \Pi_{m}=f$, therefore

$$
\begin{aligned}
\mu_{m}^{+}(K) \leq \mu^{+}(K) & \leq\left\langle f, \phi^{+}\right\rangle=\left\langle f \Pi_{m}, \phi^{+}\right\rangle=\left\langle f, \phi_{m}^{+}\right\rangle \\
& \leq \mu_{m}^{+}(K)+\mu_{m}^{+}\left(R_{n} \backslash(K \cup L)\right) \leq \mu_{m}^{+}(K)+\varepsilon .
\end{aligned}
$$

Since $\varepsilon$ was arbitrary, we get $\mu_{m}^{+}(K)=\mu^{+}(K)$ for $m \geq n$ as desired.

Finally, recall that if $\phi^{+}$is the minimal majorant of $\phi$ then $\phi^{-}=\phi^{+}-\phi$ is the minimal majorant of $-\phi$. Therefore a similar argument with $-\phi, \mu_{n}^{-}$and $A_{n}^{-}$shows that the almost Radon measure $\mu^{-}$representing $\phi^{-}$is concentrated on $A^{-}=\bigcup_{n} \bigcap_{m \geq n} A_{m}^{-}$. Since $A^{+}$and $A^{-}$are disjoint Borel subsets of $M^{\mathcal{U}}$, this finishes our proof.

We do not know whether a majorizable functional $\phi$ that is concentrated at infinity must admit a minimal majorant. But if it does, its minimal majorant $\phi^{+}$must also be concentrated at infinity. Indeed, by Lemma 4.3.13, $\phi^{+} \circ W_{H_{n}}$ is the minimal majorant of $\phi \circ W_{H_{n}}=0$, so it must also be equal to 0 for every $n$. The same argument works for minimal majorants of derivations at 0 , replacing $H_{n}$ with $G_{-n}$.

Since majorizable elements of $\mathcal{F}(M)$ avoid 0 and infinity, we can now deduce that they also have minimal majorants. It is possible to prove this following the argument used in Theorem 4.3.12, but we will instead deduce it as a consequence. Moreover, we finally obtain in full generality the promised result that the properties of being majorizable in $\mathcal{F}(M)$ and $\mathcal{F}(M)^{* *}$ are equivalent. We require an additional observation that deserves to be stated independently:

Lemma 4.3.14. Let $\phi, \psi \in \mathcal{F}(M)^{* *}$ be such that $0 \leq \phi \leq \psi$. If $\psi \in \mathcal{F}(M)$, then $\phi \in \mathcal{F}(M)$.

Proof. Let $\left(f_{i}\right)$ be a net of positive functions in $B_{\operatorname{Lip}_{0}(M)}$ that decreases pointwise to 0 . Then we have $0 \leq\left\langle f_{i}, \phi\right\rangle \leq\left\langle f_{i}, \psi\right\rangle$ for each $i$. Since $\psi$ is weak* continuous, $\left\langle f_{i}, \psi\right\rangle \rightarrow 0$ and so $\left\langle f_{i}, \phi\right\rangle \rightarrow 0$ too. Hence $\phi$ is normal, and Theorem 3.2.5 shows that it is $w^{*}$-continuous.

Theorem 4.3.15. Let $m \in \mathcal{F}(M)$. Then $m$ is majorizable in $\mathcal{F}(M)$ if and only if it is majorizable in $\mathcal{F}(M)^{* *}$. In that case, it has a minimal majorant $m^{+}$that belongs to $\mathcal{F}(M)$. Moreover, $m^{+}$and $m^{-}=m^{+}-m$ are represented by positive almost Radon measures on $M$ that are concentrated on disjoint Borel subsets of $M$.

Proof. It is clear that if $m$ is majorizable in $\mathcal{F}(M)$ then it is also majorizable in $\mathcal{F}(M)^{* *}$. Now suppose that $m$ is majorizable in $\mathcal{F}(M)^{* *}$. Since $m$ satisfies the 
hypothesis of Theorem 4.3.12, it has a minimal majorant $m^{+} \in\left(\mathcal{F}(M)^{* *}\right)^{+}$that avoids 0 and infinity. Thus $m^{+}$is the norm limit of $m^{+} \circ W_{\Pi_{n}}$, so in order to prove that $m^{+} \in \mathcal{F}(M)$ it will be enough to show that $m^{+} \circ W_{\Pi_{n}} \in \mathcal{F}(M)$ for every $n \in \mathbb{N}$. Fix $n$, then Lemma 4.3.13 implies that $m^{+} \circ W_{\Pi_{n}}$ is the minimal majorant of $m \circ W_{\Pi_{n}}$. By Theorem 4.3.2 there exists $\mu_{n} \in \mathcal{M}(M)$ such that $m \circ W_{\Pi_{n}}=\mathcal{L} \mu_{n}$. Then $\mathcal{L} \mu_{n}^{+} \in \mathcal{F}(M)^{+}$by the remarks after Proposition 4.2 .2 , and it is clearly a majorant of $m \circ W_{\Pi_{n}}$. Hence by minimality we get that $m^{+} \circ W_{\Pi_{n}} \leq \mathcal{L} \mu_{n}^{+}$, and Lemma 4.3.14 yields that $m^{+} \circ W_{\Pi_{n}} \in \mathcal{F}(M)$ as well. Thus $m$ is majorizable in $\mathcal{F}(M)$, and clearly its minimal majorant in $\mathcal{F}(M)$ is also $m^{+}$.

Finally, by Theorem 4.3.12, $\mathrm{m}^{+}$and $\mathrm{m}^{-}$are represented by positive almost Radon measures $\mu^{+}, \mu^{-}$on $M^{\mathcal{U}}$ concentrated on disjoint Borel sets. But $m^{ \pm} \in \mathcal{F}(M)$, hence $\mu^{+}, \mu^{-}$are actually concentrated on $M$ by Theorem 4.2.7. This ends the proof.

Notice that Theorems 4.3.15 and 4.3.12 show that majorizable elements in $\mathcal{F}(M)$ and $\mathcal{F}(M)^{* *}$ (that avoid 0 and infinity) can almost be represented as measures with a Hahn decomposition: in general they cannot be represented as a single measure, but they are always given by a difference of two minimal positive measures that are concentrated on disjoint Borel sets. Let us remark that this separation property is valid only in terms of the Borel sets on which the measures concentrate but not for their closed supports, hence neither for the supports of the functionals themselves, i.e. we can find simple examples where $\operatorname{supp}\left(m^{+}\right) \cap \operatorname{supp}\left(m^{-}\right) \neq \varnothing$.

We conclude this section by introducing a notion of variation for majorizable functionals in analogy with measures. The existence of the variation for majorizable functionals avoiding 0 and infinity, in particular for elements of $\mathcal{F}(M)$, is guaranteed by Theorem 4.3.12.

Definition 4.3.16. Suppose that $\phi \in \mathcal{F}(M)^{* *}$ is majorizable. If $\phi=\phi^{+}-\phi^{-}$ where $\phi^{+}$is the minimal majorant of $\phi$, we call the variation of $\phi$ the functional $|\phi| \in \mathcal{F}(M)^{* *}$ defined by

$$
|\phi|=\phi^{+}+\phi^{-} .
$$

Note that by Theorem 4.3.15 the variation of a majorizable element from $\mathcal{F}(M)$ also belongs to $\mathcal{F}(M)$. The variation of $\phi$ is obviously also its majorant, but moreover, it majorizes the modulus of $\phi$ in the following sense:

Proposition 4.3.17. Let $\phi \in \mathcal{F}(M)^{* *}$ avoid 0 and infinity and let it be majorizable. Then the variation of $\phi$ satisfies

$$
|\langle f, \phi\rangle| \leq\langle|f|,|\phi|\rangle
$$

for every $f \in \operatorname{Lip}_{0}(M)$. Moreover, $|\phi|$ is the smallest element of $\mathcal{F}(M)^{* *}$ with this property. 
Proof. Assume first that $f \in \operatorname{Lip}_{0}(M)$ and $f \geq 0$. Then

$$
\langle f, \phi\rangle \leq\left\langle f, \phi^{+}\right\rangle \leq\langle f,|\phi|\rangle
$$

and

$$
-\langle f, \phi\rangle=\langle f,-\phi\rangle \leq\left\langle f, \phi^{-}\right\rangle \leq\langle f,|\phi|\rangle,
$$

hence $|\langle f, \phi\rangle| \leq\langle f,|\phi|\rangle$. Now decompose any $f \in \operatorname{Lip}_{0}(M)$ as $f=f^{+}-f^{-}$where $f^{+}, f^{-} \geq 0$. Then

$$
|\langle f, \phi\rangle| \leq\left|\left\langle f^{+}, \phi\right\rangle\right|+\left|\left\langle f^{-}, \phi\right\rangle\right| \leq\left\langle f^{+},|\phi|\right\rangle+\left\langle f^{-},|\phi|\right\rangle=\langle|f|,|\phi|\rangle .
$$

Now suppose that $\psi \in \mathcal{F}(M)^{* *}$ is such that $|\langle f, \phi\rangle| \leq\langle|f|, \psi\rangle$ for every $f \in$ $\operatorname{Lip}_{0}(M)$, which clearly implies $\psi \geq 0$. Then $\psi_{s}=\lim _{n} \psi \circ W_{\Pi_{n}}$ has the same property, so we may assume that $\psi$ avoids 0 and infinity. For positive $f$ we get $\langle f, \psi\rangle \geq|\langle f, \phi\rangle|$, hence $\psi \geq \phi$ and $\psi \geq-\phi$. Thus, using the minimal majorants, we must also have $\psi \geq \phi^{+}$and $\psi \geq(-\phi)^{+}=\phi^{-}$. By Theorem 4.3.5, $\phi^{+}, \phi^{-}$and $\psi$ are represented by positive almost Radon measures $\mu^{+}, \mu^{-}$and $\lambda$ on $M^{\mathcal{U}}$, so Proposition 4.2.4 yields $\lambda \geq \mu^{+}$and $\lambda \geq \mu^{-}$. However, by Theorem $4.3 .12 \mu^{+}$and $\mu^{-}$are concentrated on disjoint Borel sets, and so it is immediate that we actually have $\lambda \geq \mu^{+}+\mu^{-}$. Thus $\psi \geq \phi^{+}+\phi^{-}=|\phi|$ by Proposition 4.2.4.

We finish by establishing the intuitively obvious fact that passing from a majorizable functional to its minimal majorant or its variation does not increase its support.

Proposition 4.3.18. Let $\phi \in \mathcal{F}(M)^{* *}$ be majorizable and avoid 0 and infinity. Then

$$
\mathcal{S}(|\phi|)=\mathcal{S}\left(\phi^{+}\right) \cup \mathcal{S}\left(\phi^{-}\right)=\mathcal{S}(\phi) .
$$

If $\phi \in \mathcal{F}(M)$, the equality holds also for supports in $\mathcal{F}(M)$ in place of extended supports.

Proof. Apply first Corollary 3.5.9 to observe that $\mathcal{S}(|\phi|) \subset \mathcal{S}\left(\phi^{+}\right) \cup \mathcal{S}\left(\phi^{-}\right)$and $\mathcal{S}(\phi) \subset \mathcal{S}\left(\phi^{+}\right) \cup \mathcal{S}\left(\phi^{-}\right)$. The inclusion $\mathcal{S}\left(\phi^{+}\right) \cup \mathcal{S}\left(\phi^{-}\right) \subset \mathcal{S}(|\phi|)$ follows from Corollary 3.5.10(c). Thus it only remains to be proved that $\mathcal{S}\left(\phi^{+}\right), \mathcal{S}\left(\phi^{-}\right) \subset$ $\mathcal{S}(\phi)$. It will suffice to show that $\mathcal{S}\left(\phi^{+}\right) \subset \mathcal{S}(\phi)$, then $\mathcal{S}\left(\phi^{-}\right) \subset \mathcal{S}(\phi)$ follows from Corollary 3.5.9 again. Moreover, $\phi^{+}$avoids infinity by Theorem 4.3.12, hence in view of Proposition 3.5.21 it is enough to prove that $\mathcal{S}\left(\phi^{+}\right) \cap M^{\mathcal{R}} \subset \mathcal{S}(\phi)$.

Let $\zeta \in M^{\mathcal{R}} \backslash \mathcal{S}(\phi)$. By Proposition 3.5.6 there is a neighbourhood $U$ of $\zeta$ such that $\langle f, \phi\rangle=0$ for any $f \in \operatorname{Lip}_{0}(M)$ with the support contained in $U \cap M$. We may moreover assume that $U \subset\left\{\xi \in M^{\mathcal{U}}: \rho^{\mathcal{U}}(\xi)<\rho^{\mathcal{U}}(\zeta)+1\right\}$, so that $U \cap M$ is bounded. Take other neighbourhoods $V, W$ of $\zeta$ such that $\bar{W}^{\mathcal{U}} \subset V \subset \bar{V}^{\mathcal{U}} \subset U$ and define $h \in \operatorname{Lip}(M)$ so that $0 \leq h \leq 1$ and that $h=1$ on $W \cap M$ and $h=0$ 
on $M \backslash V$. The support of such $h$ is bounded, so we may define $\psi=\phi^{+} \circ W_{1-h}$. Then $\psi \leq \phi^{+}$, and for any positive $f \in \operatorname{Lip}_{0}(M)$ we have

$$
\langle f, \psi\rangle=\left\langle f(1-h), \phi^{+}\right\rangle \geq\langle f(1-h), \phi\rangle=\langle f, \phi\rangle-\langle f h, \phi\rangle=\langle f, \phi\rangle
$$

since $\operatorname{supp}(f h) \subset U \cap M$. Thus $\psi$ is a majorant for $\phi$, and from the minimality of $\phi^{+}$it follows that $\phi^{+}=\psi$. Hence $\phi^{+} \circ W_{h}=0$. But then $\left\langle f, \phi^{+}\right\rangle=\left\langle f h, \phi^{+}\right\rangle=0$ for any $f \in \operatorname{Lip}_{0}(M)$ such that $\operatorname{supp}(f) \subset W \cap M$. Therefore $\zeta \notin \mathcal{S}\left(\phi^{+}\right)$by Proposition 3.5.6. This completes the proof for functionals in $\operatorname{Lip}_{0}(M)^{*}$.

If $\phi \in \mathcal{F}(M)$, then also $\phi^{+}, \phi^{-} \in \mathcal{F}(M)$ by Theorem 4.3.15, and the equality for supports in $\mathcal{F}(M)$ follows by intersecting with $M$ and applying Corollary 3.5.7.

\subsection{Radially discrete spaces}

We have already witnessed that, in general, not all elements of a Lipschitz-free space $\mathcal{F}(M)$ can be represented by a measure, or as the difference between two positive elements. In this section, we will identify the scenarios where such representations are always possible. To this end, we introduce two classes of metric spaces:

Definition 4.4.1. We will say that a pointed metric space $M$ is radially discrete if there exists $\alpha>0$ such that $d(x, y) \geq \alpha \cdot d(x, 0)$ for every pair $x, y$ of distinct points of $M$. We will say that $M$ is radially uniformly discrete if it is radially discrete and uniformly discrete.

Note that if $M$ is radially discrete, then the set $M \backslash B(0, r)$ is uniformly discrete for every $r>0$, and its uniform separation constant increases linearly with $r$. In particular, every point of $M \backslash\{0\}$ is isolated. However the base point need not be isolated, so in particular $M$ is not necessarily discrete. In fact, $M$ is uniformly discrete if and only if the base point is also isolated. Thus, the property of being radially discrete depends on the choice of base point, but it is easy to see that the property of being radially uniformly discrete does not.

Notice also that if $M$ is uniformly discrete and bounded then it is also radially uniformly discrete, taking $\alpha=\theta(M) / \operatorname{diam}(M)$ where $\theta(M)$ is the uniform separation constant of $M$. Unbounded uniformly discrete spaces are not necessarily radially discrete: consider e.g. $M=\mathbb{N}$ with the metric inherited from $\mathbb{R}$.

The following result is our main reason to introduce this class of metric spaces:

Theorem 4.4.2. $M$ is radially discrete if and only if all elements of $\mathcal{F}(M)$ are majorizable. 
Proof. Suppose first that $M$ is radially discrete, and let $\alpha>0$ be such that $d(x, y) \geq \alpha \cdot d(x, 0)$ whenever $x \neq y \in M$. Now let $m \in \mathcal{F}(M)$, and use Proposition 2.3.2 to write $m$ as a series of molecules

$$
m=\sum_{n=1}^{\infty} a_{n} m_{x_{n} y_{n}}=\sum_{n=1}^{\infty} a_{n} \frac{\delta\left(x_{n}\right)-\delta\left(y_{n}\right)}{d\left(x_{n}, y_{n}\right)}
$$

where $x_{n} \neq y_{n} \in M$ and $\sum_{n}\left|a_{n}\right|<\infty$. We may assume that $a_{n} \geq 0$ without loss of generality. Now notice that

$$
\left\|\sum_{n=1}^{N} \frac{a_{n}}{d\left(x_{n}, y_{n}\right)} \delta\left(x_{n}\right)\right\| \leq \sum_{n=1}^{N} a_{n} \frac{d\left(x_{n}, 0\right)}{d\left(x_{n}, y_{n}\right)} \leq \frac{1}{\alpha} \sum_{n=1}^{N} a_{n}
$$

for every $N \in \mathbb{N}$, so the series

$$
m^{\prime}=\sum_{n=1}^{\infty} \frac{a_{n}}{d\left(x_{n}, y_{n}\right)} \delta\left(x_{n}\right)
$$

is absolutely convergent. Then $m^{\prime}$ is a positive element of $\mathcal{F}(M)$, and it is clear that $\langle m, f\rangle \leq\left\langle m^{\prime}, f\right\rangle$ for any $f \geq 0$ in $\operatorname{Lip}_{0}(M)$. Hence $m \leq m^{\prime}$ and $m$ is majorizable.

For the converse implication, assume that every element of $\mathcal{F}(M)$ is majorizable. We will first show that every point of $M$ is isolated, except possibly the base point. Indeed, let $x \in M \backslash\{0\}$ and suppose that it is not an isolated point of $M$. Then we can find sequences $\left(x_{n}\right)$ and $\left(y_{n}\right)$ of points of $M \backslash\{0\}$, all of them different from each other, that converge to $x$ and such that

$$
0<d\left(x_{n+1}, y_{n+1}\right)<\frac{1}{2} d\left(x_{n}, y_{n}\right)
$$

for every $n \in \mathbb{N}$. For $n \in \mathbb{N}$, let $f_{n} \in \operatorname{Lip}_{0}(M)$ be such that $0 \leq f_{n} \leq 1$, $f_{n}\left(x_{k}\right)=1$ for $k \leq n, f_{n}\left(x_{k}\right)=0$ for $k>n$, and $f_{n}\left(y_{k}\right)=0$ for all $k \in \mathbb{N}$. Also let $h \in \operatorname{Lip}_{0}(M)$ be such that $h\left(x_{k}\right)=h\left(y_{k}\right)=1$ for every $k \in \mathbb{N}$. Now let $m=\sum_{n=1}^{\infty}\left(\delta\left(x_{n}\right)-\delta\left(y_{n}\right)\right)$ and notice that $m \in \mathcal{F}(M)$ as the series is absolutely convergent. By hypothesis there is a positive $m^{+} \in \mathcal{F}(M)$ with $m^{+} \geq m$, and we may assume that $\operatorname{supp}\left(m^{+}\right) \subset \operatorname{supp}(m)$ by Proposition 4.3.18. Thus $f_{n} \leq h$ on $\operatorname{supp}\left(m^{+}\right)$, and we get

$$
\left\langle m^{+}, h\right\rangle \geq\left\langle m^{+}, f_{n}\right\rangle \geq\left\langle m, f_{n}\right\rangle=n
$$

for every $n$. This is a contradiction. So $x$ must be isolated, as we claimed.

Suppose now, for contradiction, that $M$ is not radially discrete. We claim that we may find sequences $\left(x_{n}\right)$ and $\left(y_{n}\right)$ of points of $M \backslash\{0\}$, all of them different from each other, such that $d\left(x_{n}, y_{n}\right) \leq 2^{-n} d\left(x_{n}, 0\right)$. Indeed, we proceed by induction. Choose any pair $\left(x_{1}, y_{1}\right)$ such that $d\left(x_{1}, y_{1}\right)<\frac{1}{2} d\left(x_{1}, 0\right)$. Now suppose that the 
different points $x_{1}, y_{1}, \ldots, x_{n-1}, y_{n-1}$ have been selected. Since all of them are isolated, we may choose $\delta>0$ such that none of them have any point of $M$ at distance less than or equal to $\delta$. We may also take $R>0$ such that all of them are contained in $B(0, R)$. Now let

$$
\alpha=\min \left\{2^{-n}, \frac{\delta}{\delta+R}\right\}
$$

and, using the fact that $M$ is not radially discrete, choose a pair $\left(x_{n}, y_{n}\right)$ of different points such that $d\left(x_{n}, y_{n}\right)<\alpha \cdot d\left(x_{n}, 0\right)$. It is easy to check that neither of them can be 0 . If $y_{n}$ was one of the points $x_{k}$ or $y_{k}$ with $k<n$ then we would have

$$
d\left(x_{n}, y_{n}\right) \leq \frac{\alpha}{1-\alpha} d\left(y_{n}, 0\right) \leq \frac{\delta}{R} d\left(y_{n}, 0\right) \leq \delta
$$

so it would have a point $x_{n}$ at distance less than or equal to $\delta$, which is impossible by construction. Analogously we check that $x_{n}$ cannot be one of the points $x_{k}, y_{k}$ with $k<n$. Thus the points $x_{1}, y_{1}, \ldots, x_{n}, y_{n}$ are all different, and the claim is proved.

Let

$$
m=\sum_{n=1}^{\infty} \frac{\delta\left(x_{n}\right)-\delta\left(y_{n}\right)}{d\left(x_{n}, 0\right)}=\sum_{n=1}^{\infty} \frac{d\left(x_{n}, y_{n}\right)}{d\left(x_{n}, 0\right)} m_{x_{n} y_{n}}
$$

and notice that the norm of the $n$-th term is bounded by $2^{-n}$, so the series is absolutely convergent. For any $n \in \mathbb{N}$, let $f_{n} \in \operatorname{Lip}_{0}(M)$ be such that $f_{n} \geq 0$, $f_{n}\left(x_{k}\right)=d\left(x_{k}, 0\right)$ for $k \leq n, f_{n}\left(x_{k}\right)=0$ for $k>n$, and $f_{n}\left(y_{k}\right)=0$ for all $k \in \mathbb{N}$. Once again, by hypothesis and using Proposition 4.3 .18 we find a positive $m^{+} \in \mathcal{F}(M)$ such that $m^{+} \geq m$ and $\operatorname{supp}\left(m^{+}\right) \subset \operatorname{supp}(m)$. But then $f_{n} \leq \rho$ on $\operatorname{supp}\left(m^{+}\right)$and

$$
\left\langle m^{+}, \rho\right\rangle \geq\left\langle m^{+}, f_{n}\right\rangle \geq\left\langle m, f_{n}\right\rangle=n
$$

for every $n$, a contradiction. This finishes the proof.

Combining this with our previous results on the relation between majorizability and integral representation, we obtain a characterization of those metric spaces such that every element of $\mathcal{F}(M)$ is represented by a Radon measure on $M$. Note that this extends [53, Theorem 3.19] which covers just the compact case.

Corollary 4.4.3. The following are equivalent:

(i) $M$ is radially uniformly discrete,

(ii) all elements of $\mathcal{F}(M)$ are represented by Radon measures on $M$,

(iii) all elements of $\mathcal{F}(M)$ are majorizable, and the base point of $M$ is isolated. 
Proof. (i) $\Leftrightarrow($ iii): This follows immediately from Theorem 4.4.2 and Definition 4.4.1.

(iii) $\Rightarrow$ (ii): If 0 is isolated in $M$ then it is not contained in the support of any element of $\mathcal{F}(M)$, so this implication follows immediately from Theorem 4.3.2.

(ii) $\Rightarrow$ (iii): It follows from Proposition 4.3.4 that the base point of $M$ is isolated. Hence it cannot belong to the support of any element of $\mathcal{F}(M)$, and Theorem 4.3.2 yields the desired conclusion.

\subsection{Open problems}

Theorems 4.2.12 and 4.2.10 characterize the functionals in $\mathcal{F}(M)\left(\operatorname{resp} . \mathcal{F}(M)^{* *}\right)$ that can be represented by a Radon measure on $M$ (resp. $M^{\mathcal{U}}$ ) as those that can be obtained as limits of finitely supported functionals with a bounded sum of coefficients. However these characterizations are limited to functionals that avoid infinity strongly (unless positivity is also assumed). They are valid in general if $M$ is bounded, but Examples 4.2.11 and 4.2.14 show that they can fail in the unbounded case. So we may ask for a full characterization that is valid in all cases:

Problem 4.1. Find a characterization of elements of $\mathcal{F}(M)\left(\right.$ resp. $\left.\mathcal{F}(M)^{* *}\right)$ that can be represented by a Radon measure on $M$ (resp. $M^{\mathcal{U}}$ ) that is valid also when $M$ is unbounded.

The following issue is related to Proposition 4.2.2, showing that if a regular Borel measure on $M$ induces a functional in $\mathcal{F}(M)^{* *}$ then this functional is weak* continuous. If we do not assume regularity of the measure, then we get the same conclusion if $M$ is separable, but our proof fails for nonseparable $M$. Therefore we ask:

Problem 4.2. Does every Borel measure $\mu$ on $M$ such that $\int_{M} \rho d|\mu|<\infty$ induce a weak* continuous functional $\mathcal{L} \mu \in \mathcal{F}(M)$ ?

Note that a negative answer to this question would also solve Problem 3.2, as the corresponding $\mathcal{L} \mu$ would be sequentially normal (by dominated convergence) but not normal. This question may also be related to Problem 3.3, asking whether extended supports are always separable. There is a chance that Problem 4.2 is undecidable and its solution involves metric spaces whose density character is a measurable cardinal.

The next question is probably the most important one in this section and concerns the existence of minimal majorants. 
Problem 4.3. Do all majorizable functionals in $\mathcal{F}(M)^{* *}$ admit a minimal majorant?

We have proved in Theorem 4.3.12 that the answer is positive for functionals that avoid 0 and infinity. By the decomposition from Theorem 3.5.3, it is enough to consider two remaining cases: functionals that are concentrated at infinity, and derivations at the base point.

Our last question is unlikely to have any practical application, but it is still an interesting mathematical curiosity. Let us notice that all higher duals $\mathcal{F}(M)^{* * *}$, $\mathcal{F}(M)^{* * * *}, \ldots$ can be inductively endowed with a vector space order. We have seen in Corollary 4.3.10 that all positive (and hence also majorizable) elements of $\mathcal{F}(M)^{* *}$ are actually elements of $\mathcal{F}(M)$ when $M$ is compact and 0 is an isolated point. So we may ask the following:

Problem 4.4. If $M$ is compact and the base point is isolated, does it follow that every positive element of the successive duals $\mathcal{F}(M)^{(2 n)}$ of even order belongs to $\mathcal{F}(M) ?$ 


\section{Chapter 5}

\section{Extremal structure of the unit ball}

In this last chapter we will use the theory of supports in $\mathcal{F}(M)$ developed in Chapter 3 to analyze the extremal structure of the unit ball of $\mathcal{F}(M)$. More specifically, we will give complete characterizations of all of its preserved extreme points and of its extreme and exposed points that have finite support. We will also completely describe extreme, exposed and preserved extreme points of the positive unit ball. These results appeared originally on a series of papers obtained in collaboration with Guirao, Pernecká, Petitjean and Procházka $[1,5,6]$. Here we have slightly modified the original proofs in an attempt to give a unified treatment to all of these results.

\subsection{Extreme points and metric alignment}

Let us begin by recalling the definition of the most usual types of extremal elements in Banach spaces. There are more notions than those listed here but they fall outside of the scope of this dissertation; one possible reference is [32].

Definition 5.1.1. Let $C$ be a convex subset of a Banach space $X$ and $x \in C$. Then $x$ is

- an extreme point of $C$ if it cannot be written as $x=\frac{1}{2}(y+z)$ with $y, z \in$ $C \backslash\{x\}$,

- an exposed point of $C$ if there is $x^{*} \in X^{*}$ such that $\left.\left\langle x, x^{*}\right\rangle\right\rangle\left\langle y, x^{*}\right\rangle$ for any $y \in C \backslash\{x\}$,

- a preserved extreme point of $C$ if it is an extreme point of $\bar{C}^{w^{*}}$ in $X^{* *}$, 
- a denting point of $C$ if there are slices of $C$ (that is, sets of the form $\left\{y \in C:\left\langle y, x^{*}\right\rangle>\alpha\right\}$ for some $x^{*} \in X^{*}$ and $\left.\alpha \in \mathbb{R}\right)$ of arbitrarily small diameter containing $x$,

- a strongly exposed point of $C$ if there is a fixed $x^{*} \in X^{*}$ such that there are slices of $C$ of the form $\left\{y \in C:\left\langle y, x^{*}\right\rangle>\alpha\right\}$ of arbitrarily small diameter containing $x$.

The set of extreme points of $C$ will be denoted by $\operatorname{ext}(C)$. Our efforts will be focused on the case $C=B_{\mathcal{F}(M)}$, but later we shall also consider the positive unit ball $C=B_{\mathcal{F}(M)}^{+}$.

Among the notions presented above, that of extreme point is the weakest one. In fact, we have the chain of implications described in the following diagram. In the general setting of Banach spaces there are counterexamples for the reverse of each implication, but we shall see that the situation is different when we restrict our attention to the unit balls of Lipschitz-free spaces.

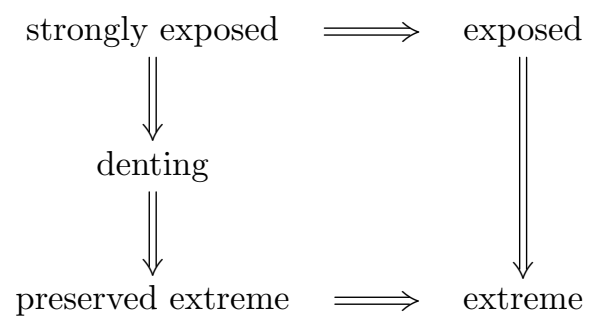

With this setup in place, we may start working on the problem of characterizing all of these types of extremal elements in the unit ball of a given Lipschitz-free space. Historically, the first serious breakthrough in this research program was achieved by Weaver in 1995 [51], when he proved the following:

Theorem 5.1.2 ([53, Corollary 3.44]). Any preserved extreme point of $B_{\mathcal{F}(M)}$ is an elementary molecule in $M$.

This result cements the status of elementary molecules as the most important elements of $\mathcal{F}(M)$, but more importantly, it opens the way for simple geometric characterizations of extremal elements of $B_{\mathcal{F}(M)}$. Indeed, since a molecule $m_{p q}$ is determined by just two points $p, q \in M$, it should be possible to express any such characterization in terms of an equivalent condition on the pair $p, q$ that only involves the metric structure of $M$.

After Weaver's early result, progress in this program was mostly stalled for about 20 years. The first new results to appear were the following, due to García-Lirola, Petitjean, Procházka and Rueda Zoca: 
Theorem 5.1.3 ([24, Theorem 5.4]). Let $p \neq q \in M$. Then $m_{p q}$ is a strongly exposed point of $B_{\mathcal{F}(M)}$ if and only if there is $C>0$ such that

$$
\min \{d(x, p), d(x, q)\} \leq C \cdot(d(p, x)+d(q, x)-d(p, q))
$$

for all $x \in M$.

Theorem 5.1.4 ([23, Theorem 2.4]). Every preserved extreme point of $B_{\mathcal{F}(M)}$ is also a denting point of $B_{\mathcal{F}(M)}$.

Shortly afterwards the author of this dissertation, in collaboration with A. Guirao [1] and E. Pernecká [5], gave similar geometric characterizations of those molecules $m_{p q}$ that are extreme or preserved extreme points of $B_{\mathcal{F}(M)}$ similar to that described in Theorem 5.1.3. These results (Theorems 5.2.6 and 5.2.9) will be proved in the next section. It should be remarked here that it is not known whether all extreme points must be molecules (this is only known for preserved extreme points), so this will not provide a full characterization of all extreme points of $B_{\mathcal{F}(M)}$ yet.

It turns out that these characterizations are very strongly related to notions of metric alignment of points in the metric space $M$, so we will first define these concepts and describe their links to extremality.

Definition 5.1.5. Let $p, q \in M$. The metric segment $[p, q]$ is defined as the set

$$
[p, q]=\{x \in M: d(p, q)=d(p, x)+d(q, x)\} .
$$

We say that three points $p, q, r \in M$ are metrically aligned if one of them belongs to the metric segment spanned by the other two.

Observe that these definitions of metric segment and metric alignment agree with the corresponding intuitive notions of alignment in the Euclidean plane or space. More generally, if $M$ is a strictly convex normed space, then the segment $[p, q]$ coincides with the usual linear segment $\{\lambda p+(1-\lambda) q: \lambda \in[0,1]\}$, and $p, q, r$ are metrically aligned if and only if they are linearly aligned, i.e. if they span an affine subspace of dimension 1 instead of 2, or equivalently, if $p-r$ and $q-r$ are linearly dependent. For a general metric space $M$ the segment $[p, q]$ is closed, always contains $p$ and $q$, and it is possible for it to contain no other point.

The following is a quantitative way of measuring how far three given points are from being metrically aligned:

Definition 5.1.6. Let $p, q, x \in M$. The Gromov product of $p$ and $q$ at $x$ is defined as the value

$$
(p, q)_{x}=\frac{1}{2}(d(p, x)+d(q, x)-d(p, q)) .
$$


Note that $(p, q)_{x} \geq 0$ by the triangle inequality, and $(p, q)_{x}=0$ if and only if $x \in$ $[p, q]$. Note also that $(p, q)_{x} \leq d(x,[p, q])$ for any $x \in M$; this is proved by adding the triangle inequalities $d(p, x) \leq d(p, r)+d(r, x)$ and $d(q, x) \leq d(q, r)+d(r, x)$ for $r \in[p, q]$. Finally, it is straightforward to check that the identity

$$
(p, q)_{x}+(x, q)_{y}=(p, q)_{y}+(p, y)_{x}
$$

holds for any $p, q, x, y \in M$; we will need this later.

Although this may not be apparent at first sight, we will also require partial extensions of these concepts to the uniform compactification $M^{\mathcal{U}}{ }^{1}$ The mapping $x \mapsto(p, q)_{x}$ is clearly 1-Lipschitz, so it is possible to extend it to a mapping $M^{\mathcal{U}} \rightarrow[0, \infty]$. We will denote by

$$
(p, q)_{\zeta}=\lim _{i}(p, q)_{x_{i}}
$$

the evaluation of the corresponding extension at $\zeta \in M^{\mathcal{U}}$ (where $\left(x_{i}\right)$ is a net in $M$ that converges to $\zeta$ ), without explicitly writing the superscript $\mathcal{U}$. We can then consider the following subset of $M^{\mathcal{U}}$ :

Definition 5.1.7. Let $p, q \in M$. The extended metric segment $[p, q]_{\mathcal{U}}$ is defined as the set

$$
[p, q]_{\mathcal{U}}=\left\{\zeta \in M^{\mathcal{U}}:(p, q)_{\zeta}=0\right\}
$$

It should be obvious that $[p, q]_{\mathcal{U}}$ is always compact, contains $p$ and $q$, and that $[p, q]=[p, q]_{\mathcal{U}} \cap M$. We will say that the segment $[p, q]$, resp. the extended segment $[p, q]_{\mathcal{U}}$, is trivial if it only contains the points $p$ and $q$.

The following lemma describes the basic link between metric alignment in $M$ and the extremal structure of $B_{\mathcal{F}(M)}$. Indeed, there is a direct relation between trivial segments and molecules $m_{p q}$ that are extremal elements of the unit ball.

Lemma 5.1.8. Let $p, q$ be distinct points of $M$.

(a) If $m_{p q}$ is an extreme point of $B_{\mathcal{F}(M)}$, then $[p, q]$ is trivial.

(b) If $m_{p q}$ is a preserved extreme point of $B_{\mathcal{F}(M)}$, then $[p, q]_{\mathcal{U}}$ is trivial.

Proof. (a) For any $x \in M \backslash\{p, q\}$ we have

$$
m_{p q}=\frac{\delta(p)-\delta(q)}{d(p, q)}=\frac{\delta(p)-\delta(x)}{d(p, q)}+\frac{\delta(x)-\delta(q)}{d(p, q)}=\frac{d(p, x)}{d(p, q)} m_{p x}+\frac{d(x, q)}{d(p, q)} m_{x q} .
$$

If $x \in[p, q]$ i.e. $d(p, q)=d(p, x)+d(q, x)$, then this expresses $m_{p q}$ as a convex combination of elements $m_{p x}$ and $m_{x q}$ of $B_{\mathcal{F}(M)}$ so it cannot be an extreme point.

\footnotetext{
${ }^{1}$ It is also possible to work with the Stone-Čech compactification $\beta M$ instead, as was done in the original treatment of this subject in $[1,5]$.
} 
(b) Suppose that $(p, q)_{\xi}=0$ for some $\xi \in M^{\mathcal{U}} \backslash\{p, q\}$. Define the functions $\rho_{p}(x)=d(x, p)$ and $\rho_{q}(x)=d(x, q)$ for $x \in M$. It is clear then that

$$
\rho_{p}^{\mathcal{U}}(\xi)+\rho_{q}^{\mathcal{U}}(\xi)-d(p, q)=(p, q)_{\xi}=0
$$

so we may find a bounded net $\left(x_{i}\right)$ in $M$ that converges to $\xi$, hence $\xi \in M^{\mathcal{R}}$ and $\delta(\xi) \in \mathcal{F}(M)^{* *}$. Now define the generalized molecules

$$
m_{p \xi}=\frac{\delta(p)-\delta(\xi)}{\rho_{p}^{\mathcal{U}}(\xi)} \quad \text { and } \quad m_{\xi q}=\frac{\delta(\xi)-\delta(q)}{\rho_{q}^{\mathcal{U}}(\xi)}
$$

Notice that $m_{p \xi}$ is a well-defined element of $\mathcal{F}(M)^{* *}$ since $\rho_{p}^{\mathcal{U}}(\xi)>0$ as $\xi \neq p$. Moreover, for any $f \in B_{\operatorname{Lip}_{0}(M)}$ we have

$$
|\langle f, \delta(p)-\delta(\xi)\rangle|=\left|f(p)-f^{\mathcal{U}}(\xi)\right|=\lim _{i}\left|f(p)-f\left(x_{i}\right)\right| \leq \lim _{i} d\left(p, x_{i}\right)=\rho_{p}^{\mathcal{U}}(\xi)
$$

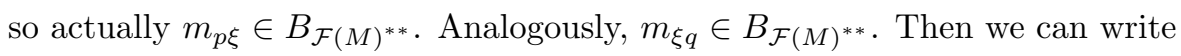

$$
m_{p q}=\frac{\delta(p)-\delta(q)}{d(p, q)}=\frac{\delta(p)-\delta(\xi)}{d(p, q)}+\frac{\delta(\xi)-\delta(q)}{d(p, q)}=\frac{\rho_{p}^{\mathcal{U}}(\xi)}{d(p, q)} m_{p \xi}+\frac{\rho_{q}^{\mathcal{U}}(\xi)}{d(p, q)} m_{\xi q}
$$

i.e. $m_{p q}$ is a convex combination of elements in $B_{\mathcal{F}(M)^{* *}}$, so it cannot be a preserved extreme point.

Lemma 5.1.8, part (a) of which is folklore, shows that trivial (extended) segments are a necessary condition for (preserved) extremality of molecules. In fact they are also sufficient conditions, but the proof of this fact is much more elaborate. The next section will be devoted to that.

The condition that $[p, q]=\{p, q\}$ has a rather clear geometric interpretation. It will be convenient to find a similar equivalent condition to $[p, q]_{\mathcal{U}}=\{p, q\}$ that is stated purely in terms of the geometry of $M$. That condition essentially means that it is not possible to have $d\left(p, x_{i}\right)+d\left(q, x_{i}\right) \rightarrow d(p, q)$ unless $\left(x_{i}\right)$ clusters at $p$ or $q$. Equivalently, the triangle inequality is uniformly strict for $x$ away from $p, q$. The precise formulation is the following:

Lemma 5.1.9. Let $p, q$ be distinct points of $M$. Then the following are equivalent:

(i) $[p, q]_{\mathcal{U}}$ is trivial,

(ii) for every $\varepsilon>0$ there is $\delta>0$ such that $(p, q)_{x} \geq \delta$ whenever $x \in M$ satisfies $\min \{d(p, x), d(q, x)\} \geq \varepsilon$.

Proof. Suppose (i) is false and there is $\xi \in M^{\mathcal{U}} \backslash\{p, q\}$ such that $(p, q)_{\xi}=0$. Then there is a net $\left(x_{i}\right)$ in $M$ such that $x_{i} \rightarrow \xi$ and $(p, q)_{x_{i}} \rightarrow 0$. Choose $\varepsilon>0$ such 
that $d\left(x_{i}, p\right)>\varepsilon$ and $d\left(x_{i}, q\right)>\varepsilon$ eventually; such an $\varepsilon$ exists because $\left\{x_{i}\right\}$ would otherwise have a subsequence that converges to $p$ or $q$. Then (ii) is false for this $\varepsilon$.

Suppose now that (ii) is false, and choose $\varepsilon>0$ such that for every $n \in \mathbb{N}$ there is $x_{n} \in X$ such that $d\left(p, x_{n}\right) \geq \varepsilon, d\left(q, x_{n}\right) \geq \varepsilon$ and $(p, q)_{x_{n}}<2^{-n}$. Let $\xi$ be a cluster point of $\left(x_{n}\right)$ in $M^{\mathcal{U}}$. Then clearly $(p, q)_{\xi}=0$ and $\xi \neq p, q$, so (i) is false.

\subsection{Extreme and preserved extreme molecules}

This section will be devoted to proving the converse of Lemma 5.1.8; we will give an almost joint proof of the converse of both statements in the lemma. Let us introduce the notation

$$
\widetilde{M}=\left\{(x, y) \in M^{2}: x \neq y\right\}
$$

for the set of pairs of different points of $M$, with the topology inherited from $M^{2}$ (note that this notation was already used in the proof of Proposition 2.3.2, although no topology was assigned to $\widetilde{M}$ there).

Definition 5.2.1. The de Leeuw transform is the mapping $\Phi$ that assigns to a function $f: M \rightarrow \mathbb{R}$ the function $\Phi f: \widetilde{M} \rightarrow \mathbb{R}$ defined by

$$
\Phi f(x, y)=\frac{f(x)-f(y)}{d(x, y)} .
$$

We have not made any assumptions on $f$ in the definition above. If $f$ is continuous then $\Phi f$ is obviously continuous, too. Moreover, notice that $\|f\|_{L}=\|\Phi f\|_{\infty}$ by definition for any $f$. So in particular, if $f$ is Lipschitz then $\Phi f$ belongs to $C_{b}(\widetilde{M})$, the space of bounded continuous real-valued functions on $\widetilde{M}$, which can be identified with $C(\beta \widetilde{M})$. Thus we may regard $\Phi$ as a linear isometry from $\operatorname{Lip}_{0}(M)$ into $C(\beta \widetilde{M})$. Its adjoint operator $\Phi^{*}: \mathcal{M}(\beta \widetilde{M}) \rightarrow \mathcal{F}(M)^{* *}$ is therefore a quotient map, i.e. for every $\phi \in \mathcal{F}(M)^{* *}$ there exists a Radon measure $\mu \in \mathcal{M}(\beta \widetilde{M})$ such that $\Phi^{*} \mu=\phi$ and $\|\mu\|=\|\phi\|$. Note that $\Phi f(x, y)=\left\langle m_{x y}, f\right\rangle$ for any $f \in \operatorname{Lip}_{0}(M)$ and $x \neq y \in M$, so clearly $\Phi^{*} \delta_{(x, y)}=m_{x y}$.

\section{The sets $\mathcal{D}_{p q}$ and $\mathcal{E}_{p q}$}

For the rest of this section we will fix two distinct points $p, q$ in $M$ in order to analyze the extremality of the molecule $m_{p q}$. Let us consider the following sets:

$$
\begin{aligned}
\mathcal{D}_{p q} & =\left\{\zeta \in \beta \widetilde{M}:|\Phi f(\zeta)|=1 \text { for every } f \in S_{\operatorname{Lip}_{0}(M)} \text { such that } \Phi f(p, q)=1\right\} \\
\mathcal{E}_{p q} & =\left\{\phi \in B_{\mathcal{F}(M)^{* *}}:\langle f, \phi\rangle=1 \text { for every } f \in S_{\operatorname{Lip}_{0}(M)} \text { such that }\left\langle m_{p q}, f\right\rangle=1\right\}
\end{aligned}
$$


$\mathcal{D}_{p q}$ is a compact subset of $\beta \widetilde{M}$ and it always contains the points $(p, q)$ and $(q, p)$. Similarly, $\mathcal{E}_{p q}$ is a weak ${ }^{*}$-compact convex subset of $S_{\mathcal{F}(M)^{* *}}$ that always contains $m_{p q}$. Notice that $\mathcal{E}_{p q}$ is almost a "linear counterpart" of $\mathcal{D}_{p q}$ and, in fact, $\Phi^{*} \delta_{\zeta} \in$ $\mathcal{E}_{p q}$ for every $\zeta \in \mathcal{D}_{p q}{ }^{2}$

The relevance of these sets lies in the observation that $\mathcal{E}_{p q}$ is a face of $B_{\mathcal{F}(M)^{* *}}$; that is, if $\phi, \psi \in B_{\mathcal{F}(M)^{* *}}$ are such that

$$
t \phi+(1-t) \psi \in \mathcal{E}_{p q}
$$

for some $t \in(0,1)$, then $\phi, \psi \in \mathcal{E}_{p q}$. Indeed, let $f \in S_{\operatorname{Lip}_{0}(M)}$ be such that $\left\langle m_{p q}, f\right\rangle=1$. Then the inequalities

$$
1=\langle t \phi+(1-t) \psi, f\rangle=t\langle\phi, f\rangle+(1-t)\langle\psi, f\rangle \leq t+(1-t)=1
$$

hold and so $\langle\phi, f\rangle=\langle\psi, f\rangle=1$. Hence, in order to show that $m_{p q}$ is an extreme point of $B_{\mathcal{F}(M)}$, it suffices to show that $\operatorname{ext}\left(\mathcal{E}_{p q} \cap \mathcal{F}(M)\right)=\left\{m_{p q}\right\}$. And similarly, in order to show that $m_{p q}$ is a preserved extreme point, it is enough to show that $\operatorname{ext}\left(\mathcal{E}_{p q}\right)=\left\{m_{p q}\right\}$.

The following series of auxiliary results will be devoted to analyzing the properties of the sets $\mathcal{D}_{p q}$ and $\mathcal{E}_{p q}$ and the relationship between them. We start by showing that, informally speaking, $\mathcal{D}_{p q}$ is contained in $[p, q]_{\mathcal{U}} \times[p, q]_{\mathcal{U}}$ :

Lemma 5.2.2. For every $\zeta \in \mathcal{D}_{p q}$ there is a net $\left(x_{i}, y_{i}\right)$ in $\widetilde{M}$ that converges to $\zeta$ in $\beta \widetilde{M}$, such that $(p, q)_{x_{i}}$ and $(p, q)_{y_{i}}$ converge to 0 .

Proof. Let $\zeta \in \beta \widetilde{M}$, then there is a net $\left(x_{i}, y_{i}\right), i \in I$, in $\widetilde{M}$ that converges to $\zeta$ in $\beta \widetilde{M}$, and we may choose a subnet such that $\left(x_{i}\right)$ and $\left(y_{i}\right)$ converge to elements $\xi$ and $\eta$, respectively, in $M^{\mathcal{U}}$; call this subnet $\left(x_{i}, y_{i}\right)$ again. We want to prove that $\zeta \in \mathcal{D}_{p q}$ implies that $\xi, \eta \in[p, q]_{\mathcal{U}}$. We will do so by contradiction: assume without loss of generality that $\eta \notin[p, q]_{\mathcal{U}}$, and we will construct $f \in B_{\operatorname{Lip}_{0}(M)}$ such that $\Phi f(p, q)=1$ and $|\Phi f(\zeta)|<1$, thus concluding that $\zeta \notin \mathcal{D}_{p q}$.

We start by establishing the following fact:

Claim 1. Let $\left(z_{i}\right)$ be a net in $M$ that converges to $\vartheta \in M^{\mathcal{U}} \backslash[p, q]_{\mathcal{U}}$. Then we can replace $\left(z_{i}\right)$ with a subnet such that

$$
\inf _{i \in I} \frac{(p, q)_{z_{i}}}{d\left(z_{i}, q\right)}>0 .
$$

\footnotetext{
${ }^{2}$ The matching between $\mathcal{D}_{p q}$ and $\mathcal{E}_{p q}$ is not perfect due to the absolute value $|\Phi f(\zeta)|$ used in the definition of $\mathcal{D}_{p q}$. It is possible to remove it but this complicates the arguments, as it forces separate handling of points $(p, q) \in \widetilde{M}$ and their flipped versions $(q, p)$.
} 
Proof of Claim 1. Let $\left(z_{i}\right)$ be a net in $M$ such that $z_{i} \rightarrow \vartheta$. Since $(p, q)_{\vartheta}>0$, there is $\delta>0$ such that $(p, q)_{z_{i}} \geq \delta$ eventually. Now take a subnet such that either $d\left(z_{i}, q\right)$ is bounded by some $M<\infty$, or $d\left(z_{i}, q\right) \rightarrow \infty$. In the first case, we get $(p, q)_{z_{i}} / d\left(z_{i}, q\right) \geq \delta / M$ for all $i$. In the second case, we have $d\left(z_{i}, p\right) \geq$ $d\left(z_{i}, q\right)-d(p, q) \rightarrow \infty$ as well, and

$$
\limsup _{i} \frac{d\left(z_{i}, p\right)}{d\left(z_{i}, q\right)} \leq \limsup _{i} \frac{d\left(z_{i}, q\right)+d(q, p)}{d\left(z_{i}, q\right)}=1+\limsup _{i} \frac{d(p, q)}{d\left(z_{i}, q\right)}=1 .
$$

By symmetry in $p$ and $q$ we get $\lim _{i} d\left(z_{i}, p\right) / d\left(z_{i}, q\right)=1$. Hence

$$
\lim _{i} \frac{(p, q)_{z_{i}}}{d\left(z_{i}, q\right)}=\frac{1}{2}\left(1+\lim _{i} \frac{d\left(z_{i}, p\right)-d(p, q)}{d\left(z_{i}, q\right)}\right)=1,
$$

so we may take a subnet such that $(p, q)_{z_{i}} / d\left(z_{i}, q\right)>\frac{1}{2}$ for all $i$.

Apply Claim 1 with $\vartheta=\eta$ to replace $\left(x_{i}, y_{i}\right)$ with a subnet such that

$$
\inf _{i} \frac{(p, q)_{y_{i}}}{d\left(y_{i}, q\right)}>0 \text {. }
$$

Let us write $X=\left\{x_{i}: i \in I\right\}, Y=\left\{y_{i}: i \in I\right\}$ and $Z=\{p, q\} \cup X \cup Y$. We now split the rest of the proof into three possible cases that will be handled similarly but separately:

(1) $\xi \notin[p, q]_{\mathcal{U}}$.

(2) $\xi \in[p, q]_{\mathcal{U}} \backslash\{p, q\}$.

(3) $\xi \in\{p, q\}$.

Case 1: $\xi \notin[p, q]_{\mathcal{U}}$

By using Claim 1 again with $\vartheta=\xi$, we may assume that

$$
c=\min \left\{\frac{1}{2}, \inf _{i \in I} \frac{(p, q)_{x_{i}}}{d\left(x_{i}, q\right)}, \inf _{i \in I} \frac{(p, q)_{y_{i}}}{d\left(y_{i}, q\right)}\right\}
$$

is strictly positive. Define $g: Z \rightarrow \mathbb{R}$ by

$$
g(x)= \begin{cases}d(p, q) & \text { if } x=p \\ (1-c) \cdot d(x, q) & \text { if } x \in Z \backslash\{p\} .\end{cases}
$$

It is clear that $\Phi g(p, q)=1$ and $|\Phi g(x, y)| \leq 1-c$ for $x, y \in Z \backslash\{p\}$. For any $x \in Z \backslash\{p, q\}$ we have

$$
\begin{aligned}
& 1-\Phi g(p, x)=\frac{d(p, x)-g(p)+g(x)}{d(p, x)}=\frac{2(p, q)_{x}-c \cdot d(x, q)}{d(p, x)} \geq c \cdot \frac{d(x, q)}{d(p, x)}>0 \\
& 1+\Phi g(p, x)=\frac{d(p, x)+g(p)-g(x)}{d(p, x)}=\frac{2(x, q)_{p}+c \cdot d(x, q)}{d(p, x)} \geq c \cdot \frac{d(x, q)}{d(p, x)}>0
\end{aligned}
$$


so $-1<\Phi g(p, x)<1$, hence $\|g\|_{L}=1$. Now extend $g$ from $Z$ to $M$ using McShane's theorem and let $f=g-g(0)$. Then $f \in B_{\operatorname{Lip}_{0}(M)}, \Phi f(p, q)=1$, and $|\Phi f(\zeta)|=\lim _{i}\left|\Phi f\left(x_{i}, y_{i}\right)\right| \leq 1-c<1$, hence $\zeta \notin \mathcal{D}_{p q}$.

Case 2: $\xi \in[p, q]_{\mathcal{U}} \backslash\{p, q\}$

Since $p, q, \xi, \eta$ are all distinct, we may assume that $X$ and $Y$ are disjoint and do not contain $p$ or $q$. We require now a stronger version of Claim 1:

Claim 2. We may replace $\left(x_{i}, y_{i}\right), i \in I$, with a subnet such that

$$
\inf _{i \in I} \frac{(p, q)_{y_{i}}}{d\left(y_{i}, q\right)}>0, \inf _{i, j \in I} \frac{\left(x_{j}, q\right)_{y_{i}}}{d\left(y_{i}, q\right)}>0, \inf _{i \in I} \frac{d\left(y_{i}, q\right)}{d\left(y_{i}, p\right)}>0 \text { and } \inf _{i, j \in I} \frac{d\left(y_{i}, q\right)}{d\left(y_{i}, x_{j}\right)}>0 \text {. }
$$

Proof of Claim 2. By assumption we may choose a subnet such that $d\left(x_{i}, q\right)$ is bounded. We may further choose a subnet such that $d\left(y_{i}, q\right)$ is either bounded or goes to infinity. We divide the proof into these two cases.

Suppose first that we take a subnet such that $d\left(y_{i}, q\right) \leq C_{1}$ for some $C_{1}<\infty$ and all $i \in I$. The identity (5.1) implies in particular that

$$
\left(x_{j}, q\right)_{y_{i}} \geq(p, q)_{y_{i}}-(p, q)_{x_{j}}
$$

for any $i, j \in I$. Since $(p, q)_{\xi}=0$ and $(p, q)_{\eta}>0$, we may pass to a subnet such that $(p, q)_{y_{i}} \geq \delta$ and $(p, q)_{x_{i}} \leq \delta / 2$ for some $\delta>0$, so that

$$
\frac{\left(x_{j}, q\right)_{y_{i}}}{d\left(y_{i}, q\right)} \geq \frac{\delta}{2 C_{1}} \quad \text { and } \quad \frac{(p, q)_{y_{i}}}{d\left(y_{i}, q\right)} \geq \frac{\delta}{C_{1}}
$$

for all $i, j \in I$. Also $y_{i} \rightarrow \eta \neq q$, hence we may take a subnet such that $d\left(y_{i}, q\right) \geq C_{2}$ for some $C_{2}>0$ and all $i \in I$. If $C_{3}<\infty$ is such that $d\left(x_{i}, q\right) \leq C_{3}$ for all $i \in I$, we obtain

$$
\frac{d\left(y_{i}, q\right)}{d\left(y_{i}, x_{j}\right)} \geq \frac{d\left(y_{i}, q\right)}{d\left(y_{i}, q\right)+d\left(x_{j}, q\right)} \geq \frac{C_{2}}{C_{1}+C_{3}}
$$

for all $i, j \in I$, and similarly $d\left(y_{i}, q\right) / d\left(y_{i}, p\right) \geq C_{2} /\left(C_{1}+d(p, q)\right)$.

Now assume that we take a subnet such that $d\left(y_{i}, q\right) \rightarrow \infty$ instead. Mimicking the last part of the proof of Claim 1 we obtain

$$
\lim _{i} \frac{(p, q)_{y_{i}}}{d\left(y_{i}, q\right)}=1
$$

so we may take a subnet where $(p, q)_{y_{i}} / d\left(y_{i}, q\right)$ and $d\left(y_{i}, q\right) / d\left(y_{i}, p\right)$ are bounded below by a positive constant. Also, since $d\left(x_{i}, q\right)$ is bounded, we may choose a further subnet such that $d\left(x_{j}, q\right) / d\left(y_{i}, q\right) \leq 1 / 2$ for all $i, j$, and then

$$
\frac{\left(x_{j}, q\right)_{y_{i}}}{d\left(y_{i}, q\right)}=\frac{1}{2}\left(1+\frac{d\left(y_{i}, x_{j}\right)-d\left(x_{j}, q\right)}{d\left(y_{i}, q\right)}\right) \geq \frac{1}{2}\left(\frac{1}{2}+\frac{d\left(y_{i}, x_{j}\right)}{d\left(y_{i}, q\right)}\right) \geq \frac{1}{4} .
$$


Finally,

$$
\frac{d\left(y_{i}, x_{j}\right)}{d\left(y_{i}, q\right)} \leq 1+\frac{d\left(x_{j}, q\right)}{d\left(y_{i}, q\right)} \leq \frac{3}{2}
$$

and so $d\left(y_{i}, q\right) / d\left(y_{i}, x_{j}\right) \geq 2 / 3$ for all $i, j$.

Now we continue with the main proof. Using Claim 2, replace $\left(x_{i}, y_{i}\right)$ with a subnet such that

$$
c=\min \left\{\frac{1}{2}, \inf _{i, j \in I} \frac{\left(x_{j}, q\right)_{y_{i}}}{d\left(y_{i}, q\right)}, \inf _{i \in I} \frac{(p, q)_{y_{i}}}{d\left(y_{i}, q\right)}\right\}
$$

and

$$
\delta=\min \left\{1, \inf _{i, j \in I} \frac{d\left(y_{i}, q\right)}{d\left(y_{i}, x_{j}\right)}, \inf _{i \in I} \frac{d\left(y_{i}, q\right)}{d\left(y_{i}, p\right)}\right\} .
$$

are positive. Define $g: Z \rightarrow \mathbb{R}$ by

$$
g(z)= \begin{cases}d(z, q) & \text { if } z \in Z \backslash Y \\ (1-c) \cdot d(z, q) & \text { if } z \in Y\end{cases}
$$

It is clear that $\Phi g(p, q)=1,|\Phi g(x, y)| \leq 1$ for $x, y \in Z \backslash Y$ and $|\Phi g(x, y)| \leq 1-c$ for $x, y \in Y$. Moreover, if $y \in Y$ then $\Phi g(y, q)=1-c$, and for any $x \in X \cup\{p\}$ we have

$$
\begin{aligned}
& 1+\Phi g(y, x)=\frac{2(x, q)_{y}-c \cdot d(y, q)}{d(y, x)} \geq c \cdot \frac{d(y, q)}{d(y, x)} \geq c \delta, \\
& 1-\Phi g(y, x)=\frac{2(y, q)_{x}+c \cdot d(y, q)}{d(y, x)} \geq c \cdot \frac{d(y, q)}{d(y, x)} \geq c \delta,
\end{aligned}
$$

so $|\Phi g(y, x)| \leq 1-c \delta$. We conclude that $\|g\|_{L}=1$. Now extend $g$ from $Z$ to $M$ and let $f=g-g(0)$. Then $f \in B_{\operatorname{Lip}_{0}(M)}, \Phi f(p, q)=1$, and $|\Phi f(\zeta)|=$ $\lim _{i}\left|\Phi f\left(x_{i}, y_{i}\right)\right| \leq 1-c \delta<1$, hence $\zeta \notin \mathcal{D}_{p q}$.

Case 3: $\xi \in\{p, q\}$

Assume that $\xi=q$ without loss of generality. Then we may use a simplified version of the construction in Case 1: let $Z=\{p, q\} \cup Y$ and define $g: Z \rightarrow \mathbb{R}$ by

$$
g(x)= \begin{cases}d(p, q) & \text { if } x=p \\ (1-c) \cdot d(x, q) & \text { if } x \in Z \backslash\{p\} .\end{cases}
$$

where

$$
c=\min \left\{\frac{1}{2}, \inf _{i \in I} \frac{(p, q)_{y_{i}}}{d\left(y_{i}, q\right)}\right\}>0,
$$

then extend it to $M$ and take $f=g-g(0)$. Then we again obtain $f \in B_{\operatorname{Lip}_{0}(M)}$, $\Phi f(p, q)=1$ and $|\Phi f(\zeta)| \leq 1-c$; we omit the details. This concludes the proof of Lemma 5.2.2. 
The next result says that if $\mu \in \mathcal{M}(\beta \widetilde{M})$ is such that $\Phi^{*} \mu \in \mathcal{E}_{p q}$, then $\mu$ must be almost concentrated on $\mathcal{D}_{p q}$.

Lemma 5.2.3. Let $U \subset \beta \widetilde{M}$ be an open neighborhood of $\mathcal{D}_{p q}$. Then there is a finite constant $C_{U}$, depending on $U$, such that

$$
|\mu|(\beta \widetilde{M} \backslash U) \leq C_{U} \cdot(\|\mu\|-1)
$$

for any measure $\mu \in \mathcal{M}(\beta \widetilde{M})$ such that $\Phi^{*} \mu \in \mathcal{E}_{p q}$.

Proof. Denote $K=\beta \widetilde{M} \backslash U$, which is a compact set. For any $\zeta \in K$ we have $\zeta \notin \mathcal{D}_{p q}$, so there is $f_{\zeta} \in B_{\operatorname{Lip}_{0}(M)}$ such that $\Phi f_{\zeta}(p, q)=1$ and $\left|\Phi f_{\zeta}(\zeta)\right|<1$ and, since $\Phi f_{\zeta}$ is continuous, there are $c_{\zeta} \in(0,1)$ and an open neighborhood $V_{\zeta}$ of $\zeta$ such that $\left|\Phi f_{\zeta}\left(\zeta^{\prime}\right)\right| \leq c_{\zeta}$ for every $\zeta^{\prime} \in V_{\zeta}$. If $\mu \in \mathcal{M}(\beta \widetilde{M})$ is any measure such that $\Phi^{*} \mu \in \mathcal{E}_{p q}$, then we have

$$
\begin{aligned}
1=\left\langle f_{\zeta}, \Phi^{*} \mu\right\rangle=\int_{\beta \widetilde{M}}\left(\Phi f_{\zeta}\right) d \mu & =\int_{V_{\zeta}}\left(\Phi f_{\zeta}\right) d \mu+\int_{\beta \widetilde{M} \backslash V_{\zeta}}\left(\Phi f_{\zeta}\right) d \mu \\
& \leq c_{\zeta}|\mu|\left(V_{\zeta}\right)+|\mu|\left(\beta \widetilde{M} \backslash V_{\zeta}\right) \\
& =\|\mu\|-\left(1-c_{\zeta}\right)|\mu|\left(V_{\zeta}\right)
\end{aligned}
$$

hence

$$
|\mu|\left(V_{\zeta}\right) \leq \frac{\|\mu\|-1}{1-c_{\zeta}}
$$

Now, $\left\{V_{\zeta}: \zeta \in K\right\}$ is an open cover of $K$, so it admits a finite subcover $K \subset$ $\bigcup_{j=1}^{n} V_{\zeta_{j}}$. Thus, for any $\mu \in \mathcal{M}\left((\beta \widetilde{M})\right.$ such that $\Phi^{*} \mu \in \mathcal{E}_{p q}$ we have

$$
|\mu|(K) \leq \sum_{j=1}^{n}|\mu|\left(V_{\zeta_{j}}\right) \leq C_{U} \cdot(\|\mu\|-1),
$$

where $C_{U}=\sum_{j=1}^{n}\left(1-c_{\zeta_{j}}\right)^{-1}<\infty$.

With the previous lemma at hand, we can start tightening the net around the elements of $\mathcal{E}_{p q}$. Let $\pi_{1}, \pi_{2}: \widetilde{M} \rightarrow M$ be the projection mappings given by $\pi_{1}(x, y)=x$ and $\pi_{2}(x, y)=y$. For a set $A \subset \widetilde{M}$, denote $\pi(A)=\pi_{1}(A) \cup \pi_{2}(A)$, i.e. $\pi(A)$ will be the set of points of $M$ appearing as either coordinate of an element of $A$.

Lemma 5.2.4. Let $U \subset \beta \widetilde{M}$ be an open neighborhood of $\mathcal{D}_{p q}$. Then for every $m \in \mathcal{E}_{p q} \cap \mathcal{F}(M)$ we have $\operatorname{supp}(m) \subset \overline{\pi(U \cap \widetilde{M})}$. 
Proof. Let $A=\overline{\pi(U \cap \widetilde{M})}$ and fix $k \in \mathbb{N}$. By Proposition 2.3.2, $m$ admits a representation $m=\sum_{n=1}^{\infty} a_{n} m_{p_{n} q_{n}}$ where $\left(p_{n}, q_{n}\right) \in \widetilde{M}$ for all $n \in \mathbb{N}$ and $\sum_{n=1}^{\infty}\left|a_{n}\right| \leq 1+1 / k$. Let $\mu=\sum_{n=1}^{\infty} a_{n} \delta_{\left(p_{n}, q_{n}\right)}$, then $\mu$ belongs to $\mathcal{M}(\beta \widetilde{M})$ as the series is absolutely convergent, $\|\mu\| \leq \sum_{n=1}^{\infty}\left|a_{n}\right| \leq 1+1 / k$, and clearly $\Phi^{*} \mu=m$.

Now let $I=\left\{n \in \mathbb{N}:\left(p_{n}, q_{n}\right) \in U\right\}$ and $m_{k}=\sum_{n \in I} a_{n} m_{p_{n} q_{n}}$. Notice that $p_{n}, q_{n} \in$ $A$ for each $n \in I$, hence $\operatorname{supp}\left(m_{k}\right) \subset A$. Denote $K=\beta \widetilde{M} \backslash U$ and let $C_{U}$ be the constant assigned to $U$ by Lemma 5.2.3. For each $f \in \operatorname{Lip}_{0}(M)$ we have

$$
\begin{aligned}
\left\langle m-m_{k}, f\right\rangle & =\sum_{n \notin I} a_{n} \Phi f\left(p_{n}, q_{n}\right)=\sum_{\left\{n \in \mathbb{N}:\left(p_{n}, q_{n}\right) \in K\right\}} a_{n} \Phi f\left(p_{n}, q_{n}\right) \\
& =\int_{\beta \widetilde{M}}(\Phi f) \cdot \chi_{K} d \mu=\int_{K}(\Phi f) d \mu
\end{aligned}
$$

and it follows that

$$
\left|\left\langle m-m_{k}, f\right\rangle\right|=\left|\int_{K}(\Phi f) d \mu\right| \leq\|\Phi f\|_{\infty} \cdot|\mu|(K) \leq\|f\|_{L} \cdot C_{U} / k
$$

and $\left\|m-m_{k}\right\| \leq C_{U} / k$. Hence, if $k \rightarrow \infty$ then $m_{k} \rightarrow m$. Since $\operatorname{supp}\left(m_{k}\right) \subset A$ for any $k$ we conclude that $\operatorname{supp}(m) \subset A$.

Finally we obtain that all weak* continuous elements of $\mathcal{E}_{p q}$ must be supported on the metric segment $[p, q]$. Crucially, the intersection theorem is required to reach this conclusion.

Lemma 5.2.5. For every $m \in \mathcal{E}_{p q} \cap \mathcal{F}(M)$ we have $\operatorname{supp}(m) \subset[p, q]$.

Proof. Let $\varphi: \widetilde{M} \rightarrow[0, \infty)$ be the continuous function defined by

$$
\varphi(x, y)=\max \left\{(p, q)_{x},(p, q)_{y}\right\}
$$

and extend $\varphi$ to a continuous function $\varphi: \beta \widetilde{M} \rightarrow[0, \infty]$. Consider the sets

$$
S_{n}=\left\{x \in M:(p, q)_{x} \leq \frac{1}{n}\right\}
$$

and

$$
U_{n}=\left\{\zeta \in \beta \widetilde{M}: \varphi(\zeta)<\frac{1}{n}\right\}
$$

for $n \in \mathbb{N}$. Notice that $S_{n}$ is closed, $U_{n}$ is open in $\beta \widetilde{M}$, and $\pi\left(U_{n} \cap \widetilde{M}\right) \subset S_{n}$ by definition. For each $n$, Lemma 5.2.2 implies that $\mathcal{D}_{p q} \subset U_{n}$, and applying Lemma 5.2.4 we conclude that $\operatorname{supp}(m) \subset S_{n}$. Thus the intersection theorem yields

$$
\operatorname{supp}(m) \subset \bigcap_{n=1}^{\infty} S_{n}=[p, q]
$$

as claimed. 


\section{Extreme molecules}

We can now obtain the main result in this chapter as an immediate consequence of Lemma 5.2.5:

Theorem 5.2.6. Let $p, q$ be different points of $M$. Then $m_{p q} \in \operatorname{ext} B_{\mathcal{F}(M)}$ if and only if $[p, q]$ is trivial.

Proof. One direction is given by Lemma 5.1.8(a). For the other one, suppose that $[p, q]=\{p, q\}$. We already remarked that it suffices to show that

$$
\operatorname{ext}\left(\mathcal{E}_{p q} \cap \mathcal{F}(M)\right)=\left\{m_{p q}\right\}
$$

In fact, something stronger holds: we have $\mathcal{E}_{p q} \cap \mathcal{F}(M)=\left\{m_{p q}\right\}$. Indeed, if $m \in \mathcal{E}_{p q} \cap \mathcal{F}(M)$ then $\operatorname{supp}(m) \subset[p, q]=\{p, q\}$ by Lemma 5.2.5, hence $m=$ $a \delta(p)+b \delta(q)$ for some $a, b \in \mathbb{R}$, and it is straightforward to check that $m \in \mathcal{E}_{p q}$ if and only if $m=m_{p q}$.

In fact, this result characterizes all extreme points of $B_{\mathcal{F}(M)}$ with finite support:

Corollary 5.2.7. Let $m \in \operatorname{span} \delta(M)$. Then $m \in \operatorname{ext} B_{\mathcal{F}(M)}$ if and only if $m=m_{p q}$ where $p, q$ are different points of $M$ such that $[p, q]$ is trivial.

Proof. Let $K=\operatorname{supp}(m)$. If $m \in \operatorname{ext} B_{\mathcal{F}(M)}$ then also $m \in \operatorname{ext} B_{\mathcal{F}_{M}(K)}$. But $\mathcal{F}_{M}(K)$ is finite dimensional, hence every extreme point of the unit ball is preserved extreme and thus a molecule by Theorem 5.1.2. Now it suffices to apply Theorem 5.2.6.

An application of Theorem 5.2.6 allows us to show that there exists a complete metric space $M$ such that all of its elementary molecules are extreme but none of them are preserved. This provides a rather strong example of the fact that the implication (preserved extreme $\Rightarrow$ extreme) cannot be reversed.

Example 5.2.8. Let $M$ be the space described in [35, Example 2.4]. It is shown there to have the following properties:

(i) it is a closed subset of a strictly convex Banach space,

(ii) it contains no nontrivial linear segments,

(iii) it is "almost metrically convex", i.e. a length space.

It follows from (i) and (ii) that $M$ contains no nontrivial metric segments, hence Theorem 5.2.6 implies that all elementary molecules are extreme points of $B_{\mathcal{F}(M)}$. However, by (iii) and [24, Proposition 5.9], $B_{\mathcal{F}(M)}$ has no preserved extreme point. 


\section{Preserved extreme points}

The other main result, the characterization of preserved extreme points, follows from the particular case of Lemma 5.2.3 where we take $\|\mu\|=1$.

Theorem 5.2.9. The preserved extreme points of $B_{\mathcal{F}(M)}$ are exactly the molecules $m_{p q}$ for $p \neq q \in M$ such that $[p, q]_{\mathcal{U}}$ is trivial.

Recall that the geometric characterization of this condition within the metric of $M$ is given in Lemma 5.1.9.

Proof. One implication is given by Theorem 5.1.2 and Lemma 5.1.8(b). For the other one, assume that $[p, q]_{\mathcal{U}}=\{p, q\}$. Since $\Phi^{*}$ is a quotient map, for any $\phi \in \mathcal{E}_{p q}$ there is $\mu \in S_{\mathcal{M}(\beta \widetilde{M})}$ such that $\Phi^{*} \mu=\phi$. If $U \subset \beta \widetilde{M}$ is any open neighborhood of $\mathcal{D}_{p q}$ then $|\mu|(\beta \widetilde{M} \backslash U)=0$ by Lemma 5.2 .3 , hence

$$
|\mu|\left(\beta \widetilde{M} \backslash \mathcal{D}_{p q}\right)=\sup \left\{|\mu|(\beta \widetilde{M} \backslash U): U \supset \mathcal{D}_{p q} \text { open }\right\}=0
$$

by regularity. Thus $\mu$ is concentrated on $\mathcal{D}_{p q}$. But $[p, q]_{\mathcal{U}}$ is trivial, so Lemma 5.2.2 implies that

$$
\mathcal{D}_{p q}=\{(p, q),(q, p)\} \cup A_{p} \cup A_{q}
$$

where $A_{p}$ (resp. $A_{q}$ ) is a (possibly empty) subset of $\beta \widetilde{M}$ all whose elements are limits of nets $\left(x_{i}, y_{i}\right)$ in $\widetilde{M}$ such that $\left(x_{i}\right)$ and $\left(y_{i}\right)$ converge to $p$ (resp. $q$ ). Hence

$$
\mu=a \delta_{(p, q)}+b \delta_{(q, p)}+\mu^{\prime}
$$

for some $a, b \in \mathbb{R}$ and some $\mu^{\prime} \in \mathcal{M}(\beta \widetilde{M})$ that is concentrated on $A_{p} \cup A_{q}$. In particular, we have

$$
1=|\mu|(\beta \widetilde{M})=|a|+|b|+\left\|\mu^{\prime}\right\| .
$$

Suppose that $m_{p q}=\frac{1}{2}\left(\phi_{1}+\phi_{2}\right)$ for some $\phi_{i} \in S_{\mathcal{F}(M)^{* *}}$. It was already observed that $\phi_{i} \in \mathcal{E}_{p q}$ in that case, so we have $\phi_{i}=\Phi^{*} \mu_{i}$ where $\mu_{i} \in S_{\mathcal{M}(\beta \widetilde{M})}$ has the form $\mu_{i}=a_{i} \delta_{(p, q)}+b_{i} \delta_{(q, p)}+\mu_{i}^{\prime}$ and $\mu_{i}^{\prime}$ is concentrated on $A_{p} \cup A_{q}$.

Now let $U$ and $V$ be neighborhoods of $p$ and $q$, respectively, such that $d(U, V)>0$. Use McShane's theorem to build a function $g \in \operatorname{Lip}(M)$ such that $g=d(p, q)$ on $U$ and $g=0$ on $V$, then let $f=g-g(0)$. Clearly $f \in \operatorname{Lip}_{0}(M)$ and $\Phi f(p, q)=1$. Moreover, $\Phi f=0$ on $A_{p}$ : indeed, for any $\zeta \in A_{p}$ choose a net $\left(x_{i}, y_{i}\right)$ in $\widetilde{M}$ that converges to $\zeta$, then $x_{i}, y_{i}$ are eventually in $U$, hence $\Phi f\left(x_{i}, y_{i}\right)=0$ eventually and 
so $\Phi f(\zeta)=0$. Similarly $\Phi f=0$ on $A_{q}$. But then we get

$$
\begin{aligned}
1=\left\langle m_{p q}, f\right\rangle & =\frac{1}{2}\left\langle f, \phi_{1}+\phi_{2}\right\rangle \\
& =\frac{1}{2}\left(\left(a_{1}-b_{1}+a_{2}-b_{2}\right)\left\langle m_{p q}, f\right\rangle+\int_{\beta \widetilde{M}}(\Phi f) d\left(\mu_{1}^{\prime}+\mu_{2}^{\prime}\right)\right) \\
& =\frac{1}{2}\left(a_{1}-b_{1}+a_{2}-b_{2}\right)
\end{aligned}
$$

and it follows that

$$
\begin{aligned}
2=a_{1}-b_{1}+a_{2}-b_{2} & \leq\left|a_{1}\right|+\left|b_{1}\right|+\left|a_{2}\right|+\left|b_{2}\right| \\
& \leq\left|a_{1}\right|+\left|b_{1}\right|+|| \mu_{1}^{\prime}||+\left|a_{2}\right|+\left|b_{2}\right|+\left\|\mu_{2}^{\prime}\right\|=2 .
\end{aligned}
$$

Therefore all the inequalities are in fact equalities, in particular $\left\|\mu_{1}^{\prime}\right\|=\left\|\mu_{2}^{\prime}\right\|=0$ and thus $\phi_{i}=\left(a_{i}-b_{i}\right) m_{p q}$ are scalar multiples of $m_{p q}$. It is then immediate that $\phi_{1}=\phi_{2}=m_{p q}$ and $m_{p q} \in \operatorname{ext} B_{\mathcal{F}(M)^{* *}}$ as we wanted to prove.

When $M$ is compact, or more generally when it is a Heine-Borel space, it is easy to see that $[p, q]_{\mathcal{U}}=[p, q]$. This implies easily that $\operatorname{Mol}(M)$ cannot contain unpreserved extreme points of $B_{\mathcal{F}(M)}$ :

Corollary 5.2.10. Suppose that $M$ is a Heine-Borel space and let $p, q$ be different points of $M$. Then the following are equivalent:

(i) $m_{p q}$ is a preserved extreme point of $B_{\mathcal{F}(M)}$,

(ii) $m_{p q}$ is an extreme point of $B_{\mathcal{F}(M)}$,

(iii) $[p, q]$ is trivial.

Proof. (i) $\Rightarrow$ (ii) is trivial and (ii) $\Rightarrow$ (iii) is Lemma 5.1.8(a). Now suppose (i) fails, then by Theorem 5.2.9 there is $\xi \in[p, q]_{\mathcal{U}} \backslash\{p, q\}$. Let $\left(x_{i}\right)$ be a net in $M$ that converges to $\xi$. Since $d\left(p, x_{i}\right)+d\left(q, x_{i}\right)$ converges to $d(p, q)$, we may assume that $\left(x_{i}\right)$ is bounded. The Heine-Borel property then implies that $\left(x_{i}\right)$ has a cluster point $x \in M$ that is different from $p, q$ and clearly $d(p, x)+d(q, x)=d(p, q)$. Thus (iii) also fails.

We remark that Corollary 5.2.10 is no longer true when we remove the hypothesis that $M$ is Heine-Borel. Simple counterexamples may be constructed using Theorem 5.2.9, such as the following one:

Example 5.2.11. Let $M \subset c_{0}$ consist of $0, p=2 e_{1}$, and $q_{n}=e_{1}+\left(1+\frac{1}{n}\right) e_{n}$ for $n \geq 2$. Since $d\left(q_{n}, q_{m}\right)>1$ for different $n, m \geq 2$, the sequence $\left\{q_{n}\right\}$ has no cluster point in $M$, and $M$ is not compact. Also $(p, 0)_{q_{n}}=\frac{1}{n}$, so the segment $[0, p]$ is trivial. However, if $\xi$ is a cluster point of $q_{n}$ in $M^{\mathcal{U}}$ then $(p, 0)_{\xi}=0$, hence $m_{p 0}$ is 
not a preserved extreme point by Theorem 5.2.9. However it is an extreme point by Theorem 5.2.6.

A metric space is called concave when all of its metric segments are trivial, i.e. when no triple of different points of $M$ is metrically aligned. As an immediate consequence of Corollary 5.2.10 we obtain the following equivalence, which solves an open problem posed by Weaver in $\left[52\right.$, p. 53]..$^{3}$

Corollary 5.2.12. Suppose that $M$ is a Heine-Borel space. Then $M$ is concave if and only if every elementary molecule of $M$ is a preserved extreme point of $B_{\mathcal{F}(M)}$.

Classical examples of concave spaces are given by Hölder spaces $M^{\theta}$, which are constructed from metric spaces $(M, d)$ by equipping them with the metric $d^{\theta}$ where $\theta \in(0,1)$. Indeed, for distinct $p, q, r \in M$ we have

$$
d(p, q)^{\theta} \leq(d(p, r)+d(r, q))^{\theta}<d(p, r)^{\theta}+d(r, q)^{\theta}
$$

so that no set of three distinct points can be metrically aligned in $M^{\theta}$. We remark that not all compact concave spaces are Hölder spaces, as shown by the following example:

Example 5.2.13. Consider strictly decreasing sequences $\lambda_{n} \rightarrow 1$ and $a_{n} \rightarrow 0$, with $a_{1}<1$. Then $a_{n}^{\lambda_{n}}+\left(1-a_{n}\right)^{\lambda_{n}}<\left(a_{n}+1-a_{n}\right)^{\lambda_{n}}=1$, so we can choose positive $b_{n} \rightarrow 0$ such that

$$
\left(a_{n}^{2}+b_{n}^{2}\right)^{\lambda_{n} / 2}+\left(\left(1-a_{n}\right)^{2}+b_{n}^{2}\right)^{\lambda_{n} / 2}<1 .
$$

Note that the terms in parentheses are all smaller than 1 . Let $M$ be the subset of $\ell_{2}$ consisting of $0, e_{1}$, and $r_{n}=a_{n} e_{1}+b_{n} e_{n}$ for $n \geq 2$. Then $M$ is compact because $r_{n} \rightarrow 0$, and any triple of distinct points of $M$ spans an affine subspace of $\ell_{2}$ of dimension 2 so they cannot be metrically aligned because $\ell_{2}$ is strictly convex; hence $M$ is concave. However $M$ cannot be $\theta$-Hölder for any $\theta \in(0,1)$. Indeed, suppose there was a metric $d$ on $M$ such that $\|x-y\|_{2}=d(x, y)^{\theta}$ for any $x, y \in M$, and choose $n$ such that $\lambda_{n}<1 / \theta$. Then

$$
\begin{aligned}
d\left(0, r_{n}\right)+d\left(r_{n}, e_{1}\right) & =\left\|r_{n}\right\|_{2}^{1 / \theta}+\left\|e_{1}-r_{n}\right\|_{2}^{1 / \theta} \\
& =\left(a_{n}^{2}+b_{n}^{2}\right)^{1 / 2 \theta}+\left(\left(1-a_{n}\right)^{2}+b_{n}^{2}\right)^{1 / 2 \theta} \\
& <\left(a_{n}^{2}+b_{n}^{2}\right)^{\lambda_{n} / 2}+\left(\left(1-a_{n}\right)^{2}+b_{n}^{2}\right)^{\lambda_{n} / 2} \\
& <1=d\left(0, e_{1}\right)
\end{aligned}
$$

violating the triangle inequality.

\footnotetext{
${ }^{3} \mathrm{~A}$ different proof, which appears in [53], was found independently by Weaver himself.
} 


\subsection{Other extremal elements}

We will end this chapter by collecting a few additional results on the extremal structure of $\mathcal{F}(M)$. Namely, we will characterize molecules that are exposed points of $B_{\mathcal{F}(M)}$, extreme points of $B_{\mathcal{F}(M)}$ that can be written as a positive functional plus a finitely supported element, and extreme points of the positive unit ball $B_{\mathcal{F}(M)}^{+}$.

\section{Exposed molecules}

We will start by showing that every molecule that is an extreme point of $B_{\mathcal{F}(M)}$ is also exposed. The proof uses completely different techniques as that of Theorem 5.2.6 (although both require the use of supports or the intersection theorem), and in fact it provides a shorter proof of Theorem 5.2.6. However, it relies on previous knowledge of a candidate exposing functional for $m_{p q}$ : it is the function $f_{p q}$ defined by

$$
f_{p q}(x)=\frac{d(p, q)}{2} \frac{d(x, q)-d(x, p)}{d(x, q)+d(x, p)}+C
$$

for $x \in M$, where the constant $C$ is chosen so that $f_{p q}(0)=0$. It is clear that $f_{p q} \in S_{\operatorname{Lip}_{0}(M)}$ and that $\left\langle m_{p q}, f_{p q}\right\rangle=1$. This function was introduced and studied in $[35,36]$, where the following property was proved:

Lemma 5.3.1. Let $p \neq q \in M$. If $u \neq v \in M$ and $\varepsilon \in[0,1]$ are such that $\left\langle m_{u v}, f_{p q}\right\rangle \geq 1-\varepsilon$, then $u, v \in[p, q]_{\varepsilon}$ where

$$
[p, q]_{\varepsilon}=\left\{x \in M: d(p, x)+d(x, q) \leq \frac{1}{1-\varepsilon} d(p, q)\right\} .
$$

Using the intersection theorem, we can now prove the next strengthening of Lemma 5.3.1.

Lemma 5.3.2. Let $p \neq q \in M$. If $m \in B_{\mathcal{F}(M)}$ is such that $\left\langle m, f_{p q}\right\rangle=1$, then $\operatorname{supp}(m) \subset[p, q]$.

Proof. Let $\delta, \varepsilon>0$. It follows from Proposition 2.3.2 that we may find an expression $m=\sum_{n=1}^{\infty} a_{n} m_{x_{n} y_{n}}$ where $x_{n} \neq y_{n} \in M$ for $n \in \mathbb{N}$ and $\sum_{n}\left|a_{n}\right|<1+\delta \varepsilon$. 
Let $I=\left\{n \in \mathbb{N}:\left|\left\langle m_{x_{n} y_{n}}, f_{p q}\right\rangle\right| \geq 1-\varepsilon\right\}$. Then

$$
\begin{aligned}
1=\left\langle m, f_{p q}\right\rangle & =\sum_{n=1}^{\infty} a_{n}\left\langle m_{x_{n} y_{n}}, f_{p q}\right\rangle \\
& =\sum_{n \in I} a_{n}\left\langle m_{x_{n} y_{n}}, f_{p q}\right\rangle+\sum_{n \in \mathbb{N} \backslash I} a_{n}\left\langle m_{x_{n} y_{n}}, f_{p q}\right\rangle \\
& \leq \sum_{n \in I}\left|a_{n}\right|+(1-\varepsilon) \sum_{n \in \mathbb{N} \backslash I}\left|a_{n}\right| \\
& <1+\delta \varepsilon-\varepsilon \sum_{n \in \mathbb{N} \backslash I}\left|a_{n}\right| .
\end{aligned}
$$

Hence $\sum_{n \in \mathbb{N} \backslash I}\left|a_{n}\right|<\delta$, and it follows that

$$
\left\|m-\sum_{n \in I} a_{n} m_{x_{n} y_{n}}\right\|=\left\|\sum_{n \in \mathbb{N} \backslash I} a_{n} m_{x_{n} y_{n}}\right\| \leq \sum_{n \in \mathbb{N} \backslash I}\left|a_{n}\right|<\delta .
$$

Notice that $x_{n}, y_{n} \in[p, q]_{\varepsilon}$ if $n \in I$, by Lemma 5.3.1. Thus $m$ may be approximated by elements of $\mathcal{F}(M)$ supported on $[p, q]_{\varepsilon}$, hence $\operatorname{supp}(m) \subset[p, q]_{\varepsilon}$. But $\varepsilon>0$ was also arbitrary, $\operatorname{so} \operatorname{supp}(m) \subset \bigcap_{\varepsilon>0}[p, q]_{\varepsilon}=[p, q]$.

Theorem 5.3.3. Let $p \neq q \in M$. Then the following are equivalent:

(i) $m_{p q}$ is an extreme point of $B_{\mathcal{F}(M)}$,

(ii) $m_{p q}$ is an exposed point of $B_{\mathcal{F}(M)}$,

(iii) $[p, q]$ is trivial.

Proof. (ii) $\Rightarrow$ (i) is trivial, and (i) $\Rightarrow$ (iii) is Lemma 5.1.8(a). Now assume (iii) and let us check that $m_{p q}$ is exposed by $f_{p q}$. Indeed, $f_{p q} \in S_{\operatorname{Lip}_{0}(M)}$ and $\left\langle m_{p q}, f_{p q}\right\rangle=1$. If $m \in B_{\mathcal{F}(M)}$ is such that $\left\langle m, f_{p q}\right\rangle=1$, then $\operatorname{supp}(m) \subset[p, q]=\{p, q\}$ by Lemma 5.3.2. Thus $m=a \delta(p)+b \delta(q)$ for some $a, b \in \mathbb{R}$, and a straightforward computation shows that only $m=m_{p q}$ satisfies the constraints $\|m\| \leq 1$ and $\left\langle m, f_{p q}\right\rangle=1$. This proves (ii).

\section{Extreme points of the positive ball}

Let us now consider the extreme points of $B_{\mathcal{F}(M)}^{+}$. The following theorem completely characterizes them, and it moreover shows that all of them are actually preserved.

Theorem 5.3.4. The extreme points of $B_{\mathcal{F}(M)}^{+}$are precisely the normalized evaluation functionals, i.e. 0 and $\delta(x) / d(x, 0)$ for $x \in M \backslash\{0\}$. Moreover, all of them are preserved. 
Proof. First we will show that all extreme points are normalized evaluation functionals, or equivalently, their support does not contain more than one point. Let $m \in B_{\mathcal{F}(M)}^{+}$with $\|m\|=1$ be such that $\operatorname{supp}(m)$ contains at least two points $a$ and $b$; we will show that $m$ is not an extreme point of $B_{\mathcal{F}(M)}^{+}$. We may assume $a, b \neq 0$ : if $0 \in \operatorname{supp}(m)$ then 0 must be an accumulation point of $\operatorname{supp}(m)$, hence $\operatorname{supp}(m)$ is infinite.

Denote $r=d(a, b) / 3$. Let $h \in \operatorname{Lip}(M)$ be defined by

$$
h(x)=0 \vee\left(1-\frac{d(x, B(a, r))}{r}\right)
$$

for $x \in M$, so that $0 \leq h \leq 1, h \uparrow_{B(a, r)}=1, h \uparrow_{B(b, r)}=0$, and $\operatorname{supp}(h)$ is bounded. Notice that $m \circ W_{h} \neq 0$. Indeed, using McShane's theorem choose $f \in \operatorname{Lip}_{0}(M)$ such that $f \geq 0, f(a)=1$ and $\operatorname{supp}(f) \subset B(a, r)$, then $\left\langle m \circ W_{h}, f\right\rangle=\langle m, f\rangle>0$ by (3.13) and Proposition 3.3.12(a) because $a \in \operatorname{supp}(m)$. A similar argument using a function supported on $B(b, r)$ shows that $m \circ W_{1-h} \neq 0$. Since $h$ and $1-h$ are both positive, so are $m \circ W_{h}$ and $m \circ W_{1-h}=m-m \circ W_{h}$ and thus

$$
\left\|m \circ W_{h}\right\|+\left\|m \circ W_{1-h}\right\|=\left\|m \circ W_{h}+m \circ W_{1-h}\right\|=\|m\|=1
$$

by Proposition 2.3.3. But then

$$
m=\left\|m \circ W_{h}\right\| \frac{m \circ W_{h}}{\left\|m \circ W_{h}\right\|}+\left\|m \circ W_{1-h}\right\| \frac{m \circ W_{1-h}}{\left\|m \circ W_{1-h}\right\|}
$$

is a nontrivial convex combination of elements of $B_{\mathcal{F}(M)}^{+}$, as was to be shown.

Now let $x \in M$ and $m=\delta(x) / d(x, 0)$ if $x \neq 0$ or $m=0$ if $x=0$; we will show that $m$ is really a preserved extreme point of $B_{\mathcal{F}(M)}^{+}$. Suppose that $m=\frac{1}{2}(\phi+\psi)$

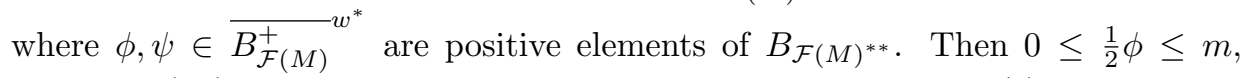
so $\phi \in \mathcal{F}(M)$ by Lemma 4.3.14. Moreover, Proposition 3.3.12(c) implies that $\operatorname{supp}(\phi) \subset\{x\}$. Using Proposition 3.3.8, this is enough to conclude that $\phi=\psi=$ $m$, which finishes the proof.

Let us note that 0 is always an exposed point of $B_{\mathcal{F}(M)}^{+}$, but $m_{x 0}=\delta(x) / d(x, 0)$ is exposed if and only if $[0, x]$ is trivial. Indeed, one implication is immediate from Theorem 5.3.3. The other one follows from the fact that $f \in B_{\operatorname{Lip}_{0}(M)}$ norms $m_{x 0}$ if and only if $f(x)=d(x, 0)$, but then $f(y)=d(y, 0)$ for any $y \in[0, x]$ so $f$ norms $m_{y 0}$ too. 


\section{Extreme points which are almost positive}

Finally, we will extend the conclusion of Corollary 5.2.7 to those elements of $B_{\mathcal{F}(M)}$ that may be expressed as a finitely supported perturbation of a positive element of $\mathcal{F}(M)$. We shall prove that these extreme points must have finite support and hence be elementary molecules.

Let $S$ be a non-empty subset of $M$. We can then define a generalization of the infconvolution given by (2.1) as follows. For $\alpha>0$ and $f \in \operatorname{Lip}(S)$ with $\|f\|_{L} \leq \alpha$, let $E_{\alpha}^{+} f: M \rightarrow \mathbb{R}$ be the function defined by

$$
\left(E_{\alpha}^{+} f\right)(x)=\inf \{f(q)+\alpha \cdot d(x, q): q \in S\}
$$

for $x \in M$. Notice that we have just replaced $\|f\|_{L}$ in (2.1) by the constant $\alpha$. It is immediate then from Proposition 2.1.9 that $E_{\alpha}^{+} f$ is the largest $\alpha$-Lipschitz extension of $f$ to $M$. That is, unlike the inf-convolution $E^{+} f$, this extension does not preserve the Lipschitz constant of $f$; instead, the Lipschitz constant may increase up to a value of $\alpha$. We will only be concerned with the particular case $\alpha=1$.

We require the following simple observation, which amounts to the fact that finding the maximum of a given function can be accomplished in a stratified way, i.e. finding maxima subjected to certain constraints and then looking for the maximum among all possible sets of constraints.

Lemma 5.3.5. Let $\mu, \lambda \in \mathcal{F}(M)$ be such that $\lambda \geq 0$. Let $S=\operatorname{supp}(\mu) \cup\{0\}$ and define

$$
N(f)=\left\langle\mu+\lambda, E_{1}^{+} f\right\rangle
$$

for $f \in B_{\operatorname{Lip}_{0}(S)}$. Then $N$ is a concave function that attains its maximum on $B_{\operatorname{Lip}_{0}(S)}$, and

$$
\max _{f \in B_{\mathrm{Lip}_{0}(S)}} N(f)=\|\mu+\lambda\|
$$

Proof. It is obvious that $N(f) \leq\|\mu+\lambda\|$ for any $f \in B_{\operatorname{Lip}_{0}(S)}$. By the HahnBanach theorem, there is $g \in B_{\operatorname{Lip}_{0}(M)}$ such that $\|\mu+\lambda\|=\langle\mu+\lambda, g\rangle$. Let $f=$ $g \uparrow_{S}$, then $f \in B_{\operatorname{Lip}_{0}(S)}$ and $E_{1}^{+} f \geq g$, so $\left\langle\lambda, E_{1}^{+} f\right\rangle \geq\langle\lambda, g\rangle$. Moreover, $\left(E_{1}^{+} f\right) \uparrow_{S}=$ $g \uparrow_{S}$ and hence $\left\langle\mu, E_{1}^{+} f\right\rangle=\langle\mu, g\rangle$ by Proposition 3.3.9. It follows that

$$
N(f)=\left\langle\mu, E_{1}^{+} f\right\rangle+\left\langle\lambda, E_{1}^{+} f\right\rangle \geq\langle\mu, g\rangle+\langle\lambda, g\rangle=\|\mu+\lambda\| .
$$

To show that $N$ is concave, note that this is equivalent to the map $f \mapsto\left\langle\lambda, E_{1}^{+} f\right\rangle$ being concave, i.e. to

$$
\left\langle\lambda, E_{1}^{+}(c f+(1-c) g)\right\rangle \geq c\left\langle\lambda, E_{1}^{+} f\right\rangle+(1-c)\left\langle\lambda, E_{1}^{+} g\right\rangle
$$


for any $f, g \in B_{\operatorname{Lip}_{0}(S)}$ and $c \in(0,1)$. Since $\lambda \geq 0$, it suffices to show that

$$
E_{1}^{+}(c f+(1-c) g) \geq c E_{1}^{+} f+(1-c) E_{1}^{+} g
$$

pointwise, that is

$$
\begin{aligned}
\inf _{q \in S}(c f(q)+(1-c) g(q) & +d(x, q)) \geq \\
& c \cdot \inf _{q \in S}(f(q)+d(x, q))+(1-c) \cdot \inf _{q \in S}(g(q)+d(x, q))
\end{aligned}
$$

for every $x \in M$. But this is obvious.

Theorem 5.3.6. Let $\lambda, \mu \in \mathcal{F}(M)$ be such that $\lambda \geq 0$ and $\mu$ has finite support. If $\lambda+\mu$ is an extreme point of $B_{\mathcal{F}(M)}$, then it has finite support.

Proof. Let $S=\operatorname{supp}(\mu) \cup\{0\}$, and consider the function $N: \mathcal{F}(M)^{+} \times B_{\operatorname{Lip}_{0}(S)} \rightarrow \mathbb{R}$ given by $N(\lambda, f)=\left\langle\lambda+\mu, E_{1}^{+} f\right\rangle$. Denote also $N_{\lambda}(f)=N(\lambda, f)$. By Lemma 5.3.5, $\|\lambda+\mu\|$ is the maximum of $N_{\lambda}(f)$ for $f \in B_{\operatorname{Lip}_{0}(S)}$, and $N_{\lambda}$ is a concave function for fixed $\lambda$. Moreover, it is easy to verify directly that $N_{\lambda}$ is continuous using the boundedness of $S$. It follows from concavity that $N_{\lambda}(f)=\|\lambda+\mu\|$ if and only if $f$ is a local maximum of $N_{\lambda}$, i.e. if and only if

$$
\left\langle\lambda, E_{1}^{+}(f+g)-E_{1}^{+} f\right\rangle \leq\left\langle\mu, E_{1}^{+} f-E_{1}^{+}(f+g)\right\rangle=\langle-\mu, g\rangle
$$

for all $g \in \operatorname{Lip}_{0}(S)$ in a neighborhood of 0 such that $f+g \in B_{\operatorname{Lip}_{0}(S)}$.

Suppose now that $\lambda$ has infinite support, and let $f \in B_{\operatorname{Lip}_{0}(S)}$ be such that $\|\lambda+\mu\|=N_{\lambda}(f)$. We will show that there is a nonzero $v \in \mathcal{F}(M)$ such that $\lambda \pm v \geq 0,\left\langle v, E_{1}^{+} f\right\rangle=0$, and $\left\langle v, E_{1}^{+}(f+g)-E_{1}^{+} f\right\rangle=0$ for all $g \in \operatorname{Lip}_{0}(S)$ in a neighborhood of 0 . The argument above will then imply that

$$
\|\lambda \pm v+\mu\|=N(\lambda \pm v, f)=N(\lambda, f) \pm\left\langle v, E_{1}^{+} f\right\rangle=N(\lambda, f)=\|\lambda+\mu\|
$$

so $\lambda+\mu$ cannot be an extreme point of $B_{\mathcal{F}(M)}$. Thus if $\lambda+\mu$ is extreme then $\lambda$, and therefore also $\lambda+\mu$, must be finitely supported.

For every non-empty subset $K \subset S$, define the set

$$
A_{K}=\left\{x \in M:\left(E_{1}^{+} f\right)(x)=f(q)+d(x, q) \text { if and only if } q \in K\right\} .
$$

That is, $A_{K}$ contains those points $x \in M$ where the infimum in the definition of $\left(E_{1}^{+} f\right)(x)$ is attained exactly for all $q \in K$ and nowhere else. Since $S$ is finite, the sets $A_{K}$ form a finite partition of $M$. Choose $K$ of the smallest possible cardinality such that $\operatorname{supp}(\lambda) \cap A_{K}$ contains at least three different points $p_{1}, p_{2}, p_{3} \neq 0$. Let

$$
\varepsilon=\frac{1}{4} \min \left\{\left(f\left(q^{\prime}\right)+d\left(p_{i}, q^{\prime}\right)\right)-\left(f(q)+d\left(p_{i}, q\right)\right): q \in K, q^{\prime} \in S \backslash K, i=1,2,3\right\}
$$


and choose $r \in(0, \varepsilon)$ such that the balls $B\left(p_{i}, r\right)$ are disjoint, do not contain the base point, and do not intersect the finite sets $\operatorname{supp}(\lambda) \cap A_{L}$ for any $L \subsetneq K$. By Proposition 3.3.10, for $i=1,2,3$ there exist non-negative functions $h_{i} \in \operatorname{Lip}_{0}(M)$ supported on $B\left(p_{i}, r\right)$ such that $\left\langle\lambda, h_{i}\right\rangle>0$. Now choose real constants $c_{1}, c_{2}, c_{3}$, not all of them equal to zero, such that

$$
\begin{array}{ll}
c_{1}\left\langle\lambda, h_{1}\right\rangle+c_{2}\left\langle\lambda, h_{2}\right\rangle+c_{3}\left\langle\lambda, h_{3}\right\rangle & =0 \\
c_{1}\left\langle\lambda, h_{1} \cdot E_{1}^{+} f\right\rangle+c_{2}\left\langle\lambda, h_{2} \cdot E_{1}^{+} f\right\rangle+c_{3}\left\langle\lambda, h_{3} \cdot E_{1}^{+} f\right\rangle & =0
\end{array}
$$

and $\left|c_{i}\right| \leq 1 /\left\|h_{i}\right\|_{\infty}$. Let $h=c_{1} h_{1}+c_{2} h_{2}+c_{3} h_{3}$ and $v=\lambda \circ W_{h}$.

Let us check that $v$ satisfies the required conditions. By construction, we have $\langle\lambda, h\rangle=0$ and $\left\langle v, E_{1}^{+} f\right\rangle=\left\langle\lambda, h \cdot E_{1}^{+} f\right\rangle=0$. Also,

$$
\langle\lambda \pm v, g\rangle=\left\langle\lambda, g \pm W_{h}(g)\right\rangle=\langle\lambda, g \cdot(1 \pm h)\rangle
$$

for any $g \in \operatorname{Lip}_{0}(M)$. By the choice of $c_{i}$ we have $1 \pm h \geq 0$ and so $\langle\lambda \pm v, g\rangle \geq 0$ whenever $g \geq 0$, that is, $\lambda \pm v \geq 0$. Also, choose $i \in\{1,2,3\}$ such that $c_{i} \neq 0$, then there is $\varphi \in \operatorname{Lip}_{0}(M)$ such that $\varphi=1$ on $B\left(p_{i}, r\right)$ and $\varphi=0$ on $\operatorname{supp}(h) \backslash B\left(p_{i}, r\right)$, hence $\langle v, \varphi\rangle=c_{i}\left\langle\lambda, h_{i}\right\rangle \neq 0$. This shows that $v \neq 0$.

Finally, let $x \in \operatorname{supp}(v)$. Then $x \in \operatorname{supp}(\lambda) \cap \operatorname{supp}(h)$ by (3.13), so there is $i \in\{1,2,3\}$ such that $x \in B\left(p_{i}, r\right)$. Therefore, if $q \in K, q^{\prime} \in S \backslash K$ then

$$
\begin{aligned}
f\left(q^{\prime}\right)+d\left(x, q^{\prime}\right) & \geq f\left(q^{\prime}\right)+d\left(p_{i}, q^{\prime}\right)-d\left(x, p_{i}\right) \\
& \geq f(q)+d\left(p_{i}, q\right)+4 \varepsilon-d\left(x, p_{i}\right) \\
& \geq f(q)+d(x, q)+4 \varepsilon-2 d\left(x, p_{i}\right) \\
& \geq f(q)+d(x, q)+2 \varepsilon
\end{aligned}
$$

and so $x \in A_{L}$ for some $L \subset K$, hence $x \in A_{K}$ by construction. If we now take any $g \in \operatorname{Lip}_{0}(S)$ such that $\|g\|_{\infty}<\varepsilon$ and $\|f+g\|_{L} \leq 1$, then

$$
f\left(q^{\prime}\right)+g\left(q^{\prime}\right)+d\left(x, q^{\prime}\right)>f(q)+g(q)+d(x, q)
$$

for any $q \in K, q^{\prime} \in S \backslash K$, and it follows that $\left(E_{1}^{+}(f+g)\right)(x)=\left(E_{1}^{+} f\right)(x)+\gamma$ where $\gamma=\min _{q \in K} g(q)$. Thus we get

$$
\left\langle v, E_{1}^{+}(f+g)-E_{1}^{+} f\right\rangle=\langle\lambda, h \cdot \gamma\rangle=\gamma \cdot\langle\lambda, h\rangle=0 .
$$

This completes the proof. 


\subsection{Open problems}

The most important open question about the extremal structure of $\mathcal{F}(M)$ is precisely the main one motivating the present work. Unfortunately we have not been able to solve it during the course of this thesis.

Problem 5.1. Are all extreme points of $B_{\mathcal{F}(M)}$ elementary molecules?

No counterexample is known at the time of writing, but it follows from the results presented here that any such counterexample must necessarily have infinite support (Corollary 5.2.7) and cannot be expressed as a positive functional plus a finite sum of evaluation functionals (Theorem 5.3.6). By passing from $\mathcal{F}(M)$ to the Lipschitz-free space over the support, we may assume that the support of any candidate counterexample is the whole metric space.

Let us also mention that, due to Theorem 5.3.3, Problem 5.1 can be equivalently split into the following pair of subproblems:

Problem 5.1a. Are all extreme points of $B_{\mathcal{F}(M)}$ exposed?

Problem 5.1b. Are all exposed points of $B_{\mathcal{F}(M)}$ elementary molecules?

Indeed, the answer to Problem 5.1 is positive if and only if the answer to the two smaller problems also is. They are probably easier to answer individually, as one does not just have to consider the exposed points but also the associated exposing functionals, which in this case are functions in $\operatorname{Lip}_{0}(M)$.

We will now briefly summarize the current knowledge about those metric spaces $M$ where Problem 5.1 is known to have a positive answer. For the most important case we need to introduce a few definitions. Let us raise a warning here that there are conflicting naming and notation standards for the following concepts:

\section{Definition 5.4.1.}

(a) A function $f: M \rightarrow \mathbb{R}$ is said to be locally flat at $p \in M$ if for every $\varepsilon>0$ there exists $\delta>0$ such that $x \neq y \in B(p, \delta)$ implies that

$$
\frac{|f(x)-f(y)|}{d(x, y)} \leq \varepsilon .
$$

If $f$ is locally flat at every point of $p$, then we just say that $f$ is locally flat. If moreover the value of $\delta$ depends only on $\varepsilon$ but not on $p$, then $f$ is uniformly locally flat.

(b) A function $f: M \rightarrow \mathbb{R}$ is said to be flat at infinity if for every $\varepsilon>0$ there exists a compact set $K \subset M$ such that $x \neq y \in M \backslash K$ implies that

$$
\frac{|f(x)-f(y)|}{d(x, y)} \leq \varepsilon .
$$


(c) The little Lipschitz space $\operatorname{lip}_{0}(M)$ is the space of all functions $f \in \operatorname{Lip}_{0}(M)$ that are uniformly locally flat and flat at infinity.

The space $\operatorname{lip}_{0}(M)$ is most often considered in the compact and Heine-Borel cases. In those cases it is easy to see that if $f$ is locally flat and flat at infinity then it is uniformly locally flat, and if $M$ is compact then every $f \in \operatorname{Lip}_{0}(M)$ is obviously flat at infinity.

Definition 5.4.2. Let $Y$ be a subspace of $\operatorname{Lip}_{0}(M)$. We say that $Y$ separates points of $M$ uniformly if there exists a constant $C<\infty$ such that, given any pair of different points $x, y$ in $M$, there is $f \in Y$ with $\|f\|_{L} \leq C$ and $f(x)-f(y)=d(x, y)$.

We may now state our desired sufficient condition for Problem 5.1:

Theorem 5.4.3 ([53, Theorem 4.38 and Corollary $\left.\left.4.41^{4}\right]\right)$. If $M$ is a Heine-Borel space and $\operatorname{lip}_{0}(M)$ separates points of $M$ uniformly, then $\mathcal{F}(M) \cong \operatorname{lip}_{0}(M)^{*}$ and every extreme point of $B_{\mathcal{F}(M)}$ is an elementary molecule.

Some known cases where the hypothesis of Theorem 5.4.3 holds, and therefore Problem 5.1 has a positive solution, are Heine-Borel spaces that are either countable [17] or Hölder [53, Proposition 4.31]. Other weaker cases that are enough to answer Problem 5.1 arise when $M$ is not Heine-Borel but $\mathcal{F}(M)$ has a predual that is a subspace of $\operatorname{lip}_{0}(M)$ with certain additional properties (see [23] for the exact definitions and results).

A completely unrelated sufficient condition was proved recently: Problem 5.1 has a positive solution when $M$ is a subset of an $\mathbb{R}$-tree, since every extreme point of $B_{\mathcal{F}(M)}$ is preserved in that case (see [7] for the definition of $\mathbb{R}$-tree and the proof of that result). This includes all closed subsets of $\mathbb{R}$ and all ultrametric spaces.

Since the solution to Problem 5.1 might be hard to obtain, it would be equally worthwhile to pursue partial answers for important but fairly general cases: when $M$ is compact, when $\mathcal{F}(M)$ is a dual space, or when $M$ is uniformly discrete. The solution is not even known when $M$ is assumed to be compact and $\mathcal{F}(M)$ is assumed to be a dual space. Indeed, if $\operatorname{lip}_{0}(M)$ is a predual of $\mathcal{F}(M)$ then the answer is positive by Theorem 5.4.3, but it is currently unknown whether it is possible for $\mathcal{F}(M)$ to be dual without $\operatorname{lip}_{0}(M)$ separating points of $M$ uniformly. ${ }^{5}$

\footnotetext{
${ }^{4}$ Here we feel obliged to mention that the proof of Corollary 4.41 given in [53] is not correct, as it merely shows that, for every $m \in \operatorname{ext} B_{\mathcal{F}(M)}$, there is $\zeta \in \beta \widetilde{M}$ such that $\langle m, f\rangle=\left\langle f, \Phi^{*} \delta_{\zeta}\right\rangle$ for any $f \in \operatorname{lip}_{0}(M)$ instead of any $f \in \operatorname{Lip}_{0}$. This does not imply that $m=\Phi^{*} \delta_{\zeta}$ as is claimed to complete the proof. However, it is possible to complete the argument by applying Proposition 4.21 therein and carrying out a case-by-case analysis. The proof of the weaker version found in the first edition of the book is correct [52, Corollary 3.3.6].

${ }^{5}$ This question has been solved, in a joint work by the author and Chris Gartland, soon after finishing the initial manuscript of this dissertation.
} 
As a final problem we may propose the following:

Problem 5.2. Characterize the metric spaces $M$ such that every extreme point of $B_{\mathcal{F}(M)}$ is preserved.

The results in [7] show that this is true whenever $M$ is a subset of an $\mathbb{R}$-tree. A positive solution to Problem 5.1 would also imply that any compact or Heine-Borel space satisfies this, thanks to Corollary 5.2.10. 



\section{Bibliography}

[1] R. J. Aliaga and A. J. Guirao, "On the preserved extremal structure of Lipschitz-free spaces," Studia Math., vol. 245, pp. 1-14, 2019. Available at https://doi.org/10.4064/sm170529-30-11 (cited on pp. 5, 105, 107, and 108)

[2] R. J. Aliaga, C. Noûs, C. Petitjean, and A. Procházka, "Compact reduction in Lipschitz free spaces," arXiv preprint, 2020. Available at https://arxiv.org/abs/ 2004.14250 (cited on pp. 5 and 70)

[3] R. J. Aliaga and E. Pernecká, "Integral representation and supports of functionals on Lipschitz spaces," arXiv preprint, 2020. Available at https: //arxiv.org/abs/2009.07663 (cited on pp. 5 and 74)

[4] R. J. Aliaga and E. Pernecká, "Normal functionals on Lipschitz spaces are weak* continuous," arXiv preprint, 2020. Available at https://arxiv.org/abs/2004.14310 (cited on p. 5)

[5] R. J. Aliaga and E. Pernecká, "Supports and extreme points in Lipschitz-free spaces," Rev. Mat. Iberoam., vol. 36, pp. 2073-2089, 2020. Available at https://doi.org/10.4171/rmi/1191 (cited on pp. 5, 29, 105, 107, and 108)

[6] R. J. Aliaga, E. Pernecká, C. Petitjean, and A. Procházka, "Supports in Lipschitz-free spaces and applications to extremal structure," J. Math. Anal. Appl., vol. 489, 124128, 2020. Available at https://doi.org/10.1016/j.jmaa.2020.124128 (cited on pp. 5, 29, and 105)

[7] R. J. Aliaga, C. Petitjean, and A. Procházka, "Embeddings of Lipschitz-free spaces into $\ell_{1}, "$ arXiv preprint, 2019. Available at https://arxiv.org/abs/1909.05285 (cited on pp. 5,128 , and 129)

[8] R. J. Aliaga and A. Rueda Zoca, "Points of differentiability of the norm in Lipschitz-free spaces," J. Math. Anal. Appl., vol. 489, 124171, 2020. Available at https://doi.org/10.1016/j.jmaa.2020.124171 (cited on pp. 5 and 22)

[9] L. Ambrosio and D. Puglisi, "Linear extension operators between spaces of Lipschitz maps and optimal transport," J. Reine Angew. Math., vol. 764, pp. 1-21, 2020. Available at https://doi.org/10.1515/crelle-2018-0037 (cited on pp. 73, 77, 79 , and 93)

[10] R. F. Arens and J. Eells, "On embedding uniform and topological spaces," Pacific J. Math., vol. 6, pp. 397-403, 1956. Available at https://projecteuclid.org/euclid. pjm/1103043959 (cited on p. 2) 
[11] J. Becerra Guerrero, G. López-Pérez, and A. Rueda Zoca, "Octahedrality in Lipschitz-free Banach spaces," Proc. Roy. Soc. Edinburgh Sect. A, vol. 148, pp. 447-460, 2018. Available at https://doi.org/10.1017/S0308210517000373 (cited on p. 27)

[12] V. I. Bogachev, Measure theory. Berlin-Heidelberg: Springer-Verlag, 2007. (cited on pp. 74 and 75 )

[13] B. Cascales, R. Chiclana, L. C. García-Lirola, M. Martín, and A. Rueda Zoca, "On strongly norm attaining Lipschitz maps," J. Funct. Anal., vol. 277, pp. 1677-1717, 2019. Available at https://doi.org/10.1016/j.jfa.2018.12.006 (cited on p. 35)

[14] Ş. Cobzaş, R. Miculescu, and A. Nicolae, Lipschitz functions, ser. Lecture Notes in Mathematics. Switzerland: Springer Nature, 2019, no. 2241. (cited on p. 15)

[15] M. Cúth and M. Johanis, "Isometric embedding of $\ell_{1}$ into Lipschitz-free spaces and $\ell_{\infty}$ into their duals," Proc. Amer. Math. Soc., vol. 145, pp. 3409-3421, 2017. Available at https://doi.org/10.1090/proc/13590 (cited on p. 25)

[16] M. Cúth, O. F. K. Kalenda, and P. Kaplický, "Finitely additive measures and complementability of Lipschitz-free spaces," Israel J. Math., vol. 230, pp. 409-442, 2019. Available at https://doi.org/10.1007/s11856-019-1829-y (cited on p. 72)

[17] A. Dalet, "Free spaces over some proper metric spaces," Mediterr. J. Math., vol. 12, pp. 973-986, 2015. Available at https://doi.org/10.1007/s00009-014-0455-5 (cited on p. 128)

[18] K. de Leeuw, "Banach spaces of Lipschitz functions," Studia Math., vol. 21, pp. 55-66, 1961. Available at https://doi.org/10.4064/sm-21-1-55-66 (cited on p. 2)

[19] J. Diestel and J. J. Uhl, Jr., Vector measures, ser. Mathematical Surveys. Providence, RI: American Mathematical Society, 1977, no. 15. (cited on p. 79)

[20] M. Dubei, E. D. Tymchatyn, and A. Zagorodnyuk, "Free Banach spaces and extension of Lipschitz maps," Topology, vol. 48, pp. 203-212, 2009. Available at https://doi.org/10.1016/j.top.2009.11.020 (cited on p. 27)

[21] R. Engelking, General topology, ser. Sigma Ser. Pure Math. Berlin: Heidermann Verlag, 1989, no. 6. (cited on p. 51)

[22] M. Fabian, P. Habala, P. Hájek, V. Montesinos, and V. Zizler, Banach space theory. The basis for linear and nonlinear analysis, ser. CMS Books in Mathematics. New York: Springer-Verlag, 2011. (cited on pp. 6 and 22)

[23] L. García-Lirola, C. Petitjean, A. Procházka, and A. Rueda Zoca, "Extremal structure and duality of Lipschitz free spaces," Mediterr. J. Math., vol. 15, Art. 69, 2018. Available at https://doi.org/10.1007/s00009-018-1113-0 (cited on pp. 3, 48, 107, and 128)

[24] L. García-Lirola, A. Procházka, and A. Rueda Zoca, "A characterisation of the Daugavet property in spaces of Lipschitz functions," J. Math. Anal. Appl., vol. 464, pp. 473-492, 2018. Available at https://doi.org/10.1016/j.jmaa.2018.04.017 (cited on pp. 3, 107, and 117)

[25] M. I. Garrido and J. A. Jaramillo, "Homomorphisms on function lattices," Monatsh. Math., vol. 141, pp. 127-146, 2004. Available at https://doi.org/10.1007/ s00605-002-0011-4 (cited on p. 50) 
[26] M. I. Garrido and A. S. Meroño, "The Samuel realcompactification of a metric space," J. Math. Anal. Appl., vol. 456, pp. 1013-1039, 2017. Available at https://doi.org/10.1016/j.jmaa.2017.07.033 (cited on pp. 50 and 51)

[27] C. Gartland, "Lipschitz free spaces over locally compact metric spaces," arXiv preprint, 2020. Available at https://arxiv.org/abs/2004.11951 (cited on p. 70)

[28] A. Godard, "Tree metrics and their Lipschitz-free spaces," Proc. Amer. Math. Soc., vol. 138, pp. 4311-4320, 2010. Available at https://doi.org/10.1090/ S0002-9939-2010-10421-5 (cited on p. 26)

[29] G. Godefroy, "Existence and uniqueness of isometric preduals: a survey," in Banach space theory (Iowa City, IA, 1987), ser. Contemporary Mathematics. Providence, RI: American Mathematical Society, 1989, no. 85, pp. 131-193. (cited on pp. 25 and 26)

[30] G. Godefroy and N. J. Kalton, "Lipschitz-free Banach spaces," Studia Math., vol. 159, pp. 121-141, 2003. Available at https://doi.org/10.4064/sm159-1-6 (cited on pp. 2 and 73 )

[31] G. Godefroy, G. Lancien, and V. Zizler, "The non-linear geometry of Banach spaces after Nigel Kalton," Rocky Mountain J. Math., vol. 44, pp. 1529-1583, 2014. Available at https://www.jstor.org/stable/26409387 (cited on p. 72)

[32] A. J. Guirao, V. Montesinos, and V. Zizler, "On preserved and unpreserved extreme points," in Descriptive topology and functional analysis, ser. Springer Proc. Math. Stat. Cham: Springer, 2014, no. 80, pp. 163-193. Available at https://doi.org/10.1007/978-3-319-05224-3_9 (cited on p. 105)

[33] P. Hájek and M. Novotný, "Some remarks on the structure of Lipschitz-free spaces," Bull. Belg. Math. Soc. Simon Stevin, vol. 24, pp. 283-304, 2017. Available at https://doi.org/10.36045/bbms/1503453711 (cited on p. 27)

[34] S. C. Hille and D. T. H. Worm, "Embedding of semigroups of Lipschitz maps into positive linear semigroups on ordered Banach spaces generated by measures," Integr. Equ. Oper. Theory, vol. 63, pp. 351-371, 2009. Available at https://doi.org/10.1007/s00020-008-1652-z (cited on pp. 73 and 77)

[35] Y. Ivakhno, V. Kadets, and D. Werner, "The Daugavet property for spaces of Lipschitz functions," Math. Scand., vol. 101, pp. 261-279, 2007. Available at https://doi.org/10.7146/math.scand.a-15044 (cited on pp. 117 and 121)

[36] Y. Ivakhno, V. Kadets, and D. Werner, "Corrigendum to: The Daugavet property for spaces of Lipschitz functions," Math. Scand., vol. 104, p. 319, 2009. Available at https://doi.org/10.7146/math.scand.a-15101 (cited on p. 121)

[37] J. A. Johnson, "Banach spaces of Lipschitz functions and vector-valued Lipschitz functions," Trans. Amer. Math. Soc., vol. 148, pp. 147-169, 1970. Available at https://doi.org/10.2307/1995044 (cited on p. 2)

[38] V. Kadets, "Lipschitz mappings of metric spaces," Izv. Vyssh. Uchebn. Zaved. Mat., vol. 83, pp. 30-34, 1985. Available at http://mi.mathnet.ru/eng/ivm7254 (cited on pp. 2 and 19)

[39] V. Kadets, M. Martín, and M. Soloviova, "Norm attaining Lipschitz functionals," Banach J. Math. Anal., vol. 10, pp. 621-637, 2016. Available at https://doi.org/10.1215/17358787-3639646 (cited on p. 35) 
[40] N. J. Kalton, "Spaces of Lipschitz and Hölder functions and their applications," Collect. Math., vol. 55, pp. 171-217, 2004. Available at https://www.raco.cat/ index.php/CollectaneaMathematica/article/view/56573 (cited on pp. 33 and 70)

[41] I. V. Kantorovich and G. S. Rubinstein, "On a functional space and certain extremum problems," Dokl. Akad. Nauk SSSR, vol. 115, pp. 1058-1061, 1957, in Russian. Available at http://mi.mathnet.ru/eng/dan22286 (cited on p. 2)

[42] J. Lawson, "Ordered probability spaces," J. Math. Anal. Appl., vol. 455, pp. 167-179, 2017. Available at https://doi.org/10.1016/j.jmaa.2017.05.046 (cited on p. 74)

[43] E. J. McShane, "Extension of range of functions," Bull. Amer. Math. Soc., vol. 40, pp. 837-842, 1934. Available at https://doi.org/10.1090/S0002-9904-1934-05978-0 (cited on p. 14)

[44] J. R. Munkres, Topology, 2nd ed. Pearson, 2013. (cited on p. 6)

[45] A. Naor and G. Schechtman, "Planar earthmover is not in $L_{1}$," SIAM J. Comput., vol. 37, pp. 804-826, 2007. Available at https://doi.org/10.1137/05064206X (cited on pp. 2 and 27 )

[46] V. G. Pestov, "Free Banach spaces and representations of topological groups," Funct. Anal. its Appl., vol. 20, pp. 70-72, 1986. Available at http://doi.org/10.1007/BF01077324 (cited on pp. 2 and 19)

[47] W. Rudin, Real and complex analysis, 3rd ed. New York: McGraw-Hill Book Co., 1987. (cited on pp. 6 and 74)

[48] S. Sakai, $C^{*}$-algebras and $W^{*}$-algebras, ser. Ergebnisse der Mathematik und ihrer Grenzgebiete. Berlin-Heidelberg-New York: Springer-Verlag, 1971, no. 60. (cited on p. 35)

[49] H. H. Schaefer, Banach lattices and positive operators, ser. Grundlehren der mathematischen Wissenschaften. New York-Heidelberg: Springer-Verlag, 1974, no. 215. (cited on p. 35)

[50] C. Villani, Optimal transport, old and new, ser. Grundlehren der mathematischen Wissenschaften. Berlin-Heidelberg: Springer-Verlag, 2009, no. 338. (cited on p. 2)

[51] N. Weaver, "Isometries of noncompact Lipschitz spaces," Canad. Math. Bull., vol. 38, pp. 242-249, 1995. Available at https://doi.org/10.4153/CMB-1995-035-3 (cited on pp. 34 and 106)

[52] N. Weaver, Lipschitz algebras, 1st ed. River Edge, NJ: World Scientific Publishing Co., 1999. (cited on pp. 2, 19, 34, 40, 120, and 128)

[53] N. Weaver, Lipschitz algebras, 2nd ed. River Edge, NJ: World Scientific Publishing Co., 2018. (cited on pp. 6, 13, 21, 24, 25, 34, 40, 47, 49, 51, 52, 62, 63, 65, 66, 73, $82,102,106,120$, and 128)

[54] N. Weaver, "On the unique predual problem for Lipschitz spaces," Math. Proc. Camb. Philos. Soc., vol. 165, pp. 467-473, 2018. Available at https://doi.org/10.1017/S0305004117000597 (cited on pp. 17 and 34)

[55] R. G. Woods, "The minimum uniform compactification of a metric space," Fund. Math., vol. 147, pp. 39-59, 1995. Available at https://www.impan. $\mathrm{pl} / \mathrm{en} /$ publishing-house/journals-and-series/fundamenta-mathematicae/all/147/1/ 108794/the-minimum-uniform-compactification-of-a-metric-space (cited on p. 50) 


\section{List of symbols}

$\begin{array}{ll}\|f\|_{L} & \text { Lipschitz constant of } f, 9 \\ \operatorname{Lip}(M) & \text { Space of real-valued Lipschitz functions on } M, 10 \\ \operatorname{Lip}_{0}(M) & \text { Lipschitz space over } M, 12 \\ \rho & \text { Distance to the base point, } 13 \\ E^{+} f & \text { Inf-convolution of } f, 14 \\ E^{-} f & \text { Sup-convolution of } f, 14 \\ \delta(x) & \text { Evaluation functional on } x, 16 \\ \delta & \text { Canonical embedding of } M \text { into } \mathcal{F}(M), 16 \\ \mathcal{F}(M) & \text { Lipschitz-free space over } M, 17 \\ \operatorname{Lip}_{0}(M, X) & X \text {-valued Lipschitz space over } M, 19 \\ \mathcal{F}_{M}(K) & \text { Lipschitz-free subspace, } 20 \\ m_{x y} & \text { Elementary molecule on points } x, y, 21 \\ M_{\text {oll }}(M) & \text { Set of elementary molecules on } M, 21 \\ X^{+} & \text {Positive elements of } X, 22 \\ B_{X}^{+} & \text {Positive unit ball of } X, 22 \\ W_{h} & \text { Weighting operator associated to } h, 30 \\ \phi \circ W_{h} & \text { Weighted version of } \phi \in \mathcal{F}(M)^{* *}, 31 \\ H_{n} & \text { Weighting function } H_{n}, 32 \\ G_{n} & \text { Weighting function } G_{n}, 32 \\ \Lambda_{n} & \text { Weighting function } \Lambda_{n}, 32 \\ \Pi_{n} & \text { Weighting function } \Pi_{n}, 32 \\ \phi_{s} & \text { Sum of the Kalton decomposition of } \phi, 33 \\ \mathcal{I}_{M}(K) & \text { Ideal of functions that vanish at } K, 40 \\ \mathcal{H}(Y) & \text { Hull of } Y, 40 \\ \operatorname{supp}(m) & \text { Support of } m, 43 \\ \beta M & \text { Stone-Čech compactification of } M, 49 \\ M^{\mathcal{U}} & \text { Uniform compactification of } M, 50 \\ f^{\mathcal{U}} & \text { Continuous extension of } f \text { to } M^{\mathcal{U}}, 50 \\ \mathcal{A}^{\mathcal{U}} & \text { Closure of } A \text { in } M^{\mathcal{U}}, 50 \\ M^{\mathcal{R}} & \text { Lipschitz realcompactification of } M, 51 \\ \phi_{0} & \text { Part of } \phi \text { concentrated at the base point, } 56 \\ & \end{array}$


$\phi_{\infty}$

$\phi_{A}$

$\mathcal{S}(\phi)$

$\mathcal{J}_{M}(K)$

$\mu^{+}$

$\mu^{-}$

$|\mu|$

$\mu \uparrow_{A}$

$\mathcal{M}(X)$

$\mathcal{L} \mu$

$\delta_{x}$

$\mathcal{M}_{0}(X)$

$s(\zeta)$

$\|m\|_{1}$

$|\phi|$

$[p, q]$

$(p, q)_{x}$

$[p, q]_{\mathcal{U}}$

$\widetilde{M}$

$\Phi$

$\mathcal{D}_{p q}$

$\mathcal{E}_{p q}$

$\pi_{1}, \pi_{2}$

$f_{p q}$

$[p, q]_{\varepsilon}$

$E_{\alpha}^{+} f$

$\operatorname{lip}_{0}(M)$
Part of $\phi$ concentrated at infinity, 56

Part of $\phi$ that avoids infinity, 58

Extended support of $\phi, 59$

Ideal of functions that vanish at $K$ and are locally

flat at $K, 62$

Positive variation of measure $\mu, 74$

Negative variation of measure $\mu, 74$

Total variation of measure $\mu, 74$

Restriction of measure $\mu$ to $A, 75$

Space of Radon measures on $X, 75$

Functional induced by measure $\mu, 77$

Dirac measure on $x, 77$

Radon measures on $X$ that vanish at 0,77

Eventual separation of $\zeta$ from $M, 83$

Weight of a finitely supported functional $m, 85$

Variation functional of $\phi, 98$

Metric segment between $p$ and $q, 107$

Gromov product of $p$ and $q$ at $x, 107$

Extended segment between $p$ and $q, 108$

Pairs of different points of $M, 110$

de Leeuw transform, 110

Elements of $\widetilde{M}$ coupled to $(p, q), 110$

Face of $B_{\mathcal{F}(M)^{* *}}$ containing $m_{p q}, 110$

Projections from $\widetilde{M}$ onto $M, 115$

Ivakhno-Kadets-Werner exposing function, 121

Neighborhood of the segment $[p, q], 121$

Maximal $\alpha$-Lipschitz extension of $f, 124$

Little Lipschitz space over $M, 128$ 


\section{Index of terms}

compactification, 49

Stone-Čech, 49

uniform, 50

de Leeuw transform, 110

derivation, 51

diameter, 7

function

flat at infinity, 127

Lipschitz, 9

locally flat, 62,127

non-expansive, 9

positive, 23

uniformly locally flat, 127

functional

avoids 0,54

avoids infinity, 54

concentrated at 0,54

concentrated at infinity, 54

evaluation, 16

finitely supported, 21

induced by measure, 77

majorizable, 24

normal, 34

positive, 23

sequentially normal, 70

variation, 98

Gromov product, 107

Hahn decomposition, 75

hull, 40

ideal, 40

incremental quotient, 10 inf-convolution, 14

isometry, 9

Jordan decomposition, 74

Kalton decomposition, 33

Lipschitz constant, 9

Lipschitz realcompactification, 51

majorant, 24

minimal, 94

measure

almost Radon, 75

Borel, 74

concentrated on a set, 75

Dirac, 77

inner regular, 74

negative variation, 74

outer regular, 74

positive variation, 74

Radon, 74

regular, 74

total variation, 74

metric segment, 107

extended, 108

trivial, 108

molecule, 21

norming set, 42

ordered vector space, 22

partition of unity, 15

point

base, 6 
denting, 106

exposed, 105

extreme, 105

metrically aligned, 107

preserved extreme, 105

strongly exposed, 106

radius, 7

space

concave, 120

equilateral, 25

Hölder, 120

Heine-Borel, 51

Lipschitz, 12

Lipschitz-free, 17

Lipschitz-free sub-, 20

little Lipschitz, 128

pointed, 6

radially discrete, 100

radially uniformly discrete, 100

uniformly discrete, 50

sup-convolution, 14

support

extended, 59

of a measure, 74

of an element of $\mathcal{F}(M), 43$

theorem

extreme molecule, 117

intersection, 42

McShane, 14

normal functional, 36

Rademacher, 26

universal extension, 18

variation, 98

weighting operator, 30 